Publications de l'IHES,

Vol. 97 no.1 (2003), pp. 61-179.

\title{
MODULI SPACES OF ABELIAN DIFFERENTIALS: THE PRINCIPAL BOUNDARY, COUNTING PROBLEMS, AND THE SIEGEL-VEECH CONSTANTS
}

\author{
ALEX ESKIN, HOWARD MASUR, AND ANTON ZORICH
}

\begin{abstract}
A holomorphic 1-form on a compact Riemann surface $S$ naturally defines a flat metric on $S$ with cone-type singularities. We present the following surprising phenomenon: having found a geodesic segment (saddle connection) joining a pair of conical points one can find with a nonzero probability another saddle connection on $S$ having the same direction and the same length as the initial one. A similar phenomenon is valid for the families of parallel closed geodesics.

We give a complete description of all possible configurations of parallel saddle connections (and of families of parallel closed geodesics) which might be found on a generic flat surface $S$. We count the number of saddle connections of length less than $L$ on a generic flat surface $S$; we also count the number of admissible configurations of pairs (triples,...) of saddle connections; we count the analogous numbers of configurations of families of closed geodesics. By the previous result of EMa these numbers have quadratic asymptotics $c \cdot\left(\pi L^{2}\right)$. Here we explicitly compute the constant $c$ for a configuration of every type. The constant $c$ is found from a Siegel-Veech formula.

To perform this computation we elaborate the detailed description of the principal part of the boundary of the moduli space of holomorphic 1-forms and we find the numerical value of the normalized volume of the tubular neighborhood of the boundary. We use this for evaluation of integrals over the moduli space.
\end{abstract}

\section{Contents}

\section{Introduction}

1. Flat Surfaces and Geodesics on Flat Surfaces 3

1.1. Flat Surfaces and Abelian Differentials 6

2. Moduli Spaces of Abelian Differentials 6

2.1. Stratification 6

2.2. Local Coordinates and Volume Element

3. Principal Boundary of the Moduli Spaces and Method of Evaluation of the Siegel-Veech Constants

3.1. Holonomy and Homology

3.2. Configurations of Saddle Connections 10

3.3. Siegel-Veech Formula 12

3.4. Principal Boundary of the Moduli Spaces 13

3.5. Arithmetic of Siegel - Veech Constants 15

4. Readers Guide 15

Date: November 11, 2003.

Research of the first author is partially supported by NSF grant DMS-9704845, the Sloan Foundation and the Packard Foundation.

Research of the second author is partially supported by NSF grant 9803497 . 
Part 0. Structure of the Strata 16

5. Connected Components of the Strata 16

5.1. Parity of a Spin Structure 16

5.2. Classification of Connected Components of the Strata 16

6. Nonprimitive Strata 17

6.1. Strata of Surfaces with Marked Points 17

6.2. Strata of Disconnected Surfaces 18

7. Thick-Thin Decomposition, Volume Estimates, and Computation of the Siegel-Veech Constants

Part 1. Saddle Connections Joining Distinct Zeroes 22

8. Saddle Connections of Multiplicity One. Connected Strata 22

8.1. Breaking up a Zero 22

8.2. Collapsing a Pair of Zeroes, Principal Boundary 24

8.3. Computing the Siegel-Veech Constants 26

8.4. Examples: Constants for Connected Strata in Genus 3

8.5. Constants for the Principal Stratum $\mathcal{H}_{1}(1, \ldots, 1)$

9. Multiple Homologous Saddle Connections. Connected Strata 29

9.1. Principal Boundary 29

9.2. Slit Construction 30

9.3. Building Surfaces With Multiple Homologous Saddle Connections 30

9.4. Stratum Interchange and $\gamma \rightarrow-\gamma$ Symmetry 32

9.5. Siegel-Veech Constants in Higher Multiplicity, Connected Strata 35

9.6. Principal Stratum 39

10. Strata Having Several Connected Components 40

10.1. Parity of the Spin Structure in the Slit Construction 40

10.2. Admissible Assignments for Surfaces from Hyperelliptic Components 41

10.3. Constants for the Components of the Stratum $\mathcal{H}_{1}(g-1, g-1)$

10.4. Other Strata 47

Part 2. Saddle Connections Joining a Zero to Itself 49

11. Approaching the Principal Boundary by Shrinking Closed Geodesics 49

11.1. Configurations of Closed Saddle Connections and Corresponding Surface Decompositions

11.2. Shrinking a Pair of Adjacent Holes

11.3. Transporting a Small Hole Along a Flat Surface

11.4. Shrinking a Pair of Nonadjacent Holes 55

12. Constructing Surfaces with Homologous Closed Saddle Connections 55

12.1. Figure Eight Construction 55

12.2. Creating a Pair of Holes 56

12.3. Admissible Constructions 57

13. Computing the Siegel-Veech Constants for Connected Strata 61

13.1. Computation of the Constants 61

13.2. Stratum Interchange and $\gamma \rightarrow-\gamma$ Symmetry 66

13.3. Combinatorial Factor, Connected Strata 68

13.4. Examples: Constants for Strata in Genus 3

13.5. Principal Stratum 72

14. Strata that are not Connected 75 
14.1. Parity of the Spin Structure of the Compound Surface

14.2. Decomposition of Surfaces from Hyperelliptic Components

14.3. Constants for the Hyperelliptic Connected Components

14.4. Connected Component $\mathcal{H}^{\text {nonhyp }}(g-1, g-1)$; Even Genus $g \quad 82$

14.5. Nonhyperelliptic Components with a Spin Structure

Appendix. Values of the Constants

Appendix A. Saddle Connections Joining Distinct Zeroes $\quad 88$

A.1. Connected Strata in Genus 4

Appendix B. Saddle Connections Joining a Zero to Itself

B.1. Connected Strata in Genus 4

B.2. Closed Geodesics for Nonconnected Strata in Genus 4

References

\section{Introduction}

\section{Flat Surfaces and Geodesics on Flat Surfaces}

In this paper we shall consider flat metrics with isolated conical singularities on a closed orientable surface of genus $g$. An important class of such flat metrics corresponds to the translation surfaces: those surfaces for which the flat metric on the surface has trivial linear holonomy. These surfaces have been studied by several authors in various guises; in the context of Abelian differentials (and more generally quadratic differentials) on compact Riemann surface in $[\underline{\underline{S}}$, in the context of rational billiards in KaZe, KMS, Ve3, and [GJ]. Moduli spaces of these structures have been studied in Ve4, MS, Ko, KonZo, and EMa]. A survey is given in $[\mathrm{MT}]$.

Triviality of holonomy means, in particular, that the parallel transport of a vector along a small loop going around a conical point brings a vector back to itself. This implies that all cone angles of such surface are integer multiples of $2 \pi$.

Choose a direction in the tangent space to some base point $x_{0} \in S$ of the flat surface and transport this direction using the parallel transport to all nonsingular points of the surface. Since the monodromy is trivial this parallel transport does not depend on the path. Thus, any direction is globally defined on the flat surface punctured at the singularities. Throughout this paper by a flat surface we mean a flat surface having trivial holonomy representation in $S O(2, \mathbb{R})$. Following a tradition we often call the conical points the saddles.

A flat torus gives an example of a flat surface without any saddles. One can glue a flat torus from a parallelogram by identifying opposite sides. A generalization can be found by considering a polygon in the plane having the property that its sides are distributed into pairs that are parallel and of equal length. Identifying the pairs by translations one gets a closed oriented flat surface. For example, identifying the opposite sides of a regular octagon one gets a surface of genus 2, see Figure 1 All vertices of the octagon are identified to a single saddle which has an angle of $6 \pi$. Deforming the regular octagon in such way that the sides are organized into pairs of parallel sides of equal length we get a family of flat surfaces of genus $g=2$ each having a single conical singularity with the cone angle $6 \pi$. 



Figure 1. Identifying the opposite sides of these octagons by parallel translations we obtain a pair of flat surfaces of genus $g=2$.

We may assume that we have cartesian coordinates in the polygons. In these coordinates away from the saddles, geodesics on the surface $S$ are straight lines. The behavior of geodesics on flat surfaces is in many aspects similar to the behavior of geodesics on a flat torus. In particular, a geodesic cannot change the direction, and thus cannot have self intersections. A generic geodesic is dense in the surface analogously to an irrational winding line which is dense on the torus.

However, a flat surface different from the torus must have saddles and some geodesics that hit the saddles. Some geodesics hit the saddles going both in forward and in backward directions. Such a geodesic segment joining a pair of saddles and having no saddles in its interior is called a saddle connection.

Note that the shortest representative in a homotopy class of curves joining two saddles exists. Typically, even for the class of a simple closed curve, one gets a broken line containing many geodesic segments. The segments of these broken lines pass through the saddles changing directions there. We want to stress that throughout this paper we consider only those saddle connections which contain a single geodesic segment and thus no saddles in its interior. However, we consider the situation when the endpoints of a saddle connection coincide. We call such saddle connections the closed saddle connections.

A geodesic leaving a regular point $P$ may return back to $P$ without meeting any singularities. Since it cannot change its direction, we necessarily get a closed regular geodesic in this case. Note that as in the case of the torus, any geodesic leaving a nearby point in a parallel direction will be also closed and will have the same length as the initial one. Thus closed regular geodesics appear in families of parallel geodesics of the same length. However, unlike the case of the torus, such parallel closed geodesics do not typically fill all the surface, but only a cylindrical subset. Each boundary component of such a cylinder is comprised of saddle connections. For example, choosing the vertical direction on the flat surface obtained from a regular octagon (see Figure 1) we get two families of regular closed geodesics, and each of the two families fills a cylinder. Generically, each boundary component of a cylinder filled with closed geodesics is a single closed saddle connection.

The converse, however, is false. A closed saddle connection does not necessarily bound a cylinder of regular closed geodesics. In fact, it bounds such a cylinder if and only if the angle at the saddle between the outgoing and incoming segments is exactly $\pi$. In this paper we will be interested in both counting closed saddle connections and counting cylinders filled with regular closed geodesics.

Now fix a flat surface $S$. Let $\hat{S}$ be the universal cover of $S-\Sigma$, where $\Sigma$ is the collection of saddles. There is an isometric map of $\hat{S}$ to $\mathbb{R}^{2}$ called the developing 
map which maps a lift $\hat{\gamma}$ of an oriented curve $\gamma$ on $S$ to a curve in $\mathbb{R}^{2}$. We denote by $\operatorname{hol}(\gamma)$ the difference of the endpoints of the image. The vector $\operatorname{hol}(\gamma) \in \mathbb{R}^{2}$ is called the holonomy vector of $\gamma$.

Remark 1.1. By definition a flat surface has trivial holonomy representation in the linear group

$$
\pi_{1}(S) \rightarrow H_{1}(S, \mathbb{Z}) \rightarrow S O(2, \mathbb{R}) .
$$

However, the holonomy representation in the affine group is nontrivial: its image belongs to the group of translations. From now on we reserve the notion of holonomy for this second representation. It matches the notion of holonomy $\operatorname{hol}(\gamma)$ defined by an arbitrary (not necessarily closed) oriented curve $\gamma$ suggested in the paragraph above.

Now fix a pair of saddles on a flat surface $S$, and some length $L$. Consider all those saddle connections joining the fixed saddles whose length is shorter than $L$. We are interested in the asymptotics of the number of saddle connections when the bound $L$ tends to infinity. In the model case of the torus of unit area it is sufficient to pass to the universal covering plane to see that the number of geodesic segments of length at most $L$ joining a generic pair of distinct points on the torus grows quadratically as the number of lattice points in a disc of radius $L$, so we get asymptotics $\pi L^{2}$. Note that the number of (homotopy classes) of closed geodesics of length at most $L$ has different asymptotics. Since we want to count only primitive geodesics (those which do not repeat themselves) now we have to count only coprime lattice points in a disc of radius $L$, considered up to a symmetry of the torus (issues of symmetry will be considered in details later). Therefore we get the asymptotics $\frac{1}{2 \zeta(2)} \cdot \pi L^{2}=\frac{3}{\pi^{2}} \cdot \pi L^{2}$

It is proved in EMa that the growth rate of the number of saddle connections for a generic flat surface also has quadratic asymptotics $c \cdot\left(\pi L^{2}\right)$, and, moreover, almost all flat surfaces in some natural families of flat surfaces share the same constant $c$ in the asymptotics. One of the principal goals of this paper is to compute these constants for all natural families (which are explicitly defined below). We also compute the constants in the quadratic asymptotics for the number of families of closed regular geodesics.

It is relatively easy to show that for a generic flat surface one can never find a pair of saddle connections having the same direction but different lengths, see Proposition 7.4 at the end of Section[7 However, somewhat surprisingly, the configurations of pairs of parallel saddle connections of the same length and same direction can be found on almost every flat surface, and, what may seem even more surprising, their number also has quadratic asymptotics (with another constant $c$ ).

One can ask now the similar questions about the triples of saddle connections, or about some more specific configurations of saddle connections, or similar questions concerning closed geodesics. We give a complete description of those configurations which can be found on a generic surface; we show that each of them has quadratic asymptotics (this was essentially already proved in [EMa]), and in every case we compute the corresponding constant in the quadratic asymptotics.

Remark 1.2. A billiard in a polygon with rational angles gives rise to a flat surface, see for example KaZe, [KMS, or [MT]. However, rational billiards are not generic in the natural families of flat surfaces, which we consider. Thus, the constants 
described in this paper do not apply to the billiard problem, with an exception for some particular classes off billiards, see [EZ].

1.1. Flat Surfaces and Abelian Differentials. If we cut a flat surface $S$ successively along an appropriate collection of saddle connections we can decompose it into polygons contained in $\mathbb{R}^{2}=\mathbb{C}$, see, for example, Figure 1 We may then view $S$ as a union of polygons glued along parallel sides by means of parallel translations. Note that we have endowed every polygon with a complex coordinate. Since our gluing rules are just translations, the transition functions in these complex coordinates have the form

$$
z \rightarrow z+\text { const. }
$$

Thus any flat surface with the conical singularities removed is endowed with a natural complex structure. Moreover, consider a holomorphic 1-form $\omega=d z$ on every polygon. Since $d z=d(z+$ const $)$ we obtain a globally defined holomorphic 1 -form on the surface with removed singularities. It is an easy exercise to show that the complex structure and the holomorphic 1-form can be extended to the singularities; the holomorphic 1-form (or, what is the same, the Abelian differential) $\omega$ has a zero at every conical point.

Conversely, given a pair $(M, \omega)$ where $M$ is a Riemann surface, $\omega$ is a holomorphic Abelian differential on $M$, and a point $P \in M$ such that $\omega(P) \neq 0$, there exists a local coordinate $z$ near $P$ such that $\omega=d z$. Such a local coordinate is unique up to change of coordinates $z \rightarrow z+$ const. Thus $|d z|^{2}$ is a flat metric on $M$; this metric develops conical singularities at the zeroes of $\omega$. At a zero of order $k$ the total angle is $2 \pi(k+1)$. Such collection of coordinate charts determines a structure $S=(M, \omega)$ of a "translation surface" (which we agree to call just a "flat surface") on $M$, namely, an atlas of coordinate charts which cover the surface away from the singularities, such that the transition functions are translations

$$
z \rightarrow z+\text { const. }
$$

When we consider moduli spaces of flat surfaces we will wish to distinguish between $\omega$ and $e^{i \theta} \omega$. This is equivalent to specifying a distinguished direction on the flat surface.

Convention 1. Depending on the context we shall use one of the synonyms "saddle", "conical singularity", "zero of Abelian differential" or just "zero".

\section{Moduli Spaces of Abelian Differentials}

2.1. Stratification. Let $\alpha$ be a partition of $2 g-2$ (i.e. a representation of $2 g-2$ as an unordered sum of positive integers). Let $\mathcal{H}(\alpha)$ denote the moduli space of pairs $(M, \omega)$ where $M$ is a closed Riemann surface of genus $g$, and $\omega$ is a holomorphic Abelian differential on $M$ such that the orders of its zeroes is given by $\alpha$. Here we distinguish between $\omega$ and $e^{i \theta} \omega$. The zeroes on each surface are assumed to be named. We say that two such structures are equivalent if there is an isomorphism from one to the other which takes zeroes to zeroes, preserving the naming. The set of equivalence classes is denoted $\mathcal{H}(\alpha)$ and is called a stratum. This term is justified by the fact that the space of all Abelian differentials on Riemann surfaces of genus $g$ is stratified by the spaces $\mathcal{H}(\alpha)$, as $\alpha$ varies over the partitions of $2 g-2$. In the case of flat tori the space is $G L_{+}(2, \mathbb{R}) / S L(2, \mathbb{Z})$, the moduli space of lattices. The stratum corresponding to the partition $(1, \ldots, 1)$ is called the principal stratum: it 
corresponds to holomorphic differentials with simple zeroes. For example, the surface in Figure 2 belongs to $\mathcal{H}(1,1)$. The stratum might be nonconnected, although the components have been completely classified (see [KonZ0). The classification involves the notion of parity of spin structure and issues of hyperellipticity. These components will be described later in Section 5.2

2.2. Local Coordinates and Volume Element. The stratum $\mathcal{H}(\alpha)$ can be topologized and given a natural "Lebesgue" measure as follows. For a flat surface $S_{0} \in \mathcal{H}(\alpha)$ with conical points $P_{1}, \ldots, P_{k}$, choose a basis of cycles for the relative homology $H_{1}\left(S_{0},\left\{P_{1}, \ldots, P_{k}\right\} ; \mathbb{Z}\right)$. This basis may be chosen in such a way that each element is represented by a saddle connection. Equivalently, the saddle connections cut $S$ into a union of polygons. For any $S$ near $S_{0}$ the vectors associated to these saddle connections serve as local coordinates, see, for example, Figure 1. By construction these vectors are the relative periods of the Abelian differential $\omega$ (i.e. the integrals of $\omega$ along the paths joining points $P_{i}$ and $P_{j}$, where $i=j$ is also allowed). So we actually use a domain in the space of relative cohomology $H^{1}\left(S_{0},\left\{P_{1}, \ldots, P_{k}\right\} ; \mathbb{C}\right)$ as a local coordinate chart. Ignoring the complex structure we get a domain in $\mathbb{R}^{n}=H^{1}\left(S_{0},\left\{P_{1}, \ldots, P_{k}\right\} ; \mathbb{C}\right)$, where $n=4 g+2 k-2$. Note that we have a natural cubic lattice in our coordinates given by the "integer" cohomology

$$
H^{1}\left(S_{0},\left\{P_{1}, \ldots, P_{k}\right\} ; \mathbb{Z} \oplus i \mathbb{Z}\right) \subset H^{1}\left(S_{0},\left\{P_{1}, \ldots, P_{k}\right\} ; \mathbb{C}\right)=\mathbb{R}^{n}
$$

We define a measure or volume element $d \nu(S)$ on $\mathcal{H}(\alpha)$ as Lebesgue measure defined by these coordinates, normalized so that the volume of a unit cube in $\mathbb{R}^{n}$ is 1 . One easily checks that the volume element is well-defined: it is independent of choice of basis.

Let $\mathcal{H}_{1}(\alpha) \subset \mathcal{H}(\alpha)$ be the hypersurface in $\mathcal{H}(\alpha)$ of unit area flat surfaces. Choose a symplectic homology basis of closed curves $A_{i}, B_{i}, i=1, \ldots, g$ on the surface $S=(M, \omega)$. The area of the flat surface $S$ defined by the Abelian differential $\omega$ is given by the Riemann bilinear relation,

$$
\int_{S}|\omega|^{2} d x d y=\frac{i}{2} \int_{S} \omega \wedge \bar{\omega}=\frac{i}{2} \sum_{i}\left(\int_{A_{i}} \omega \int_{B_{i}} \bar{\omega}-\int_{A_{i}} \bar{\omega} \int_{B_{i}} \omega\right)
$$

Thus the unit area surfaces represent a "hyperboloid" in terms of the coordinates chosen above.

Throughout this paper we shall mostly work with the "hyperboloid" $\mathcal{H}_{1}(\alpha) \subset$ $\mathcal{H}(\alpha)$. The volume element in the embodying space $\mathcal{H}(\alpha)$ induces naturally a volume element on the hypersurface $\mathcal{H}_{1}(\alpha)$ in the following way. There is a natural $\mathbb{C}^{*}$-action on $\mathcal{H}(\alpha)$ : having $\lambda \in \mathbb{C}-\{0\}$ we associate to the flat surface $S=(M, \omega)$ the flat surface $\lambda \cdot S=(M, \lambda \cdot \omega)$. In particular, we can represent any $S \in \mathcal{H}(\alpha)$ as $S=r S^{\prime}$, where $r \in \mathbb{R}_{+}$, and where $S^{\prime}$ belongs to the "hyperboloid": $S^{\prime} \in$ $\mathcal{H}_{1}(\alpha)$. Geometrically this means that the metric on $S$ is obtained from the metric on $S^{\prime}$ by rescaling with coefficient $r$. In particular, vectors associated to saddle connections on $S^{\prime}$ are multiplied by $r$ to give vectors associated to corresponding saddle connections on $S$. It means also that area $(S)=r^{2} \cdot \operatorname{area}\left(S^{\prime}\right)=r^{2}$, since area $\left(S^{\prime}\right)=1$. We define the volume element $d \operatorname{vol}\left(S^{\prime}\right)$ on the "hyperboloid" $\mathcal{H}_{1}(\alpha)$ by disintegration of the volume element $d \nu(S)$ on $\mathcal{H}(\alpha)$ :

$$
d \nu(S)=r^{n-1} d r d \operatorname{vol}\left(S^{\prime}\right),
$$


where $n=\operatorname{dim}_{\mathbb{R}} \mathcal{H}(\alpha)$. Using this volume element we define the total volume of the stratum $\mathcal{H}_{1}(\alpha)$ :

$$
\operatorname{Vol}\left(\mathcal{H}_{1}(\alpha)\right):=\int_{\mathcal{H}_{1}(\alpha)} d \operatorname{vol}\left(S^{\prime}\right) .
$$

Since the measure on $\mathcal{H}_{1}(\alpha)$ is induced by the volume element, the total volume of the stratum coincides with the total measure.

For a subset $E \subset \mathcal{H}_{1}(\alpha)$ we let $C(E) \subset \mathcal{H}(\alpha)$ denote the "cone" based on $E$ :

$$
C(E):=\left\{S=r S^{\prime} \mid S^{\prime} \in E, 0<r \leq 1\right\} .
$$

Our definition of the volume element on $\mathcal{H}_{1}(\alpha)$ is consistent with the following normalization:

$$
\operatorname{Vol}\left(\mathcal{H}_{1}(\alpha)\right)=n \cdot \nu\left(C\left(\mathcal{H}_{1}(\alpha)\right),\right.
$$

where $n=\operatorname{dim}_{\mathbb{R}} \mathcal{H}(\alpha)$, and $\nu\left(C\left(\mathcal{H}_{1}(\alpha)\right)\right.$ is the total volume (total measure) of the "cone" $C\left(\mathcal{H}_{1}(\alpha)\right) \subset \mathcal{H}(\alpha)$ measured by means of the volume element $d \nu(S)$ on $\mathcal{H}(\alpha)$ defined above.

Convention 2. We need to use both volume elements. To distinguish them we keep the notation $d \nu(S)$ for the volume element in the whole stratum $\mathcal{H}(\alpha)$ and notation $d \operatorname{vol}(S)$ for the volume (hypersurface) element in the "unit hyperboloid" $\mathcal{H}_{1}(\alpha)$ which is a hypersurface in $\mathcal{H}(\alpha)$.

It is a result of $[\mathrm{M}], \mathrm{MS}]$ and $\mathrm{Ve} 2$ that these volumes are finite. Their values have been calculated in $\mathrm{EOk}$, see also $\mathrm{ZO}$ for the volumes of some low-dimensional strata. They will be needed for the calculations of the asymptotic constants. Tables of the values of the volumes for all connected components of the strata in genera $g \leq 4$ are presented in section 8.4

\section{Principal Boundary of the Moduli Spaces and Method of Evaluation of the Siegel-Veech Constants}

3.1. Holonomy and Homology. Note that in terms of the local coordinates $(x, y)$ defining the flat structure the corresponding Abelian differential has the form $\omega=$ $d z=d x+i d y$. This has an important consequence: for any oriented curve $\beta$ on $S$, the holonomy of $\beta$ (as defined above in terms of the developing map) coincides with the integral $\int_{\beta} \omega$ of $\omega$ over $\beta$ (here we have identified $\mathbb{R}^{2}$ and $\mathbb{C}$ ). Since the 1 -form $\omega$ is closed, it means, in particular, that if $\beta$ and $\gamma$ are homologous, then $h o l(\gamma)=h o l(\beta)$. In particular, if we have a cylinder filled with regular closed geodesics, then all these geodesics share the same holonomy vector.

When a path $\beta$ joins a pair of distinct points we say that a path $\gamma$ is homologous to $\beta$ if it joins the same pair of points and if the closed loop $\beta \cdot \gamma^{-1}$ is homologous to zero, that is breaks the surface into two components. The paths $\beta, \gamma$ which interest us in this paper are saddle connections or closed geodesics on $S$. We can formalize the above observation saying that saddle connections $\beta, \gamma$ representing homologous elements in $H_{1}\left(S,\left\{P_{1}, \ldots, P_{k}\right\} ; \mathbb{Z}\right)$ (where $P_{1}, \ldots, P_{k}$ are the saddles) share the same holonomy vector $\operatorname{hol}(\beta)=\operatorname{hol}(\gamma)$.

On the other hand the equality $\operatorname{hol}(\beta)=\operatorname{hol}(\gamma)$ for nonhomologous $\beta, \gamma$ occurs only for non generic surfaces. Consider the following example. Take four unit squares with sides glued as indicated at the left-hand-side picture at Figure 2 The result is a closed surface $S$ of genus 2 . The five points indicated by the filled circle 
symbol are identified, as are the five points indicated by the unfilled circle symbol. Hence $S$ has two conical singularities, each of total angle $4 \pi$. Each of the hatched squares corresponds to a cylinder filled with horizontal regular closed geodesics of length 1 . Hence, for any closed geodesics $\sigma_{1}$ in the first cylinder and any closed geodesics $\sigma_{2}$ in the second cylinder we get $h o l\left(\sigma_{1}\right)=h o l\left(\sigma_{2}\right)=(1,0)$.

Note, however, that $\sigma_{1}$ and $\sigma_{2}$ are not homologous. This means that the relation above does not survive under a generic deformation of this flat surface, see the righthand-side picture at Figure 22 On the other hand the pair of saddle connections $\gamma$ and $\gamma_{1}$ are homologous and we see that they keep sharing the same holonomy $\operatorname{hol}(\gamma)=\operatorname{hol}\left(\gamma_{1}\right)$ under any small deformation of the surface. Figure 111 gives an example when such a relation between two distinct families of closed geodesics is stable under any small deformation of the flat surface.
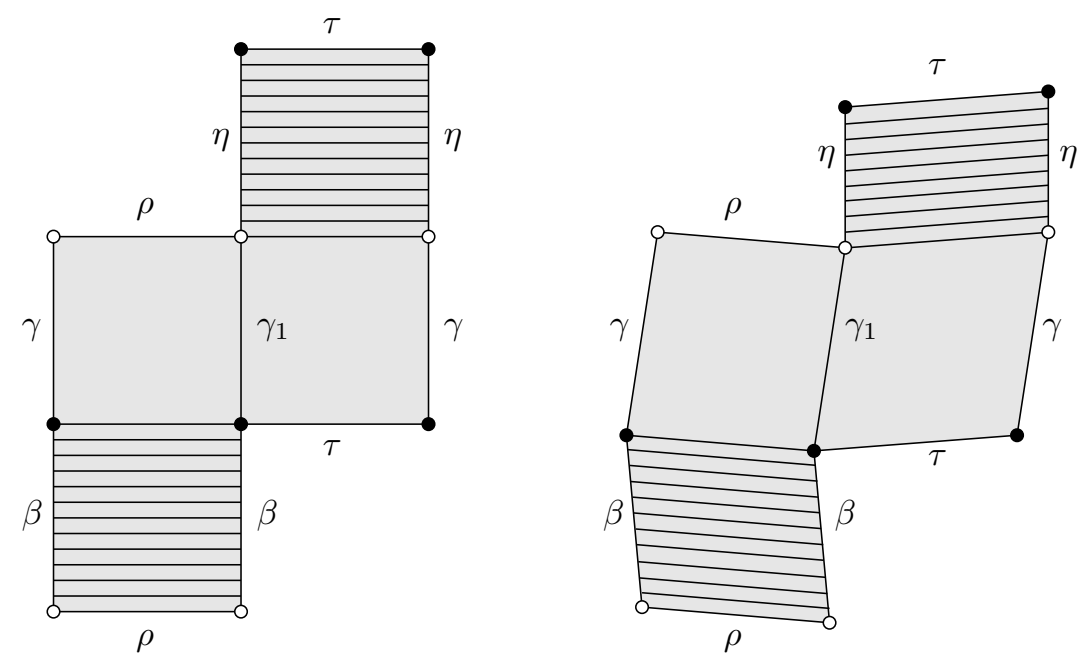

Figure 2. Nonhomologous saddle connections which have the same holonomy lose this property after a generic deformation of the surface, while homologous ones, $\gamma \sim \gamma_{1}$, keep the same holonomy.

This observation can be formalized as follows.

Proposition 3.1. Almost any flat surface $S$ in any connected component of any stratum does not have a single pair of nonhomologous saddle connections sharing the same holonomy vector.

Proof. Consider a basis of (relative) cycles in $H_{1}\left(S,\left\{P_{1}, \ldots, P_{k}\right\} ; \mathbb{Z}\right)$. We know that the corresponding periods of $\omega$ (integrals over these basic cycles) serve as local coordinates in the stratum $\mathcal{H}(\alpha)$. Note that for almost all points of the cohomology space these periods are rationally independent: no linear combination of periods equals to zero when the linear combination is taken with rational coefficients. Note also, that if the periods of a closed 1-form $\omega$ are rationally independent, then the same property is obviously true for the form $\lambda \cdot \omega$ where $\lambda \in \mathbb{R}$. Hence, this is true for almost all flat surfaces in the "unit hyperboloid" $\mathcal{H}_{1}(\alpha)$ 
A pair of saddle connections $\beta, \gamma$ represent integer cycles in $H_{1}\left(S,\left\{P_{1}, \ldots, P_{k}\right\} ; \mathbb{Z}\right)$. Hence, if they are not homologous, for almost all points $(S, \omega)$ of $\mathcal{H}_{1}(\alpha)$ the corresponding periods are different: $\int_{\beta} \omega \neq \int_{\gamma} \omega$. Since $h o l(\beta)=\int_{\beta} \omega$ and $\operatorname{hol}(\gamma)=\int_{\gamma} \omega$ the Proposition is proved.

3.2. Configurations of Saddle Connections. Consider the set of all saddle connections on a flat surface $S$ and consider its image $V_{s c}(S) \subset \mathbb{R}^{2}$ under the holonomy map. (We remind the reader that by definition, a saddle connection is represented by a single geodesic segment joining a pair of conical points, and not by a broken line of geodesic segments.)

Choose, for example, a flat torus $\mathbb{R}^{2} /(\mathbb{Z} \oplus \mathbb{Z})$ as a surface $S$ and mark a generic pair of points $P_{0}, P_{1}$ on this torus. Let $\vec{v}_{0}:=\overrightarrow{P_{0} P_{1}}$. The set $V_{s c}(S) \subset \mathbb{R}^{2}$ is the square lattice of the form $\left\{\vec{v}_{0}+\vec{u}\right\}_{\vec{u} \in \mathbb{Z} \oplus \mathbb{Z} \text {. }}$

Similarly to the torus case, the set $V_{s c}(S)$ is a discrete subset of $\mathbb{R}^{2}$. However, when $S$ is different from the torus the map from the set of saddle connections on $S$ to $V_{s c}(S)$ is not injective: different saddle connections may have the same holonomy. We define the multiplicity of an element $\vec{v} \in V_{s c}(S)$ to be the number $p$ of distinct saddle connections $\gamma_{1}, \ldots, \gamma_{p}$ such that $\operatorname{hol}\left(\gamma_{i}\right)=\vec{v}$. For example, for the surface $S$ presented on the left of Figure 2 the vector $(0,1) \in V_{s c}(S)$ has multiplicity four: the saddle connections $\beta, \gamma, \gamma_{1}, \eta$ chosen with appropriate orientation have holonomy $(0,1)$.

In this example the saddle connections represented by $\beta, \gamma, \gamma_{1}, \eta$ are not pairwise homologous. As indicated above, the fact that their holonomy coincides is not generic; deforming the surface slightly we see that the corresponding saddle connections have different holonomy; see the right-hand-side picture at Figure 2 The situation is different with the saddle connections $\gamma$ and $\gamma_{1}$, for they are homologous. The right-hand-side picture at Figure 2 confirms that even after any small deformation of the surface the corresponding saddle connections share the same holonomy vector $\operatorname{hol}(\gamma)=\operatorname{hol}\left(\gamma_{1}\right)$.

From the measure-theoretical point of view Proposition 3.1 allows us to assume from now on that if two saddle connections $\beta, \gamma$ have the same holonomy, then $\gamma$ and $\beta$ are homologous. In particular, if $\beta$ joins distinct saddles $P_{1}, P_{2}$, then $\gamma$ joins the same pair of saddles. Since the surfaces as in the left-hand-side picture at Figure 2 form a set of measure zero we will ignore them in our considerations.

Somewhat surprisingly, higher multiplicity is very common even for a generic surface. For example, we will show that if the genus is at least 3, then counting separately the vectors of multiplicity one in $V_{s c}(S)$ of length at most $L$ and the vectors of multiplicity two, we get quadratic growth in $L$ for the number of elements of both multiplicities.

Multiplicity is not the only property distinguishing elements in $V_{s c}(S)$ : some of them correspond to closed saddle connections, other elements correspond to saddle connections joining distinct zeroes. We may also consider only those $\vec{v} \in V_{s c}(S)$, which correspond to saddle connections joining some particular pair of saddles. We refine our consideration slightly more specifying the following data.

Suppose that we have precisely $p$ homologous saddle connections $\gamma_{1}, \ldots, \gamma_{p}$ joining a zero $z_{1}$ of order $m_{1}$ to a zero $z_{2}$ of order $m_{2}$, see Figure 5 for a topological picture. All the $\gamma_{i}, 1 \leq i \leq p$ have the same holonomy (where the orientation of each $\gamma_{i}$ is from $z_{1}$ to $z_{2}$ ). By convention the cyclic order of the $\gamma_{i}$ at $z_{1}$ is clockwise in the orientation defined by the flat structure. Let the angle between $\gamma_{i}$ and $\gamma_{i+1}$ 
at $z_{1}$ be $2 \pi\left(a_{i}^{\prime}+1\right)$; let the angle between $\gamma_{i}$ and $\gamma_{i+1}$ at $z_{2}$ be $2 \pi\left(a_{i}^{\prime \prime}+1\right)$. We call this data the configuration

$$
\mathcal{C}=\left(m_{1}, m_{2}, a_{i}^{\prime}, a_{i}^{\prime \prime}\right)
$$

of the $p$ homologous saddle connections. If there is just a single saddle connection $(p=1)$ then $\mathcal{C}=\left(m_{1}, m_{2}\right)$.

Convention 3. We reserve the notion "configuration" for geometric types of possible collections of saddle connections, and not for the collections themselves.

Now given a surface $S$ and a configuration $\mathcal{C}$, let $V_{\mathcal{C}}(S) \subset V_{s c}(S)$ denote the vectors $\vec{v}$ in $\mathbb{R}^{2}$ such that there are precisely $p$ saddle connections $\gamma_{1}, \ldots, \gamma_{p}$ forming the configuration of the type $\mathcal{C}$ and having holonomy $\operatorname{hol}\left(\gamma_{i}\right)=\vec{v}$. We want to compute the number of collections $\left\{\gamma_{1}, \ldots, \gamma_{p}\right\}$ of the type $\mathcal{C}$ having holonomy vector of length at most $L$. In other words, we want to compute the asymptotics as $L \rightarrow \infty$ of the cardinality

$$
\left|V_{\mathcal{C}}(S) \cap B(L)\right|
$$

of intersection of the discrete set $V_{\mathcal{C}}(S)$ with the disc $B(L) \subset \mathbb{R}^{2}$ of radius $L$ centered at the origin.

Now consider the second problem of this paper: counting closed saddle connections.

We have a surface $S$ and a saddle connection $\gamma_{1}$ joining a zero $z_{1}$ to itself. There may be other saddle connections $\gamma_{2}, \ldots, \gamma_{m}$ having the same holonomy vector as $\gamma_{1}$. By the same argument as above we shall always assume that all such $\gamma_{i}$ are homologous.

Some of the $\gamma_{i}$ may start and end at the same zero $z_{1}$, the others may start and end at the other zeroes. Each $\gamma_{i}$ returns to its zero at some angle $\theta_{i}$ which is an odd multiple of $\pi$. Some of the $\gamma_{i}$ may bound cylinders filled with regular closed geodesics. A configuration $\mathcal{C}$ describes all these geometric data; this notion will be formalized in Section 11

We may have numerous collections of precisely $p$ homologous closed saddle connections corresponding to a configuration $\mathcal{C}$, i.e. defining the prescribed number of cylinders, prescribed angles $\theta_{i}$, etc. As in the first problem for a surface $S$ and configuration $\mathcal{C}$ let $V_{\mathcal{C}}(S)$ denote the vectors $\vec{v}$ in $\mathbb{R}^{2}$ such that the saddle connections in $\mathcal{C}$ have holonomy $\operatorname{hol}\left(\gamma_{1}\right)=\cdots=\operatorname{hol}\left(\gamma_{p}\right)=\vec{v}$ equal to that vector. Again we shall compute the asymptotics as $L \rightarrow \infty$ of

$$
\left|V_{\mathcal{C}}(S) \cap B(L)\right|,
$$

where $B(L)$ is the disc of radius $L$.

Theorem (A. Eskin, H. Masur; EMa). For either of the problems, given a configuration $\mathcal{C}$, there is a constant $c=c(\alpha, \mathcal{C})$ such that for almost all $S$ in any connected component of any stratum $\mathcal{H}_{1}(\alpha)$ one has

$$
\lim _{L \rightarrow \infty} \frac{\left|V_{\mathcal{C}}(S) \cap B(L)\right|}{\pi L^{2}}=c(\alpha, \mathcal{C}) .
$$

The constant $c(\alpha, \mathcal{C})$ depends only on the connected component of the stratum and on the configuration $\mathcal{C}$.

Versions of the above theorems where convergence a.e. is replaced by convergence in $L^{1}$ are proved in $\mathrm{Ve} 4$. Thus the growth rate is quadratic for a generic surface. What is perhaps surprising is that this formula says that the growth rate 
is quadratic for a generic surface even for multiple homologous saddle connections. By contrast, a generic surface does not have any pairs of saddle connections that determine vectors in the same direction but with different lengths, see Proposition 7.4 at the end of Section 7

Remark 3.2. The issue of higher multiplicity and specifying the angles is not explicitly addressed in EMa. However, the set $V_{\mathcal{C}}(S)$ defined above, satisfies all of the axioms of that paper, so the asymptotic formula from [EMa] is applicable to the problems formulated above.

One of the main results of this paper is a description of all possible configurations $\mathcal{C}$ for any connected component of any stratum, and an evaluation of the corresponding constants $c(\alpha, \mathcal{C})$.

We note that the lists of configurations and the values of the constants have been verified numerically. Beside direct considerations of flat surfaces we used the following implicit experiments. A formula based on the formula of Kontsevich (see $[\mathrm{Ko}$ ) expresses the sum of the Lyapunov exponents of the Teichmüller geodesic flow on each connected component of every stratum $\mathcal{H}(\alpha)$ in terms of some rational function of $\alpha$ and of some specific linear combination of the constants $c(\alpha, \mathcal{C})$ for the corresponding component of $\mathcal{H}(\alpha)$. These Lyapunov exponents have been computed numerically and the constants achieved in this paper are consistent with these exponents and with Kontsevich's formula.

3.3. Siegel-Veech Formula. Fix a topological type of a configuration $\mathcal{C}$. To every flat surface $(S, \omega)$ in $\mathcal{H}_{1}(\alpha)$ we can associate a discrete set $V_{\mathcal{C}}(S) \subset \mathbb{R}^{2}$. This enables us to construct an operator $f \mapsto \hat{f}$ from the space of integrable functions with compact support in $\mathbb{R}^{2}$ to functions on $\mathcal{H}_{1}(\alpha)$. We define $\hat{f}$ as

$$
\hat{f}(S):=\sum_{\vec{v} \in V_{\mathcal{C}}(S)} f(\vec{v}) .
$$

Averaging $\hat{f}$ over the entire moduli space we get a functional on the space of functions with compact support in $\mathbb{R}^{2}$. In $\mathrm{Ve} 4$ it was proved that this functional is $S L(2, \mathbb{R})$-invariant which implies the following Siegel-type formula. For each configuration $\mathcal{C}$ and for each connected component $\mathcal{H}_{1}(\alpha)$ of each stratum the average of $\hat{f}$ over $\mathcal{H}_{1}(\alpha)$ and the average of $f$ over $\mathbb{R}^{2}$ coincide up to a multiplicative factor const $(\mathcal{C})$ which depends only on the configuration type and on the connected component of the stratum. The formula

$$
\frac{1}{\operatorname{Vol}\left(\mathcal{H}_{1}(\alpha)\right)} \int_{\mathcal{H}_{1}(\alpha)} \hat{f}(S) d \operatorname{vol}(S)=\operatorname{const}(\mathcal{C}) \int_{\mathbb{R}^{2}} f
$$

was called in EMa the Siegel-Veech formula. It was shown in EMa that the Siegel-Veech constant const $(\mathcal{C})$ in the formula above and the $c(\mathcal{C})$ in equation (4) coincide. Thus, one of the major goals of this paper can be thought of as finding the constant in the Siegel-Veech formula (5).

The strategy for evaluating $c(\mathcal{C})=\operatorname{const}(\mathcal{C})$ consists of choosing a convenient function $f$ for which the complicated integral on the left in (5) can be computed as explicitly as possible. As such a "convenient function" we take the characteristic function $f_{\varepsilon}$ of the disc $B(\varepsilon)$ centered at the origin of $\mathbb{R}^{2}$, where $\varepsilon$ is the radius of the disc. The integral on the right in equation (5) is equal to $\pi \varepsilon^{2}$. The integral on the left gives the average number of configurations of the type $\mathcal{C}$ having holonomy 
vector shorter than $\varepsilon$. We cannot compute this average explicitly for any given $\varepsilon$. However, we can compute the leading term of order $\varepsilon^{2}$ in the asymptotics of the left-hand-side of (5) as $\varepsilon \rightarrow 0$. This will be sufficient for our purposes. To compute this leading term we use the idea which we illustrate in the simplest case, when the configuration $\mathcal{C}$ consists of a single saddle connection joining two distinct saddles $z_{1}, z_{2}$. The general case, as well as a rigorous proof of equation (6) below is treated in Section 7

Denote the support of $\hat{f}_{\varepsilon}$ by $\mathcal{H}_{1}^{\varepsilon}(\alpha, \mathcal{C})$. This is the subset of flat surfaces $S$, which possess at least one short saddle connection $\gamma$ of multiplicity one joining the chosen zeroes $z_{1}, z_{2}$. Saying "short" we mean that $|\vec{v}| \leq \varepsilon$, where $\vec{v}=h o l(\gamma)$. This space is not compact: for any compact subset $K$ of $\mathcal{H}_{1}(\alpha)$ the length of the shortest saddle connection on any flat surface $S \in K$ is bounded from below. The space $\mathcal{H}_{1}^{\varepsilon}(\alpha, \mathcal{C})$ is difficult to analyze since a surface $S \in \mathcal{H}_{1}^{\varepsilon}(\alpha, \mathcal{C})$ may have short saddle connections different from $\gamma$, possibly even many saddle connections that intersect.

To simplify our task we decompose $\mathcal{H}_{1}^{\varepsilon}(\alpha, \mathcal{C})$ into two complementary subsets: a "thick" and a "thin" part: $\mathcal{H}_{1}^{\varepsilon}(\alpha, \mathcal{C})=\mathcal{H}_{1}^{\varepsilon, \text { thick }}(\alpha, \mathcal{C}) \sqcup \mathcal{H}_{1}^{\varepsilon, \text { thin }}(\alpha, \mathcal{C})$. The thick part $\mathcal{H}_{1}^{\varepsilon, \text { thick }}(\alpha, \mathcal{C})$ consists of surfaces $S$ having exactly one saddle connection $\gamma$ shorter than $\varepsilon$; moreover, we require that this $\gamma$ joins the two chosen zeroes $z_{1}$ and $z_{2}$. The thin part $\mathcal{H}_{1}^{\varepsilon, \text { thin }}(\alpha, \mathcal{C})$ consists of surfaces $S$ having at least one short saddle connection $\gamma$ as above and at least one other short saddle connection $\beta$ nonhomologous to $\gamma$. Here $\beta$ is an arbitrary short saddle connection, not necessarily joining $z_{1}$ to $z_{2}$, possibly closed.

The function $\hat{f}_{\varepsilon}$ is equal to zero outside of $\mathcal{H}_{1}^{\varepsilon}(\alpha, \mathcal{C})$. By definition of the thick part, the value of $\hat{f}_{\varepsilon}$ on any $S \in \mathcal{H}_{1}^{\varepsilon, t h i c k}(\alpha, \mathcal{C})$ is identically 1 . Finally, for $S \in$ $\mathcal{H}_{1}^{\varepsilon, t h i n}(\alpha, \mathcal{C})$ we have $\hat{f}_{\varepsilon}(S) \geq 1$. Thus, we get the following representation of the integral above:

$$
\int_{\mathcal{H}_{1}(\alpha)} \hat{f}_{\varepsilon}(S) d \operatorname{vol}(S)=\int_{\mathcal{H}_{1}^{\varepsilon, t h i c k}(\alpha, \mathcal{C})} d \operatorname{vol}(S)+\int_{\mathcal{H}_{1}^{\varepsilon, t h i n}(\alpha, \mathcal{C})} \hat{f}_{\varepsilon}(S) d \operatorname{vol}(S)
$$

The first term in this sum is just the volume of the thick part. Even though $\hat{f}_{\varepsilon}$ is unbounded on the thin part, its measure is so small that the integral of $\hat{f}$ over the thin part is negligible: it is of the order $o\left(\varepsilon^{2}\right)$. We prove this statement in Section 7 using a nontrivial upper bound for $\hat{f}_{\varepsilon}$ found in EMa. The fact that the thin part is small implies also that $\operatorname{Vol}\left(\mathcal{H}_{1}^{\varepsilon}(\alpha, \mathcal{C})\right)=\operatorname{Vol}\left(\mathcal{H}_{1}^{\varepsilon, \text { thick }}(\alpha, \mathcal{C})\right)+o\left(\varepsilon^{2}\right)$. Hence, we get the following key statement:

Proposition 3.3. For any connected component of any stratum $\mathcal{H}(\alpha)$ and for any configuration $\mathcal{C}$ the following limit exists and is equals to the corresponding SiegelVeech constant:

$$
c(\mathcal{C})=\lim _{\varepsilon \rightarrow 0} \frac{1}{\pi \varepsilon^{2}} \frac{\operatorname{Vol}\left(\mathcal{H}_{1}^{\varepsilon}(\alpha, \mathcal{C})\right)}{\operatorname{Vol}\left(\mathcal{H}_{1}(\alpha)\right)}
$$

We prove this Proposition in Section 7 Formula (6) explains why one of the principal goals of this paper is the calculation of the asymptotics of the volume $\operatorname{Vol}\left(\mathcal{H}_{1}^{\varepsilon}(\alpha, \mathcal{C})\right)$ for any connected component of any stratum and for any configuration $\mathcal{C}$ of homologous saddle connections admissible for this component.

3.4. Principal Boundary of the Moduli Spaces. Now as one lets $\varepsilon \rightarrow 0$, the flat surfaces in the thick part degenerate to simpler surfaces. In the case of a 
single saddle connection joining a pair of distinct zeroes the zeroes coalesce to a higher order zero giving a surface in a stratum in the same genus; the complex dimension of the resulting stratum decreases by one with respect to the initial one. In general, collapsing all saddle connections from a configuration $\mathcal{C}$ to a single point we obtain a surface from more degenerate stratum; the surface is disconnected if the configuration has multiplicity two or more. We shall say that the resulting surface (connected or not) belongs to the principal boundary of the original stratum. Each configuration $\mathcal{C}$ will determine a "face" of this principal boundary. A major part of this paper then will be to describe this principal boundary, an object of independent interest.

Conversely, given a flat surface $S^{\prime}$ in the principal boundary, we will describe a set of surgeries on $S^{\prime}$ that allow us to recover surfaces $S$ in the thick part. The first surgery is a splitting apart of a higher order zero into simpler zeroes that is the opposite of coalescing. The second one is a slit and gluing construction which is the opposite of the degeneration of multiple saddle connections. To construct surfaces with homologous closed geodesics, we shall also need a pair of constructions, called the figure eight and creating a pair of holes construction.

The above constructions lead to the following picture. Let $\mathcal{C}$ be a configuration of homologous saddle connections joining two distinct points. We shall prove that the thick part $\mathcal{H}_{1}^{\varepsilon, t h i c k}(\alpha, \mathcal{C})$ has the structure of a (ramified) covering over a direct product $\mathcal{H}_{1}\left(\alpha^{\prime}\right) \times B(\varepsilon)$ of the corresponding stratum of surfaces obtained after degeneration of $\mathcal{C}$ and a two-dimensional disc $B(\varepsilon)$ of radius $\varepsilon$. Moreover, the measure on the thick part $\mathcal{H}_{1}^{\varepsilon, t h i c k}(\alpha, \mathcal{C})$ is just the product of corresponding measures on the two factors $\mathcal{H}_{1}\left(\alpha^{\prime}\right)$ and $B(\varepsilon)$. The degree of the covering (the combinatorial constant) $M$ corresponds to the number of ways to perform the surgeries on $S^{\prime}$ to obtain $S$. Hence, we shall obtain the following answer in all cases

$$
\operatorname{Vol}\left(\mathcal{H}_{1}^{\varepsilon}(\alpha, \mathcal{C})\right)=M \cdot \pi \varepsilon^{2} \cdot \operatorname{Vol}\left(\mathcal{H}_{1}\left(\alpha^{\prime}\right)\right)+o\left(\varepsilon^{2}\right) .
$$

Applying (6) we then are able to prove the following result

$$
c(\mathcal{C})=M \cdot \frac{\operatorname{Vol}\left(\mathcal{H}_{1}\left(\alpha^{\prime}\right)\right)}{\operatorname{Vol}\left(\mathcal{H}_{1}(\alpha)\right)}
$$

Analogous considerations can be applied to a configuration $\mathcal{C}$ of closed saddle connections. Since we are interested in the answers for all connected components of the strata we shall need in certain situations to pay attention to parity of spin structures and hyperellipticity.

One can think of every stratum as an analogue of a complex polyhedron; the boundary of the polyhedron has "faces" of complex codimension 1, "edges" of complex codimension 2, etc. A collection of all admissible types $\mathcal{C}$ of configurations of homologous saddle connections describes all generic degenerations of our surfaces, that is all possible "faces" of the boundary. Thus, though we do not construct a compactification of the strata $\mathcal{H}_{1}(\alpha)$ in full generality we describe the principal boundary of the strata $\mathcal{H}_{1}(\alpha)$

Remark 3.4. One can consider these same problems in the context of quadratic differentials. To avoid overloading the paper we prefer to treat them in a subsequent one. 
Remark 3.5. A list of the geometric types of admissible configurations $\mathcal{C}$ is shared by almost all surfaces in any connected component of any stratum. However, this list varies when we change the stratum, or a connected component of the stratum. Thus, such lists can be used as an invariant of a connected component. There are other orbifolds in the moduli space of Abelian differentials invariant under the $S L(2, \mathbb{R})$ action; they have their own lists of geometric types of admissible configurations (understood more generally). These lists have proved to be important invariants of such orbifolds: in $\mathrm{HuS}$ they are used to give an example of different Veech surfaces (with different Teichmüller discs) sharing isomorphic Veech groups.

3.5. Arithmetic of Siegel-Veech Constants. As remarked earlier the number of (homotopy classes) of primitive closed geodesics of length at most $L$ on a flat torus of area one (considered up to a symmetry of the torus) is approximately

$\frac{3}{\pi^{2}} \cdot \pi L^{2}$. The number of geodesic segments of length at most $L$ joining a generic pair of distinct points $P_{1}, P_{2}$ on the same torus is approximately $1 \cdot \pi L^{2}$.

The general Siegel — Veech constants $c(\mathcal{C})$ share the same arithmetic property! Those Siegel - Veech constants, which correspond to configurations of closed saddle connections always have the form of a rational divided by $\pi^{2}$; those Siegel - Veech constants, which correspond to configurations of saddle connections joining distinct points are always rational.

We have no simple geometric explanation of this phenomenon. It rather follows directly from (7), the fact that the combinatorial factor $M$ is by its meaning always rational (mostly integer) and the fact that the volume of any connected component of any stratum $\mathcal{H}_{1}(\alpha)$ of Abelian differentials is a rational multiple of $\pi^{2 g}$, where $g$ is the genus of the surface. This latter fact was conjectured by M. Kontsevich and proved by A. Eskin and A. Okounkov, who, actually, calculated these volumes (see EOk for connected strata). This property of the volume is true not only for primitive strata, but for strata of surfaces with marked points and even for strata of disconnected surfaces.

\section{REAders Guide}

In the first half of the paper we solve these problems for saddle connections joining distinct zeroes. We describe the multiplicity one case first, because it is the easiest one and because it illustrates the computations. This is done in Sections 8.28 .3 We then describe the principal boundary in the higher multiplicity case, describing the slit and gluing constructions and again give the constants. This is done in Sections 9.1 9.2 and 9.5 Sections 10.110.4 as well as the corresponding preliminaries from Section 5.1 are devoted to describing the constants in the cases of strata with several components and could be skipped by the reader interested only in the general thrust of the paper.

In the second half of the paper we consider the case of closed saddle connections. We describe the principal boundary using the creating a pair of holes and figure eight constructions. The reader interested only in the structure of the principal boundary may consult Sections 11, 12 The computation of the combinatorial factor is performed in Section 13.3 and the computation of the constant $c$ is given in Section 13.1 The rest of Section 13 is devoted to numerous examples and Section 14 is devoted to the special case of strata with several connected components. 
We are illustrating all constructions by numerous examples. All possible strata and all possible configurations in genera $g=2$ and $g=3$ and all related computations are presented as the examples in the main body of the text. The complete list of admissible configurations in genus $g=4$, and the values of the corresponding constants are presented in the Appendix. We also present in the Appendix the values of the constants for the two distinguished strata $\mathcal{H}(1, \ldots, 1)$ and $\mathcal{H}^{\text {hyp }}(g-1, g-1)$ up to genus $g=30$.

\section{Part 0. Structure of the Strata}

\section{Connected Components of the Strata}

5.1. Parity of a Spin Structure. As noted earlier, the strata $\mathcal{H}_{1}(\alpha)$ are not necessarily connected. To classify the components one needs the notion of parity of spin structure. Consider a smooth simple curve $\beta$ on the flat surface $S$ which does not contain any zeroes. We define the index $\operatorname{ind}(\beta) \in \mathbb{N}$ of the vector field tangent to $\beta$ to be the degree of the corresponding Gauss map: the total change of the angle between the vector tangent to the curve and the vector tangent to the horizontal foliation is $2 \pi \cdot \operatorname{ind}(\beta)$.

Now take a symplectic homology basis $\left\{a_{i}, b_{i}\right\}, i=1, \ldots, g$, such that the intersection matrix has the canonical form: $a_{i} \circ a_{j}=b_{i} \circ b_{j}=0, a_{i} \circ b_{j}=\delta_{i j}, 1 \leq i, j \leq g$. Though such basis is not unique, traditionally it is called a canonical basis. Consider a collection of smooth closed curves representing the chosen basis. Denote them by the same symbols $a_{i}, b_{i}$.

When all zeroes of the Abelian differential $\omega$ corresponding to the flat structure have only even degrees we can define the parity of the spin structure $\phi(S)$ as

$$
\phi(S):=\sum_{i=1}^{g}\left(\operatorname{ind}\left(a_{i}\right)+1\right)\left(\operatorname{ind}\left(b_{i}\right)+1\right) \quad(\bmod 2)
$$

It follows from the results of D.Johnson [J] that the parity of the spin structure $\phi(S)$ does not depend on the choice of representatives, nor on the choice of the canonical homology bases.

Moreover, it follows from the results of M.Atiyah [At that $\phi(S)$ is invariant under continuous deformations of the flat structure of the translation surface, (see KonZo for details).

Since we do not need the notion of a spin structure itself, we do not discuss it in this paper; the reader may consider the notion "parity of the spin structure" as a single term. Note that it is applicable only to those flat structures $\omega$ which have a set of zeros $\alpha$ containing even numbers only.

5.2. Classification of Connected Components of the Strata. The components are classified as follows (see $\mathrm{KonZo}$ ).

Consider the general case, when $g>3$, and $\alpha$ is different from two exceptional cases: $\alpha \neq(2 g-2), \alpha \neq(g-1, g-1)$. Under these assumptions the stratum $\mathcal{H}_{1}(\alpha)$ is connected whenever collection $\alpha$ contains an odd number. When $g>3$, $\alpha \neq(2 g-2), \alpha \neq(g-1, g-1)$, and $\alpha$ has only even elements, there are exactly two 
connected components corresponding to even and odd spin structures. We shall denote them by $\mathcal{H}_{1}^{\text {even }}(\alpha)$ and $\mathcal{H}_{1}^{\text {odd }}(\alpha)$ correspondingly.

The strata $\mathcal{H}_{1}(2 g-2)$ and $\mathcal{H}_{1}(g-1, g-1)$ have in addition a special component - the hyperelliptic one. It consists of flat structures on hyperelliptic surfaces. In both cases the hyperelliptic involution $\tau$ sends the flat structure $\omega$ to $-\omega$; for $\mathcal{H}_{1}(g-1, g-1)$ we also require that $\tau$ interchanges two zeroes of $\omega$.

For $g>3$ the stratum $\mathcal{H}_{1}(2 g-2)$ has three connected components: the hyperelliptic one $-\mathcal{H}_{1}^{\text {hyp }}(2 g-2)$, and two nonhyperelliptic components $-\mathcal{H}_{1}^{\text {even }}(2 g-2)$ and $\mathcal{H}_{1}^{\text {odd }}(2 g-2)$ corresponding to even and odd spin structures. For even $g>3$ the stratum $\mathcal{H}_{1}(g-1, g-1)$ has two connected components: the hyperelliptic one, and the nonhyperelliptic one $\mathcal{H}_{1}^{\text {nonhyp }}(g-1, g-1)$. For odd $g>3$ the stratum $\mathcal{H}_{1}(g-1, g-1)$ has three connected components: the hyperelliptic one, denoted by $\mathcal{H}_{1}^{\text {hyp }}(g-1, g-1)$, and two nonhyperelliptic ones $-\mathcal{H}_{1}^{\text {even }}(g-1, g-1)$ and $\mathcal{H}_{1}^{\text {odd }}(g-1, g-1)$ corresponding to even and odd spin structures.

Genera 2 and 3 are special. In genus 2, all flat structures are hyperelliptic, so each of the $\mathcal{H}_{1}(2)$ and $\mathcal{H}_{1}(1,1)$ have one component which is hyperelliptic. In genus 3 , only hyperelliptic flat structures may have even parity of the spin structure. Thus $\mathcal{H}_{1}(4)$ and $\mathcal{H}_{1}(2,2)$ each have two connected components, the hyperelliptic one and the one with odd spin structure. The other strata in genus 3 are connected.

The flat surfaces from hyperelliptic strata have the following parity of the spinstructure (see KonZo]). The parity of the spin structure determined by a flat structure from the hyperelliptic component $S \in \mathcal{H}_{1}^{\text {hyp }}(2 g-2)$ equals

$$
\phi(S)=\left[\frac{g+1}{2}\right](\bmod 2)
$$

where the square brackets denote the integer part of a number. The parity of the spin structure determined by a flat structure $\omega$ from the hyperelliptic component $\omega \in \mathcal{H}_{1}^{\text {hyp }}(g-1, g-1)$, for odd genera $g$ equals

$$
\phi(S)=\left(\frac{g+1}{2}\right)(\bmod 2) \quad \text { for odd } g
$$

\section{Nonprimitive Strata}

6.1. Strata of Surfaces with Marked Points. In this paper we shall often consider the strata $\mathcal{H}_{1}(\alpha)$ of surfaces $S=(M, \omega)$ where we not only fix the zeroes of the Abelian differential $\omega$, but we also mark one or more regular points on the surface. Say, $\mathcal{H}_{1}(3,1,0)$ will denote the surfaces of genus $g=3$ endowed with an Abelian differential with zeroes of orders 3 and 1 having one additional marked point ("zero of order 0 of the Abelian differential").

Throughout this paper we assume that all zeroes and all marked points are always numbered. Thus a stratum with one or more marked points has the natural structure of a fiber bundle over the corresponding stratum without marked points with a direct product (minus diagonals) of several copies of the translation surface as a fiber, where the number of copies equals the number of marked points. For example, the universal curve $\mathcal{H}_{1}(3,1,0)$ fibers over $\mathcal{H}_{1}(3,1)$ with a fiber $S$.

In particular, there is the following formula for the dimension of the strata with marked points. Let $g$ be the genus and let $\operatorname{card}(\alpha)$ denote the number of entries in 
$\alpha$. Suppose that $0 \notin \alpha$. Then

$$
\operatorname{dim}_{\mathbb{C}} \mathcal{H}(\alpha, \underbrace{0, \ldots, 0}_{n})=\operatorname{dim}_{\mathbb{C}} \mathcal{H}(\alpha)+n=2 g-1+\operatorname{card}(\alpha)+n
$$

Convention 4. By convention we always mark a point on a flat torus. We denote the corresponding stratum $\mathcal{H}(0)$.

Note that $\operatorname{dim}_{\mathbb{C}} \mathcal{H}(0)=2$ which matches formula (10).

The natural measure on the stratum with marked points disintegrates into a product measure, where the measure along the fiber is just the Lebesgue measure on $S$ (correspondingly product of several copies of $S$ ) induced by the flat metric on $S$, and the measure on the base is the natural measure on the corresponding stratum taken without marked points.

Recall that from the point of view of volumes we have confined ourselves to the subspaces $\mathcal{H}_{1}(\alpha)$ of the strata for which the area of every surface $S$ (measured in the flat metric) is equal to one. The observation above implies that volumes of the strata with marked points coincide with the volumes of the corresponding strata without marked points:

$$
\operatorname{Vol}\left(\mathcal{H}_{1}(\alpha)\right)=\operatorname{Vol}\left(\mathcal{H}_{1}(\alpha, 0)\right)=\operatorname{Vol}\left(\mathcal{H}_{1}(\alpha, 0,0)\right)=\ldots
$$

6.2. Strata of Disconnected Surfaces. It will be convenient to consider sometimes the strata $\mathcal{H}\left(\alpha^{\prime}\right)=\prod_{i=1}^{p} \mathcal{H}\left(\alpha_{i}^{\prime}\right)$, of closed flat surfaces $S$ having $p$ connected components $S_{1} \sqcup \cdots \sqcup S_{p}$ of prescribed types.

Convention 5. Using notation $\alpha^{\prime}=\sqcup_{i=1}^{p} \alpha_{i}^{\prime}$ for the strata $\mathcal{H}\left(\alpha^{\prime}\right)$ of disconnected surfaces we assume that we keep track of how $\alpha^{\prime}$ is partitioned into collections $\alpha_{i}^{\prime}$.

We shall need the expressions for the volume element and for the total volume of such strata.

We write $S_{i}=r_{i} S_{i}^{\prime}$, where area $\left(S_{i}^{\prime}\right)=1$. Then area $\left(S_{i}\right)=r_{i}^{2}$. Let $d_{i}:=$ $\operatorname{dim}_{\mathbb{R}} \mathcal{H}\left(\alpha_{i}^{\prime}\right)$; let $d:=\operatorname{dim}_{\mathbb{R}} \mathcal{H}\left(\alpha^{\prime}\right)=\sum_{i=1}^{p} d_{i}$; let $d \nu_{i}^{\prime}$ be the volume element on the stratum $\mathcal{H}\left(\alpha_{i}^{\prime}\right)$ (which should not be confused with the volume element on a "unit hyperboloid" $\mathcal{H}_{1}\left(\alpha_{i}^{\prime}\right)$ in the same stratum, see Convention 21). We have

$$
d \nu(S)=\prod_{i=1}^{p} d \nu_{i}^{\prime}\left(S_{i}\right)=\prod_{i=1}^{p}\left(r_{i}^{d_{i}-1} d r_{i}\right) \prod_{i=1}^{p} d \operatorname{vol}^{\prime}\left(S_{i}^{\prime}\right)
$$

Let $D(1)$ be the unit ball $r_{1}^{2}+\cdots+r_{p}^{2} \leq 1$; set

$$
W=\prod_{i=1}^{p} \operatorname{Vol}\left(\mathcal{H}_{1}\left(\alpha_{i}^{\prime}\right)\right)
$$

Then,

$$
\nu\left(C\left(\mathcal{H}_{1}\left(\alpha^{\prime}\right)\right)=W \cdot \int_{D(1)} \prod_{j=1}^{p} r_{j}^{d_{j}-1} d r_{j}\right.
$$

We now make the change of variable $x_{i}=r_{i}^{2}$ to evaluate the integral. For each $i$, let $b_{i}=d_{i} / 2-1$, so that $r_{i}^{d_{i}-1} d r_{i}=(1 / 2) x_{i}^{b_{i}} d x_{i}$. Then the above integral becomes

$$
\nu\left(C\left(\mathcal{H}_{1}\left(\alpha^{\prime}\right)\right)=W \cdot \frac{1}{2^{p}} \int_{\sum_{i} x_{i} \leq 1} x_{1}^{b_{1}} \ldots x_{p}^{b_{p}} d x_{1} \ldots d x_{p}\right.
$$


where now we integrate over the standard simplex. Repeated application of the identity

yields

$$
\int_{0}^{u} x^{a}(u-x)^{b} d x=\frac{a ! b !}{(a+b+1) !} u^{a+b+1}
$$

$$
\nu\left(C\left(\mathcal{H}_{1}\left(\alpha^{\prime}\right)\right)=W \cdot \frac{1}{2^{p}} \frac{b_{1} ! \ldots b_{p} !}{\left(b_{1}+\cdots+b_{p}+p\right) !}\right.
$$

Since $b_{1}+\cdots+b_{p}+p=\sum\left(d_{i} / 2\right)=d / 2$ we obtain

$$
\nu\left(C\left(\mathcal{H}_{1}\left(\alpha^{\prime}\right)\right)=\frac{W}{2^{p}} \cdot \frac{\left(\frac{d_{1}}{2}-1\right) ! \ldots\left(\frac{d_{p}}{2}-1\right) !}{\left(\frac{d}{2}\right) !}\right.
$$

Hence,

$$
\begin{array}{r}
\operatorname{Vol}\left(\mathcal{H}_{1}\left(\alpha^{\prime}\right)\right)=d \cdot \nu\left(C\left(\mathcal{H}_{1}\left(\alpha^{\prime}\right)\right)=\frac{2 \cdot \frac{d}{2} \cdot W}{2^{p}} \cdot \frac{\left(\frac{d_{1}}{2}-1\right) ! \ldots\left(\frac{d_{p}}{2}-1\right) !}{\left(\frac{d}{2}\right) !}=\right. \\
=\frac{1}{2^{p-1}} \cdot \frac{\left(\frac{d_{1}}{2}-1\right) ! \ldots\left(\frac{d_{p}}{2}-1\right) !}{\left(\frac{d}{2}-1\right) !} \cdot \prod_{i=1}^{p} \operatorname{Vol}\left(\mathcal{H}\left(\alpha_{i}^{\prime}\right)\right)
\end{array}
$$

Repeating literarily the same arguments we obtain the corresponding formula for the volume elements:

$$
d \mathrm{vol}=\frac{1}{2^{p-1}} \cdot \frac{\left(\frac{d_{1}}{2}-1\right) ! \ldots\left(\frac{d_{p}}{2}-1\right) !}{\left(\frac{d}{2}-1\right) !} \cdot d \operatorname{vol}_{1}^{\prime} \cdots d \operatorname{vol}_{p}^{\prime}
$$

\section{Thick-Thin Decomposition, Volume Estimates, and Computation of the Siegel-Veech Constants}

In this section we prove Proposition 3.3 and justify the key Formula( 60) for the Siegel-Veech constant. We also describe more precisely the structure of the thick - thin decomposition of neighborhoods of the cusps $\mathcal{H}_{1}^{\varepsilon}(\alpha, \mathcal{C})$. Our estimates of the volumes of the thick and the thin part are based on the following result:

Lemma 7.1 (H. Masur, J. Smillie). There is a constant $M$ such that for all $\varepsilon, \kappa>0$ the subset of $\mathcal{H}_{1}(\alpha)$ consisting of those flat surfaces, which have a saddle connection of length at most $\varepsilon$, has volume at most $M \varepsilon^{2}$. The volume of the set of flat surfaces with a saddle connection of length at most $\varepsilon$ and a nonhomologous saddle connection with length at most $\kappa$ is at most $M \varepsilon^{2} \kappa^{2}$.

Proof. The proof is contained in the proof of Theorem 10.3 in $[\mathrm{MS}$.

As we have seen in Section 3.3 it is convenient to decompose the set $\mathcal{H}_{1}^{\varepsilon}(\alpha, \mathcal{C})$, which plays the role of a neighborhood of the "cusp" corresponding to the configuration $\mathcal{C}$, into two disjoint subsets: the "thick" and the "thin" part, $\mathcal{H}_{1}^{\varepsilon}(\alpha, \mathcal{C})=$ $\mathcal{H}_{1}^{\varepsilon, \text { thick }}(\alpha, \mathcal{C}) \sqcup \mathcal{H}_{1}^{\varepsilon, \text { thin }}(\alpha, \mathcal{C})$. Sometimes it will be convenient to vary slightly this partition making the thin part a bit larger or a bit smaller depending on the consideration. This does not affect the sense of the thick - thin decomposition, but simplifies the proofs. This variations can be described as follows. We use the parameter $\varepsilon, 0<\varepsilon<1$ to bound the length of the shortest saddle connection. We introduce the bound $\kappa:=\lambda \cdot \varepsilon^{r}$ for the length of the shortest saddle connection nonhomologous to the first one. Here the parameters $\lambda$ and $r$ satisfy the following conditions: $\lambda \geq 1,0<r \leq 1$, which guaranties $\kappa(\lambda, r) \geq \varepsilon$ for all $\lambda$ and $r$. 
The subset $\mathcal{H}_{1}^{\varepsilon}(\alpha, \mathcal{C}) \subset \mathcal{H}_{1}(\alpha)$ is comprised of those surfaces which have at least one collection of short (shorter than $\varepsilon$ ) homologous saddle connections of the type $\mathcal{C}$. The thin part $\mathcal{H}_{1}^{\varepsilon, \text { thin }}(\alpha, \mathcal{C})$ of this subset consists of surfaces $S$ having at least one additional saddle connection of any type shorter than $\kappa$.

The complement to the thin part in $\mathcal{H}_{1}^{\varepsilon}(\alpha, \mathcal{C})$, the thick part $\mathcal{H}_{1}^{\varepsilon, \text { thick }}(\alpha, \mathcal{C})$, consists of surfaces $S$ having exactly one collection of short homologous saddle connections. This collection is necessarily of the type $\mathcal{C}$; the saddle connections from this collection (which are all of the same length) are shorter then $\varepsilon$; any other saddle connection on $S$ is longer then $\kappa$.

Lemma 7.1 implies the following immediate corollary.

Corollary 7.2. For any connected component of any stratum $\mathcal{H}(\alpha)$, any configuration $\mathcal{C}$ and any choice of parameters $\lambda, r$ defining the thick - thin decomposition we have

$$
\operatorname{Vol}\left(\mathcal{H}_{1}^{\varepsilon}(\alpha, \mathcal{C})\right)=\operatorname{Vol}\left(\mathcal{H}_{1}^{\varepsilon, t h i c k}(\alpha, \mathcal{C})\right)+o\left(\varepsilon^{2}\right) .
$$

To prove Formula( [6) it remains to prove the following Lemma.

Lemma 7.3. Let $f$ be the characteristic function of a disc of radius $\varepsilon$ centered at the origin of $\mathbb{R}^{2}$. For any connected component of any stratum $\mathcal{H}(\alpha)$, any configuration $\mathcal{C}$ and for an appropriate choice of parameters $\lambda, r$ defining the thick-thin decomposition the integral of the function $\hat{f}_{\mathcal{C}}$ over the thin part is negligible:

$$
\int_{\mathcal{H}_{1}^{\varepsilon, \text { thin }}(\alpha, \mathcal{C})} \hat{f_{\mathcal{C}}}(S) d \operatorname{vol}(S)=o\left(\varepsilon^{2}\right)
$$

Proof. Recall that the nonnegative function $\hat{f}_{\mathcal{C}}(S)$ counts only those saddle collections which are arranged in the configuration $\mathcal{C}$; it can be defined as the cardinality of the intersection of the discrete set $V_{\mathcal{C}}(S)$ with the disc $B(\varepsilon)$.

$$
\hat{f}_{\mathcal{C}}(S):=\left|V_{\mathcal{C}}(S) \cap B(\varepsilon)\right|
$$

Consider the analogous function

$$
\hat{f}(S):=\left|V_{s c}(S) \cap B(\varepsilon)\right|
$$

which counts all short saddle connections (without multiplicity) regardless of which configuration they correspond to. Clearly $\hat{f} \geq \hat{f}_{\mathcal{C}}$ since $V_{\mathcal{C}}(S) \subset V_{s c}$. Thus, it is sufficient to prove the above Lemma for the function $\hat{f}$. We use the following estimate proved in EMa.

Theorem (A. Eskin, H. Masur). Let $l(S)$ denote the length of the shortest saddle connection on $S \in \mathcal{H}_{1}(\alpha)$. For any connected component of any stratum $\mathcal{H}_{1}(\alpha)$ there exist constants $c^{\prime}$ and $0<\delta<1$, depending only on the stratum, so that for any $S \in \mathcal{H}_{1}(\alpha)$ for which $l(S)$ is sufficiently small, the following bound is valid:

$$
\hat{f}(S) \leq \frac{c^{\prime}}{(l(S))^{1+\delta}}
$$

We now can complete the proof of Lemma 7.3 Choose any $0<r \leq 1$ so that

$$
2 r>1+\delta ;
$$


and let $\lambda=1$. This choice of $\lambda, r$ gives $\kappa=1 \cdot \varepsilon^{r}$; consider the thick-thin decomposition corresponding to this choice of parameters. To prove the estimate

$$
\int_{\mathcal{H}_{1}^{\varepsilon, t h i n}(\alpha, \mathcal{C})} \hat{f}(S) d \operatorname{vol}(S)=o\left(\varepsilon^{2}\right)
$$

we decompose the set $\mathcal{H}_{1}^{\varepsilon, t h i n}(\alpha, \mathcal{C})$ into a disjoint union of subsets $U_{n}$, such that the shortest saddle connection for $S$ in $U_{n}$ has length $l(S)$ satisfying $\varepsilon / 2^{n+1}<$ $l(S) \leq \varepsilon / 2^{n}$, where $n$ is a non-negative integer. Since by definition, on each surface in $U_{n}$ there is a saddle connection with length between $\varepsilon / 2^{n+1}$ and $\varepsilon / 2^{n}$ and a nonhomologous saddle connection with length at most $\kappa=\varepsilon^{r}$, by Lemma 7.1 there is a constant $M$ so that the measure of $U_{n}$ is at most $M \cdot 2^{-2 n} \varepsilon^{2+2 r}$.

Together with (14) this implies that for some new constant $M^{\prime}$, the integral of $\hat{f}$ over $U_{n}$ is bounded by

$$
M^{\prime} 2^{(\delta-1) n} \varepsilon^{1+2 r-\delta}
$$

Summing over $n$, and using (15) we find that the estimate (16) holds. Lemma 7.3 is proved.

Proposition 3.3 now follows from Lemma 7.3 and Corollary 7.2

We complete this section with a proof of the statement promised in the introduction.

Proposition 7.4. For almost all flat surfaces $S$ from any stratum $\mathcal{H}(\alpha)$ of Abelian differentials one cannot find on $S$ a pair of parallel saddle connections of different lengths.

Proof. As coordinates in the stratum me may locally choose a domain in the relative cohomology space $H^{1}\left(S,\left\{P_{1}, \ldots, P_{k}\right\} ; \mathbb{C}\right)$. Let $c_{1}, c_{2}, \ldots, c_{n}$ be a basis of relative cycles in $H^{1}\left(S,\left\{P_{1}, \ldots, P_{k}\right\} ; \mathbb{Z}\right)$ The relative periods

$$
A_{i}+\sqrt{-1} \cdot B_{i}=\int_{c_{i}} \omega, \quad i=1, \ldots, n
$$

serve as the local coordinates in $\mathcal{H}(\alpha)$.

Suppose that we have two parallel saddle connections of different lengths on a flat surface $S$. They give us a pair of integer (relative) cycles

$$
s_{1}, s_{2} \in H^{1}\left(S,\left\{P_{1}, \ldots, P_{k}\right\} ; \mathbb{Z}\right) .
$$

(We don't care, whether they are both loops, or they both are segments, or one of them is a segment, and another - a loop, the argument works for any combination.) By assumption they are not homologous, so we may assume that $s_{1} \neq \pm s_{2}$. Since the cycles $s_{1}, s_{2}$ are represented by simple connected curves, they are primitive (i.e., neither of them can be represented as an integer multiple of another integer cycle). Hence, they are not collinear

$$
s_{1} \neq \lambda \cdot s_{2}
$$

even with a real $\lambda \in \mathbb{R}$.

Let $s_{1}=\sum_{i=1}^{n} k_{i} \cdot c_{i}$, where $k_{i} \in \mathbb{Z} ; s_{2}=\sum_{i=1}^{n} l_{i} \cdot c_{i}$, where $l_{i} \in \mathbb{Z}$. Since the cycles are not collinear, these two linear combinations of the basic cycles are linearly independent over reals. This means, that the rational function $f\left(x_{1}, \ldots, x_{n}\right)$ of real variables $x_{1}, \ldots, x_{n}$ 
is nonconstant.

$$
f\left(x_{1}, \ldots, x_{n}\right)=\frac{\sum_{i=1}^{n} k_{i} \cdot x_{i}}{\sum_{i=1}^{n} l_{i} \cdot x_{i}}
$$

The fact, that the two saddle connections are parallel, means, that

$$
\int_{s_{1}} \omega=\lambda \cdot \int_{s_{2}} \omega, \quad \text { with some real } \lambda \neq 0
$$

We have

$$
\int_{s_{1}} \omega=\sum_{i=1}^{n} k_{i} \cdot\left(A_{i}+\sqrt{-1} \cdot B_{i}\right) \quad \int_{s_{2}} \omega=\sum_{i=1}^{n} l_{i} \cdot\left(A_{i}+\sqrt{-1} \cdot B_{i}\right)
$$

This implies that

$$
f\left(A_{1}, \ldots, A_{n}\right)=f\left(B_{1}, \ldots, B_{n}\right),
$$

which is an algebraic condition on our coordinates $A_{1}, \ldots, A_{n}, B_{1}, \ldots, B_{n}$. (To be absolutely rigorous we have to avoid the set of measure zero defined by the algebraic condition $\sum l_{i} \cdot A_{i}=0$ or by $\sum l_{i} \cdot B_{i}=0$.) Thus, the set, satisfying this condition, has measure zero. Taking a union over the countable collection of possible conditions (countable, because we have to consider all possible pairs of integer vectors $\left.\left(k_{1}, \ldots, k_{n}\right),\left(l_{1}, \ldots, l_{n}\right)\right)$ we still get a set of measure zero.

\section{Part 1. Saddle Connections Joining Distinct Zeroes}

In this part we describe the possible configurations of saddle connections joining distinct zeroes and compute the constants in the corresponding asymptotics.

To separate the basic construction and numerous details we start with the easiest case. In the first section of this part we assume that the saddle connection joining a pair of zeroes $P_{i}, P_{j}$ has multiplicity one, i.e., there are no other saddle connections in the same direction joining the same pair of zeroes. In this section we also assume that the translation surface under consideration belongs to a connected stratum.

In the second section of this part we consider the problem in full generality for the translation surfaces from connected strata. In particular we give the explicit general formula for the surfaces from the principal stratum.

In the last section of this part we treat the surfaces from the strata which are not connected.

\section{Saddle Connections of Multiplicity One. Connected Strata}

8.1. Breaking up a Zero. Suppose we are given a flat surface $S^{\prime}$ defined by $\omega^{\prime}$, a zero $w$ of order $m \geq 2$, a pair of positive integers $m_{1}, m_{2}$, such that $m=m_{1}+m_{2}$, and a vector $\gamma \in \mathbb{R}^{2}$ of length $2 \delta \leq \varepsilon$. Further suppose that $S^{\prime}$ does not have any saddle connection or closed geodesic of length smaller than $2 \varepsilon$. Let $w, z_{1}, \ldots, z_{l}$ be the set of all zeroes of the flat structure on $S^{\prime}$. If $w$ is the only zero of $\omega^{\prime}$ choose a basis of cycles for the relative homology group $H_{1}\left(S^{\prime},\{w\} ; \mathbb{Z}\right)$ all of which miss $w$. If there are other zeroes, we may choose a basis of cycles in the relative homology group $H_{1}\left(S^{\prime},\left\{w, z_{1}, \ldots, z_{l}\right\} ; \mathbb{Z}\right)$ such that exactly one curve $\beta_{1}$ contains $w$ and $\beta_{1}$ is not closed; that is, $w$ is an endpoint of $\beta_{1}$. 
We take a disc of radius $\varepsilon$ about $w$ that misses all other zeroes. We may break up the zero $w$ of order $m$ on $S^{\prime}$ into two zeroes $z^{\prime}, z^{\prime \prime}$ of orders $m_{1}$ and $m_{2}$ correspondingly with a vector $\gamma$ joining them constructing a flat surface $S$. We can describe this breakup as a Whitehead move on the foliation in direction $\gamma$. We do this by forming $2 m+2$ half discs of radius $\varepsilon$. Along the real axis of two of them we mark points at distance $\delta$ from the origin. These two discs are glued together along the corresponding segment of length $2 \delta$ leaving a pair of free segments of length $\varepsilon-\delta$ on each. On each of the remaining discs we mark a point at distance $\delta$ from the origin leaving segments of length $\varepsilon+\delta$ and $\varepsilon-\delta$. We now glue the segments isometrically to each other in a circular fashion, see Figure 3
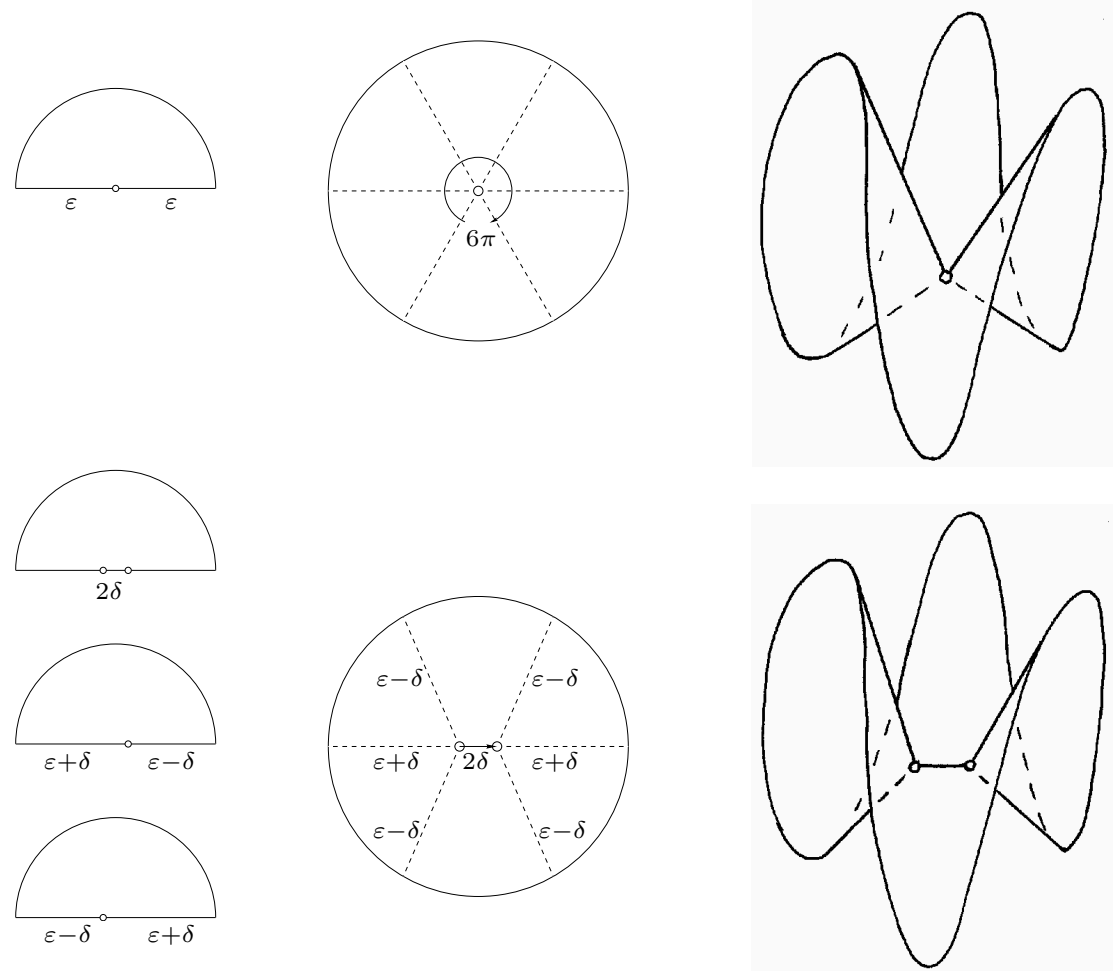

Figure 3. Breaking up a zero of degree 2 into two simple zeroes.

Note that the surgery is local: we do not change the flat metric outside of the neighborhood of the zero.

If $m_{1} \neq m_{2}$, the number of ways of effecting the breakup is $2 m+2$. However, we have $\overrightarrow{z^{\prime} z^{\prime \prime}}=\gamma$ for only half of the resulting surfaces, i.e. for $m+1$ ones; for another $m+1$ surfaces we have $\overrightarrow{z^{\prime \prime} z^{\prime}}=\gamma$. If $m_{1}=m_{2}=m / 2$ the number of the resulting surfaces is $m+1$. For every such surface there are two ways to assign "names" $z^{\prime}, z^{\prime \prime}$ to the newborn zeroes of order $m / 2$. This doubles the number of resulting surfaces with "named" zeroes. However, we again have only $m+1$ surfaces with $\overrightarrow{z^{\prime} z^{\prime \prime}}=\gamma$; for another $m+1$ ones we have $\overline{z^{\prime \prime} z^{\prime}}=\gamma$. We again choose only those $m+1$ surfaces for which we have $\overrightarrow{z^{\prime} z^{\prime \prime}}=\gamma$. 
By convention we let the curve which had the endpoint at $w$ (if it was present) keep the corresponding endpoint at $z^{\prime}$ during the deformation. The fact that this construction was local means that except for the curve $\beta_{1}$ (if it exists) the holonomy is preserved along the homology basis of $S^{\prime}$. The holonomy of $\beta_{1}$ is changed by $-\gamma / 2$. Furthermore every saddle connection other than $\overrightarrow{z^{\prime} z^{\prime \prime}}$ has length at least $\varepsilon$.

We denote the assignment by

$$
\left(S^{\prime}, \gamma, m\right) \rightarrow\left(S, m_{1}, m_{2}\right)
$$

We denote by $\mathcal{H}_{1}(\alpha)$ the stratum which contains the resulting flat surface $S$. By construction the partition $\alpha$ is obtained from the partition $\alpha^{\prime}$ by replacing the entry $m$ by two entries $m_{1}$ and $m_{2}$.

Recall that all the zeroes on the surface $S^{\prime}$ are "named". For all the zeroes on the new surface $S$ different from the zero which was just broken we keep the same "names" on $S$ as their initial "names" on $S^{\prime}$.

8.2. Collapsing a Pair of Zeroes, Principal Boundary. Now conversely, suppose we have a surface $S \in \mathcal{H}_{1}(\alpha)$, a saddle connection of length $2 \delta \leq \varepsilon$ joining distinct zeroes $w_{1}, w_{2}$ of orders $m_{1}$ and $m_{2}$ with holonomy $\gamma$, and no other saddle connection of length smaller than $3 \varepsilon$. Let $z_{1}, \ldots, z_{l}$ be the other zeroes. Choose a basis of cycles in the relative homology group $H_{1}\left(S,\left\{w_{1}, w_{2}, z_{1}, \ldots, z_{l}\right\} ; \mathbb{Z}\right)$. This basis can be represented by a collection of curves $\beta_{i}$ on the surface $S$. One of these curves, $\beta_{0}$ is the saddle connection joining $w_{1}$ and $w_{2}$. If these are the only zeroes, then we can choose a basis of cycles in such way that all other cycles are closed and miss $w_{1}$ and $w_{2}$. If there are other zeroes, then a single curve $\beta_{1}$ intersects one of the $w_{i}$, say $w_{1}$. The curve $\beta_{1}$ is not closed; it has $w_{1}$ as an endpoint.

One can now exactly reverse the breaking up procedure to collapse the saddle connection of length $2 \delta$ to a zero $w$ of order $m=m_{1}+m_{2}$ to construct a flat surface $S^{\prime}$. Namely, we can describe a neighborhood of the saddle connection as a union of $2 m+2$ half discs of radius $\varepsilon$ glued along pieces of their boundary. Two of the half discs are glued along segments of length $2 \delta$ to form the saddle connection. This leaves a pair of segments of length $\varepsilon-\delta$ on each side. The other discs have marked segments of length $\varepsilon-\delta$ and $\varepsilon+\delta$ which are glued isometrically to form the neighborhood. We deform $S$ in the neighborhood by taking $2 m+2$ half discs of radius $\varepsilon$ and gluing them cyclically along segments of length $\varepsilon$. The saddle connection $\beta_{0}$ is collapsed to a point which becomes a zero $w$ of order $m$. We may perform this deformation while keeping the flat structure in the complement of the neighborhood fixed, see section 8.1 and Figures 3 and 4 .

If $\beta_{1}$ exists then we can replace $\beta_{1}$ with a curve joined to $w$. The fact that the deformation is local means that the holonomy is fixed along the entire basis other than $\beta_{0}$ and $\beta_{1}$. The holonomy of $\beta_{1}$ is changed by adding $\gamma / 2$. Furthermore, every saddle connection on $S^{\prime}$ has length at least $2 \varepsilon$. All named zeroes on $S$ not affected by the collapse are given the same names on $S^{\prime}$. We may think of this surgery as of a Whitehead move on the foliation in direction $\gamma$. The resulting surface $S^{\prime}$ belongs to a stratum $\mathcal{H}_{1}\left(\alpha^{\prime}\right)$. By construction the partition $\alpha^{\prime}$ is obtained from the partition $\alpha$ by replacing the pair of entries $m_{1}, m_{2}$ by the entry $m$. The stratum $\mathcal{H}_{1}\left(\alpha^{\prime}\right)$ is in the closure of $\mathcal{H}_{1}(\alpha)$ inside the moduli space of all flat structures on the surfaces of genus $g$. We say that $\mathcal{H}_{1}\left(\alpha^{\prime}\right)$ is the principal boundary of $\mathcal{H}_{1}(\alpha)$ corresponding to this configuration. 
Now choose a simply connected subset of $\mathcal{H}_{1}\left(\alpha^{\prime}\right)$ of full volume and remove the set of flat surfaces with a saddle connection or closed geodesic of length at most $2 \varepsilon$. Call the resulting set $\mathcal{F}^{\prime} \subset \mathcal{H}_{1}\left(\alpha^{\prime}\right)$. By Lemma 7.1

$$
\operatorname{Vol}\left(\mathcal{H}_{1}\left(\alpha^{\prime}\right)-\mathcal{F}^{\prime}\right)=O\left(\varepsilon^{2}\right)
$$

Choose a homology basis that is valid for all $S^{\prime} \in \mathcal{F}^{\prime}$. Thus distinct $S_{1}^{\prime}$, $S_{2}^{\prime}$ have different holonomy on some basis element. As in Section 7 for $\kappa=\varepsilon$ or $3 \varepsilon$ let $\mathcal{H}_{1}^{\varepsilon, \kappa}(\alpha, \mathcal{C}) \subset \mathcal{H}_{1}(\alpha)$ be the set of flat surfaces with a saddle connection of length at most $\varepsilon$ joining the named zeroes and no other saddle connections of length smaller than $\kappa$; the configuration $\mathcal{C}$ corresponds to a single saddle connection. We have

Lemma 8.1. For $\gamma$ a vector in $\mathbb{R}^{2}$ of length at most $\varepsilon$, except for a set of $S^{\prime} \in \mathcal{F}^{\prime}$ of volume 0 , there are precisely $m+1$ surfaces in $\mathcal{H}_{1}^{\varepsilon, \varepsilon}(\alpha, \mathcal{C})$ that are the result of the assignment $\left(S^{\prime}, \gamma, m\right) \rightarrow\left(S, m_{1}, m_{2}\right)$. Moreover, every surface in $\mathcal{H}_{1}^{\varepsilon, 3 \varepsilon}(\alpha, \mathcal{C})$ is the result of such an assignment.

Proof. We have addressed every issue except the statement that there are precisely $m+1$ surfaces obtained as the result of the assignment. Suppose that for fixed $S^{\prime} \in \mathcal{F}^{\prime} \subset \mathcal{H}_{1}\left(\alpha^{\prime}\right)$ two of the $m+1$ surfaces $S$ built from $S^{\prime}$ are isomorphic. Since by construction each of these surfaces has a single short saddle connection, the isomorphism sends the newborn saddle connection on one surface to the newborn saddle connection on the other surface. Hence, it sends the corresponding "disc" on one surface to the corresponding "disc" on the other surface, see Figure 4 Hence, it is an isomorphism of the complements of the "discs", which implies that it induces an automorphism of the surface $S^{\prime}$. It is sufficient to note that the set of flat surfaces, that have automorphisms, has measure 0 .

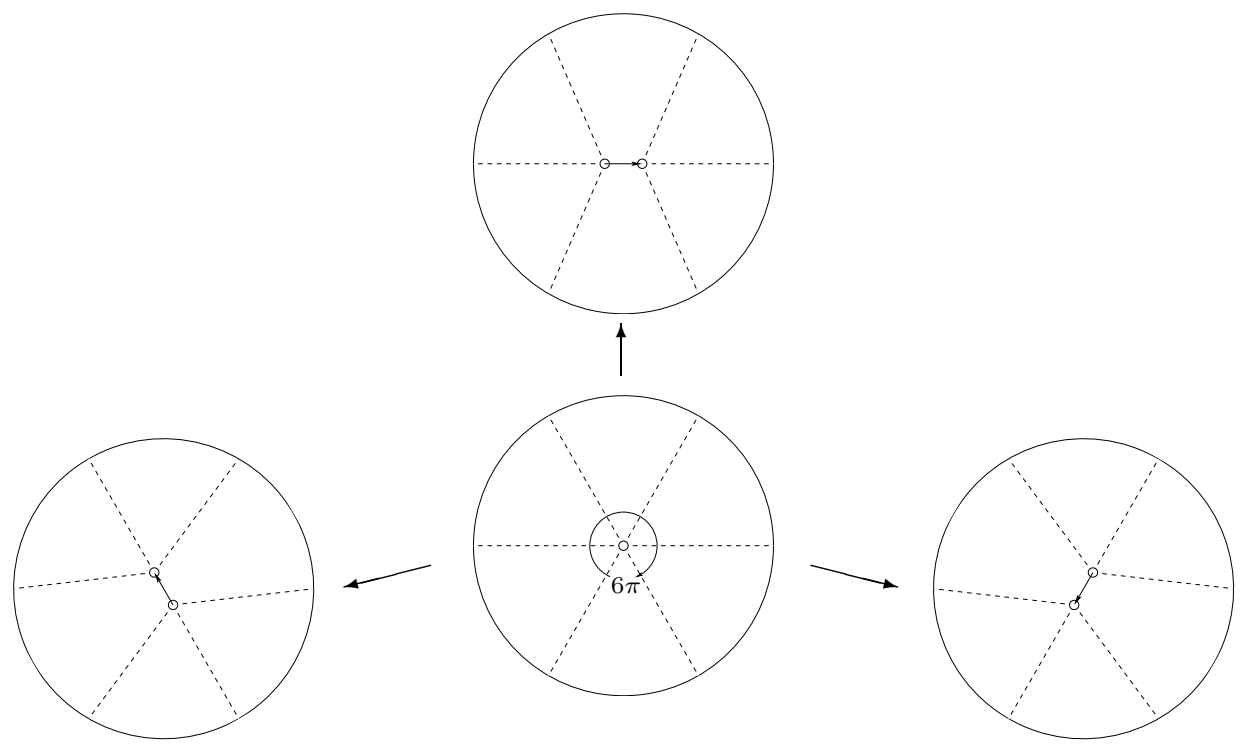

Figure 4. The cone angle corresponding to a zero of order $m=2$ is equal to $(m+1) \cdot 2 \pi=6 \pi$. Thus we have $(m+1)=3$ different ways of breaking up a zero of order 2 in a direction $\vec{\gamma}$. In this way (generically) we get $m=3$ different flat surfaces 
Remark 8.2. Note that the cohomology class $[\omega] \in H^{1}\left(S,\left\{w, z_{1}, \ldots, z_{l}\right\} ; \mathbb{C}\right)$ together with $\overrightarrow{z^{\prime} z^{\prime \prime}}=\gamma$ determines an element of the relative cohomology group $[\omega] \in H^{1}\left(S,\left\{z^{\prime}, z^{\prime \prime}, z_{1}, \ldots, z_{l}\right\} ; \mathbb{C}\right)$. The lemma above claims, actually, that the local mapping $\mathcal{H}_{1}^{\varepsilon, 3 \varepsilon}(\alpha, \mathcal{C}) \rightarrow \mathcal{F}^{\prime} \times B(\varepsilon)$ is a ramified covering of order $m+1$ almost everywhere and that $d \nu(S)=d \nu\left(S^{\prime}\right) d \gamma$.

8.3. Computing the Siegel-Veech Constants. In this section we derive formulae for the constant $c$ in quadratic asymptotics of the number of saddle connections joining a zero of order $m_{1}$ to a zero of order $m_{2}$ in multiplicity one. According to Proposition 3.3 this means that we have to find the asymptotics of $\operatorname{Vol}\left(\mathcal{H}_{1}^{\varepsilon, \varepsilon}(\alpha, \mathcal{C})\right)$, where the configuration $\mathcal{C}$ corresponds to a single saddle connection. Recall also that we are assuming that the zeroes are named. Since we may have numerous zeroes of orders $m_{1}$ and $m_{2}$ we, actually, have two different counting problems:

Problem 1. Count the constant in the quadratic asymptotics for the number of saddle connections joining a fixed zero $z_{1}$ of order $m_{1}$ to a fixed zero $z_{2}$ of order $m_{2}$. (When $m_{1}=m_{2}$ we require that $z_{1} \neq z_{2}$.)

Problem 2. Count the constant in the quadratic asymptotics for the number of saddle connections joining some zero of order $m_{1}$ to some other zero of order $m_{2}$. (This is, in fact, equivalent to counting the saddle connections joining unnumbered zeroes.)

Let us start by assuming that $\mathcal{H}_{1}(\alpha)$ is connected. The case of strata that are not connected will be postponed until the end of the section on higher multiplicity.

In the computations to follow we will obscure the distinction between $\gamma$ as a saddle connection in the configuration and its holonomy. Thus we will use $\gamma$ as a vector in the disc $B(\varepsilon)$ and as a variable of integration.

Again let $n=\operatorname{dim}_{\mathbb{R}} \mathcal{H}(\alpha), n^{\prime}=\operatorname{dim}_{\mathbb{R}} \mathcal{H}\left(\alpha^{\prime}\right)$. Then $n^{\prime}=n-2$. Recall that $d \nu^{\prime}\left(S^{\prime}\right)$ is the natural measure on $\mathcal{H}\left(\alpha^{\prime}\right)$. For $S^{\prime} \in C\left(\mathcal{F}^{\prime}\right)$ we set $S^{\prime}=r S^{\prime \prime}$ where $\operatorname{area}\left(S^{\prime \prime}\right)=1$ so area $\left(S^{\prime}\right)=r^{2}$. Then by Remark 8.2

$$
d \nu(S)=d \nu^{\prime}\left(S^{\prime}\right) d \gamma=r^{n^{\prime}-1} d r d \operatorname{vol}^{\prime}\left(S^{\prime \prime}\right) d \gamma
$$

By Lemma 7.1

$$
\operatorname{Vol}\left(\mathcal{H}_{1}^{\varepsilon, \varepsilon}(\alpha, \mathcal{C})-\mathcal{H}_{1}^{\varepsilon, 3 \varepsilon}(\alpha, \mathcal{C})\right)=O\left(\varepsilon^{4}\right)
$$

so by Lemma 8.1

$$
\begin{aligned}
\nu\left(C\left(\mathcal{H}_{1}^{\varepsilon, \varepsilon}(\alpha, \mathcal{C})\right)\right) & =\left(m_{1}+m_{2}+1\right) \operatorname{Vol}\left(\mathcal{F}^{\prime}\right) \int_{0}^{1} r^{n^{\prime}-1} \int_{B(\varepsilon r)} d \gamma d r+O\left(\varepsilon^{4}\right) \\
& =\pi \varepsilon^{2}\left(m_{1}+m_{2}+1\right) \operatorname{Vol}\left(\mathcal{F}^{\prime}\right) \int_{0}^{1} r^{n^{\prime}+1} d r+O\left(\varepsilon^{4}\right) \\
& =\frac{\pi \varepsilon^{2}}{n^{\prime}+2}\left(m_{1}+m_{2}+1\right) \operatorname{Vol}\left(\mathcal{F}^{\prime}\right)+O\left(\varepsilon^{4}\right) .
\end{aligned}
$$

Since $n=n^{\prime}+2$ we have

$$
\operatorname{Vol}\left(\mathcal{H}_{1}^{\varepsilon, \varepsilon}(\alpha, \mathcal{C})\right)=n \nu\left(C\left(\mathcal{H}_{1}^{\varepsilon, \varepsilon}(\alpha)\right)\right)=\left(m_{1}+m_{2}+1\right) \pi \varepsilon^{2} \operatorname{Vol}\left(\mathcal{F}^{\prime}\right)+O\left(\varepsilon^{4}\right)
$$


Thus, the constant from the Problem 1 has the following form:

$$
\begin{gathered}
c=\lim _{\varepsilon \rightarrow 0} \frac{\operatorname{Vol}\left(\mathcal{H}_{1}^{\varepsilon, \varepsilon}(\alpha, \mathcal{C})\right)}{\pi \varepsilon^{2} \operatorname{Vol}\left(\mathcal{H}_{1}(\alpha)\right)}=\lim _{\varepsilon \rightarrow 0} \frac{\left.\left(m_{1}+m_{2}+1\right) \operatorname{Vol}\left(\mathcal{F}^{\prime}\right)\right)}{\operatorname{Vol}\left(\mathcal{H}_{1}(\alpha)\right)}= \\
=\frac{\left(m_{1}+m_{2}+1\right) \operatorname{Vol}\left(\mathcal{H}_{1}\left(\alpha^{\prime}\right)\right)}{\operatorname{Vol}\left(\mathcal{H}_{1}(\alpha)\right)} .
\end{gathered}
$$

Note that having solved the Problem 1 we get a solution of Problem 2 by an elementary combinatorial calculation. Let $o\left(m_{i}\right)$ be the number of zeroes of order $m_{i}$ in the stratum $\mathcal{H}(\alpha)$. If $m_{1} \neq m_{2}$, then there are $o\left(m_{1}\right)$ ways of choosing the zero of order $m_{1}$ and $o\left(m_{2}\right)$ ways of choosing the zero of order $m_{2}$. Thus we get an additional factor $o\left(m_{1}\right) \cdot o\left(m_{2}\right)$ in comparison with Problem 1.

Formula 8.1. The constant in any connected stratum $\mathcal{H}_{1}(\alpha)$ for the number of saddle connections of multiplicity one joining two zeroes of orders $m_{1} \neq m_{2}$ is equal to

$$
c=o\left(m_{1}\right) o\left(m_{2}\right) \cdot\left(m_{1}+m_{2}+1\right) \cdot \frac{\operatorname{Vol}\left(\mathcal{H}_{1}\left(\alpha^{\prime}\right)\right)}{\operatorname{Vol}\left(\mathcal{H}_{1}(\alpha)\right)} \quad \text { when } m_{1} \neq m_{2}
$$

If $m_{1}=m_{2}$, then there are $o\left(m_{1}\right)\left(o\left(m_{1}\right)-1\right) / 2$ ways of choosing an unordered pair of zeroes of order $m_{1}$. Having chosen an unordered pair, we choose, which of two zeroes would called be $z_{1}$, and which one would be $z_{2}$ in arbitrary way: the number of saddle connections is obviously symmetric with respect to interchange of the names $z_{1}, z_{2}$. Thus, when $m_{1}=m_{2}$ the answer to Problem 2 is given as the answer for Problem 1 multiplied by an additional factor $o\left(m_{1}\right)\left(o\left(m_{1}\right)-1\right) / 2$.

Formula 8.2. The constant for the number of saddle connections of multiplicity one joining two distinct zeroes of the same orders $m_{1}$ is equal to

$$
c=\frac{o\left(m_{1}\right)\left(o\left(m_{1}\right)-1\right)}{2} \cdot\left(2 m_{1}+1\right) \cdot \frac{\operatorname{Vol}\left(\mathcal{H}_{1}\left(\alpha^{\prime}\right)\right)}{\operatorname{Vol}\left(\mathcal{H}_{1}(\alpha)\right)} \quad \text { when } m_{1}=m_{2}
$$

Note that we may view the entire combinatorial calculation in terms of the naming of the zeroes. We can assume that on $S^{\prime}$ we have broken up the zero of order $m=m_{1}+m_{2}$ at the first "named" zero of order $m$. The remaining zeroes are then given the same names on $S$. Other zeroes of orders $m_{1}, m_{2}$ already have names on $S$. There are $o\left(m_{1}\right) o\left(m_{2}\right)$ ways of adding additional names to zeroes of orders $m_{1}, m_{2}$ if $m_{1} \neq m_{2}$ and $o\left(m_{1}\right)\left(o\left(m_{1}\right)-1\right)$ if $m_{1}=m_{2}$.

Note that in the consideration above the stratum $\mathcal{H}_{1}\left(\alpha^{\prime}\right)$ might be nonconnected. In this case $\operatorname{Vol}\left(\mathcal{H}_{1}\left(\alpha^{\prime}\right)\right)$ is the sum of the volumes of connected components. For example if we start with the stratum $\mathcal{H}_{1}(5,3)$, then $\mathcal{H}_{1}\left(\alpha^{\prime}\right)$ has three connected components $\mathcal{H}_{1}^{\text {hyp }}(8), \mathcal{H}_{1}^{\text {even }}(8)$ and $\mathcal{H}_{1}^{\text {odd }}(8)$, and the sum of the volumes of these must be in the numerator.

8.4. Examples: Constants for Connected Strata in Genus 3. As an illustration of the formulae above we now give the explicit values for these constants in a number of cases.

In Table 1 we present the normalized volumes $\frac{1}{\pi^{2 g}} \operatorname{Vol}\left(\mathcal{H}_{1}(\alpha)\right)$ of the strata with numbered zeroes, see [EOk], see also some values in [Z]. These values will be used for computations in all the examples. 
TABLE 1. Normalized volumes $\frac{1}{\pi^{2 g}} \operatorname{Vol}\left(\mathcal{H}_{1}(\alpha)\right)$ of the strata in small genera

Genera $g=1,2$
\begin{tabular}{|c|c|c|}
\hline $\mathcal{H}_{1}(0)$ & $\mathcal{H}_{1}(2)$ & $\mathcal{H}_{1}(1,1)$ \\
\hline$\frac{1}{3}$ & $\frac{1}{120}$ & $\frac{1}{135}$ \\
\hline
\end{tabular}

\begin{tabular}{|c|c|c|c|c|c|c|}
\hline $\mathcal{H}_{1}^{\text {hyp }}(4)$ & $\mathcal{H}_{1}^{\text {odd }}(4)$ & $\mathcal{H}_{1}(3,1)$ & $\mathcal{H}_{1}^{\text {hyp }}(2,2)$ & $\mathcal{H}_{1}^{\text {odd }}(2,2)$ & $\mathcal{H}_{1}(2,1,1)$ & $\mathcal{H}_{1}(1,1,1,1)$ \\
\hline$\frac{1}{6720}$ & $\frac{1}{2430}$ & $\frac{16}{42525}$ & $\frac{1}{9450}$ & $\frac{1}{4320}$ & $\frac{1}{3780}$ & $\frac{1}{4860}$ \\
\hline
\end{tabular}

\begin{tabular}{|c|c|c|c|}
\hline \multicolumn{4}{|c|}{ Genus $g=4$} \\
\hline $\mathcal{H}_{1}^{\text {hyp }}(6)$ & $\mathcal{H}_{1}^{\text {odd }}(6)$ & $\mathcal{H}_{1}^{\text {even }}(6)$ & $\mathcal{H}_{1}(5,1)$ \\
\hline$\frac{1}{580608}$ & $\frac{1}{37800}$ & $\frac{32}{1913625}$ & $\frac{1}{36750}$ \\
\hline \hline $\mathcal{H}_{1}^{\text {odd }}(4,2)$ & $\mathcal{H}_{1}^{\text {even }}(4,2)$ & $\mathcal{H}_{1}(4,1,1)$ & $\mathcal{H}_{1}^{\text {nonhyp }}(3,3)$ \\
\hline$\frac{1}{79380}$ & $\frac{1}{107520}$ & $\frac{11}{653184}$ & $\frac{1}{51030}$ \\
\hline $\mathcal{H}_{1}^{\text {hyp }}(3,3)$ & $\mathcal{H}_{1}(3,2,1)$ & $\mathcal{H}_{1}(3,1,1,1)$ & $\mathcal{H}_{1}^{\text {odd }}(2,2,2)$ \\
\hline$\frac{1}{992250}$ & $\frac{1}{70875}$ & $\frac{62}{5740875}$ & $\frac{31}{4354560}$ \\
\hline \hline $\mathcal{H}_{1}^{\text {even }}(2,2,2)$ & $\mathcal{H}_{1}(2,2,1,1)$ & $\mathcal{H}_{1}(2,1,1,1,1)$ & $\mathcal{H}_{1}(1, \ldots, 1)$ \\
\hline$\frac{37}{6804000}$ & $\frac{131}{13608000}$ & $\frac{1}{136080}$ & $\frac{377}{67359600}$ \\
\hline
\end{tabular}

Both strata in genus $g=2$ are hyperelliptic; they will be treated later on in section 10 In genus $g=3$ there are three connected strata: $\mathcal{H}_{1}(3,1), \mathcal{H}_{1}(2,1,1)$, and $\mathcal{H}_{1}(1,1,1,1)$.

Example 8.3. Stratum $\mathcal{H}_{\mathbf{1}}(\mathbf{3}, \mathbf{1})$. After collapsing zeroes we obtain a flat surface $S^{\prime} \in \mathcal{H}_{1}(4)$, where both components $\mathcal{H}_{1}^{\text {hyp }}(4)$ and $\mathcal{H}_{1}^{\text {odd }}(4)$ occur. We have $m_{1}=$ $3, m_{2}=1$, thus the combinatorial factor equals $o\left(m_{1}\right) o\left(m_{2}\right)\left(m_{1}+m_{2}+1\right)=5$. We get

$$
c=5 \cdot \frac{\operatorname{Vol}\left(\mathcal{H}_{1}(4)\right)}{\operatorname{Vol}\left(\mathcal{H}_{1}(3,1)\right)}=5 \cdot \frac{\operatorname{Vol}\left(\mathcal{H}_{1}^{\text {hyp }}(4)\right)+\operatorname{Vol}\left(\mathcal{H}_{1}^{\text {odd }}(4)\right)}{\operatorname{Vol}\left(\mathcal{H}_{1}(3,1)\right)}=\frac{7625}{1024} \approx 7.45
$$

Example 8.4. Stratum $\mathcal{H}_{\mathbf{1}}(\mathbf{2}, \mathbf{1}, \mathbf{1})$. There are two cases here in multiplicity 1. The first case is a saddle connection joining the zero of degree 2 with any of the two zeroes of degree 1 . Collapsing the zeroes we obtain a surface $S^{\prime} \in \mathcal{H}_{1}(3,1)$. For 
$m_{1}=2, m_{2}=1, o\left(m_{1}\right)=1, o\left(m_{2}\right)=2$. Thus the combinatorial constant equals 8 . We get

$$
c=8 \cdot \frac{\operatorname{Vol}\left(\mathcal{H}_{1}(3,1)\right)}{\operatorname{Vol}\left(\mathcal{H}_{1}(2,1,1)\right)}=\frac{512}{45} \approx 11.4
$$

The second case consists of collapsing the pair of simple zeroes to a zero of order 2. We obtain a surface $S^{\prime} \in \mathcal{H}_{1}(2,2)$. Any surface in any component of $\mathcal{H}_{1}(2,2)$ can be found by collapsing a pair of simple zeroes in this manner. Here $m_{1}=m_{2}=1$ and $o\left(m_{1}\right)=2$ so the combinatorial constant is 3 . We get

$$
c=3 \cdot \frac{\operatorname{Vol}\left(\mathcal{H}_{1}(2,2)\right)}{\operatorname{Vol}\left(\mathcal{H}_{1}(2,1,1)\right)}=3 \cdot \frac{\operatorname{Vol}\left(\mathcal{H}_{1}^{\text {hyp }}(2,2)\right)+\operatorname{Vol}\left(\mathcal{H}_{1}^{\text {odd }}(2,2)\right)}{\operatorname{Vol}\left(\mathcal{H}_{1}(2,1,1)\right)}=\frac{153}{40} \approx 3.83
$$

8.5. Constants for the Principal Stratum $\mathcal{H}_{1}(1, \ldots, 1)$. The surface of genus $g$ has $2 g-2$ simple zeroes; $o(1)=2 g-2$. The corresponding surface $S^{\prime} \in$ $\mathcal{H}_{1}(2,1, \ldots, 1)$ has $2 g-4$ simple zeroes. The factor $o\left(m_{1}\right)\left(o\left(m_{1}\right)-1\right) / 2$ gives $(g-1)(2 g-3)$, and the factor $\left(2 m_{1}+1\right)$ gives 3. Applying formula (18)) we get

Formula 8.3. The constant for the number of saddle connections of multiplicity one joining two distinct zeroes is equal to

$$
c=3(g-1)(2 g-3) \cdot \frac{\operatorname{Vol}\left(\mathcal{H}_{1}(2, \overbrace{1, \ldots, 1}^{2 g-4})\right)}{\operatorname{Vol}\left(\mathcal{H}_{1}(\underbrace{1, \ldots, 1}_{2 g-2})\right)}
$$

In the table below we present some values of the constant in this case.

TABLE 2. Principal stratum $\mathcal{H}_{1}(1, \ldots, 1)$; values of the constants for saddle connections of multiplicity one joining distinct zeroes

\begin{tabular}{|l|c|c|c|c|c|c|c|}
\hline & $g=2$ & $g=3$ & $g=4$ & $g=5$ & $g=6$ & $g=7$ & $g=8$ \\
\hline$c=$ & $\frac{27}{8}$ & $\frac{162}{7}$ & $\frac{22275}{377}$ & $\frac{2594700}{23357}$ & $\frac{2954056635}{16493303}$ & $\frac{13229971542}{50280671}$ & $\frac{14740938123723}{40593663941}$ \\
\hline$c \approx$ & 3.375 & 23.14 & 59.08 & 111.1 & 179.1 & 263.1 & 363.1 \\
\hline
\end{tabular}

\section{Multiple Homologous Saddle Connections. Connected Strata}

9.1. Principal Boundary. Consider a surface $S \in \mathcal{H}_{1}(\alpha)$ with a fixed pair of zeroes $z_{1}$ and $z_{2}$ of orders $m_{1}$ and $m_{2}$ correspondingly. Suppose that we have a configuration $\mathcal{C}$ (see Section 3 ) of precisely $p$ homologous saddle connections $\gamma_{1}, \ldots, \gamma_{p}$ joining $z_{1}$ to $z_{2}$ of length at most $\varepsilon$. Assume there are no other saddle connections shorter than $3 \varepsilon$. A pair $\gamma_{i}$ and $\gamma_{i+1}$ bounds a surface $S_{i}$. The surfaces $S_{i}$ and $S_{i+1}$ share the saddle connection $\gamma_{i+1}$. By convention the cyclic order of $\gamma_{i}$ at $z_{1}$ is clockwise in the orientation defined by the flat structure. The angle between $\gamma_{i}$ and $\gamma_{i+1}$ at $z_{1}$ is $2 \pi\left(a_{i}^{\prime}+1\right)$; the angle between $\gamma_{i}$ and $\gamma_{i+1}$ at $z_{2}$ be $2 \pi\left(a_{i}^{\prime \prime}+1\right)$.

Cut the surface $S$ along all $\gamma_{i}, i=1, \ldots, p$. Gluing together the two sides $\gamma_{i}$ and $\gamma_{i+1}$ of the boundary of $S_{i}$ we get a flat surface with two distinguished zeroes 
$z_{i}^{\prime}, z_{i}^{\prime \prime}$ of orders $a_{i}^{\prime}$ and $a_{i}^{\prime \prime}$ correspondingly joined by a saddle connection, $\overrightarrow{z_{i}^{\prime} z_{i}^{\prime \prime}}=\gamma$. When one of $a_{i}^{\prime}, a_{i}^{\prime \prime}$ is equal to zero the corresponding point is just a marked point. If $a_{i}^{\prime}=a_{i}^{\prime \prime}=0$, then both points are marked points.

By construction any resulting flat surface $S_{i}$ has a saddle connection $\gamma_{i}$ shorter than $\varepsilon$ joining the pair of zeroes and does not have any other saddle connections homologous to it. Every other saddle connection is longer than $3 \varepsilon$. This implies that contracting the saddle connection $\gamma_{i}$ to a point, see section 8.2 we get a flat surface $S_{i}^{\prime}$ with a distinguished zero (marked point) $w_{i}$ of order $a_{i}=a_{i}^{\prime}+a_{i}^{\prime \prime}$ and no saddle connection shorter than $2 \varepsilon$.

Let $S_{i}^{\prime} \in \mathcal{H}\left(\alpha_{i}^{\prime}\right)$. We say that $\sqcup_{i=1}^{p} \mathcal{H}\left(\alpha_{i}^{\prime}\right)$ is the principal boundary of this configuration. Note that if $a_{i}=0$ we mark corresponding point on $S_{i}^{\prime}$, so collection $\alpha_{i}^{\prime}$ contains 0 in this case. We use the notation $\alpha^{\prime}=\sqcup \alpha_{i}^{\prime}$, see Convention 5

9.2. Slit Construction. We would like to reverse the above degeneration and build surfaces with multiple homologous saddle connections out of surfaces in $\mathcal{H}\left(\alpha_{i}^{\prime}\right)$. We need the following construction. Let $S^{\prime}$ be a surface with a zero of order $a \geq 0$. Suppose there are no saddle connections of length shorter than $2 \varepsilon$. Let $a^{\prime}, a^{\prime \prime} \geq 0$ such that $a^{\prime}+a^{\prime \prime}=a$. Let $\gamma$ be a vector of length at most $\varepsilon$. If $a^{\prime}, a^{\prime \prime} \neq 0$ we may break up the zero of order $a$ into zeroes of orders $a^{\prime}, a^{\prime \prime}$ with a saddle connection determining $\gamma$ joining them, see section 8.1. If $a^{\prime}=0$ take a point $z^{\prime}$ of the form $w-\gamma$ (this means along a geodesic from $w$ in direction $-\gamma$ and distance $|\gamma|$ ) and join it to $z^{\prime \prime}=w$ on $S^{\prime}$. If $a^{\prime \prime}=0$ we take a segment from $z^{\prime}=w$ to $z^{\prime \prime}=w+\gamma$ on $S^{\prime}$.

In either case we may slit the resulting surface along the saddle connection joining the two zeroes $z^{\prime}, z^{\prime \prime}$. In this way we build a surface with one boundary component consisting of two arcs, denoted $\gamma^{\prime}$ and $\gamma^{\prime \prime}$, joining the endpoints of the slit. The angles between $\gamma^{\prime}$ and $\gamma^{\prime \prime}$ at the points $z^{\prime}$ and $z^{\prime \prime}$ are $2 \pi\left(a^{\prime}+1\right)$ and $2 \pi\left(a^{\prime \prime}+1\right)$ correspondingly.

Note that the flat structure on $S^{\prime}$ fixes the choice of the orientation. By convention we give the "names" $\gamma^{\prime}$ and $\gamma^{\prime \prime}$ to the arcs in such a way that turning around $z^{\prime}$ in a clockwise direction from $\gamma^{\prime \prime}$ to $\gamma^{\prime}$ we do not leave the surface.

Conversely, consider a flat surface with a single boundary component consisting of two arcs joining a pair of vertices. The convention on the choice of the "names" $\gamma^{\prime}$ and $\gamma^{\prime \prime}$ means that as soon as we know which of two vertices is $z^{\prime}$, and which is $z^{\prime \prime}$ we can determine which of two arcs is $\gamma^{\prime}$, and which is $\gamma^{\prime \prime}$.

We describe now how to build surfaces with multiple homologous saddle connections using the slit construction.

9.3. Building Surfaces With Multiple Homologous Saddle Connections. Suppose $S^{\prime}=S_{1}^{\prime} \sqcup \ldots \sqcup S_{p}^{\prime}$ is a disconnected flat surface and on each $S_{i}^{\prime}$ there is a zero or a regular point which we think of as a marked point $w_{i}$ of order $a_{i}$. Assume no surface contains a saddle connection shorter than $2 \varepsilon$. Given pairs $a_{i}^{\prime}, a_{i}^{\prime \prime}$ with $a_{i}=a_{i}^{\prime}+a_{i}^{\prime \prime}$, and a vector $\gamma$ of length smaller than $\varepsilon$, we perform the slit construction on each surface. We obtain surfaces with one boundary component each of which consists of two arcs $\gamma_{i}^{\prime}$ and $\gamma_{i}^{\prime \prime}$. We glue $\gamma_{i}^{\prime}$ to $\gamma_{i+1}^{\prime \prime}$, calling this curve $\gamma_{i+1}$, (and $\gamma_{p}^{\prime}$ to $\gamma_{1}^{\prime \prime}$ ). This gives a closed surface $S$, a pair of zeroes $z_{1}$ and $z_{2}$ of orders $m_{1}$ and $m_{2}$, where $\sum a_{i}^{\prime}=m_{1}+1-p$ and $\sum a_{i}^{\prime \prime}=m_{2}+1-p$, and a set of homologous curves $\gamma_{i}, i=1, \ldots, p$ joining $z_{1}$ to $z_{2}$. The angle between $\gamma_{i}$ and $\gamma_{i+1}$ at $z_{1}$ is $2 \pi\left(a_{i}^{\prime}+1\right)$ and the angle between them at $z_{2}$ is $2 \pi\left(a_{i}^{\prime \prime}+1\right)$. We denote this 
assignment by

$$
\left(S^{\prime}, \gamma, a_{i}^{\prime}, a_{i}^{\prime \prime}\right) \rightarrow\left(S, m_{1}, m_{2}, a_{i}^{\prime}, a_{i}^{\prime \prime}\right)
$$

The resulting surface $\left(S, m_{1}, m_{2}, a_{i}^{\prime}, a_{i}^{\prime \prime}\right)$ has no saddle connections shorter than $\varepsilon$ other than the $\gamma_{i}^{\prime}$. Our convention on the choice of $\gamma_{i}^{\prime}, \gamma_{i}^{\prime \prime}$ (see the section above) implies that the cyclic order $\cdots \rightarrow \gamma_{i-1} \rightarrow \gamma_{i} \rightarrow \gamma_{i+1} \rightarrow \ldots$ is clockwise at $z_{1}$ with respect to the orientation defined by the flat structure.

Fix a basis for the relative homology on each surface $S_{i}^{\prime}$. A relative homology basis for $S$ is given by:

- A relative homology basis of curves $\beta_{i}$ for $S^{\prime}$.

- A curve connecting the two zeroes. The integral of $\omega$ along this curve is $\gamma$.
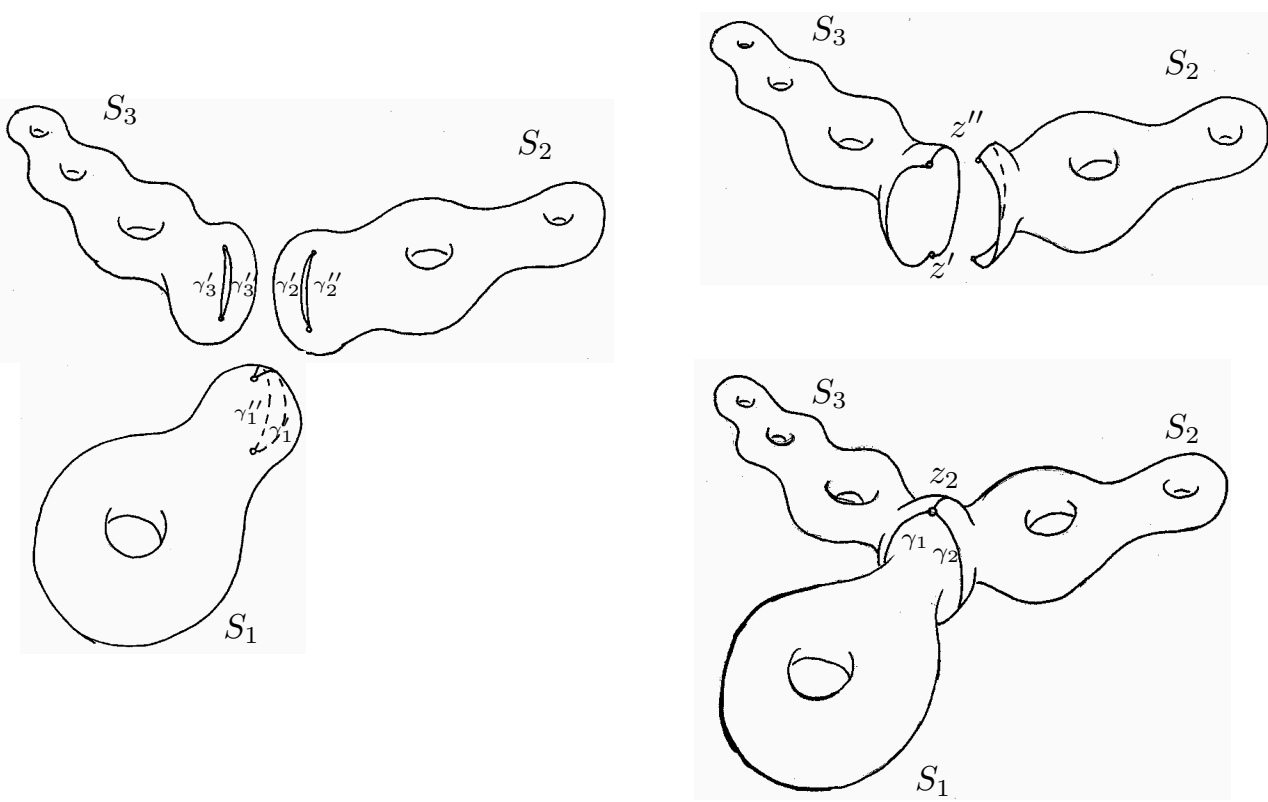

FigurE 5. Multiple homologous saddle connections.

We introduce $\bar{\alpha}_{i}^{\prime}$ as $\alpha^{\prime}$ from which we remove $a_{i}$.

Having a collection $\alpha$ of integers we denote by $|\alpha|$ their sum, say, $|\alpha|=2 g-2$.

The construction above implies the following conditions on partitions $\alpha_{i}^{\prime}$ and the numbers $a_{i}^{\prime}, a_{i}^{\prime \prime}$.

Lemma 9.1. An assignment $\left(S^{\prime}, \gamma, a_{i}^{\prime}, a_{i}^{\prime \prime}\right) \rightarrow\left(S, m_{1}, m_{2}, a_{i}^{\prime}, a_{i}^{\prime \prime}\right)$ satisfies the following necessary conditions on the collection $\left(\alpha_{i}^{\prime}, a_{i}^{\prime}, a_{i}^{\prime \prime}\right)$ :

$$
\begin{gathered}
a_{1}^{\prime}+\cdots+a_{p}^{\prime}=m_{1}+1-p \\
a_{1}^{\prime \prime}+\cdots+a_{p}^{\prime \prime}=m_{2}+1-p \\
\bar{\alpha}_{1}^{\prime} \sqcup \cdots \sqcup \bar{\alpha}_{p}^{\prime} \sqcup\left\{m_{1}\right\} \sqcup\left\{m_{2}\right\}=\alpha \\
\left|\bar{\alpha}_{i}^{\prime}\right|=a_{i}^{\prime}+a_{i}^{\prime \prime}(\bmod 2) \text { for } i=1, \ldots, p
\end{gathered}
$$

Moreover, when the stratum $\mathcal{H}_{1}(\alpha)$ is connected, these conditions are sufficient: every surface $S \in \mathcal{H}_{1}(\alpha)$ with a configuration of p homologous saddle connections joining the pair of zeroes of length at most $\varepsilon$ and no other saddle connection with length 


\begin{tabular}{c|ccc} 
Degeneration pattern & $\alpha_{1}^{\prime}$ & $\alpha_{2}^{\prime}$ & $\alpha_{3}^{\prime}$ \\
\hline & & & \\
$(0+0) \succ(1+1) \succ(0+1,2,1)$ & $(0)$ & $(2)$ & $(2,1,1)$ \\
$(1+1) \succ(0+0) \succ(0+1,2,1) \succ$ & $(2)$ & $(0)$ & $(2,1,1)$ \\
$(0+0) \succ(0+0) \succ(1+2,2,1) \succ$ & $(0)$ & $(0)$ & $(3,2,1)$ \\
$(0+2) \succ(0+0) \succ(1+0,2,1) \succ$ & $(2)$ & $(0)$ & $(2,1,1)$ \\
$(0+0) \succ(0+2) \succ(1+0,2,1) \succ$ & $(0)$ & $(2)$ & $(2,1,1)$ \\
$(1+1) \succ(0+1,1) \succ(0+0,2) \succ$ & $(2)$ & $(1,1)$ & $(2)$ \\
$(0+0) \succ(1+0,1) \succ(0+2,2)$ & $(0)$ & $(1,1)$ & $(2,2)$ \\
$(0+2) \succ(1+0,1) \succ(0+0,2)$ & $(2)$ & $(1,1)$ & $(2)$ \\
$(0+0) \succ(1+2,1) \succ(0+0,2)$ & $(0)$ & $(1,3)$ & $(2)$ \\
$(0+0) \succ(0+1,1) \succ(1+1,2)$ & $(0)$ & $(1,1)$ & $(2,2)$ \\
$(1+1) \succ(0+0,2) \succ(0+1,1)$ & $(2)$ & $(2)$ & $(1,1)$ \\
$(0+0) \succ(1+1,2) \succ(0+1,1)$ & $(0)$ & $(2,2)$ & $(1,1)$ \\
$(0+0) \succ(0+0,2) \succ(1+2,1)$ & $(0)$ & $(2)$ & $(1,3)$ \\
$(0+2) \succ(0+0,2) \succ(1+0,1)$ & $(2)$ & $(2)$ & $(1,1)$ \\
$(0+0) \succ(0+2,2) \succ(1+0,1) \succ$ & $(0)$ & $(2,2)$ & $(1,1)$
\end{tabular}

TABle 3. Possible degenerations for $S \in \mathcal{H}_{1}(4,3,2,1)$ with $m_{1}=$ $3, m_{2}=4 ; p=3$

smaller than $3 \varepsilon$ can be obtained by an assignment $\left(S^{\prime}, \gamma, a_{i}^{\prime}, a_{i}^{\prime \prime}\right) \rightarrow\left(S, m_{1}, m_{2}, a_{i}^{\prime}, a_{i}^{\prime \prime}\right)$ with appropriate $\left(S^{\prime}, \gamma, a_{i}^{\prime}, a_{i}^{\prime \prime}\right)$.

We shall introduce notation to describe the degeneration pattern for the surfaces $S_{i}$.

Notation. The integer represented as a sum of two integers corresponds to $a_{i}$ represented as $a_{i}^{\prime}+a_{i}^{\prime \prime}$. Here the order of appearances of the summands $a_{i}^{\prime}+a_{i}^{\prime \prime}$ is significant. The cyclic order of the surfaces is represented by $S_{1} \succ S_{2} \succ S_{3} \succ \ldots \succ$ $S_{p} \succ S_{1}$.

Example 9.2. Let $S \in \mathcal{H}_{1}(4,3,2,1) ; m_{1}=3, m_{2}=4 ; p=3$. There are 15 possible pictures for the three homologous saddle connections joining the zero of order 3 to the zero of order 4 depending on the returning angles (Table 3). Thus for example, the first line in table 3 refers to 3 surfaces in cyclic order. The first is a torus (no zeroes) where we have broken up a marked point into $(0+0)$; the second surface has a single zero of order 2 which we have broken into two simple zeroes indicated by $(1+1)$, and the third surface has zeroes of $2,1,1$, where we have broken one of the simple zeroes into a simple zero and a zero of order 0 .

9.4. Stratum Interchange and $\gamma \rightarrow-\gamma$ Symmetry. In this section we discuss the possible symmetries of the assignment

$$
\left(S^{\prime}, \gamma, a_{i}^{\prime}, a_{i}^{\prime \prime}\right) \rightarrow\left(S, m_{1}, m_{2}, a_{i}^{\prime}, a_{i}^{\prime \prime}\right)
$$

Let us first specify our problem.

Problem 1. We assume that the zeroes $z_{1}, \ldots, z_{l}$ of the surface $S$ are numbered. We fix the zeroes $z_{1}, z_{2} \in S, z_{1} \neq z_{2}$, of orders $m_{1}$ and $m_{2}$ correspondingly.

We start with the case when we fix also the following data. We assume that the zeroes $z_{3}, \ldots, z_{l_{1}}$ belong to $S_{1}$, the zeroes $z_{l_{1}+1}, \ldots, z_{l_{1}+l_{2}}$ belong to $S_{2}, \ldots$, the 
zeroes $z_{l_{1}+\cdots+l_{p-1}+1}, \ldots, z_{l_{1}+\cdots+l_{p}}$ belong to $S_{p}$. Here $l_{1}-1=\operatorname{card}\left(\alpha_{1}^{\prime}\right)$ if $a_{1} \neq 0$ and $l_{1}=\operatorname{card}\left(\alpha_{1}^{\prime}\right)$ when $a_{1}=0$ and for $i>1, l_{i}+1=\operatorname{card}\left(\alpha_{i}^{\prime}\right)$, when $a_{i} \neq 0$, and $l_{i}=\operatorname{card}\left(\alpha_{i}^{\prime}\right)$, when $a_{i}=0$. We have $l=l_{1}+\cdots+l_{p}=\operatorname{card}(\alpha)$.

In this setting the only thing that is not determined is the case when $S$ has exactly two zeroes: $z_{1}$ and $z_{2}$. Then every $S_{i}^{\prime}$ either has a single zero of order $a_{i}$ or it is a torus with a marked (regular) point. Note that we fix only the cyclic order of the collection $\left(a_{i}^{\prime}, a_{i}^{\prime \prime}\right)$, but not the numbering of each of these pair of numbers. The natural action of the cyclic group of order $p$ on the collection of ordered pairs $\left(a_{i}^{\prime}, a_{i}^{\prime \prime}\right)$ organized in a cyclic order, may have nontrivial stabilizer, which we denote by $\Gamma$. We get symmetry of order $|\Gamma|$. This type of symmetry we call the stratum interchange.

Problem 2. Now consider the problem with fewer constrains. We count the number of occurrences of the following phenomenon: some zero of $S$ of order $m_{1}$ is joined to some zero of order $m_{2}$ by precisely $p$ homologous saddle connections $\gamma_{1}, \ldots, \gamma_{p}$ as above. We fix the type $\alpha_{i}^{\prime}$ of the flat surface $S_{i}^{\prime}$ obtained as described above, but we make no assumptions on the distribution of the zeroes $z_{1}, \ldots, z_{l}$ by the surfaces $S_{i}^{\prime}$. As before we fix only the cyclic order of appearances of the surfaces $S_{i}$.

Remark 9.3. Problem 2 may be considered as generalization of Problem 1 to the case when the zeroes of $S$ and of $S_{i}^{\prime}$ are not numbered.

Remark 9.4. In both settings the $\mathcal{H}_{1}\left(\alpha_{i}^{\prime}\right)$ might be nonconnected. We could also specify the connected component of the $\mathcal{H}_{1}\left(\alpha_{i}^{\prime}\right)$ when this occurs. We prefer the setting where this data is not specified.

Consider the natural action of the cyclic group of order $p$ on the collection

$$
\left(\alpha_{1}^{\prime}, a_{1}^{\prime}, a_{1}^{\prime \prime}\right) \succ \cdots \succ\left(\alpha_{p}^{\prime}, a_{p}^{\prime}, a_{p}^{\prime \prime}\right) \succ
$$

organized in a cyclic order. (Recall the notation $\succ$ from the previous section). If it has a nontrivial stabilizer we denote it by the same symbol $\Gamma$. We get a symmetry of order $|\Gamma|$ which we also call the stratum interchange.

Example 9.5. Let $\alpha=(27,15,1,1), m_{1}=27, m_{2}=15$. Consider the degeneration of $S$ into 6 surfaces of the following types $\alpha_{1}^{\prime} \succ \cdots \succ \alpha_{6}^{\prime} \succ$

$$
(2) \succ(7,1) \succ(12) \succ(2) \succ(7,1) \succ(12) \succ
$$

The possible symmetry in this case is a symmetry of order 2 , where subgroup $\Gamma$ is a shift by 3 .

The degeneration

$$
(0+2) \succ(4+3,1) \succ(7+5) \succ(0+2) \succ(4+3,1) \succ(7+5) \succ
$$

possesses this symmetry, while the degeneration

$$
(0+2) \succ(3+4,1) \succ(7+5) \succ(2+0) \succ(4+3,1) \succ(7+5) \succ
$$

does not.

When $m_{1}=m_{2}$ we may have an additional symmetry in Problem 2. Let $P, Q$ be two zeroes of $\omega$ of order $m_{1}=m_{2}$ joined by precisely $p$ homologous saddle connections. Let the homology class of the saddle connection be represented by a vector $\gamma$. Assigning the "names" $z_{1}:=P$ and $z_{2}:=Q$ to $P$ and $Q$ we get a decomposition $\left(S_{i}^{\prime}, \gamma, \alpha_{i}^{\prime}, a_{i}^{\prime}, a_{i}^{\prime \prime}\right)$. 
Consider now the very same surface $S$ with the same configuration of $p$ homologous saddle connections joining the same two zeroes $P$ and $Q$. We may declare now that the correspondence of zeroes is inverse with respect to the previous assignment: $z_{1}:=Q$ and $z_{2}:=P$. Since our saddle connections are oriented (from $z_{1}$ to $z_{2}$ ) the homology class of the same saddle connection (from $P$ to $Q$ ) is represented now by the vector $-\gamma$. Since the cyclic order of "pieces" is determined by the cyclic order at the point $z_{1}$ the new identification reverses the cyclic order in the collection $\left(\alpha_{i}^{\prime}\right)$, as well as the order in the pairs $\left(a_{i}^{\prime}, a_{i}^{\prime \prime}\right)$.

We say, that we have a $\gamma \rightarrow-\gamma$ symmetry if and only if the assignment $z_{1}:=Q$ and $z_{2}:=P$ gives a decomposition with the same (up to a cyclic reenumeration) collection $\left(\alpha_{i}^{\prime}, a_{i}^{\prime}, a_{i}^{\prime \prime}\right)$ as before.

It is easy to see the following combinatorial criterion of the $\gamma \rightarrow-\gamma$ symmetry. Interchange simultaneously all $a_{i}^{\prime} \leftrightarrow a_{i}^{\prime \prime}$, and change the cyclic order of the resulting collection $\left(\alpha_{i}^{\prime}, a_{i}^{\prime \prime}, a_{i}^{\prime}\right)$ to the opposite one. We possess a $\gamma \rightarrow-\gamma$ symmetry if and only if the result of this operation gives us the initial collection $\left(\alpha_{i}^{\prime}, a_{i}^{\prime}, a_{i}^{\prime \prime}\right)$ up to a cyclic reenumeration. We denote $\gamma \rightarrow-\gamma$ symmetry by $\Gamma_{-}$, where $\left|\Gamma_{-}\right|=2$. We let $\left|\Gamma_{-}\right|=1$ when there is no $\gamma \rightarrow-\gamma$ symmetry.

The condition $m_{1}=m_{2}$ is an obvious necessary condition for $\gamma \rightarrow-\gamma$ symmetry. It will be always assumed in the discussions of $\gamma \rightarrow-\gamma$ symmetry.

Under this condition every degeneration of multiplicity 1 possesses a $\gamma \rightarrow-\gamma$ symmetry. There is a $\gamma \rightarrow-\gamma$ symmetry in multiplicity 2 if and only if at least one the following conditions is valid: either

- $a_{1}^{\prime}=a_{1}^{\prime \prime}$ (and then $a_{2}^{\prime}=a_{2}^{\prime \prime}$ automatically); or

- $\alpha_{1}^{\prime}=\alpha_{2}^{\prime}$ and $a_{1}^{\prime}=a_{2}^{\prime \prime}$ (and then $a_{1}^{\prime \prime}=a_{2}^{\prime}$ automatically).

Example 9.6. Let $\alpha=(11,11)$. Consider degeneration of $S$ into 4 surfaces of the following types $\alpha_{1}^{\prime} \succ \alpha_{2}^{\prime} \succ \alpha_{3}^{\prime} \succ \alpha_{4}^{\prime}$ :

$$
(2) \succ(6) \succ(2) \succ(6) \succ
$$

The degeneration

$$
(0+2) \succ(3+3) \succ(2+0) \succ(3+3) \succ
$$

possesses the $\gamma \rightarrow-\gamma$ symmetry, but does not have the stratum interchange, so $\left|\Gamma_{-}\right|=2,|\Gamma|=1$.

The degeneration

$$
(0+2) \succ(4+2) \succ(0+2) \succ(4+2) \succ
$$

does not have a $\gamma \rightarrow-\gamma$ symmetry, but allows the stratum interchange, so $\left|\Gamma_{-}\right|=1$, $|\Gamma|=2$.

The degeneration

$$
(1+1) \succ(3+3) \succ(1+1) \succ(3+3) \succ
$$

has both symmetries: $\left|\Gamma_{-}\right|=2,|\Gamma|=2$.

Remark 9.7. Describing the assignments $\left(S^{\prime}, \gamma, a_{i}^{\prime}, a_{i}^{\prime \prime}\right) \rightarrow\left(S, m_{1}, m_{2}, a_{i}^{\prime}, a_{i}^{\prime \prime}\right)$ one can consider all possible assignments, and then take into account corresponding symmetries, or one can deal with the classes, eliminating the symmetry whenever it is possible.

We have chosen the second way. For example the assignment

$$
(2+0) \succ(0+0) \succ(0+1,2,1)
$$


is not presented in the Table 3 since it is symmetric to the assignment

$$
(0+0) \succ(0+2) \succ(1+0,2,1)
$$

(which is in the list) by composition of $\gamma \rightarrow-\gamma$ symmetry with the stratum interchange.

Let us return to the degeneration construction described in Section 9.1 To every surface $S$ in the thick part $\mathcal{H}_{1}^{\varepsilon, \text { thick }}(\alpha, \mathcal{C})$ we associate a surface $S^{\prime}=\sqcup_{i=1}^{p} S_{i}^{\prime}$. We denote the corresponding stratum by $\mathcal{H}\left(\alpha^{\prime}\right)$, where $\alpha^{\prime}=\sqcup \alpha_{i}^{\prime}$, see Convention [5

However, our consideration of the symmetries shows, that the surface $S^{\prime}$ is defined up to a finite symmetry. In other words, we may have a nontrivial symmetry group $\Gamma_{ \pm}$acting on the stratum $\mathcal{H}\left(\alpha^{\prime}\right)$. This symmetry group is generated by the subgroup $\Gamma$ of stratum interchange symmetries and subgroup $\Gamma_{-}$of $\gamma \rightarrow-\gamma$ symmetries. (Any of the subgroups, or both might be trivial.) The order $\left|\Gamma_{ \pm}\right|$of the symmetry group $\Gamma_{ \pm}$is equal to the product of the orders $|\Gamma|$ and $\left|\Gamma_{-}\right|$.

Note that the symmetry group $\Gamma_{ \pm}$preserves the natural measure on $\mathcal{H}\left(\alpha^{\prime}\right)$. It obviously preserves the area of the surface, hence $\Gamma_{ \pm}$preserves the "unit hyperboloid" $\mathcal{H}_{1}\left(\alpha^{\prime}\right)$. Thus we get a natural volume element on the quotient $\mathcal{H}_{1}\left(\alpha^{\prime}\right) / \Gamma_{ \pm}$.

The degeneration construction can be considered as a map

$$
\mathcal{H}_{1}^{\varepsilon, \text { thick }}(\alpha, \mathcal{C}) \rightarrow\left(\mathcal{H}_{1}\left(\alpha^{\prime}\right) / \Gamma_{ \pm}\right) \times B(\varepsilon)
$$

In the next section we shall study this map. This will allow us to compute the volume of the thick part and thus compute the corresponding Siegel-Veech constant.

9.5. Siegel-Veech Constants in Higher Multiplicity, Connected Strata. Consider the set $\mathcal{H}_{1}^{\varepsilon, t h i c k}(\alpha, \mathcal{C})$. It consists of surfaces $S$ having exactly one collection of short homologous saddle connections. This collection is necessarily of the type $\mathcal{C}$; the saddle connections from this collection (which are all of the same length) are shorter then $\varepsilon$; any other saddle connection on $S$ is longer then $\kappa$.

Actually, we shall need two variants of this set: the one, which we denote $\mathcal{H}_{1}^{\varepsilon, \varepsilon}(\alpha, \mathcal{C})$ corresponds to the value $\kappa=\varepsilon$ of parameter $\kappa$; the other, denoted $\mathcal{H}_{1}^{\varepsilon, 3 \varepsilon}(\alpha, \mathcal{C})$, corresponds to $\kappa=3 \varepsilon$.

Clearly,

$$
\mathcal{H}_{1}^{\varepsilon, 3 \varepsilon}(\alpha, \mathcal{C}) \subset \mathcal{H}_{1}^{\varepsilon, \varepsilon}(\alpha, \mathcal{C})
$$

Lemma 9.8. Consider the map

$$
p: \mathcal{H}_{1}^{\varepsilon, \varepsilon}(\alpha, \mathcal{C}) \rightarrow \mathcal{H}_{1}\left(\alpha^{\prime}\right) / \Gamma_{ \pm} \times B(\varepsilon)
$$

corresponding to the assignment $\left(S^{\prime}, \gamma, a_{i}^{\prime}, a_{i}^{\prime \prime}\right) \rightarrow\left(S, m_{1}, m_{2}, a_{i}^{\prime}, a_{i}^{\prime \prime}\right)$. Let

$$
\mathcal{F}_{2 \varepsilon}^{\prime}:=\left(\mathcal{H}_{1}\left(\alpha^{\prime}\right)-\mathcal{H}_{1}^{2 \varepsilon}\left(\alpha^{\prime}\right)\right) / \Gamma_{ \pm} .
$$

1. The image of the map $p$ contains the subset $\mathcal{F}_{2 \varepsilon}^{\prime} \times B(\varepsilon)$.

2. Let $\mathcal{U}:=p^{-1}\left(\mathcal{F}_{2 \varepsilon}^{\prime} \times B(\varepsilon)\right)$. The restriction $\left.p\right|_{\mathcal{U}}$ is a (ramified) covering of degree

$$
M_{0}:=\prod_{i=1}^{p}\left(a_{i}+1\right)
$$


where $a_{i}=a_{i}^{\prime}+a_{i}^{\prime \prime}$ is the degree of the distinguished zero on the surface $S_{i}^{\prime}$.

3. The map $\left.p\right|_{\mathcal{U}}$ is volume preserving: the volume element on $\mathcal{U}$ induced from $d \operatorname{vol}^{\prime} \times d \gamma$ on $\mathcal{F}_{2 \varepsilon}^{\prime} \times B(\varepsilon)$ coincides with the volume element $d \operatorname{vol}$ on $\mathcal{U} \subset \mathcal{H}_{1}(\alpha)$.

4. The set $\mathcal{U}$ contains the set $\mathcal{H}_{1}^{\varepsilon, 3 \varepsilon}(\alpha, \mathcal{C})$.

Proof. In fact, the statement of the Lemma is almost tautological reformulation in geometric terms of the properties which we already know.

Part 1 follows immediately from the construction in the beginning of Section 9.3 , where having a surface $S^{\prime} \in \mathcal{H}_{1}\left(\alpha^{\prime}\right)-\mathcal{H}_{1}^{2 \varepsilon}\left(\alpha^{\prime}\right)$ and a vector $\gamma \in B(\varepsilon)$ we constructed a surface $S \in \mathcal{H}_{1}^{\varepsilon, \varepsilon}(\alpha, \mathcal{C})$. We have $p(S)=S^{\prime}$.

The fact that $p$ is nondegenerate almost everywhere follows immediately from consideration of $p$ in cohomological coordinates, so in this sense it is a (ramified) covering. To prove statement 2 it remains to compute the degree of this covering, i.e. to prove that for almost all pairs $\left(S^{\prime}, \gamma\right)$ we can construct exactly $M_{0}$ pairwise nonisometric surfaces. For each surface $S_{i}^{\prime}$ there are $a_{i}+1$ ways to perform a slit construction at a zero of order $a_{i}=a_{i}^{\prime}+a_{i}^{\prime \prime}$ of $S_{i}^{\prime}$ providing $a_{i}+1$ surfaces $S_{i}$. According to Lemma 8.1 for almost all $S^{\prime} \in \mathcal{F}_{2 \varepsilon}^{\prime}$ the resulting $a_{i}+1$ surfaces $S_{i}$ are pairwise nonisometric. For almost all $S^{\prime}$ the surfaces $S_{i}$ and $S_{j}$ corresponding to different indices $i \neq j$ are nonisometric as well. Thus generically there are no isomorphisms between the resulting surfaces $S$ which map the collection $\left\{\gamma_{i}\right\}$ on one surface to the collection $\left\{\gamma_{i}\right\}$ on the other surface. Since by the choice of $\left(S^{\prime}, \gamma\right)$ such a collection is unique on each of the $M_{0}$ surfaces $S$, they are generically pairwise nonisometric.

Statement 3 follows immediately from the fact that as a basis of cycles in $H_{1}\left(S,\left\{P_{1}, \ldots, P_{k}\right\} ; \mathbb{Z}\right)$ we can choose a union of basic cycles for $S_{i}^{\prime}$ and one of the homologous saddle connections $\gamma_{i}$, where by definition $\operatorname{hol}\left(\gamma_{i}\right)=\gamma$. Thus, this statement is completely analogous to the corresponding statement in multiplicity one.

Statement 4 follows from the fact that the surgery, which associates a surface $S^{\prime}$ to a surface $S$ either does not change the holonomy of saddle connections different from $\gamma_{i}$ or changes it by holonomy of $\gamma_{i}$. Since $\left|\gamma_{i}\right|<\varepsilon$ it means that a saddle connection, which is longer than $3 \varepsilon$ would stay longer than $2 \varepsilon$ after the surgery.

Corollary 9.9. Suppose that the stratum $\mathcal{H}(\alpha)$ is connected; let $\mathcal{C}$ be an admissible configuration of saddle connections joining a pair of distinct zeroes; let $\left(S^{\prime}, \gamma, a_{i}^{\prime}, a_{i}^{\prime \prime}\right) \rightarrow\left(S, m_{1}, m_{2}, a_{i}^{\prime}, a_{i}^{\prime \prime}\right)$ be the corresponding assignment. Then

$$
\operatorname{Vol}\left(\mathcal{H}_{1}^{\varepsilon}(\alpha, \mathcal{C})\right)=M \cdot \operatorname{Vol}\left(\mathcal{H}_{1}\left(\alpha^{\prime}\right)\right) \cdot \pi \varepsilon^{2}+o\left(\varepsilon^{2}\right),
$$

where the integer constant $M$ is defined as

$$
M=\frac{\prod_{i=1}^{p}\left(a_{i}+1\right)}{\left|\Gamma_{-}\right| \cdot|\Gamma|}
$$

Here $a_{i}=a_{i}^{\prime}+a_{i}^{\prime \prime} ;\left|\Gamma_{-}\right|=2$ if the assignment has $\gamma \rightarrow-\gamma$ symmetry and $\left|\Gamma_{-}\right|=1$ otherwise; $|\Gamma|$ is the order of the "stratum interchange" symmetry.

Proof. Combining statement 4 of the Lemma above with the obvious inclusions (19) we obtain the following inclusions

$$
\mathcal{H}_{1}^{\varepsilon, \text { thick }}(\alpha, \mathcal{C})=\mathcal{H}_{1}^{\varepsilon, 3 \varepsilon}(\alpha, \mathcal{C}) \subset \mathcal{U} \subset \mathcal{H}_{1}^{\varepsilon}(\alpha, \mathcal{C})
$$

Hence, by Corollary 7.2

$$
\operatorname{Vol}\left(\mathcal{H}_{1}^{\varepsilon}(\alpha, \mathcal{C})\right)=\operatorname{Vol}(\mathcal{U})+o\left(\varepsilon^{2}\right) .
$$


On the other hand, by statements 2 and 3 of the Lemma above,

$$
\operatorname{Vol}(\mathcal{U})=M_{0} \cdot \operatorname{Vol}\left(\mathcal{F}_{2 \varepsilon}^{\prime}\right) \cdot \operatorname{Vol}(B(\varepsilon))
$$

The volume of the disc $B(\varepsilon)$ equals $\pi \varepsilon^{2}$. The volume of the quotient over a finite group of isometries equals to the volume of the total space divided by the order of the group. Since $\left|\Gamma_{ \pm}\right|=\left|\Gamma_{-}\right| \cdot|\Gamma|$, and the set $\mathcal{F}_{2 \varepsilon}^{\prime}$ is defined as

$$
\mathcal{F}_{2 \varepsilon}^{\prime}:=\left(\mathcal{H}_{1}\left(\alpha^{\prime}\right)-\mathcal{H}_{1}^{2 \varepsilon}\left(\alpha^{\prime}\right)\right) / \Gamma_{ \pm}
$$

we obtain

$$
\operatorname{Vol}\left(\mathcal{F}_{2 \varepsilon}^{\prime}\right)=\left(\operatorname{Vol}\left(\mathcal{H}_{1}\left(\alpha^{\prime}\right)\right)-\operatorname{Vol}\left(\mathcal{H}_{1}^{2 \varepsilon}\left(\alpha^{\prime}, \mathcal{C}\right)\right)\right) /\left(\left|\Gamma_{-}\right| \cdot|\Gamma|\right)
$$

It follows from Lemma 7.1 that $\operatorname{Vol}\left(\mathcal{H}_{1}^{2 \varepsilon}\left(\alpha^{\prime}\right)\right)=O\left(\varepsilon^{2}\right)$. Hence,

$$
\operatorname{Vol}\left(\mathcal{F}_{2 \varepsilon}^{\prime}\right)=\operatorname{Vol}\left(\mathcal{H}_{1}\left(\alpha^{\prime}\right)\right) /\left(\left|\Gamma_{-}\right| \cdot|\Gamma|\right)+O\left(\varepsilon^{2}\right)
$$

Summarizing the above arguments we obtain the statement of the corollary.

Now everything is ready to compute the Siegel - Veech constant $c(\mathcal{C})$. Note that we work in the setting where we fix the partition of numbered zeroes of $S$ by components $S_{i}^{\prime}$ (see Problem 1 in section 9.4). We assume that $\mathcal{H}_{1}(\alpha)$ is connected. Applying Proposition 3.3 we get the following value of the $c(\mathcal{C})$ :

$$
c(\mathcal{C})=M \cdot \frac{\operatorname{Vol}\left(\mathcal{H}_{1}\left(\alpha^{\prime}\right)\right)}{\operatorname{Vol}\left(\mathcal{H}_{1}(\alpha)\right)}
$$

Using expression (21) for $M$ and Formula (12) from Section 6.2 for the volume of a nonprimitive stratum $\mathcal{H}_{1}\left(\alpha^{\prime}\right)$ we finally obtain the following formula:

$$
c=\frac{1}{|\Gamma|} \frac{1}{\left|\Gamma_{-}\right|} \cdot \prod_{j=1}^{p}\left(a_{j}+1\right) \cdot \frac{1}{2^{p-1}} \cdot \frac{\prod_{i=1}^{p}\left(\frac{d_{i}}{2}-1\right) !}{\left(\frac{d}{2}-2\right) !} \cdot \frac{\prod_{i=1}^{p} \operatorname{Vol}\left(\mathcal{H}_{1}\left(\alpha_{i}\right)\right)}{\operatorname{Vol}\left(\mathcal{H}_{1}(\alpha)\right)}
$$

where

$$
d_{i}=\operatorname{dim}_{\mathbb{R}} \mathcal{H}_{1}\left(\alpha_{i}^{\prime}\right)
$$

Note, that if $a_{i}=0$, the corresponding surface $S_{i}^{\prime}$ has a marked point, so $\alpha_{i}^{\prime}$ contains 0 . Note also, that by convention when $S_{i}^{\prime}$ is a torus, we use the point which is already marked, so $\alpha_{i}^{\prime}=0$.

Let us study now the constant $c$ in the setting where we do not specify which zeroes $S$ are to be joined. (see Problem 2 in Section 9.4). This counting problem can be reduced to the previous one by a purely combinatorial computation. Consider $m \neq m_{1}, m \neq m_{2}, m \neq a_{i}, i=1, \ldots, p$. Then these zeroes are all "inherited" by the surfaces $S_{i}$. Since all the zeroes $z_{1}, z_{2}, \ldots, z_{l}$ are named, the number of ways to distribute $o(m)$ zeroes of order $m$ into groups of $o_{1}(m), \ldots, o_{p}(m)$ zeroes equals

$$
\frac{o(m) !}{\prod_{i=1}^{p} o_{i}(m) !}
$$

If $m \neq m_{1}$ and $m \neq m_{2}$ but $m=a_{j}$ for some $j$ then one of the $o_{j}(m)$ zeroes of order $m$ which lives on $S_{j}$ is newborn; that is, arising from the degeneration, while the other $o_{j}(m)-1$ zeroes of order $m$ come from the corresponding zeroes of order 
$m$ on $S$. Thus the corresponding factor in the denominator becomes $\left(o_{j}(m)-1\right)$ !. Multiplying numerator and denominator by $o_{j}(m)$ we get the following factor:

$$
\frac{o(m) !}{\prod_{j=1}^{p} o_{j}(m) !} \cdot \prod_{j \mid a_{j}=m} o_{j}(m)
$$

(Equivalently, we may say that we have performed the slit construction at the first "named" zero of order $a_{j}$ on $S_{j}$. The rest of the zeroes of that order inherit their names on $S$, but then we count the number of ways of merging the names from different $S_{i}$ onto $S$.) Consider now the case when $m=m_{1}$ or $m=m_{2}$. When $m_{1} \neq m_{2}$ and $m=m_{1}$ (resp. $m=m_{2}$ ) we have the arrangements of the other $o\left(m_{1}\right)-1$ (resp. $\left.o\left(m_{2}\right)-1\right)$ zeroes of order $m$. When $m_{1}=m_{2}$ we have an arrangement of the other $o\left(m_{1}\right)-2$ zeroes of order $m$. This changes the numerator in the formula above by the corresponding factorial. However, in these cases we have to count the number of ways to choose a zero of degree $m_{1}$ and a zero of degree $m_{2}$ on the initial surface $S$. This number is equal to $o\left(m_{1}\right) \cdot o\left(m_{2}\right)$ if $m_{1} \neq m_{2}$ and $o\left(m_{1}\right)\left(o\left(m_{1}\right)-1\right)$ if $m_{1}=m_{2}$. Thus for zeroes of these degrees we have this extra factor $o\left(m_{1}\right) \cdot o\left(m_{2}\right)$ which combined with previous considerations gives $o(m)$ ! in this case as well. Taking into account the possible symmetries (see section 9.4), we finally get the following expression for the constant $c$ in the setting where the zeroes of $S$ are not numbered (see Problem 2 in section 9.4).

\section{Formula 9.1.}

$$
\begin{aligned}
c=\frac{1}{\left|\Gamma_{-}\right|} \cdot \frac{1}{|\Gamma|} \cdot \prod_{m \in \alpha} & \left(\frac{o(m) !}{\prod_{j=1}^{p} o_{j}(m) !}\right) \cdot \prod_{\substack{j=1 \\
a_{j} \neq 0}}^{p} o_{j}\left(a_{j}\right) . \\
& \cdot \prod_{j=1}^{p}\left(a_{j}+1\right) \cdot \frac{1}{2^{p-1}} \cdot \frac{\prod_{i=1}^{p}\left(\frac{d_{i}}{2}-1\right) !}{\left(\frac{d}{2}-2\right) !} \cdot \frac{\prod_{i=1}^{p} \operatorname{Vol}\left(\mathcal{H}_{1}\left(\alpha_{i}\right)\right)}{\operatorname{Vol}\left(\mathcal{H}_{1}(\alpha)\right)}
\end{aligned}
$$

We give some examples of this formula in the case of connected strata $\mathcal{H}_{1}(\alpha)$.

Example 9.10. Stratum $\mathcal{H}_{\mathbf{1}}(\mathbf{3}, \mathbf{1})$; The multiplicity is $p=2$. After degeneration we get a surface $S_{1}^{\prime}$ of genus 2 with a single zero of order $a_{1}=2$, where now $a_{1}^{\prime}=2, a_{1}^{\prime \prime}=0$, and we get a torus $S_{2}^{\prime}$, with $a_{2}=a_{2}^{\prime}=a_{2}^{\prime \prime}=0$.

The saddle connections $\gamma_{1}$ and $\gamma_{2}$ partition the cone angle $8 \pi$ at $z_{1}$ in two sectors with angles $2 \pi$ and $6 \pi$; they partition the cone angle $4 \pi$ at $z_{2}$ in two sectors with angles $2 \pi$ and $2 \pi$. There is no stratum interchange, so $|\Gamma|=1$. We get

$$
c=3 \cdot \frac{1}{2} \cdot \frac{3 ! \cdot 1 !}{5 !} \frac{\operatorname{Vol}\left(\mathcal{H}_{1}(2)\right) \cdot \operatorname{Vol}\left(\mathcal{H}_{1}(0)\right)}{\operatorname{Vol}\left(\mathcal{H}_{1}(3,1)\right)}=\frac{567}{1024} \approx 0.554
$$

Example 9.11. Stratum $\mathcal{H}_{\mathbf{1}}(\mathbf{2}, \mathbf{1}, \mathbf{1})$; genus $g=3$, multiplicity $p=2$. First suppose that we have a pair of homologous saddle connections joining the zero of degree $m_{1}=2$ with one of two zeroes of degree $m_{2}=1$. After degeneration we get two surfaces $S_{1}^{\prime} \in \mathcal{H}_{1}\left(a_{1}, 1\right)$, and $S_{2}^{\prime} \in \mathcal{H}_{1}\left(a_{2}\right)$, where $a_{1}+a_{2}=m_{1}+m_{2}+2-2 p=1$. Thus $a_{1}=1, a_{2}=0$. There is no stratum interchange, so $|\Gamma|=1$. Altogether we get

$$
c=4 \cdot \frac{1}{2} \cdot \frac{4 ! \cdot 1 !}{6 !} \cdot \frac{\operatorname{Vol}\left(\mathcal{H}_{1}(1,1)\right) \operatorname{Vol}\left(\mathcal{H}_{1}(0)\right)}{\operatorname{Vol}\left(\mathcal{H}_{1}(2,1,1)\right)}=\frac{28}{45} \approx 0.622
$$

Suppose now that we have a pair of homologous saddle connections joining two simple zeroes. After degeneration we get two surfaces $S_{1}^{\prime} \in \mathcal{H}\left(a_{1}, 2\right)$, and $S_{2}^{\prime} \in$ 
$\mathcal{H}\left(a_{2}\right)$, where $a_{1}+a_{2}=m_{1}+m_{1}+2-2 p=0$. Thus $S^{\prime}$ is genus 2 with a marked point and with a zero of degree 2 , while $S_{2}^{\prime}$ is a torus with a marked point. Here we have $a_{i}^{\prime}=a_{i}^{\prime \prime}=0, i=1,2$.

There is no stratum interchange, so $|\Gamma|=1$. We now get

$$
c=\frac{1}{2} \cdot 1 \cdot \frac{4 ! \cdot 1 !}{6 !} \cdot \frac{\operatorname{Vol}\left(\mathcal{H}_{1}(2)\right) \operatorname{Vol}\left(\mathcal{H}_{1}(0)\right)}{\operatorname{Vol}\left(\mathcal{H}_{1}(2,1,1)\right)}=\frac{7}{40}=0.175
$$

9.6. Principal Stratum. The cone angle at any simple zero equals $4 \pi$. Thus for the principal stratum we may have at most two homologous saddle connections joining a pair of distinct zeroes. Hence in the higher multiplicity case, the surface $S, \in \mathcal{H}_{1}(1, \ldots, 1)$ of genus $g$ degenerates to a pair of surfaces $S_{1}^{\prime}, S_{2}^{\prime}$ of genera $g_{1}+$ $g_{2}=g$. Each of $S_{1}^{\prime}, S_{2}^{\prime}$ has only simple zeroes and one additional marked point. We always have the $\gamma \rightarrow-\gamma$ symmetry, so $\left|\Gamma_{-}\right|=2$. When $g_{1}=g_{2}$ we have a symmetry $|\Gamma|=2$ due to the stratum interchange; there is no other symmetry otherwise.

Applying formula (23) and (22) we finally get the following answer.

Formula 9.2. The constant for the number of saddle connections of multiplicity two joining two distinct zeroes in the principal stratum is

$$
\begin{aligned}
& c=\frac{1}{2|\Gamma|} \cdot \frac{(2 g-2) !}{\left(2 g_{1}-2\right) !\left(2 g_{2}-2\right) !} \cdot 1 \cdot 1 \cdot \frac{1}{2^{2-1}} \cdot \frac{\left(4 g_{1}-3\right) !\left(4 g_{2}-3\right) !}{(4 g-5) !} . \\
& \cdot \frac{\operatorname{Vol}\left(\mathcal{H}_{1}(\overbrace{1, \ldots, 1}^{2 g_{1}-2})\right) \operatorname{Vol}\left(\mathcal{H}_{1}(\overbrace{1, \ldots, 1}^{2 g_{2}-2})\right)}{\operatorname{Vol}\left(\mathcal{H}_{1}(\underbrace{1, \ldots, 1}_{2 g-2})\right)}= \\
& =\frac{1}{4|\Gamma|} \cdot \frac{(2 g-2) !\left(4 g_{1}-3\right) !\left(4 g_{2}-3\right) !}{\left(2 g_{1}-2\right) !\left(2 g_{2}-2\right) !(4 g-5) !} \cdot \frac{\operatorname{Vol}\left(\mathcal{H}_{1}(\overbrace{1, \ldots, 1}^{2 g_{1}-2})\right) \operatorname{Vol}\left(\mathcal{H}_{1}(\overbrace{1, \ldots, 1}^{2 g_{2}-2})\right)}{\operatorname{Vol}\left(\mathcal{H}_{1}(\underbrace{1, \ldots, 1}_{2 g-2})\right)} \\
& \text { where } g_{1}+g_{2}=g, g_{1}, g_{2} \geq 1, \quad|\Gamma|= \begin{cases}1 & \text { when } g_{1} \neq g_{2} \\
2 & \text { when } g_{1}=g_{2}\end{cases}
\end{aligned}
$$

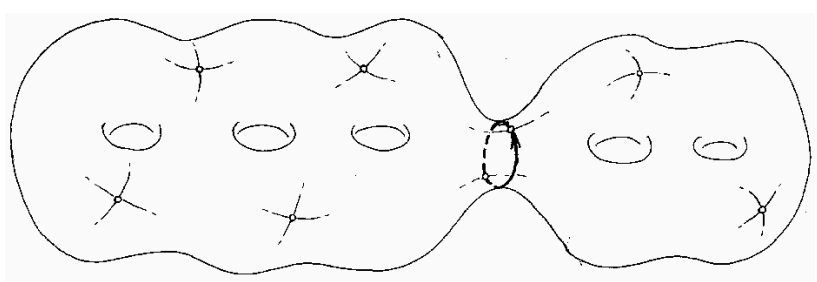

FiguRE 6. Saddle connection of multiplicity 2 on a surfaces from the principal stratum $\mathcal{H}_{1}(1, \ldots, 1)$.

Table 4 presents the numerical values for these constants. More values are presented in Table 8 in the Appendix. (We present only approximate values of constants in Table 8 just to save the space.) 
TABLE 4. Principal stratum; values of the constants for saddle connections of multiplicity two joining a pair of distinct zeroes

\begin{tabular}{|c|c|c|c|c|c|}
\hline & $g_{2}=1$ & $g_{2}=2$ & $g_{2}=3$ & $g_{2}=4$ & $g_{2}=5$ \\
\hline$g_{1}=1$ & $\frac{5}{8}$ & $\frac{6}{7}$ & $\frac{315}{377}$ & $\frac{19604}{23357}$ & $\frac{13897415}{16493303}$ \\
\hline$g_{1}=2$ & $\frac{6}{7}$ & $\frac{30}{377}$ & $\frac{1680}{23357}$ & $\frac{686140}{16493303}$ & $\frac{9529656}{351964697}$ \\
\hline$g_{1}=3$ & $\frac{315}{377}$ & $\frac{1680}{23357}$ & $\frac{154350}{16493303}$ & $\frac{352872}{50280671}$ & $\frac{389127620}{121780991823}$ \\
\hline$g_{1}=4$ & $\frac{19604}{23357}$ & $\frac{686140}{16493303}$ & $\frac{352872}{50280671}$ & $\frac{336277214}{365342975469}$ & $\frac{435911877856}{704782787198207}$ \\
\hline$g_{1}=5$ & $\frac{13897415}{16493303}$ & $\frac{9529656}{351964697}$ & $\frac{389127620}{121780991823}$ & $\frac{435911877856}{704782787198207}$ & $\frac{19865637635886}{249020069093788675}$ \\
\hline
\end{tabular}

\section{Strata Having Several Connected Components}

The analysis of admissible assignments, structure of local surgeries, and most of the elements of our constructions are basically the same for the strata having several connected components. However, we need to establish the correspondence between connected components and parts of the boundary of the stratum they are adjacent to. In other words we need to describe the admissible constructions, which lead to the flat surfaces in the prescribe connected component.

10.1. Parity of the Spin Structure in the Slit Construction. We start with outlining the relation between the parities of spin structures of the resulting surface and its components in the slit construction.

Lemma 10.1. Suppose $S$ constructed above has only even zeroes so has a spin structure. Then each component surface $S_{i}^{\prime}$ has only even zeroes and so also has a spin structure. The parity of the spin structure $\phi(S)$ is equal to the sum of the parities of the spin structures of the components:

$$
\phi(S)=\sum_{i=1}^{p} \phi\left(S_{i}^{\prime}\right)(\bmod 2)
$$

Proof. For each $S_{i}^{\prime}$, all the zeroes except the distinguished one come from the corresponding zeroes on $S$ unchanged; in particular they have the same degrees as the initial zeroes of $S$. Thus for each $S_{i}^{\prime}$ all the zeroes but at most one, have even degrees. Note that the total sum of the degrees of the zeroes of $S_{i}^{\prime}$ is even: it equals $2 g_{i}+2$, where $g_{i}$ is the genus of the surface $S_{i}^{\prime}$. Hence the remaining zero has even degree as well. (By usual convention we consider a marked point as a "zero of degree 0".)

For each surface $S_{i}^{\prime}$ consider a collection of $2 g_{i}$ smooth simple closed curves on $S_{i}^{\prime}$ representing the canonical basis of cycles. Breaking up the zero $z_{i}$ we make a local surgery of the flat structure, i.e., we do not change the flat structure outside of a small domain $U_{i}^{\prime} \subset S_{i}^{\prime}$ containing the zero $z_{i}$. Thus for each $i$ we can deform if necessary the curves from the corresponding collection in such a way that they stay outside of a neighborhood of the zero $z_{i}$. Thus we may assume that for each $i=1, \ldots, p$ none of the chosen curves on $S_{i}^{\prime}$ intersect $U_{i}^{\prime}$. This means that all the 
curves survive under the surgery, moreover, the union of collections for all $i$ gives us a canonical basis on $S$. By construction the index of any resulting curve in the flat structure $\omega$ is the same as the index of the corresponding curve in the flat structure $\omega_{i}^{\prime}$. Hence we get the desired relation (24). The lemma is proved.

Lemma 10.2. Let $S$ belong to one of the components $\mathcal{H}_{1}^{\text {odd }}(\alpha)$ or $\mathcal{H}_{1}^{\text {even }}(\alpha)$. Suppose that $\alpha \neq(g-1, g-1)$. Then the surface $S$ can be obtained by an assignment $\left(S^{\prime}, \gamma, a_{i}^{\prime}, a_{i}^{\prime \prime}\right) \rightarrow\left(S, m_{1}, m_{2}, a_{i}^{\prime}, a_{i}^{\prime \prime}\right)$, where $m_{1}, m_{2} \in \alpha$, if and only if the collection $\left(\alpha_{i}^{\prime}, a_{i}^{\prime}, a_{i}^{\prime \prime}\right)$ satisfies the conditions of Lemma 0.1 all $\alpha_{i}^{\prime}$ are even, and the parities of the spin structures of $S_{i}^{\prime}$ satisfy the condition of Lemma 10.1.

10.2. Admissible Assignments for Surfaces from Hyperelliptic Components. Note that the surfaces $S_{i}^{\prime}$ from the hyperelliptic component $\mathcal{H}_{1}^{\text {hyp }}(2 g-2)$ and from the hyperelliptic component $\mathcal{H}_{1}^{\text {hyp }}(g-1, g-1)$, where $g$ is odd, also have parity of the spin structure (see formulae (8), (9)). In general these surfaces are also involved in assignments from Lemma 10.2 producing nonhyperelliptic components $\mathcal{H}_{1}^{\text {even }}(\alpha)$ and $\mathcal{H}_{1}^{\text {odd }}(\alpha)$. However, in the exceptional case of $\alpha=(g-1, g-1)$ with odd $g$ some assignments lead to surfaces $S$ from the hyperelliptic connected component $\mathcal{H}_{1}^{\text {hyp }}(g-1, g-1)$. These assignments are classified in the lemma below.

Lemma 10.3. Let a flat surface $S$ from the hyperelliptic connected component $\mathcal{H}_{1}^{\text {hyp }}(g-1, g-1)$ be obtained by an assignment

$$
\left(S^{\prime}, \gamma, a_{i}^{\prime}, a_{i}^{\prime \prime}\right) \rightarrow\left(S, g-1, g-1, a_{i}^{\prime}, a_{i}^{\prime \prime}\right) .
$$

Then for almost every $S$ the assignment has one of the following two types:

- The multiplicity is 1 ; the flat surface $S^{\prime}$ belongs to the hyperelliptic component $\mathcal{H}_{1}^{\text {hyp }}(2 g-2)$, and $a^{\prime}=a^{\prime \prime}=g-1$.

- The multiplicity is 2 ; the flat surfaces $S_{i}^{\prime}, i=1,2$ belong to the hyperelliptic components $\mathcal{H}^{\text {hyp }}\left(2 g_{i}-2\right)$, where $g_{1}+g_{2}=g$, and $a_{i}^{\prime}=a_{i}^{\prime \prime}=g_{i}-1, i=1,2$.

The assignment is $|\Gamma|$ to 1 and is onto the corresponding set of hyperelliptic surfaces.

Proof. The set of hyperelliptic structures such that the involution interchanges some components has positive codimension, so we may disregard these and assume the involution fixes each component. This implies that all flat surfaces $S_{i}^{\prime}$ belong to components $\mathcal{H}^{\text {hyp }}\left(2 g_{i}-2\right)$. Since the hyperelliptic involution sends a vector $v$ to $-v$, but does not change the orientation of the surface, if the multiplicity is greater than one, it cannot map any of the $\gamma_{i}^{\prime}$ or $\gamma_{i}^{\prime \prime}$ to themselves. It must therefore interchange $\gamma_{i}^{\prime}$ and $\gamma_{i}^{\prime \prime}$ for each $i$. This implies that $p=2$.

Corollary 10.4. Almost any flat surface $S$ from the hyperelliptic connected component $\mathcal{H}_{1}^{\text {hyp }}(g-1, g-1)$ has no saddle connections of multiplicity greater then two.

10.3. Constants for the Components of the Stratum $\mathcal{H}_{1}(g-1, g-1)$. We start with the stratum $\mathcal{H}_{1}(g-1, g-1)$ which is exceptional: it has the hyperelliptic connected component.

10.3.1. Hyperelliptic Connected Component $\mathcal{H}_{1}^{\text {hyp }}(g-1, g-1)$. The admissible assignments for the surfaces from the hyperelliptic connected component $\mathcal{H}_{1}^{\text {hyp }}(g-$ $1, g-1)$ are described by Lemma 10.3 The possible multiplicities are one and two. In multiplicity one modifying (18) in accordance with Lemma 10.3 we get the following formula. 
Formula 10.1. The constant for the number of saddle connections of multiplicity one joining distinct zeroes in $\mathcal{H}_{1}^{\text {hyp }}(g-1, g-1)$ is given by

$$
c=(2 g-1) \cdot \frac{\operatorname{Vol}\left(\mathcal{H}_{1}^{h y p}(2 g-2)\right)}{\operatorname{Vol}\left(\mathcal{H}_{1}^{\text {hyp }}(g-1, g-1)\right)}
$$

In other words, collapsing a saddle connection of multiplicity one on a surface $S \in$ $\mathcal{H}_{1}^{\text {hyp }}(g-1, g-1)$ we get a surface $S^{\prime}$ from the hyperelliptic connected component $\mathcal{H}_{1}^{\text {hyp }}(2 g-2)$. Breaking up the zero of a surface $S^{\prime} \in \mathcal{H}_{1}^{\text {hyp }}(2 g-2)$ we necessarily get a surface $S$ from the hyperelliptic component $\mathcal{H}_{1}^{\text {hyp }}(g-1, g-1)$.

TABLE 5. Hyperelliptic component $\mathcal{H}_{1}^{\text {hyp }}(g-1, g-1)$; values of the constants for saddle connections of multiplicity one joining distinct zeroes

\begin{tabular}{|l|c|c|c|c|c|c|c|c|}
\hline & $g=2$ & $g=3$ & $g=4$ & $g=5$ & $g=6$ & $g=7$ & $g=8$ & $g=9$ \\
\hline$c=$ & $\frac{27}{8}$ & $\frac{225}{32}$ & $\frac{6125}{512}$ & $\frac{297675}{16384}$ & $\frac{3361743}{131072}$ & $\frac{9018009}{262144}$ & $\frac{372683025}{8388608}$ & $\frac{59836330125}{1073741824}$ \\
\hline$c \approx$ & 3.375 & 7.03125 & 11.9629 & 18.1686 & 25.6481 & 34.401 & 44.4273 & 55.7269 \\
\hline
\end{tabular}

Example 10.5. Component $\mathcal{H}_{\mathbf{1}}^{\text {hyp }}(\mathbf{1}, \mathbf{1})$. In genus $g=2$ the component $\mathcal{H}_{1}^{\text {hyp }}(1,1)$ coincides with the whole principal stratum $\mathcal{H}_{1}(1,1)$. The formula above gives the same constant as formula 8.3 for the principal stratum in genus $g=2$ :

$$
c=3 \cdot \frac{\operatorname{Vol}\left(\mathcal{H}_{1}^{\text {hyp }}(2)\right)}{\operatorname{Vol}\left(\mathcal{H}_{1}^{\text {hyp }}(1,1)\right)}=3 \cdot \frac{\operatorname{Vol}\left(\mathcal{H}_{1}(2)\right)}{\operatorname{Vol}\left(\mathcal{H}_{1}(1,1)\right)}=\frac{27}{8}=3.375
$$

Example 10.6. Component $\mathcal{H}_{\mathbf{1}}^{\text {hyp }}(\mathbf{2}, \mathbf{2})$. In genus 3 we have the connected component $\mathcal{H}_{1}^{\text {hyp }}(2,2)$. After collapsing zeroes we obtain a hyperelliptic surface $S^{\prime} \in$ $\mathcal{H}_{1}^{\text {hyp }}(4)$. We get

$$
c=5 \cdot \frac{\operatorname{Vol}\left(\mathcal{H}_{1}^{\text {hyp }}(4)\right)}{\operatorname{Vol}\left(\mathcal{H}_{1}^{\text {hyp }}(2,2)\right)}=\frac{225}{32} \approx 7.03
$$

We present more values in table 5

Consider now multiple saddle connections for a flat surface $S$ from the connected component $\mathcal{H}_{1}^{\text {hyp }}(g-1, g-1)$. By Lemma 10.3 we only have $p=2$ in the higher multiplicity, and $S_{i}^{\prime} \in \mathcal{H}^{h y p}\left(2 g_{i}-2\right), i=1,2$, where $g_{1}+g_{2}=g$. Here we always have the $\gamma \rightarrow-\gamma$ symmetry, so $\left|\Gamma_{-}\right|=2$. When $g_{1}=g_{2}$ we also have the stratum interchange symmetry, so $|\Gamma|=2$, and $|\Gamma|=1$ otherwise. Thus, after appropriate modification of formula (23) we get the following constant

Formula 10.2. The constant in multiplicity 2 for saddle connections joining distinct zeroes in $\mathcal{H}_{1}^{\text {hyp }}(g-1, g-1)$ is given by

$$
c=\frac{\left(2 g_{1}-1\right)\left(2 g_{2}-1\right)}{2|\Gamma|} \cdot \frac{\left(2 g_{1}-1\right) !\left(2 g_{2}-1\right) !}{(2 g-1) !} \cdot \frac{\operatorname{Vol}\left(\mathcal{H}_{1}^{\text {hyp }}\left(2 g_{1}-2\right)\right) \operatorname{Vol}\left(\mathcal{H}_{1}^{\text {hyp }}\left(2 g_{2}-2\right)\right)}{\operatorname{Vol}\left(\mathcal{H}_{1}^{\text {hyp }}(g-1, g-1)\right)}
$$


Example 10.7. Component $\mathcal{H}_{\mathbf{1}}^{\text {hyp }}(\mathbf{1}, \mathbf{1})$. Saddle connections of multiplicity two joining distinct zeroes on a surface from $\mathcal{H}_{1}^{\text {hyp }}(1,1)=\mathcal{H}_{1}(1,1)$ were actually, already considered in the section treating the principal stratum. Here after decomposition we get a pair of tori $S_{1}^{\prime}, S_{2}^{\prime}$ each with a marked point. We get the following value for the corresponding constant:

$$
c=\frac{1 \cdot 1}{2 \cdot 2} \cdot \frac{1 ! \cdot 1 !}{3 !} \cdot \frac{\operatorname{Vol}\left(\mathcal{H}_{1}(0)\right) \operatorname{Vol}\left(\mathcal{H}_{1}(0)\right)}{\operatorname{Vol}\left(\mathcal{H}_{1}(1,1)\right)}=\frac{5}{8}=0.625
$$

TABLE 6. Hyperelliptic component $\mathcal{H}_{1}^{\text {hyp }}(g-1, g-1)$; values of the constants for saddle connections of multiplicity two joining distinct zeroes

\begin{tabular}{|l|c|c|c|c|c|c|}
\hline & $g_{2}=1$ & $g_{2}=2$ & $g_{2}=3$ & $g_{2}=4$ & $g_{2}=5$ & $g_{2}=6$ \\
\hline$g_{1}=1$ & $\frac{5}{8}$ & $\frac{63}{32}$ & $\frac{375}{128}$ & $\frac{67375}{16384}$ & $\frac{361179}{65536}$ & $\frac{1867635}{262144}$ \\
\hline$g_{1}=2$ & $\frac{63}{32}$ & $\frac{567}{512}$ & $\frac{22275}{8192}$ & $\frac{441441}{131072}$ & $\frac{1083537}{262144}$ & $\frac{10496871}{2097152}$ \\
\hline$g_{1}=3$ & $\frac{375}{128}$ & $\frac{22275}{8192}$ & $\frac{96525}{65536}$ & $\frac{875875}{262144}$ & $\frac{4027725}{1048576}$ & $\frac{1187146125}{268435456}$ \\
\hline$g_{1}=4$ & $\frac{67375}{16384}$ & $\frac{441441}{131072}$ & $\frac{875875}{262144}$ & $\frac{14889875}{8388608}$ & $\frac{2083228875}{536870912}$ & $\frac{18432519875}{4294967296}$ \\
\hline$g_{1}=5$ & $\frac{361179}{65536}$ & $\frac{1083537}{262144}$ & $\frac{4027725}{1048576}$ & $\frac{2083228875}{536870912}$ & $\frac{8749561275}{4294967296}$ & $\frac{74923166241}{17179869184}$ \\
\hline$g_{1}=6$ & $\frac{1867635}{262144}$ & $\frac{10496871}{2097152}$ & $\frac{1187146125}{268435456}$ & $\frac{18432519875}{4294967296}$ & $\frac{74923166241}{17179869184}$ & $\frac{624359718675}{274877906944}$ \\
\hline
\end{tabular}

Example 10.8. Component $\mathcal{H}_{\mathbf{1}}^{\text {hyp }}(\mathbf{2}, \mathbf{2})$. Consider saddle connections of multiplicity two joining distinct zeroes on a surface from $\mathcal{H}_{1}^{h y p}(2,2)$. After decomposition we get a surface $S_{1}^{\prime} \in \mathcal{H}_{1}(2)$ of genus 2 with a single zero of order 2 and a torus $S_{2}^{\prime} \in \mathcal{H}(0)$ with a marked point. The cone angle between $\gamma_{1}$ and $\gamma_{2}$ is the same at $z_{1}$ and $z_{2}$; it equals $2 \pi$. The cone angle between $\gamma_{2}$ and $\gamma_{1}$ is also the same at $z_{1}$ and $z_{2}$, but now it equals $4 \pi$. The constant is given by

$$
c=\frac{3 \cdot 1}{2 \cdot 1} \cdot \frac{3 ! \cdot 1 !}{5 !} \cdot \frac{\operatorname{Vol}\left(\mathcal{H}_{1}(2)\right) \operatorname{Vol}\left(\mathcal{H}_{1}(0)\right)}{\operatorname{Vol}\left(\mathcal{H}_{1}^{\text {hyp }}(2,2)\right)}=\frac{63}{32} \approx 1.97
$$

We present more values in table 6 and in table 9 in the Appendix.

10.3.2. Connected Component $\mathcal{H}_{1}^{\text {nonhyp }}(g-1, g-1)$; Even Genus. For $g \geq 4, g$ even, the stratum $\mathcal{H}_{1}(g-1, g-1)$ has exactly two connected components: $\mathcal{H}_{1}^{\text {hyp }}(g-$ $1, g-1)$ and $\mathcal{H}_{1}^{\text {nonhyp }}(g-1, g-1)$. Thus, dealing with the connected component $\mathcal{H}_{1}^{\text {nonhyp }}(g-1, g-1)$ we have to exclude all the assignments producing surfaces $S$ in the stratum $\mathcal{H}_{1}(g-1, g-1)$ those that actually belong to the component $\mathcal{H}_{1}^{\text {hyp }}(g-1, g-1)$. The latter problem has already been solved. Thus we get the following answers.

Formula 10.3. For any even genus $g \geq 4$ any flat surfaces $S$ from the connected component $\mathcal{H}_{1}^{\text {nonhyp }}(g-1, g-1)$ having a saddle connection of multiplicity one 
joining distinct zeroes may be obtained from the corresponding surface $S^{\prime}$ from one of the components $\mathcal{H}_{1}^{\text {even }}(2 g-2), \mathcal{H}_{1}^{\text {odd }}(2 g-2)$.

The corresponding constant is equal to

$$
\begin{aligned}
c=(2 g-1) \cdot \frac{\operatorname{Vol}\left(\mathcal{H}_{1}^{\text {even }}(2 g-2)\right)+\operatorname{Vol}\left(\mathcal{H}_{1}^{\text {odd }}(2 g-2)\right)}{\operatorname{Vol}\left(\mathcal{H}_{1}^{\text {nonhyp }}(g-1, g-1)\right)}= \\
=(2 g-1) \cdot \frac{\operatorname{Vol}\left(\mathcal{H}_{1}(2 g-2)\right)-\operatorname{Vol}\left(\mathcal{H}_{1}^{\text {hyp }}(2 g-2)\right)}{\operatorname{Vol}\left(\mathcal{H}_{1}(g-1, g-1)\right)-\operatorname{Vol}\left(\mathcal{H}_{1}^{\text {hyp }}(g-1, g-1)\right)}
\end{aligned}
$$

We proceed with multiplicity two. Here we get the formula analogous to formula 10.2 for the component $\mathcal{H}_{1}^{h y p}(g-1, g-1)$.

Formula 10.4. The constant for multiplicity 2 joining two zeroes in the stratum $\mathcal{H}^{\text {nonhyp }}(g-1, g-1)$ is given by

$$
\begin{aligned}
& c=\frac{\left(2 g_{1}-1\right)\left(2 g_{2}-1\right)}{2|\Gamma|} \cdot \frac{\left(2 g_{1}-1\right) !\left(2 g_{2}-1\right) !}{(2 g-1) !} . \\
& \cdot \frac{\operatorname{Vol}\left(\mathcal{H}_{1}\left(2 g_{1}-2\right)\right) \operatorname{Vol}\left(\mathcal{H}_{1}\left(2 g_{2}-2\right)\right)-\operatorname{Vol}\left(\mathcal{H}_{1}^{\text {hyp }}\left(2 g_{1}-2\right)\right) \operatorname{Vol}\left(\mathcal{H}_{1}^{\text {hyp }}\left(2 g_{2}-2\right)\right)}{\operatorname{Vol}\left(\mathcal{H}_{1}^{\text {nonhyp }}(g-1, g-1)\right)},
\end{aligned}
$$

For multiplicity $p \geq 3$ it is easy to account for the impact of the hyperelliptic component (see Corollary 10.4). We get the following particular case of formula(23) in the case of $\mathcal{H}^{\text {nonhyp }}(g-1, g-1)$

\section{Formula 10.5.}

$$
c=\frac{1}{2|\Gamma|} \cdot \prod_{i=1}^{p}\left(2 g_{i}-1\right) \cdot \frac{\prod_{i=1}^{p}\left(2 g_{i}-1\right) !}{(2 g-1) !} \cdot \frac{\prod_{i=1}^{p} \operatorname{Vol}\left(\mathcal{H}_{1}\left(2 g_{i}-2\right)\right)}{\operatorname{Vol}\left(\mathcal{H}_{1}^{\text {nonhyp }}(g-1, g-1)\right)}
$$

10.3.3. Components $\mathcal{H}^{\text {even }}(g-1, g-1)$ and $\mathcal{H}^{\text {odd }}(g-1, g-1)$. We consider the multiplicity one case first. Assume that $S$ belongs to one of the nonhyperelliptic components $\mathcal{H}^{\text {even }}(g-1, g-1)$ or $\mathcal{H}^{\text {odd }}(g-1, g-1)$. Contracting the saddle connection joining the two zeroes, we merge the two zeroes into one and we get a surface $S^{\prime}$ in $\mathcal{H}^{\text {even }}(2 g-2)$ or $\mathcal{H}^{\text {odd }}(2 g-2)$ correspondingly. Conversely breaking up a single zero of a surface $S^{\prime}$ from a nonhyperelliptic connected component $\mathcal{H}^{\text {even }}(2 g-2)$ or $\mathcal{H}^{\text {odd }}(2 g-2)$ into two zeroes of degrees $g-1$ we get a surface from the component $\mathcal{H}^{\text {even }}(g-1, g-1)$ or $\mathcal{H}^{\text {odd }}(g-1, g-1)$ correspondingly. Thus we have to modify formula (18) in the following way.

Formula 10.6. The constant is equal to

$$
\begin{gathered}
c=(2 g-1) \cdot \frac{\operatorname{Vol}\left(\mathcal{H}_{1}^{\text {even }}(2 g-2)\right)}{\operatorname{Vol}\left(\mathcal{H}_{1}^{\text {even }}(g-1, g-1)\right)} \\
c=(2 g-1) \cdot \frac{\operatorname{Vol}\left(\mathcal{H}_{1}^{\text {odd }}(2 g-2)\right)}{\operatorname{Vol}\left(\mathcal{H}_{1}^{\text {odd }}(g-1, g-1)\right)}
\end{gathered}
$$


Example 10.9. Component $\mathcal{H}^{\text {odd }}(\mathbf{2}, \mathbf{2})$. After collapsing zeroes we obtain a surface $S^{\prime} \in \mathcal{H}^{\text {odd }}(4)$. We get

$$
c=5 \cdot \frac{\operatorname{Vol}\left(\mathcal{H}_{1}^{\text {odd }}(4)\right)}{\operatorname{Vol}\left(\mathcal{H}_{1}^{\text {odd }}(2,2)\right)}=\frac{80}{9} \approx 8.89
$$

Remark 10.10. Note that the similar nonhyperelliptic connected components with even parity of the spins structure do not exist in genus $g=3$.

Now we consider higher multiplicity. We again have to consider multiplicity two separately because some assignments in multiplicity two produce surfaces from the hyperelliptic connected component $\mathcal{H}^{h y p}(g-1, g-1)$, see Lemma 10.1 We again get the formula analogous to formula 10.2 for the component $\mathcal{H}^{\text {hyp }}(g-1, g-1)$ and to formula 10.4 for the component $\mathcal{H}^{\text {nonhyp }}(g-1, g-1)$.

It will be convenient to introduce the following notation. We introduce a function $\delta(\alpha, \phi)$ which has values 0 or 1 . It is equal to 1 when all of the following three conditions are satisfied: all the zeroes of $\alpha$ (if any) have even degrees; $\mathcal{H}(\alpha)$ contains the hyperelliptic component $\mathcal{H}^{\text {hyp }}(\alpha)$; the parity of the spin structure of $\mathcal{H}^{\text {hyp }}(\alpha)$ coincides with $\phi$. Otherwise $\delta(\alpha, \phi)=0$.

Formula (8) for the parity of the spin structure of $\mathcal{H}^{\text {hyp }}\left(\alpha^{\prime}\right)$ shows that

$$
\delta(\alpha, \phi)= \begin{cases}1 & \text { when } \alpha=(2 g-2) \text { and }\left[\frac{g+1}{2}\right]=\phi(\bmod 2) \\ 1 & \text { when } \alpha=(g-1, g-1), g \text { is odd }, \\ & \text { and }\left(\frac{g+1}{2}\right)=\phi(\bmod 2) \\ 0 & \text { otherwise }\end{cases}
$$

We shall also use the following convention: the volume of a nonexistent component is equal to zero. In this notation we get the following six "dummy" volumes for the strata in small genera:

$$
\begin{array}{lcrl}
\operatorname{Vol}\left(\mathcal{H}_{1}^{\text {odd }}(0)\right)=0 & \operatorname{Vol}\left(\mathcal{H}_{1}^{\text {odd }}(2)\right)=0 & \operatorname{Vol}\left(\mathcal{H}_{1}^{\text {even }}(4)\right)=0 \\
\operatorname{Vol}\left(\mathcal{H}_{1}^{\text {even }}(0)\right)=0 & \operatorname{Vol}\left(\mathcal{H}_{1}^{\text {even }}(2)\right)=0 & \operatorname{Vol}\left(\mathcal{H}_{1}^{\text {even }}(2,2)\right)=0
\end{array}
$$

and also we have

$$
\operatorname{Vol}\left(\mathcal{H}^{\text {hyp }}(\alpha)\right)=0 \quad \text { if } \alpha \neq(2 g-2),(g-1, g-1)
$$

Formula 10.7. A surface $S$ from one of the nonhyperelliptic connected components $\mathcal{H}^{\text {even }}(g-1, g-1)$ or $\mathcal{H}^{\text {odd }}(g-1, g-1)$ may have a saddle connection of multiplicity two joining the two distinct zeroes if and only if it can be obtained from surfaces $S_{1}^{\prime}$ and $S_{2}^{\prime}$ of genus $g_{1}, g_{2}$ where $g_{1}+g_{2}=g$ and where the following additional requirements are satisfied. At least one of the surfaces $S_{1}^{\prime}, S_{2}^{\prime}$ does not belong to the hyperelliptic component $\mathcal{H}^{\text {hyp }}\left(2 g_{i}-2\right), i=1,2$; the parities of the spin structures of the surfaces satisfy relation (24) : $\phi\left(S_{1}^{\prime}\right)+\phi\left(S_{2}^{\prime}\right)=\phi(S)$. For $S \in \mathcal{H}^{\text {odd }}(g-1, g-1)$ the spin structures of $S_{1}^{\prime}, S_{2}^{\prime}$ should have opposite parities. This means in particular that there is no longer a stratum interchange symmetry. For $g_{1} \neq g_{2}$ 


$$
\begin{aligned}
c= & \frac{\left(2 g_{1}-1\right)\left(2 g_{2}-1\right)}{2} \cdot \frac{\left(2 g_{1}-1\right) !\left(2 g_{2}-1\right) !}{(2 g-1) !} \cdot \frac{1}{\operatorname{Vol}\left(\mathcal{H}_{1}^{\text {odd }}(g-1, g-1)\right)} \cdot \\
(( & \left(\operatorname{Vol}\left(\mathcal{H}_{1}^{\text {even }}\left(2 g_{1}-2\right)\right)+\delta\left(\left(2 g_{1}-2\right), \text { even }\right) \operatorname{Vol}\left(\mathcal{H}_{1}^{\text {hyp }}\left(2 g_{1}-2\right)\right)\right) \cdot \\
& \left.\cdot\left(\operatorname{Vol}\left(\mathcal{H}_{1}^{\text {odd }}\left(2 g_{2}-2\right)\right)+\delta\left(\left(2 g_{2}-2\right), \text { odd }\right) \operatorname{Vol}\left(\mathcal{H}_{1}^{\text {hyp }}\left(2 g_{2}-2\right)\right)\right)\right)+ \\
& +\left(\left(\operatorname{Vol}\left(\mathcal{H}_{1}^{\text {odd }}\left(2 g_{1}-2\right)\right)+\delta\left(\left(2 g_{1}-2\right), \text { odd }\right) \operatorname{Vol}\left(\mathcal{H}_{1}^{\text {hyp }}\left(2 g_{1}-2\right)\right)\right) \cdot\right. \\
& \left.\cdot\left(\operatorname{Vol}\left(\mathcal{H}_{1}^{\text {even }}\left(2 g_{2}-2\right)\right)+\delta\left(\left(2 g_{2}-2\right), \text { even }\right) \operatorname{Vol}\left(\mathcal{H}_{1}^{\text {hyp }}\left(2 g_{2}-2\right)\right)\right)\right)- \\
- & \delta\left(\left(2 g_{1}-2\right), \text { even }\right) \delta\left(\left(2 g_{2}-2\right), \text { odd }\right) \operatorname{Vol}\left(\mathcal{H}_{1}^{\text {hyp }}\left(2 g_{1}-2\right)\right) \operatorname{Vol}\left(\mathcal{H}_{1}^{\text {hyp }}\left(2 g_{2}-2\right)\right)- \\
- & \left.\delta\left(\left(2 g_{1}-2\right), \text { odd }\right) \delta\left(\left(2 g_{2}-2\right), \text { even }\right) \operatorname{Vol}\left(\mathcal{H}_{1}^{\text {hyp }}\left(2 g_{1}-2\right)\right) \operatorname{Vol}\left(\mathcal{H}_{1}^{\text {hyp }}\left(2 g_{2}-2\right)\right)\right)
\end{aligned}
$$

For $g_{1}=g_{2}=g / 2$, where $g$ is even, we get

$$
\begin{aligned}
& c=\frac{\left(2 g_{1}-1\right)^{2}}{2} \cdot \frac{\left(\left(2 g_{1}-1\right) !\right)^{2}}{(2 g-1) !} \cdot \frac{1}{\operatorname{Vol}\left(\mathcal{H}_{1}^{\text {odd }}(g-1, g-1)\right)} \cdot \\
& \cdot\left(\operatorname{Vol}\left(\mathcal{H}_{1}^{\text {even }}\left(2 g_{1}-2\right)\right)+\delta\left(\left(2 g_{1}-2\right), \text { even }\right) \operatorname{Vol}\left(\mathcal{H}_{1}^{\text {hyp }}\left(2 g_{1}-2\right)\right)\right) \cdot \\
& \cdot\left(\operatorname{Vol}\left(\mathcal{H}_{1}^{\text {odd }}\left(2 g_{2}-2\right)\right)+\delta\left(\left(2 g_{2}-2\right), \text { odd }\right) \operatorname{Vol}\left(\mathcal{H}_{1}^{\text {hyp }}\left(2 g_{2}-2\right)\right)\right)
\end{aligned}
$$

Example 10.11. Component $\mathcal{H}^{\text {odd }}(\mathbf{2}, \mathbf{2})$. From Formula 10.7 we know that the degeneration of multiplicity 2 of a surface from $\mathcal{H}(2,2)$ has the form $(\overline{0}+\overline{0}) \succ$ $(\overline{1}+\overline{1}) \succ$. Since the strata $\mathcal{H}(0)$ and $\mathcal{H}(2)$ are (hyper)elliptic the resulting surface in $\mathcal{H}(2,2)$ is also hyperelliptic, see Lemma 10.3 Hence degeneration of multiplicity 2 is not realizable for flat surfaces from $\mathcal{H}^{\text {odd }}(2,2)$.

The expression for the constant for the surfaces from $\mathcal{H}^{\text {even }}(g-1, g-1)$ is analogous to the one above with the only exception that now we get the stratum interchange symmetry $|\Gamma|=2$ for $g_{1}=g_{2}$ :

$$
\begin{aligned}
& c= \frac{\left(2 g_{1}-1\right)\left(2 g_{2}-1\right)}{2|\Gamma|} \cdot \frac{\left(2 g_{1}-1\right) !\left(2 g_{2}-1\right) !}{(2 g-1) !} \cdot \frac{1}{\operatorname{Vol}\left(\mathcal{H}_{1}^{\text {even }}(g-1, g-1)\right)} \cdot \\
&\left(\left(\left(\operatorname{Vol}\left(\mathcal{H}_{1}^{\text {even }}\left(2 g_{1}-2\right)\right)+\delta\left(\left(2 g_{1}-2\right), \text { even }\right) \operatorname{Vol}\left(\mathcal{H}_{1}^{\text {hyp }}\left(2 g_{1}-2\right)\right)\right) \cdot\right.\right. \\
&\left.\quad \cdot\left(\operatorname{Vol}\left(\mathcal{H}_{1}^{\text {even }}\left(2 g_{2}-2\right)\right)+\delta\left(\left(2 g_{2}-2\right), \text { even }\right) \operatorname{Vol}\left(\mathcal{H}_{1}^{\text {hyp }}\left(2 g_{2}-2\right)\right)\right)\right)+ \\
& \quad+\left(\left(\operatorname{Vol}\left(\mathcal{H}_{1}^{\text {odd }}\left(2 g_{1}-2\right)\right)+\delta\left(\left(2 g_{1}-2\right), \text { odd }\right) \operatorname{Vol}\left(\mathcal{H}_{1}^{\text {hyp }}\left(2 g_{1}-2\right)\right)\right) \cdot\right. \\
&\left.\quad \cdot\left(\operatorname{Vol}\left(\mathcal{H}_{1}^{\text {odd }}\left(2 g_{2}-2\right)\right)+\delta\left(\left(2 g_{2}-2\right), \text { odd }\right) \operatorname{Vol}\left(\mathcal{H}_{1}^{\text {hyp }}\left(2 g_{2}-2\right)\right)\right)\right)- \\
&- \delta\left(\left(2 g_{1}-2\right), \text { even }\right) \delta\left(\left(2 g_{2}-2\right), \text { even }\right) \operatorname{Vol}\left(\mathcal{H}_{1}^{\text {hyp }}\left(2 g_{1}-2\right)\right) \operatorname{Vol}\left(\mathcal{H}_{1}^{\text {hyp }}\left(2 g_{2}-2\right)\right)- \\
&-\left.\delta\left(\left(2 g_{1}-2\right), \text { odd }\right) \delta\left(\left(2 g_{2}-2\right), \text { odd }\right) \operatorname{Vol}\left(\mathcal{H}_{1}^{\text {hyp }}\left(2 g_{1}-2\right)\right) \operatorname{Vol}\left(\mathcal{H}_{1}^{\text {hyp }}\left(2 g_{2}-2\right)\right)\right)
\end{aligned}
$$


Finally, for from multiplicity at least three it is easy to account for the impact of the hyperelliptic component (see Corollary 10.4). We get the following particular case of formula (23):

Formula 10.8. Almost every surface in $\mathcal{H}^{\text {even }}(g-1, g-1)$ or in $\mathcal{H}^{\text {odd }}(g-1, g-1)$ having saddle connections of multiplicity $p \geq 3$ joining two zeroes can be obtained by assignment

$$
\left.\left(\overline{\left(g_{1}-1\right)}\right)+\overline{\left(g_{1}-1\right)}\right) \succ \ldots\left(\overline{\left(g_{p}-1\right)}+\overline{\left(g_{p}-1\right)}\right) \succ
$$

where $g_{i} \geq 1, i=1, \ldots, p$, and $g_{1}+\cdots+g_{p}=g$. The corresponding constant equals

$$
\begin{aligned}
& c=\frac{1}{|\Gamma|} \cdot \prod_{i=1}^{p}\left(2 g_{i}-1\right) \cdot \frac{1}{2^{p-1}} \cdot \frac{\prod_{i=1}^{p}\left(2 g_{i}-1\right) !}{(2 g-1) !} \cdot \frac{1}{\operatorname{Vol}\left(\mathcal{H}_{1}^{\phi}(g-1, g-1)\right)} \cdot \\
& \qquad \sum_{\substack{\phi_{1}, \ldots, \phi_{p} \in\{\text { even }, \text { odd }\} \\
\phi_{1}+\cdots+\phi_{p}=\phi(\bmod 2)}}^{p} \prod_{i=1}^{p}\left(\operatorname{Vol}\left(\mathcal{H}_{1}^{\phi_{i}}\left(2 g_{i}-2\right)\right)+\delta\left(\left(2 g_{i}-2\right), \phi_{i}\right) \operatorname{Vol}\left(\mathcal{H}_{1}^{h y p}\left(2 g_{i}-2\right)\right)\right)
\end{aligned}
$$

Note that here we always have the $\gamma \rightarrow-\gamma$ symmetry, $\left|\Gamma_{-}\right|=2$, which is cancelled by $o(m) !=o(g-1) !($ see (23) $)$. Note that in this particular case we prefer to use summation over all combinations of parities $\phi_{i}$ producing the proper parity of the sum. Thus the stratum interchange symmetry $\Gamma$ depends only on the collection of $g_{i}$, and on their cyclic order.

Example 10.12. Component $\mathcal{H}^{\text {odd }}(\mathbf{2}, \mathbf{2})$. Consider a saddle connection joining the two zeroes of a surface $S \in \mathcal{H}^{o d d}(2,2)$, and suppose that the saddle connection has multiplicity 3 . Each of $S_{i}^{\prime}$, for $i=1,2,3$ is a torus with a marked point. The angle between any consecutive saddle connections at $z_{1}$ and $z_{2}$ equals $2 \pi$. There is a symmetry of order 3 coming from stratum interchange, so $|\Gamma|=3$. Taking into consideration $1 / 4$ from $1 / 2^{p-1}$ we get

$$
c=\frac{1}{3} \cdot \frac{1}{4} \cdot \frac{1 ! \cdot 1 ! \cdot 1 !}{5 !} \cdot \frac{\left(\operatorname{Vol}\left(\mathcal{H}_{1}(0)\right)\right)^{3}}{\operatorname{Vol}\left(\mathcal{H}_{1}^{\text {odd }}(2,2)\right)}=\frac{1}{9} \approx 0.111
$$

Remark 10.13. Let $g \geq 4$ be odd. We see that the maximal multiplicity of a saddle connection joining the distinct zeroes of a surface from the stratum $\mathcal{H}(g-1, g-1)$ is different for different connected components: it equals 2 for almost all surfaces from $\mathcal{H}^{\text {hyp }}(g-1, g-1)$, it equals $g-1$ for almost all surfaces in $\mathcal{H}^{\text {even }}(g-1, g-1)$; it equals $g$ for almost all surfaces in $\mathcal{H}^{\text {odd }}(g-1, g-1)$.

10.4. Other Strata. The description for all other strata $\mathcal{H}(\alpha)$, where all the entries of $\alpha$ are even, is similar to the one just presented.

Formula 10.9. The constant for the nonhyperelliptic connected component $\mathcal{H}^{\phi}(\alpha)$, $\phi \in\{$ even, odd $\}, \alpha \neq(g-1, g-1)$, is given by 


$$
\begin{aligned}
& c=\frac{1}{\left|\Gamma_{-}\right|} \cdot \frac{1}{|\Gamma|} \cdot \prod_{m \in \alpha}\left(\frac{o(m) !}{\prod_{j=1}^{p} o_{j}(m) !}\right) \cdot \prod_{\substack{j=1 \\
a_{j} \neq 0}}^{p} o_{j}\left(a_{j}\right) \cdot \prod_{j=1}^{p}\left(a_{j}+1\right) \cdot \frac{1}{2^{p-1}} \cdot \frac{\prod_{i=1}^{p}\left(\frac{d_{i}}{2}-1\right) !}{\left(\frac{d}{2}-2\right) !} . \\
& \frac{1}{\operatorname{Vol}\left(\mathcal{H}_{1}^{\phi}(\alpha)\right)} \cdot \sum_{\substack{\phi_{1}^{\prime}, \ldots, \phi_{p}^{\prime} \in\{\text { even, odd }\} \\
\phi_{1}^{\prime}+\cdots+\phi_{p}^{\prime}=\phi(\bmod 2)}}^{p}\left(\operatorname{Vol}\left(\mathcal{H}_{1}^{\phi_{i}}\left(\alpha_{i}^{\prime}\right)\right)+\delta\left(\alpha_{i}^{\prime}, \phi_{i}^{\prime}\right) \operatorname{Vol}\left(\mathcal{H}_{1}^{h y p}\left(\alpha_{i}^{\prime}\right)\right)\right)
\end{aligned}
$$

Example 10.14. Stratum $\mathcal{H}^{\text {even }}(\mathbf{4}, \mathbf{2})$. In multiplicity one, after collapsing zeroes we obtain a surface $S^{\prime} \in \mathcal{H}(6)$, where either of the two components $\mathcal{H}^{\text {hyp }}(6)$ and $\mathcal{H}^{\text {even }}(6)$ having even parity of the spin structure are possible. We get

$$
c=7 \cdot \frac{\left(\operatorname{Vol}\left(\mathcal{H}_{1}^{\text {hyp }}(6)\right)+\operatorname{Vol}\left(\mathcal{H}_{1}^{\text {even }}(6)\right)\right)}{\operatorname{Vol}\left(\mathcal{H}_{1}^{\text {even }}(4,2)\right)}=\frac{253001}{18225} \approx 13.88
$$

In multiplicity two we get two surfaces $S_{1}^{\prime} \in \mathcal{H}\left(\alpha_{1}^{\prime}\right), S_{2}^{\prime} \in \mathcal{H}\left(\alpha_{2}^{\prime}\right)$, where $\alpha_{1}^{\prime}+\alpha_{2}^{\prime}=$ $m_{1}+m_{2}-p=4$.

There are two possibilities. In the first case $\alpha_{1}^{\prime}=\alpha_{2}^{\prime}$. Note that any $S^{\prime} \in \mathcal{H}(2)$ has odd parity of the spin structure, see (8). Thus the parity of the resulting flat structure $S$ is even, see Lemma 10.1

The saddle connections $\gamma_{1}$ and $\gamma_{2}$ partition the cone angle $10 \pi$ at $z_{1}$ in two sectors with angles $6 \pi$ and $4 \pi$; they partition the cone angle $6 \pi$ at $z_{2}$ in two sectors with the angles $4 \pi$ and $2 \pi$. Here $a_{1}^{\prime}=2, a_{1}^{\prime \prime}=0$ while $a_{2}^{\prime}=a_{2}^{\prime \prime}=1$ so that while $\mathcal{H}\left(\alpha_{1}^{\prime}\right)=\mathcal{H}\left(\alpha_{2}^{\prime}\right)=\mathcal{H}(2)$, there is no stratum interchange since $a_{1}^{\prime} \neq a_{2}^{\prime}$. Thus $|\Gamma|=1$. We get

$$
c=9 \cdot \frac{1}{2} \cdot \frac{3 ! \cdot 3 !}{7 !} \cdot \frac{\left(\operatorname{Vol}\left(\mathcal{H}_{1}(2)\right)\right)^{2}}{\operatorname{Vol}\left(\mathcal{H}_{1}^{\text {even }}(4,2)\right)}=\frac{6}{25}=0.24
$$

In the second case after degeneration we get $S_{1}^{\prime} \in \mathcal{H}_{1}(0), S_{2}^{\prime} \in \mathcal{H}_{1}(4)$ Since $\mathcal{H}(0)$ has odd parity of the spin structure, $S_{2}^{\prime} \in \mathcal{H}_{1}^{\text {odd }}(4)$ in order to result in a surface $S \in \mathcal{H}^{\text {even }}(4,2)$ (see Lemma 10.1).

The saddle connections $\gamma_{1}$ and $\gamma_{2}$ partition the cone angle $10 \pi$ at $z_{1}$ in two sectors with the angles $2 \pi$ and $8 \pi$; they partition the cone angle $6 \pi$ at $z_{2}$ in two sectors with the angles $2 \pi$ and $4 \pi$. We get

$$
c=5 \cdot \frac{1}{2} \cdot \frac{1 ! \cdot 5 !}{7 !} \cdot \frac{\operatorname{Vol}\left(\mathcal{H}_{1}(0)\right) \operatorname{Vol}\left(\mathcal{H}_{1}^{\text {odd }}(4)\right)}{\operatorname{Vol}\left(\mathcal{H}_{1}^{\text {even }}(4,2)\right)}=\frac{640}{729} \approx 0.8779
$$

Multiplicity 3 is not realizable in the component $\mathcal{H}^{\text {even }}(4,2)$ (see the next subsection).

Example 10.15. Stratum $\mathcal{H}^{\text {odd }}(\mathbf{4}, \mathbf{2})$. This component is similar in many aspects to the previous one, so we skip those details of calculations which are common for both components.

The first case is $\mathcal{H}^{\text {odd }}(4,2)$ with multiplicity $p=1$. After collapsing zeroes we obtain a surface $S^{\prime} \in \mathcal{H}(6)$, the only component that results is $\mathcal{H}^{\text {odd }}(6)$. We get

$$
c=7 \cdot \frac{\operatorname{Vol}\left(\mathcal{H}_{1}^{\text {odd }}(6)\right)}{\operatorname{Vol}\left(\mathcal{H}_{1}^{\text {odd }}(4,2)\right)}=\frac{147}{10}=14.7
$$


Consider multiplicity 2. The case of degeneration to two surfaces $S_{1}^{\prime}, S_{2}^{\prime} \in \mathcal{H}(2)$ does not take place here, since the surface $S \in \mathcal{H}(4,2)$ obtained from two surfaces from $\mathcal{H}(2)$ has even parity of spin structure.

Thus, the only possibility is $S_{1}^{\prime} \in \mathcal{H}_{1}(0) ; S_{2}^{\prime} \in \mathcal{H}_{1}(4), a_{2}^{\prime}=3, a_{2}^{\prime \prime}=1$. Since $\mathcal{H}(0)$ has odd parity of the spin structure, $S_{2}^{\prime}$ must be in components of $\mathcal{H}(4)$ having even parity of spin structure in order to result a surface $S \in \mathcal{H}^{\text {odd }}(4,2)$ (see Lemma 10.1). There is only one such component: $\mathcal{H}^{h y p}(4)$.

Thus we get

$$
c=5 \cdot \frac{1}{2} \cdot \frac{1 ! \cdot 5 !}{7 !} \cdot \frac{\operatorname{Vol}\left(\mathcal{H}_{1}(0)\right) \operatorname{Vol}\left(\mathcal{H}_{1}^{\text {hyp }}(4)\right)}{\operatorname{Vol}\left(\mathcal{H}_{1}^{\text {odd }}(4,2)\right)}=\frac{15}{64} \approx 0.2344
$$

(see analogous calculation for the component $\mathcal{H}^{\text {even }}(4,2)$.

Consider now multiplicity 3 . It is not hard to check that $S_{1}^{\prime} \in \mathcal{H}(2), S_{2}^{\prime}, S_{3}^{\prime} \in$ $\mathcal{H}(0)$. Note that both $\mathcal{H}(0)$ and $\mathcal{H}(2)$ have odd parity of the spin structure. Thus the resulting surface $S \in \mathcal{H}(4,2)$ always has odd parity of the spin structure, see Lemma 10.1

The saddle connections $\gamma_{1}, \gamma_{2}, \gamma_{3}$ partition the cone angle $10 \pi$ at $z_{1}$ in three sectors with the angles $6 \pi, 2 \pi$, and $2 \pi$; they partition the cone angle $6 \pi$ at $z_{2}$ in three sectors with the angles $2 \pi$. We find

$$
c=3 \cdot \frac{1}{4} \cdot \frac{3 ! \cdot 1 ! \cdot 1 !}{7 !} \cdot \frac{\operatorname{Vol}(\mathcal{H}(2))\left(\operatorname{Vol}\left(\mathcal{H}_{1}(0)\right)\right)^{2}}{\operatorname{Vol}\left(\mathcal{H}_{1}^{\text {odd }}(4,2)\right)}=\frac{21}{320} \approx 0.06563
$$

\section{Part 2. Saddle Connections Joining a Zero to Itself}

In this part we consider the second problem of this paper: we count closed saddle connections joining a zero to itself.

\section{Approaching the Principal Boundary by Shrinking Closed GEODESICS}

11.1. Configurations of Closed Saddle Connections and Corresponding Surface Decompositions. We have already indicated in Section 3.2 that together with a closed saddle connection $\gamma_{1}$ joining a zero to itself some other saddle connections $\gamma_{2}, \ldots, \gamma_{p}$ homologous to $\gamma_{1}$ might be present on a surface $S$, see Figure 17. There might be also some metric cylinders filled with regular closed geodesics homologous to $\gamma_{1}$; such cylinders are bounded by the singular closed geodesics from the collection $\gamma_{1}, \ldots, \gamma_{p}$. Suppose that the curves $\gamma_{j}$ with indices from a set $J=\left\{i_{1}, \ldots, i_{l}\right\} \subset\{1, \ldots, p\}$ bound $q$ cylinders. The complement of the union of the curves $\gamma_{j}$ and the cylinders splits into $p=m-q$ disjoint surfaces $S_{1}, \ldots, S_{p}$. For example, the surface $S$ presented in Figure 7 is composed of $p=4$ surfaces $S_{j}$ and of $q=2$ cylinders, where one cylinder joins $S_{2}$ to $S_{3}$ and the other cylinder joins $S_{3}$ to $S_{4}$. When $p \geq 2$ the boundary of the closure $\bar{S}_{j}$ of any surface $S_{j} \subset S$ is a union of two singular closed geodesics $\gamma_{j-1} \cup \gamma_{j}$.

The sum of the genera of the $S_{j}$ is $g-1$. The surfaces $S_{j}$ are attached in some cyclic order. Thus, for any $j$ the "next surface" $S_{j+1}$ and the "previous surface" $S_{j-1}$ make sense $\bmod p$. We use the following convention for the cyclic order: 
let $\vec{n}$ be the vector orthogonal to $\vec{\gamma}_{j}$ and pointing from $S_{j}$ to $S_{j+1}$. Then the frame $\left(\vec{n}, \vec{\gamma}_{j}\right)$ corresponds to the canonical orientation of the surface induced by the complex structure.



Figure 7. Homologous closed geodesics of high multiplicity. Topological picture.

We distinguish two types of surfaces $S_{j}$.

If the boundary of the closure $\bar{S}_{k}$ of $S_{k} \subset S$ has two connected components, we get a surface of the first type. In this case the boundary $\partial \bar{S}_{k}$ is formed by two disjoint singular closed geodesics $\gamma^{\prime \prime}=\gamma_{k-1}$ and $\gamma^{\prime}=\gamma_{k}$. The surfaces $S_{1}$ and $S_{2}$ in Figure 7 are of that type. The closed geodesics $\gamma^{\prime \prime}$ contains a single conical point $z_{k}$ the closed geodesics $\gamma^{\prime}$ contains a single conical point $w_{k}$. We denote by $\left(2 b_{k}^{\prime}+3\right) \pi$ the cone angle at $w_{k}$ inside $\bar{S}_{k}$, and by $\left(2 b_{k}^{\prime \prime}+3\right) \pi$ the cone angle at $z_{k}$ inside $\bar{S}_{k}$.

Suppose now that the boundary of the closure $\bar{S}_{k}$ of $S_{k} \subset S$ has a single connected component produced by two singular closed geodesics sharing the same conical point $P$. Cut $\bar{S}_{k}$ at this conical point. If the boundary of the resulting surface now has two connected components, that means that the surface $\bar{S}_{k}$ is obtained from a surface described in the paragraph above by identification of the conical points $w \sim z$, and we do not distinguish a surface of this type from those discussed above.

We also put in the first group the surface $S_{1}$ if $\bar{S}_{1}=S$; that is, if we have a singular closed geodesic $\gamma_{1}$ of multiplicity one which does not bound a cylinder of regular closed geodesics. Cutting $S$ by $\gamma_{1}$ we again obtain a surface with two disjoint boundary components $\gamma^{\prime} \neq \gamma^{\prime \prime}$ as above. The initial unique conical point $P \in \gamma_{1}$ produces two distinct conical points; one on each of the two boundary components.

Consider now the remaining case. Suppose that the boundary of $\bar{S}_{i}$ has a single connected component produced by two singular closed geodesics $\gamma_{i-1}=\gamma^{\prime \prime}$ and $\gamma_{i}=\gamma^{\prime}$ sharing the same conical point $P$. If the boundary of the surface $\bar{S}_{i}$ cut at $P$ still has a single connected component, we say that we get a surface of the second type. For example, the surfaces $S_{3}$ and $S_{4}$ at Figure 7 are of that type. The curves $\gamma^{\prime}, \gamma^{\prime \prime}$ bound angles $2\left(a_{i}^{\prime}+1\right) \pi$ and $2\left(a_{i}^{\prime \prime}+1\right) \pi$ inside $S_{i}$ at the conical point $P$. The two boundary curves $\gamma^{\prime}$ and $\gamma^{\prime \prime}$ are joined together at the zero forming a "figure eight". 
Therefore we get two types of surfaces $S_{k}, S_{i}$; a boundary of each surface is produced by a pair of singular closed geodesics $\gamma^{\prime}, \gamma^{\prime \prime}$. Altogether the configuration consists of the data

$$
\left(J, a_{i}^{\prime}, a_{i}^{\prime \prime}, b_{k}^{\prime}, b_{k}^{\prime \prime}\right)
$$

We will show how to metrically shrink the boundary components of each $S_{i}$ to produce a closed surface. For surfaces of the first type the boundary curves will shrink to zeroes of orders $b_{k}^{\prime}, b_{k}^{\prime \prime}$ and for surfaces of the second type to a single zero of order $a_{i}^{\prime}+a_{i}^{\prime \prime}$. Each such closed surface will then lie in a stratum $\mathcal{H}\left(\alpha_{i}^{\prime}\right)$ and the union of these strata comprises the principal boundary.

The goal of the rest of this section is to describe how, for each $S_{i}$, one can naturally "shrink" the boundary components $\gamma^{\prime}, \gamma^{\text {" }}$ to obtain a closed regular flat surface. We start with surfaces of the second type.

11.2. Shrinking a Pair of Adjacent Holes. Suppose that the boundary of $\bar{S}_{i}$ has a single connected component produced by two singular closed geodesics $\gamma^{\prime}, \gamma^{\prime \prime}$ sharing the same conical point $P$; the boundary of such surface has a form of figure eight. By assumption the surface $S_{i}$ is of the second type, so cutting $\bar{S}_{i}$ at $P$ we get a surface with a connected boundary. The surgery corresponding to cutting $\bar{S}_{i}$ at $P$ is illustrated at Figure [10] We start with the surface presented on the right of Figure 10 (there is an extra flat cylinder drawn in the picture, this flat cylinder will be discussed later) and we get the surface presented in the middle of Figure 10 By construction, the boundary of the surface obtained after the surgery is made up of two parallel geodesic segments of the same length (coming from $\gamma^{\prime}$ and $\gamma^{\prime \prime}$ ) joining two singular points $P_{1}, P_{2}$ (obtained by breaking $P$ into two distinct points). The cone angles inside $S_{i}$ at these points are denoted by $2 \pi\left(a_{i}^{\prime}+1\right)$ and $2 \pi\left(a_{i}^{\prime \prime}+1\right)$. Now we identify the two geodesic segments which form the boundary; see the left picture at Figure 10] We get a closed surface with a saddle connection $\gamma$ joining the two zeroes. By construction this saddle connection has direction $\gamma_{1}$ and length $\left|\gamma_{1}\right|$; moreover, by construction the resulting surface does not have any other saddle connections homologous to $\gamma$. Thus, if the initial surface $S$ belongs to $\mathcal{H}_{1}^{\varepsilon, \text { thick }}(\alpha)$ then the surface obtained from $S_{i}$ by our surgery does not have any other short saddle connections, except $\gamma$. Hence, following the construction of section 8.2 we can collapse the saddle connection $\gamma$ into a single zero of order $a_{i}=a_{i}^{\prime}+a_{i}^{\prime \prime}$. We have proved that for any $S$ from $\mathcal{H}_{1}^{\varepsilon, t h i c k}(\alpha)$ there is a canonical way to associate to every component $S_{i}$ of the second type a closed flat surface $S_{i}^{\prime}$.

In other words, we have proved that for a surface $S_{i}$ of the second type (one having boundary formed by two small adjacent holes) there is a canonical way to shrink metrically the holes, obtaining as a result a closed flat surface $S_{i}^{\prime}$.

11.3. Transporting a Small Hole Along a Flat Surface. Intuitively one can interpret the surgery described in the previous section as annihilation of a "+" and a "-" hole which are joined together. In this section we show how one can continuously deform the location of an isolated small hole on a flat surface. In the next section we consider a flat surface with a pair of small holes located at different places on the surface. Using the hole-transport construction we move "+" and "-" holes in such way that they become adjacent to each other and then we make them annihilate as was described in the previous section.

In this section we consider the case of multiplicity one. Moreover, we assume that the short closed saddle connection $\gamma$ of multiplicity one does not bound a metric 
cylinder. Choose some orientation of $\gamma$; let $\vec{v}=\operatorname{hol}(\gamma)$. Let $\Delta$ be the length of the shortest saddle connection different from $\gamma$. Assume that $\Delta \gg \varepsilon=|\gamma|$. Now cut the surface $S$ along $\gamma$. Since $\gamma$ is not homologous to zero, we get a connected surface $S_{1}$ with boundary having two connected components. Each component is formed by a single geodesic segment containing exactly one conical point. By construction the size $\varepsilon$ of the holes is small with respect to the scale of the surface $S_{1}$, and the holes are located relatively far away one from the other.

Choose one of the holes, say, the positive one for which the orientation induced by the natural orientation of the boundary coincides with the chosen orientation of $\gamma$. We denote this hole as $\gamma_{+}$. Let $P$ be the unique singularity point located on $\gamma_{+}$. Consider the set $D_{\Delta}(P) \subset S_{1}$ of points in $S_{1}$ located at distance less than or equal to $\Delta$ from $P$. Denote the cone angle at $P$ by $(2 b+3) \pi$; it is easy to see that the cone angle is always an odd multiple of $\pi$, with $b$ being some nonnegative integer. The set $D_{\Delta}(P)$ is composed of $2 b+1$ regular sectors, isometric to metric half-discs of radius $\Delta$ and of one irregular sector. The irregular sector can be constructed metrically as follows: superpose two copies of metric half-discs of radius $\Delta$; then shift one with respect to the other by $\varepsilon$ in direction of the common diameter; finally, take the union of the resulting metric half-discs; see Figure 8 The distinguished sector contains the hole, see Figure 8 so its impact to the cone angle at $P$ is $2 \pi$ instead of $\pi$. We choose the boundaries of the sectors to have directions $\pm \vec{v}$. We denote the distinguished sector by $\Omega_{0}$; we enumerate the other sectors $\Omega_{1}, \ldots, \Omega_{2 b+1}$ in a cyclic order.


FiguRE 8. Neighborhood $D_{\Delta}(P)$ of a boundary singularity $P$ is composed of a special sector $\Omega_{0}$ and of $2 b+1$ regular sectors $\Omega_{1}, \ldots \Omega_{2 b+1}$. The cone angle at $P$ is $(2 b+3) \pi$.

Consider a geodesic segment $\tau \subset D_{\Delta}(P)$ having the conical point $P$ as one of the endpoints; let $P^{\prime}$ be the other endpoint of $\tau$; let $\delta$ be the length of $\tau$. We assume that $\delta<\Delta-\varepsilon$, so $P^{\prime}$ is located in the interior of $D_{\Delta}(P)$. Our goal is to "move the hole" to the point $P^{\prime}$. We treat separately the four cases listed below which describe all possible locations of $\tau$. Each time we modify some sectors $\Omega_{i}$ or the identification of the boundaries of the sectors in such way that after regluing of the modified sectors a neighborhood of the boundary of the modified domain $D_{\Delta}^{\prime}(P)$ stays isometric to a neighborhood of the boundary of the initial domain $D_{\Delta}(P)$. In this way we can cut $D_{\Delta}(P)$ out of the flat surface; make a surgery on $D_{\Delta}(P)$ and then metrically paste back the modified domain $D_{\Delta}^{\prime}(P)$ into the surface. Here is a complete list of possible locations of $\tau$ (see also Figure 9); as usual indices are taken modulo the largest one, so $\Omega_{-1}=\Omega_{2 b+1}$. 
I. $\Omega_{i}, i=2, \ldots, 2 b+1$


II.
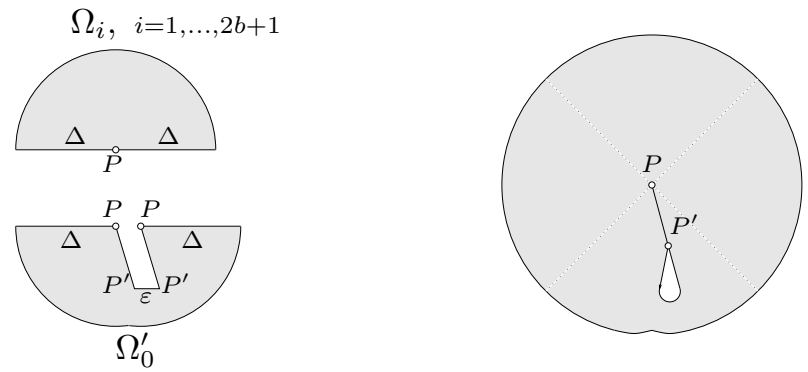

III.
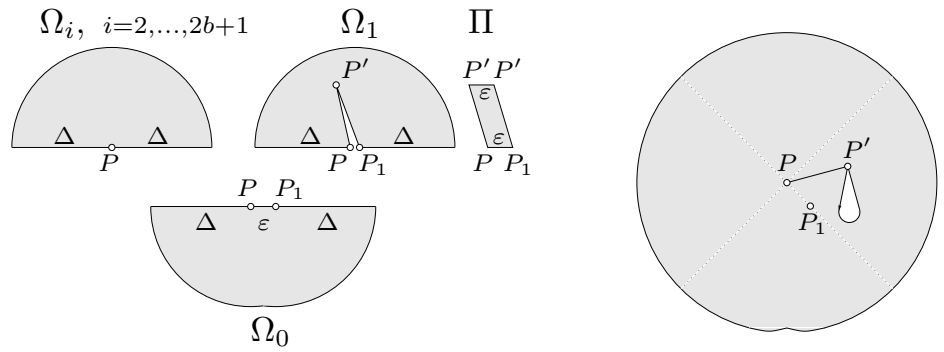

IV.
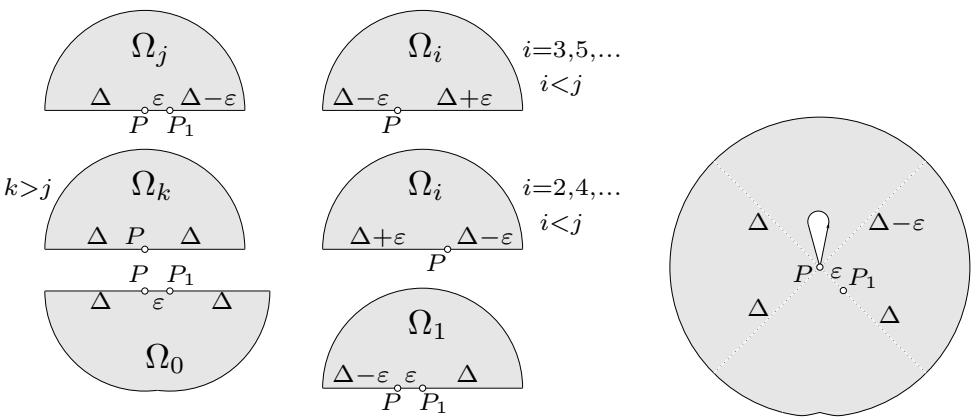

Figure 9. Transporting a hole from $P$ to $P^{\prime}$ along $\tau$ and tunnelling it to a nonadjacent sector.

I. $\tau \subset \Omega_{0}$ and $\tau$ has direction $\pm \vec{v}$;

II. $\tau \subset \Omega_{0}$ and $\tau$ has direction different from $\pm \vec{v}$;

III. $\tau \subset \Omega_{ \pm 1}$ and $\tau$ has direction different from $\pm \vec{v}$;

IV. $\tau \subset \Omega_{i}$ and $i \neq-1,0,1$ 

I. $\tau \subset \Omega_{0}$ and $\tau$ has direction $\pm \vec{v}$;
II. $\tau \subset \Omega_{0}$ and $\tau$ has direction different from $\pm \vec{v}$;
III. $\tau \subset \Omega_{ \pm 1}$ and $\tau$ has direction different from $\pm \vec{v}$;
IV. $\tau \subset \Omega_{i}$ where $i \neq-1,0,1$

\section{Moving the hole along $\vec{v}$.}

To move the hole in direction $\vec{v}$ it is sufficient to modify the identification of the boundary of $\Omega_{0}$ with the boundary of $\Omega_{1}$. Similarly, to move the hole in direction $-\vec{v}$ it is sufficient to modify the identification of the boundary of $\Omega_{0}$ with the boundary of $\Omega_{-1}$. The first case is illustrated at Figure 9 case I. We identify the segments $\left[P, P^{\prime}\right]$ on $\Omega_{0}$ and on $\Omega_{1}$ (this is exactly our geodesic segment $\tau$ of length $\delta$ ). Then we leave a segment of length $\varepsilon$ on $\Omega_{0}$ without identification, but we identify its endpoints getting a single point $P^{\prime}$. This is the new hole. Then we identify the remaining segments of length $\Delta-\delta$ (the ones to the right of the point $P^{\prime}$ at Figure9. case I) on $\Omega_{0}$ and on $\Omega_{1}$.

In other words, our surgery consists of marking a superfluous segment of length $\varepsilon$ on the boundary of $\Omega_{0}$ at the distance $\delta$ to the right (correspondingly to the left) of the initial location.

\section{Moving the hole inside the distinguished sector.}

Consider a parallelogram $\Pi$ in $\Omega_{0}$ having $\gamma$ and $\tau$ as a pair of sides (see Figure 9 II); let $\gamma^{\prime}$ and $\tau^{\prime}$ be the other two sides of $\Pi$ parallel to $\gamma$ and $\tau$ correspondingly. We can think of $\tau^{\prime}$ as of a parallel translation of $\tau$ by $\gamma$. Cut $\Pi$ out of $\Omega_{0}$ and identify the opposite sides $\tau$ and $\tau^{\prime}$ in $\Omega_{0}^{\prime}=\Omega_{0}-\Pi$. Perform the initial identification of the boundaries of the sectors using $\Omega_{0}^{\prime}$ instead of $\Omega_{0}$. As a result of this surgery we get a new domain $D_{\Delta}^{\prime}(P)$ with a hole located at the point $P^{\prime}$. Note that performing this surgery we have reduced the area of the surface by the area of the parallelogram $\Pi$ which was cut out.

III. Moving the hole inside one of the two adjacent sectors $\Omega_{-1}, \Omega_{1}$.

If the segment $\tau$ transversal to the direction $\vec{v}$ is located in one of the sectors $\Omega_{-1}, \Omega_{1}$ we can perform an operation inverse to the one presented above. Suppose that, say, $\tau \subset \Omega_{1}$. Consider a parallelogram $\Pi$ in the Euclidean plane with a pair of sides having the same lengths and directions as $\gamma$ and $\tau$ correspondingly. Slit $\Omega_{1}$ along $\tau$ from $P$ to $P^{\prime}$; let $\tau$ and $\tau^{\prime}$ be the sides of the resulting slit. Identify the corresponding sides of $\Pi$ with $\tau$ and $\tau^{\prime}$. We have modified the sector $\Omega_{1}$ in the following way: the modified sector $\Omega_{1}^{\prime}=\Omega_{1}+\Pi$ has an extra segment $\gamma$ at the base, where $\operatorname{hol}(\gamma)=\vec{v}$, and a new hole $\gamma^{\prime}$ at the point $P^{\prime}$, where $\operatorname{hol}\left(\gamma^{\prime}\right)=\vec{v}$. Perform the initial identification of the boundaries of the sectors using $\Omega_{1}^{\prime}$ instead of $\Omega_{1}$; add a new one identifying the segment $\gamma$ of length $\varepsilon$ at the base of $\Omega_{1}^{\prime}$ with the segment $\gamma$ of length $\varepsilon$ at the base of $\Omega_{0}$. As a result of this surgery we get a new domain $D_{\Delta}^{\prime}(P)$ with a hole located at the point $P^{\prime}$. Note that performing this surgery we have increased the area of the surface by the area of the parallelogram $\Pi$ which was pasted in.

\section{Tunnelling the hole to a nonadjacent sector.}

If the segment $\tau$ is located in a sector $\Omega_{i}$ where $i \neq-1,0,1$ we first "push the hole" either to $\Omega_{i}$ or to its neighbor. This reduces the situation to one of the cases I-III. We push the hole to the sector $\Omega_{j}$, where $j=i$ if $i$ is even and $j=i-1$ if $i$ is odd. To do this we change the identifications of the boundary segments of the 
sectors $\Omega_{0}, \ldots, \Omega_{j}$ as indicated at Figure 9 case IV. The operation of tunnelling the hole to a nonadjacent sector does not change the area of the surface.

11.4. Shrinking a Pair of Nonadjacent Holes. To simplify the construction above we assumed that the path $\tau$, along which we have transported the hole, is a geodesic segment which is shorter than the shortest saddle connection. It is quite clear how to generalize the construction in several ways.

A geodesic segment $\tau$ transversal to $\vec{v}$ can be replaced by a path $\tau$ transversal to $\vec{v}$; the result of the surgery depends only on the homotopy class of $\tau$, where the homotopy is performed with the fixed endpoints; the path remains transversal to $\vec{v}$ during the homotopy; the homotopy is performed inside the surface with punctured singularities.

We can invert our construction: having a hole located close to a zero we can move it to the zero.

The segment (path) $\tau$ need not be necessarily short: an appropriate surgery can be performed using a finite covering of $\tau$ by domains $D_{\Delta}\left(P_{0}\right), D_{\Delta_{1}}\left(P_{1}\right), \ldots, D_{\Delta_{s}}\left(P_{s}\right)$. However, we require that $\tau$ is either a segment parallel to $\vec{v}$ or a segment (path) transversal to $\vec{v}$. We also require that $\tau$ does not have self-intersections, and, moreover, that it does not make "almost loops" when a pair of points located far one from another with respect to parameterization of $\tau$ by length occur to be close to each other on the surface.

Finally, we can consider a situation when $\tau$ is a broken line of geodesic segments joining singularities $P_{0}, P_{1}, \ldots, P_{s}$. We can consecutively move the hole along the first segment $\tau_{01}$ from singularity $P_{0}$ to singularity $P_{1}$; perform if necessary tunnelling of the hole from one sector at $P_{1}$ to another; move the hole along the second segment $\tau_{12}$ from singularity $P_{1}$ to singularity $P_{2}$; etc, and finally bring the hole to the appropriate sector of $P_{s}$.

We can now return to our original problem. We have a surface $S_{1}$ with two small holes which we want to shrink metrically. We move one hole to another and then apply the construction of shrinking a pair of adjacent holes.

Note that the operation of moving a hole from $P_{0}$ to $P_{1}$ typically changes the area of the surface. However, the change of the area is at most the product of the length of circumference of the hole $\gamma$ by the length of the path $\tau$.

\section{Constructing Surfaces with Homologous Closed Saddle Connections}

Our objective now is to reverse the shrinking operation to show how to build homologous saddle connections with associated configuration $\left(J, a_{i}^{\prime}, a_{i}^{\prime \prime}, b_{k}^{\prime}, b_{k}^{\prime \prime}\right)$ out of simpler surfaces. We shall start with a collection of closed surfaces and via a "figure eight" and "creating a pair of holes construction" build a closed surface with a curve or set of homologous curves joining a zero to itself.

Fix $0<r<1 / 4$ and let $\mathcal{F}^{\prime}$ denote a compact simply connected subset of $\mathcal{H}_{1}\left(\sqcup \alpha_{i}^{\prime}\right)$ such that for $S^{\prime} \in \mathcal{F}^{\prime}$ every saddle connection has length at least $2 \varepsilon^{r}$. By Lemma 7.1 its complement has volume $O\left(\varepsilon^{2 r}\right)$. Choose a homology basis $\beta_{i}$ that is valid for all $S^{\prime} \in \mathcal{F}^{\prime}$.

12.1. Figure Eight Construction. Given a surface $S_{i}^{\prime}$, a zero (or a marked point) $z_{i}$ of order $a_{i}$, and a pair of numbers $a_{i}^{\prime}+a_{i}^{\prime \prime}=a_{i}$ we may break up the zero and perform the slit construction. We may then identify the two endpoints of the slit. 

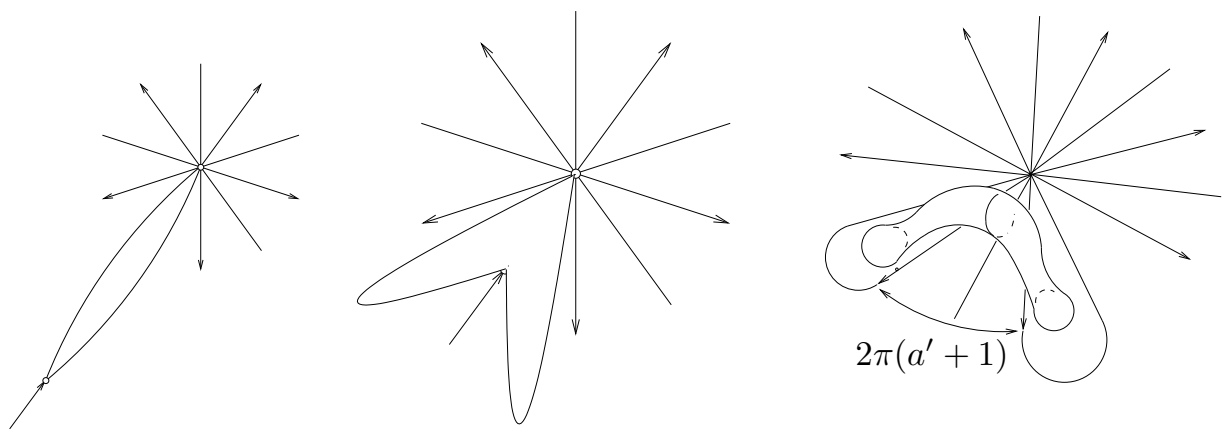

Figure 10. Here we applied the figure eight construction with $a^{\prime}=0$ and $a^{\prime \prime}=4$ to a zero of order $a=a^{\prime}+a^{\prime \prime}=4$, and then we glued in a cylinder. The degree of the resulting zero is 6 .

This gives a surface with two circular boundary components $\gamma_{i}^{\prime}, \gamma_{i}^{\prime \prime}$, which are joined at a point, see Figure 10. The two circular boundary components separate the total angle at the point into angles $2 \pi\left(a_{i}^{\prime}+1\right)$ and $2 \pi\left(a_{i}^{\prime \prime}+1\right)$. By convention on the choice of $\gamma_{i}^{\prime}, \gamma_{i}^{\prime \prime}$ (see Section 9.2) turning around $z_{i}$ in a clockwise direction from $\gamma_{i}^{\prime \prime}$ to $\gamma_{i}^{\prime}$ inside the surface $S_{i}^{\prime}$ we turn by the angle $2 \pi\left(a_{i}^{\prime}+1\right)$.

Recall that we may break the zero of order $a_{i}$ up in $a_{i}+1$ ways. Thus the figure eight construction is not uniquely defined; the construction gives $a_{i}+1$ surfaces.

12.2. Creating a Pair of Holes. In this construction we are given a pair of distinct points and a vector. We shall "open" the surface at the two points using the vector to build a surface with two boundary components. The construction depends on choosing a path between the two points, and so is not quite canonical. Suppose then that $z$ and $w$ are zeroes of orders $b^{\prime}, b^{\prime \prime} \geq 0$ on a surface $S^{\prime}, \gamma$ is a vector of length $\varepsilon$. Let $\rho$ be the shortest path joining $z$ and $w$. It consists of saddle connections. On a set of surfaces of full measure none of the saddle connections are in the direction $\gamma$. The endpoints of the segments are located at conical points. We need first to estimate the length of $\rho$ and establish a few simple properties of the path. We use Corollary 5.7 from paper [MS] (in which we adjusted the notations).

Proposition (H. Masur, J. Smillie). Let $S$ be a closed flat surface with diameter d. Let $\gamma$ be any geodesic segment joining singularities whose length is minimal among all geodesic segments that join singularities. Assume that $\gamma$ is not a simple closed curve which bounds a metric cylinder. Let $\Delta$ be the length of the shortest geodesic element $\beta$ joining singularities not equal to $\gamma$. Then there is a constant $C$ depending only on the genus of the surface $M$ such that $d \leq C / \Delta$.

From the assumption on $\mathcal{F}^{\prime}$, and the above Proposition $|\rho| \leq C \varepsilon^{-r}$ for a new constant $C$. Since $\rho$ is a shortest path it does not have any self-intersections, so a conical point $P_{i}$ may have at most two segments of $\rho$ adjacent to it. If $\rho$ comes within $\varepsilon+\varepsilon^{2 r}$ of a singularity other than at its endpoints, we may modify $\rho$ choosing a broken line going through that singularity, increasing the length of $\rho$ by at most $2\left(\varepsilon+\varepsilon^{2 r}\right)$. Since the shortest loop on the surface has length at least $\varepsilon^{r}$, and there are at most $2 g-2$ conical points, for small $\varepsilon$, after at most $2 g-2$ replacements we can assume that the new path $\rho$ does not pass within $\varepsilon+\varepsilon^{2 r}$ of any singularity except at its endpoints. The total increase in length is at most $2 g\left(\varepsilon+\varepsilon^{2 r}\right)$ and so 
its length is bounded by $C \varepsilon^{-r}$ for a slightly different $C$. (The path might now be not shortest.)

Proposition 12.1. $\quad$ (1) Any line segment of length $\varepsilon+\varepsilon^{2 r}$ in direction $\vec{v}$ through any point on $\rho$ does not meet $\rho$ again.

(2) For small $\varepsilon$ and any saddle connection $\beta$, the algebraic intersection $|\rho \cdot \beta| \leq$ $|\beta| \varepsilon^{-2 r}$.

Proof. To prove (1) notice that if the intersections points $Q_{1}, Q_{2}$ of the line segment with $\rho$ were further than $(2 g+1)\left(\varepsilon+\varepsilon^{2 r}\right)$ apart on $\rho$, we could shorten $\rho$ by more than $2 g\left(\varepsilon+\varepsilon^{2 r}\right)$ following the line segment between $Q_{1}$ and $Q_{2}$ instead of $\rho$, which is impossible. If $Q_{1}, Q_{2}$ are within $2 g\left(\varepsilon+\varepsilon^{2 r}\right)$ of each other on $\rho$ we have a loop of length at most $(2 g+1)\left(\varepsilon+2 \varepsilon^{2 r}\right)<2 \varepsilon^{r}$ for small $\varepsilon$, which is also a contradiction.

The proof of (2) is similar.

Now returning to the problem of creating a pair of holes, suppose we are given a pair of separatrices leaving $z$ and $w$ in direction $\gamma$. The notion of $z^{\prime}$ as the point $z+\gamma$ makes sense for small $\gamma$. Perform the figure eight construction using the points $z$ and $z^{\prime}$ creating a surface with a pair of holes joined at a point $P$.

We can now transport a hole from $P$ to $w$ along $\rho$ as was described in the previous section. Conclusion 1) of the Proposition says that the hole transport is well-defined. We get a surface with two holes. We may then tunnel the hole at $w$ so that it is adjacent to the given separatrix. Note that the operation of creating a pair of holes typically changes the area of the surface. However, the change of the area is at most the product of the length of circumference of the hole $\gamma$ by the length of the path $\rho$. Our estimates show that the change of the area is bounded by $C \varepsilon^{1-r}$.

We note that the resulting flat structure depends on the choice of the separatrices leaving each zero, on the homotopy class of the path $\rho$ as well as on the other given data. From (2) we see that for any saddle connection $\beta$, the length of $\beta$ on the new surface is at least $|\beta|-\varepsilon|\beta \cdot \rho| \geq|\beta|\left(1-\varepsilon^{1-2 r}\right)$ and since $|\beta| \geq \varepsilon^{2 r}$ on $S^{\prime}$, on the new surface $\beta$ must have length at least $\varepsilon^{r}$.

12.3. Admissible Constructions. We formalize the above construction as follows. Consider the following data:

- For $1 \leq i \leq p$ : surfaces $S_{i}^{\prime} \in \mathcal{H}\left(\alpha_{1}^{\prime}\right)$ with area $S_{i}^{\prime}<1$ and no saddle connections shorter than $2 \varepsilon^{r}$

- A vector $\gamma \in \mathbb{R}^{2}$, with $|\gamma|<\varepsilon$.

- For $1 \leq i \leq p$ : a pair of points $z_{i}, w_{i} \in S_{i}^{\prime}$ which are zeroes of orders $b_{i}^{\prime}, b_{i}^{\prime \prime}$ or marked points. The points may coincide. If they are distinct, we are also given a broken line $\rho_{i}$ joining $z_{i}$ and $w_{i}$. For any $\beta$ including those in a fixed homology basis,

$$
\left|\beta \cdot \rho_{i}\right| \leq|\beta| \varepsilon^{-2 r}
$$

where $\beta \cdot \rho_{i}$ is the algebraic intersection number.

- $\left|\rho_{i}\right| \leq C \varepsilon^{-r}$, where $C$ is a constant.

- If $w_{i}=z_{i}$ is a zero of order $a_{i}$, then a pair of numbers $a_{i}^{\prime}, a_{i}^{\prime \prime}$ with $a_{i}=a_{i}^{\prime}+a_{i}^{\prime \prime}$.

- A subset $J=\left\{i_{1}, \ldots, i_{q}\right\} \subset\{1, \ldots, p\}$, where $0 \leq q \leq p$. By convention, when $J=\emptyset$ we let $q=0$.

- For $1 \leq j \leq q$ : a number $h_{i_{j}}>0$ such that $\sum h_{i_{j}}|\gamma|+$ area $S_{i_{j}}<1$.

- For $1 \leq j \leq q$ : a number $t_{i_{j}}$ with $0 \leq t_{i_{j}}<|\gamma|$. 
Given the above data we construct a flat surface $S$ with homologous saddle connections of multiplicity $m$ with associated data $\left(J, a_{i}^{\prime}, a_{i}^{\prime \prime}, b_{k}^{\prime}, b_{k}^{\prime \prime}\right)$. Namely, for each $i$, if $z_{i}=w_{i}$ we use the figure eight construction and if $z_{i} \neq w_{i}$ we use the construction of creating a pair of holes giving two boundary curves $\gamma_{i}^{\prime}$ and $\gamma_{i}^{\prime \prime}$. For each $i$ either glue $\gamma_{i}^{\prime}$ directly to $\gamma_{i+1}^{\prime \prime}$ or, if $i=i_{j} \in\left\{i_{1}, \ldots, i_{q}\right\}=J$ glue in cylinders $C_{i_{j}}$ of "width" $|\gamma|$, "height" $h_{i_{j}}$ and "twist" $t_{i_{j}}$ to connect $\gamma_{i}^{\prime}$ to $\gamma_{i+1}^{\prime \prime}(i+1$ considered $\bmod p)$.

The resulting surface $S$ also depends on the data $h_{i_{j}}, t_{i_{j}}$ and $\rho_{i}$. Denote by $S^{\prime}$ the union of the pieces $S_{i}^{\prime}, h$ the vector of $h_{i_{j}}, t$ the vector of $t_{i_{j}}$ and $\rho$ the collection of $\rho_{i}$. Then we have an admissible construction

$$
\left(S^{\prime}, J, a_{i}^{\prime}, a_{i}^{\prime \prime}, b_{i}^{\prime}, b_{i}^{\prime \prime}, \rho, \gamma, h, t\right) \rightarrow S
$$

A relative homology basis for $S$ is given by:

- A relative homology basis of curves $\beta$ for each component $S_{i}^{\prime}$ of $S^{\prime}$.

- If one of the $z_{i}, w_{i}$ is a marked point, the curve $\rho_{i}$. If both are marked points, then in addition to $\rho$ a curve from some singularity on $S_{i}^{\prime}$ to $z_{i}$ (or to $w_{i}$ ) unless $S_{i}^{\prime}$ is a torus.

- A closed curve in the homology class of $\gamma_{i}$. The holonomy along this curve is $\gamma$.

- For each cylinder a curve joining its boundary singularities. The holonomy of this curve is $h+i t$.

We summarize the discussion as follows.

Lemma 12.2. Suppose that we

- either use at least one construction of creating a pair of holes; or

- if we use only figure eight constructions, we use at least one cylinder.

Then the resulting surface $S$ is a nondegenerate flat surface with no saddle connections other than the $\gamma_{i}$ shorter than $\varepsilon^{r}$ and thus it lies in $\mathcal{H}^{\varepsilon, \varepsilon^{r}}(\alpha, \mathcal{C})$.

The holonomy vectors $v_{S}(\beta)$ on $S$ of those curves $\beta$, which correspond to the original homology basis on $S_{i}^{\prime}$ are given by

$$
v_{S}(\beta)=v_{S^{\prime}}(\beta)+\left(\beta \cdot \rho_{i}\right) \gamma
$$

Example 12.3. In the example presented at Figure 11 we take two flat tori. In the picture they are represented by the large parallelograms with identified opposite sides $a_{1}$ to $a_{1}, b_{1}$ to $b_{1}$, and $a_{2}$ to $a_{2}, b_{2}$ to $b_{2}$. In fact, the zeroes $z_{1}, w_{1}$, and $z_{2}, w_{2}$ are fake here: all these points are just marked points of the tori. In this example we choose the vector $\vec{\gamma}$ to be vertical.

We cut a rectangular domain out of the interior of each of the two tori in such way that the vertical side of each of the two removed rectangles is equal to $\vec{\gamma}$. Then we identify the horizontal sides $d_{1}$ to $d_{1}$ and $d_{2}$ to $d_{2}$. We get a pair of tori, each provided with a pair of holes.

Finally, we join the hole $\overline{w_{1} w_{1}^{\prime}}$ on the first torus to the hole $\overline{z_{2} z_{2}^{\prime}}$ on the second torus by a cylinder (in the picture the cylinder is represented by a narrow stripe with identified opposite sides $c_{1}$ to $c_{1}$ ); we also join the hole $\overline{w_{2} w_{2}^{\prime}}$ on the second torus to the hole $\overline{z_{1} z_{1}^{\prime}}$ on the first torus by a cylinder (in the picture this second cylinder is represented by a narrow stripe with identified opposite sides $c_{2}$ to $c_{2}$ ). 


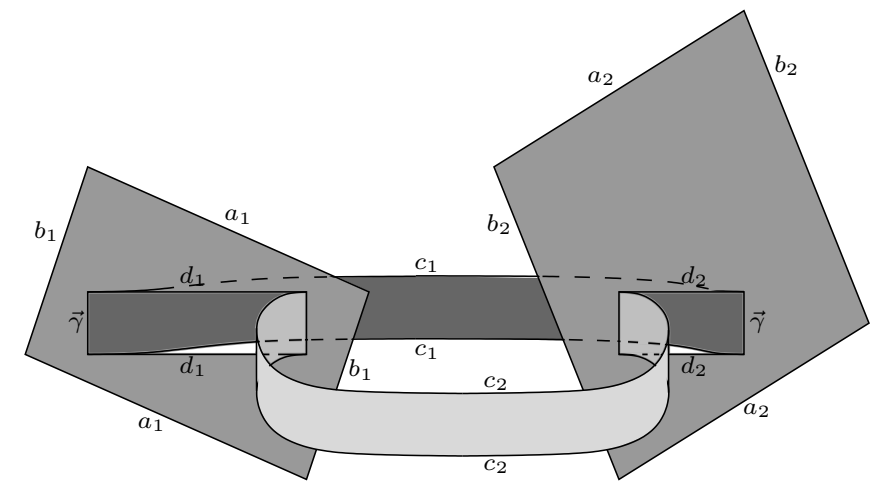

FIGURE 11. In this example we apply the construction of creating a pair of holes simultaneously to two tori (represented by large parallelograms with identified opposite sides) and then glue in a pair of cylinders (represented by the narrow stripes with identified opposite sides).

We get a flat surface of genus $g=3$ with four simple zeroes. By construction the surface contains two cylinders filled with closed regular homologous geodesics with holonomy $\vec{\gamma}$ (these cylinders are represented by narrow stripes with horizontal sides $c_{1}$ and $c_{2}$ ). In other words the saddle connection represented by $\gamma$ has multiplicity 2 . Note that the two cylinders would survive under any sufficiently small deformation of the surface, which means that this configuration is generic.

Now we return to the discussion at the beginning of part II. We are given a set of homologous saddle connections $\gamma_{i}$ and a surface $S \in \mathcal{H}^{\varepsilon, 3 \varepsilon}(\alpha, \mathcal{C})$. We may cut $S$ along the $\gamma_{i}$ to form a collection of surfaces $S_{i}$, each with a pair of boundary components. We may then choose a path $\tau_{i}$ in each $S_{i}$ joining the cone points and shrink the boundary circles giving surfaces $S_{i}^{\prime}$ in $\mathcal{H}\left(\alpha_{i}^{\prime}\right)$. An analysis similar to that given in the section on creating a pair of holes shows that we may choose the $\tau_{i}$ so that the resulting surfaces lie in $\mathcal{F}^{\prime}$.

Our main objective is now to show that the degeneration of a surface to a point on the principal boundary and the admissible construction of a surface from a point on the principal boundary are essentially inverse operations. This will allow us to compute the constants for the counting problem. The complicating issue is that the degeneration depends on the paths $\tau_{i}$, while the admissible construction depends on the choice of $\rho_{i}$ and these curves might be not the same. In particular, since lengths of curves may change by $\varepsilon$ during the construction, the shortest curve may change. However, changing from one path to another amounts to a change of basis and can be described.

Recall that we have fixed a homology basis $\beta_{i}$ valid for all $S^{\prime} \in \mathcal{F}$. The holonomy along the basis allows one to define a metric $d(\cdot, \cdot)$ on $\mathcal{F}^{\prime}$. The length of the $\beta_{i}$ satisfy $\left|\beta_{i}\right| \leq C \varepsilon^{-r}$ for a fixed constant $C$. Fix a pair of zeroes of a given $S_{i}^{\prime}$ and a path $\rho_{i}$ joining them which does not pass within $\varepsilon+\varepsilon^{2 r}$ of a singularity or of itself. We may find a ball of radius $\varepsilon^{2 r}$ about $S_{i}$ in the metric $d$ such that for all other surfaces in the ball we may perform the admissible construction using the same paths $\rho_{i}$ since for any surface in the ball the corresponding paths do not come within $\varepsilon$ of 
a singularity or themselves. Thus we can find a finite collection of balls $B\left(x_{j}, r_{j}\right)$ centered at such points $x_{j}$ of radius $\varepsilon^{2 r}$ that cover $\mathcal{F}^{\prime}$ such that for each $B\left(x_{j}, r_{j}\right)$, for each component $S_{i}^{\prime}$ of $S^{\prime}$ for which $z_{i} \neq w_{i}$ there is a fixed curve $\rho_{i, j}$ joining $z_{i}$ to $w_{i}$ and so that we may form the admissible construction for each $x \in x_{j}$.

We may use the cover to define a partition of $\mathcal{F}^{\prime}$ by a finite collection of sets $U_{j} \subset B\left(x_{j}, r_{j}\right)$. Now we can give a well defined assignment

$$
\sigma: \mathcal{F}^{\prime} \rightarrow \mathcal{H}_{1}^{\varepsilon, \varepsilon^{r}}(\alpha, \mathcal{C})
$$

Namely each $S^{\prime}$ belongs to a unique $U_{j}$. Then use $\rho_{i, j}$ to assign to $S^{\prime}$ a closed surface $S=\sigma\left(S^{\prime}\right)$ by the admissible construction.

As in the previous section, we have the possibility of a construction for $-\gamma$ coinciding with a construction with $\gamma$ and also the possibility of stratum interchange. However, even without considering these symmetries, it is possible because of the change of transversals from one set $U_{j}$ to another, that the construction is not $1-1$. We prove

Proposition 12.4. There is $E^{\prime} \subset \mathcal{F}^{\prime}$ such that

- $\operatorname{Vol}\left(\mathcal{F}^{\prime}-E^{\prime}\right)=O\left(\varepsilon^{1-3 r}\right)$,

- On a subset of $E^{\prime}$ of full volume, $\sigma$ is $1-1$ except for the $\gamma \rightarrow-\gamma$ and stratum interchange symmetries.

- $\operatorname{Vol}\left(\mathcal{H}_{1}^{\varepsilon, \varepsilon^{r}}(\alpha, \mathcal{C})-\sigma\left(E^{\prime}\right)\right)=o\left(\varepsilon^{2}\right)$

Proof. Define $E^{\prime}$ to be the set of points which are at least $C \varepsilon^{1-3 r}$, from the boundary of any ball. This means that if $S^{\prime} \in E^{\prime} \cap U_{j}$ then the $C \varepsilon^{1-3 r}$ neighborhood of $S^{\prime}$ is contained in $U_{j}$. It also implies

$$
\operatorname{Vol}\left(\mathcal{F}^{\prime}-E^{\prime}\right)=O\left(\varepsilon^{1-3 r}\right)
$$

We prove $\sigma$ is $1-1$ on almost all of $E^{\prime}$ except for possible stratum interchange and $\gamma \rightarrow-\gamma$ symmetries. If $S_{1}^{\prime} \neq S_{2}^{\prime}$ belong to the same $U_{j}$ then the collection of surfaces $\sigma\left(S_{1}^{\prime}\right)$ and $\sigma\left(S_{2}^{\prime}\right)$ are constructed with the same broken curve and yet differ on holonomy for a set of basis curves. The surfaces can be isomorphic only if the isomorphism does not preserve the basis. If the set of configuration curves are preserved then we have the stratum interchange or $\gamma \rightarrow-\gamma$ symmetry. If they are not preserved, then $S^{\prime}$ contains a curve of length at most $\varepsilon$ and this possibility has been ruled out by the definition of $\mathcal{F}^{\prime}$. The remaining possibility is if for a fixed $S^{\prime}$ two or more of the remaining surfaces in $\sigma\left(S^{\prime}\right)$ are isomorphic by an isomorphism that preserves the set of curves in the configuration. But such an isomorphism must arise from an automorphism of $S^{\prime}$ and such $S^{\prime}$ have volume 0 .

If $S_{1}^{\prime}, S_{2}^{\prime} \in E^{\prime}$ belong to different $U_{j}$, then their distance apart in the metric $d$ is at least $2 C \varepsilon^{1-3 r}$ so their holonomy on some basis curve $\beta_{i}$ differs by at least $2 C \varepsilon^{1-3 r}$. However, by (28) the change in holonomy in passing to $\sigma\left(S^{\prime}\right)$ from $S^{\prime}$ and $\sigma\left(S^{\prime \prime}\right)$ from $S^{\prime \prime}$ is at most $\varepsilon\left|\beta_{i} \cdot \rho_{i, j}\right| \leq \varepsilon\left|\beta_{i}\right| \varepsilon^{-2 r} \leq C \varepsilon^{1-3 r}$ and so again $\sigma\left(S_{1}^{\prime}\right)$ and $\sigma\left(S_{2}^{\prime}\right)$ again differ on $\beta_{i}$ and we have the same conclusion as before.

We now show the third statement. Given the transversals $\tau_{i}$, let $E$ be the subset of $\mathcal{H}_{1}^{\varepsilon, 3 \varepsilon^{r}}(\alpha, \mathcal{C})$ consisting of those $S$ whose degeneration yields surfaces $S^{\prime}$ which are at least $3 C \varepsilon^{1-3 r}$ from the boundary of any ball. We have

$$
\operatorname{Vol}\left(\mathcal{H}_{1}^{\varepsilon, 3 \varepsilon^{r}}(\alpha, \mathcal{C})-E\right)=o\left(\varepsilon^{2}\right)
$$

For some $j$, the resulting surface $S^{\prime} \in U_{j} \cap B\left(x_{j}, r_{j}-3 \varepsilon^{1-3 r}\right)$. However, since it may not be the case that $\tau_{i}=\rho_{i, j}$, it may not be true that $\sigma\left(S^{\prime}\right)=S$. Now we 
claim that there is some $S^{\prime \prime} \in E^{\prime}$ such that $S=\sigma\left(S^{\prime \prime}\right)$. To prove the claim, notice that since $B\left(x_{j}, r_{j}\right)$ is a ball and since $\left|\beta \cdot \tau_{i}\right| \leq C \varepsilon^{-3 r},\left|\beta \cdot \rho_{i, j}\right| \leq C \varepsilon^{-3 r}$, and $|\gamma| \leq \varepsilon$, the point in $\mathbb{R}^{n^{\prime}}$ defined by the collection of vectors

$$
v_{S^{\prime}}(\beta)+\left(\sum_{i}\left(\beta \cdot \tau_{i}\right)-\left(\beta \cdot \rho_{i, j}\right)\right) \gamma
$$

as $\beta$ runs over the homology basis, corresponds to the holonomy for a unique $S^{\prime \prime} \in B\left(x_{j}, r_{j}-C \varepsilon^{1-3 r}\right)$. so that $S^{\prime \prime} \in E^{\prime}$. But now by (28), $\sigma\left(S^{\prime \prime}\right)$ has the same holonomy as $S$ along the basis and so is isomorphic to it, proving the claim. The third statement now follows from the estimate $\operatorname{Vol}\left(\mathcal{H}_{1}^{\varepsilon, \varepsilon^{r}}(\alpha, \mathcal{C})-\mathcal{H}_{1}^{\varepsilon, 3 \varepsilon^{r}}(\alpha, \mathcal{C})\right)=$ $o\left(\varepsilon^{2}\right)$, which follows from Lemma 7.1 .

\section{Computing the Siegel-Veech Constants for Connected Strata}

Recall that $q$ is the number of cylinders we shall attach. Let $n_{i}=\operatorname{dim}_{\mathbb{R}} \mathcal{H}\left(\alpha_{i}\right)$. We also define $d_{i}$ to be equal to $n_{i}$ except in the following situations. If $S_{i}$ has a single marked point which is not a zero and $S_{i}$ is not the torus, we have $d_{i}=n_{i}+2$. If there is a pair of marked points, and the surface is not the torus $d_{i}=n_{i}+4$. If the surface is a torus then $d_{i}=n_{i}+2$. Then,

$$
\operatorname{dim}_{\mathbb{R}} \mathcal{H}(\alpha)=2 q+2+\sum_{i=1}^{p} d_{i},
$$

where $\alpha$ is the stratum such that $S \in \mathcal{H}(\alpha)$.

13.1. Computation of the Constants. Recall that $d \nu(S)$ is the measure in $\mathcal{H}(\alpha)$ and $d \nu\left(S_{i}^{\prime}\right)$ is the measure in $\mathcal{H}\left(\alpha_{i}\right)$. As in Part I we will obscure the distinction between $\gamma$ a saddle connection and its holonomy so that we will use $\gamma$ to refer to a vector as a variable of integration in a disc of radius $\varepsilon$. We let $M$ be the number which computes of fixed data $\left(S^{\prime}, J, a_{i}^{\prime}, a_{i}^{\prime \prime}, b_{k}^{\prime}, b_{k}^{\prime \prime}, \gamma, h, t\right)$ the number of $S=\sigma(S)$ that can be built. This number will be computed in the next sections. We first assume that $\mathcal{H}(\alpha)$ is connected. If we choose a homology basis $\beta_{i}$ for the $S_{i}^{\prime}$, and let $h o l_{S}\left(\beta_{i}\right)$ be the holonomy of $\beta_{i}$ on the surface $S$, then the measure $d \nu$ restricted to the image $\sigma\left(E^{\prime}\right)$ under the admissible construction is the product measure of $M d \gamma d t_{i} d h_{i}$ and $\prod d\left(h o l_{S}\left(\beta_{i}\right)\right)$. By (28)

$$
\prod d\left(h o l_{S}\left(\beta_{i}\right)\right) d \gamma=d \nu^{\prime} d \gamma
$$

and again since $|\gamma| \leq \varepsilon$ we can write

$$
d \nu(S)=d \gamma \prod_{i=1}^{p} d \nu^{\prime}\left(S_{i}^{\prime}\right) \prod_{i=1}^{q} d h_{i} d t_{i}
$$

except for the following situations. If $z_{i}$ is a marked point and $w_{i}$ is a zero then we have the additional factor $d z\left(S_{i}\right)$ in the term $d \nu_{i}$. If $z_{i}$ is a zero and $w_{i}$ is a marked point we have $d w\left(S_{i}\right)$. If $w_{i}=z_{i}$ is a marked point, we have $d z\left(S_{i}\right)$ and if $z_{i} \neq w_{i}$ are both marked points we have the factor $d z\left(S_{i}\right) d w\left(S_{i}\right)$.

Note that we could also consider strata with one or two marked points. The stratum would be a fiber space over the stratum $\mathcal{H}_{1}(\alpha)$. The fiber over a point would be the flat surface if there is one marked point or product of flat surfaces if there are two, the flat surface representing that point in $\mathcal{H}_{1}(\alpha)$. The measure on the fiber space is then locally the product measure of the base with the area (or product of area measures) on the fiber. 
Now for each $S_{i}$ we either perform the figure eight or creating a pair of holes construction. In the latter case we remove or add a parallelogram $P_{i}$. Thus area $S=\sum_{i}\left(\right.$ area $\left.S_{i}\right)+h_{i}|\gamma| \pm$ area $P_{i}$, (where area $P_{i}=0$ if we perform the figure eight construction). If $S \in C\left(\mathcal{H}_{1}^{\varepsilon, 3 \varepsilon^{r}}(\alpha, \mathcal{C})\right)$ then the normalization says that $|\gamma| \leq \varepsilon \sqrt{(\operatorname{area} S)}$. Hence,

$$
|\gamma|^{2} \leq \varepsilon^{2}\left(\sum_{i}\left(\operatorname{area} S_{i}+h_{i}|\gamma| \pm \operatorname{area} P_{i}\right)\right)
$$

i.e.

$$
\sum_{i} h_{i} \geq \frac{|\gamma|}{\varepsilon^{2}}-\frac{\sum_{i}\left(\operatorname{area} S_{i} \pm \operatorname{area} P_{i}\right)}{|\gamma|}
$$

The expression on the right is positive when $|\gamma|>\varepsilon \sqrt{\left(\sum_{i} \text { area } S_{i} \pm \text { area } P_{i}\right)}$.

Let

$$
W=\prod_{i} \operatorname{Vol}\left(\mathcal{H}_{1}\left(\alpha_{i}\right)\right)
$$

For $s>0$, let $D(s)=\left\{\left(r_{1}, \ldots, r_{p}\right) \quad: \quad \sum_{i} r_{i}^{2}<s\right\}$. Then using the fact that area $P_{i}=O\left(\varepsilon^{1-r}\right)$, Proposition 12.4 the fact that $\operatorname{Vol}\left(\prod_{i} \mathcal{H}_{1}\left(\alpha_{i}\right)-\mathcal{F}^{\prime}\right)=O\left(\varepsilon^{2 r}\right)$ and $|\gamma| \leq \varepsilon$, we have

$$
\begin{aligned}
& \nu\left(C\left(\mathcal{H}_{1}^{\varepsilon, \varepsilon^{r}}(\alpha)\right)\right)=W M .
\end{aligned}
$$

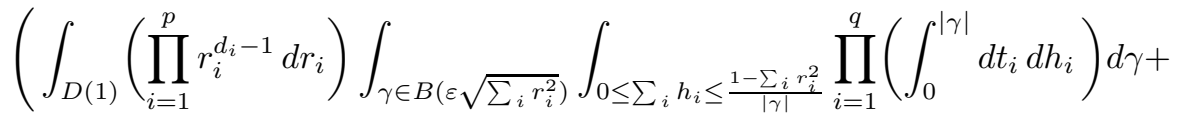

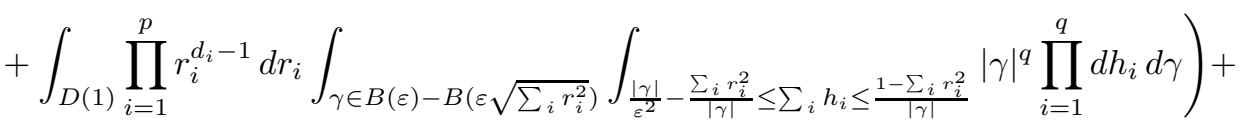

$$
\begin{aligned}
& +o\left(\varepsilon^{2}\right)= \\
& =W M \cdot\left(\int_{D(1)} \prod_{i=1}^{p} r_{i}^{d_{i}-1} d r_{i} \int_{\gamma \in B\left(\varepsilon \sqrt{\sum_{i} r_{i}^{2}}\right)} \frac{1}{q !}\left(1-\sum_{i} r_{i}^{2}\right)^{q} d \gamma+\right. \\
& \left.+\int_{D(1)} \prod_{i=1}^{p} r_{i}^{d_{i}-1} d r_{i} \int_{\gamma \in B(\varepsilon)-B\left(\varepsilon \sqrt{\sum_{i} r_{i}^{2}}\right)} \frac{1}{q !}\left[\left(1-\sum_{i} r_{i}^{2}\right)^{q}-\left(\frac{|\gamma|^{2}}{\varepsilon^{2}}-\sum_{i} r_{i}^{2}\right)^{q}\right] d \gamma\right)+ \\
& +o\left(\varepsilon^{2}\right)
\end{aligned}
$$

Performing the integral over $\gamma$ we obtain 


$$
\begin{aligned}
& \nu\left(C\left(\mathcal{H}_{1}^{\varepsilon, \varepsilon^{r}}(\alpha, \mathcal{C})\right)\right)= \\
&=\frac{\pi \varepsilon^{2} W M}{q !} \int_{D(1)}\left(\prod_{i=1}^{p} r_{i}^{d_{i}-1}\right)\left(1-\sum_{i} r_{i}^{2}\right)^{q}\left(\sum_{i} r_{i}^{2}\right) \prod_{i} d r_{i} \\
&+\frac{\pi \varepsilon^{2} W M}{q !} \int_{D(1)}\left(\prod_{i=1}^{p} r_{i}^{d_{i}-1}\right)\left(1-\sum_{i} r_{i}^{2}\right)^{q}\left(1-\sum_{i} r_{i}^{2}\right) \prod_{i} d r_{i} \\
& \quad-\frac{\pi W M}{q !} \int_{D(1)}\left(\prod_{i=1}^{p} r_{i}^{d_{i}-1}\right) \int_{\varepsilon \sqrt{\sum_{i} r_{i}^{2}}}^{\varepsilon}\left(\frac{s^{2}}{\varepsilon^{2}}-\sum_{i} r_{i}^{2}\right)^{q} 2 s d s \prod_{i} d r_{i}+o\left(\varepsilon^{2}\right) \\
&=-\frac{\pi \varepsilon^{2} W M}{q !} \int_{D(1)}\left(\prod_{i=1}^{p} r_{i}^{d_{i}-1}\right)\left(1-\sum_{i} r_{i}^{2}\right)^{q} \prod_{i} d r_{i} \\
&-\frac{\pi \varepsilon^{2} W M}{(q+1) !} \int_{D(1)}\left(\prod_{i=1}^{p} r_{i}^{d_{i}-1}\right)\left(1-\sum_{i} r_{i}^{2}\right)^{q+1} \prod_{i} d r_{i}+o\left(\varepsilon^{2}\right)
\end{aligned}
$$

where we have used the change of variable $u=\left(s^{2} / \varepsilon^{2}-\sum r_{i}^{2}\right)$ in the last line. We now make the change of variable $x_{i}=r_{i}^{2}$. Let $b_{i}=d_{i} / 2-1$, so that $r_{i}^{d_{i}-1} d r_{i}=$ $(1 / 2) x_{i}^{b_{i}} d x_{i}$. Then,

$$
\begin{aligned}
\nu\left(C\left(\mathcal{H}_{1}^{\varepsilon, \varepsilon^{r}}(\alpha, \mathcal{C})\right)\right)=\frac{\pi \varepsilon^{2} W M}{2^{p}} & \int_{\sum_{i} x_{i} \leq 1} x_{1}^{b_{1}} \ldots x_{p}^{b_{p}}\left[\frac{1}{q !}\left(1-\sum_{i} x_{i}\right)^{q}\right. \\
& \left.-\frac{1}{(q+1) !}\left(1-\sum_{i} x_{i}\right)^{q+1}\right] d x_{1} \ldots d x_{p}+o\left(\varepsilon^{2}\right)
\end{aligned}
$$

Evaluating the integral from the inside out via repeated application of the identity

$$
\int_{0}^{u} x^{a}(u-x)^{b} d x=\frac{a ! b !}{(a+b+1) !} u^{a+b+1}
$$

yields

$$
\begin{aligned}
& \nu\left(C\left(\mathcal{H}_{1}^{\varepsilon, \varepsilon^{r}}(\alpha, \mathcal{C})\right)\right)= \\
& \quad=\frac{\pi \varepsilon^{2} W M}{2^{p}}\left[\frac{b_{1} ! \ldots b_{p} !}{\left(b_{1}+\cdots+b_{p}+p+q\right) !}-\frac{b_{1} ! \ldots b_{p} !}{\left(b_{1}+\cdots+b_{p}+p+q+1\right) !}\right]+o\left(\varepsilon^{2}\right)= \\
& \quad=\frac{\pi \varepsilon^{2} W M}{2^{p}} \frac{b_{1} ! \ldots b_{p} !}{\left(b_{1}+\cdots+b_{p}+p+q-1\right) !} \cdot \frac{1}{b_{1}+\cdots+b_{p}+p+q+1}+o\left(\varepsilon^{2}\right)
\end{aligned}
$$

Since $b_{1}+\cdots+b_{p}+p+q+1=n / 2$, we have

$$
\nu\left(C\left(\mathcal{H}_{1}^{\varepsilon, \varepsilon^{r}}(\alpha, \mathcal{C})\right)\right)=\frac{\pi \varepsilon^{2} W M}{2^{p-1}} \frac{b_{1} ! \ldots b_{p} !}{n\left(\frac{n}{2}-2\right) !}+o\left(\varepsilon^{2}\right)
$$


Now taking the limit as $\varepsilon \rightarrow 0$, we get

$$
\begin{aligned}
& c=\lim _{\varepsilon \rightarrow 0} \frac{\nu_{1}\left(C\left(\mathcal{H}_{1}^{\varepsilon, \varepsilon^{r}}(\alpha, \mathcal{C})\right)\right)}{\pi \varepsilon^{2} \nu_{1}\left(\mathcal{H}_{1}(\alpha)\right)}= \\
&=M \cdot \frac{1}{2^{p-1}} \cdot \frac{\prod_{i=1}^{p}\left(\frac{d_{i}}{2}-1\right) !}{\left(\frac{n}{2}-2\right) !} \cdot \frac{\prod_{i=1}^{p} \operatorname{Vol}\left(\mathcal{H}_{1}\left(\alpha_{i}^{\prime}\right)\right)}{\operatorname{Vol}\left(\mathcal{H}_{1}(\alpha)\right)}
\end{aligned}
$$

In order to compute the combinatorial constant $M$ let us study in more detail how, given the admissible construction $\left(S^{\prime}, J, a_{i}^{\prime}, a_{i}^{\prime \prime}, b_{k}^{\prime}, b_{k}^{\prime \prime}, \rho, \gamma, h, t\right) \rightarrow S$, the resulting flat surface $S$ is made up of subsurfaces. The resulting surface $S$ has two groups of zeroes: the ones inherited from the components $S_{i}^{\prime}$ without any changes, and the newborn ones. Every newborn zero has at least one curve $\gamma_{i}$ passing through it; the curves $\gamma_{i}$ do not pass through the zeroes of the first group. Actually, it is easy to see from our construction, that the collection of curves $\gamma_{i_{j}}$ passing through a zero of $S$ has the form $\gamma_{i}, \gamma_{i+1}, \ldots, \gamma_{i+s}$, with consecutive indices (where as usual $p+1$ is identified with 1 , so $p$ is followed by 1 ). It is easy to give a description of the nature of the corresponding set $\{i, i+1, \ldots, i+s\}$.

Any newborn zero is formed by one of the following three constructions. The reader may refer to figure 10 .

- Type I. There is a chain of consecutive surfaces $S_{j_{r}+1} \rightarrow S_{j_{r}+2} \rightarrow \cdots \rightarrow S_{j_{r}+s}$ glued directly. To each surface of the chain we apply the figure eight construction. The first surface of the chain $S_{j_{r}+1}$ is glued to the preceding surface $S_{j_{r}}$ by a cylinder and similarly for the last surface of the chain $S_{j_{r}+s}$ and its successor. In our notation we have $j_{r}+s=j_{r+1}$, where both $j_{r}, j_{r+1} \in J$, and $s \geq 0$.

In this case the newborn zero has order

$$
\sum_{k=1}^{s}\left(a_{j_{r}+k}+2\right)
$$

where $a_{j_{r}+k}=a_{j_{r}+k}^{\prime}+a_{j_{r}+k}^{\prime \prime}$. By convention $s=0$ stands for the case when multiplicity $p$ is equal to $1, J=\{1\}$, and we have a single surface $S_{1}$ to which we apply figure eight construction and glue in a cylinder.

The left drawing in figure 10 illustrates the situation when $s=2$. The figure eight construction is applied to each of the two surfaces and they are glued to each other directly. Each is glued to another surface by a cylinder.

- Type II. Now we have the following chain of surfaces $S_{j_{r}+1}, S_{j_{r}+2}, \ldots, S_{j_{r}+s+1}$ glued directly. We apply the figure eight construction to all surfaces except the last, to which we apply the construction of creating a pair of holes. The first surface $S_{j_{r}+1}$ of the chain is glued to $S_{j_{r}}$ by a cylinder. The value $s=0$ is allowed: in this case we have a single surface $S_{j_{r}+1}$ to which we apply a construction of creating a pair of holes, and then attach a cylinder to the zero $z_{j_{r}+1}$ of order $b_{j_{r}+1}^{\prime}+1$. In this case the newborn zero has order

$$
\sum_{k=1}^{s}\left(a_{j_{r}+k}+2\right)+\left(b_{j_{r}+s+1}^{\prime \prime}+1\right)
$$

We may have the symmetric picture, when the surface $S_{j}$ to which we apply the construction of creating a pair of holes is at the beginning of the chain, then we glue directly the surfaces $S_{j+1}, \ldots, S_{j+s}$ to which we apply figure eight construction, and to the last surface $S_{j+s}$ (where $j+s \in J$ equals some $j_{r}$ ) we glue a cylinder. We 

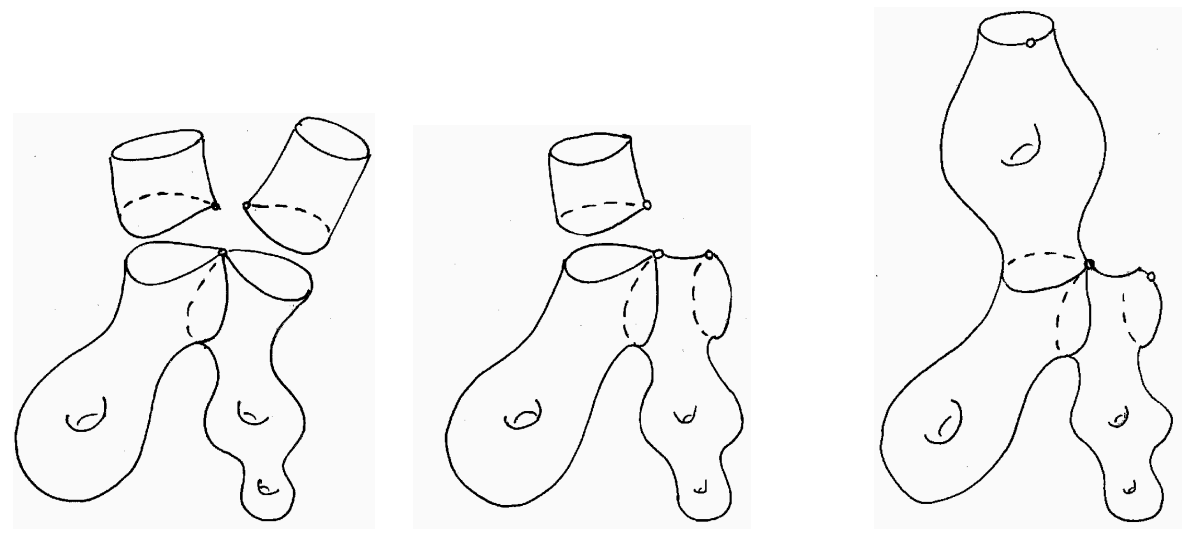

FiguRE 12. Newborn zeroes of three different types.

have a symmetric formula for the order of the zero:

$$
\left(b_{j}^{\prime}+1\right)+\sum_{k=1}^{s}\left(a_{j+k}+2\right)
$$

The middle drawing in Figure 10 illustrates this with $s=2$. A figure eight construction is applied to the genus 1 surface and a construction of creating a pair of holes to the genus 2 surface. The first surface is glued to a cylinder.

- Type III. Finally, we may have surfaces $S_{k}, \ldots, S_{k+s+1}$ to which we apply the construction of creating a pair of holes boarding both ends of the chain; we apply the figure eight construction to all interior surfaces $S_{k+1} \ldots S_{k+s}$; we glue all surfaces directly. The value $s=0$ is admissible: by convention it means that we have only two surfaces $S_{k}$ and $S_{k+1}$ in the chain to which we apply the construction of creating a pair of holes and then glue directly $\gamma_{k}^{\prime \prime}$ to $\gamma_{k+1}^{\prime}$. In this case we get the newborn zero of the following order

$$
\left(b_{k}^{\prime}+1\right)+\sum_{i=1}^{s}\left(a_{k+i}+2\right)+\left(b_{k+s+1}^{\prime \prime}+1\right)
$$

The last drawing in figure 10 illustrates this situation with $s=2$. A construction of creating a pair of holes is applied to one torus and the surface of genus 2 . The figure eight construction is applied to the middle torus. One boundary component of it is glued to a boundary component of the genus 2 surface; the other boundary component glued to a boundary component of the other torus.

We now adopt the following notation to describe how the surfaces are put together.

Notation The symbol $\rightarrow$ corresponds to direct gluing of the surfaces; the symbol $\Rightarrow$ corresponds to a cylinder joining two consecutive surfaces. If a number is written as a sum of two numbers $a_{i}^{\prime}+a_{i}^{\prime \prime}$ with a bar over the sum, we apply the figure eight construction to a zero of order $a_{i}=a_{i}^{\prime}+a_{i}^{\prime \prime}$ breaking it up into zeroes of order $a_{i}^{\prime}$ and $a_{i}^{\prime \prime}$; if there are two multiplicity numbers, $\bar{b}_{i}^{\prime}, \bar{b}_{i}^{\prime \prime}$, we apply the construction of creating a pair of holes. By convention $b_{i}^{\prime}$ is the left one, $b_{i}^{\prime \prime}$ is the right one. Say, in $(4, \overline{3}, 2, \overline{1})$ we have $b_{1}^{\prime}=3, b_{1}^{\prime \prime}=1$, and in $(\overline{0+0})$ we have $a_{2}=a_{2}^{\prime}=a_{2}^{\prime \prime}=0$. 
Example 13.1. Consider the following collection of flat surfaces organized in a cyclic order

$\rightarrow(4, \overline{3}, 2, \overline{1}) \rightarrow(\overline{0+0}) \rightarrow(\overline{4+2}, 2) \rightarrow(\overline{9}, 8, \overline{7}) \rightarrow(\overline{5}, \overline{3}) \Rightarrow(\overline{2+2}, 2) \rightarrow(5, \overline{1+2}) \Rightarrow(\overline{1+0}, 1) \rightarrow(2, \overline{0+0}) \rightarrow$

$\rightarrow S_{1} \rightarrow S_{2} \rightarrow S_{3} \rightarrow S_{4} \rightarrow S_{5} \Rightarrow S_{6} \rightarrow S_{7} \Rightarrow S_{8} \rightarrow S_{9} \rightarrow$

In the example above we glue $S_{5}$ to $S_{6}$ with a cylinder, and $S_{7}$ to $S_{8}$ with a cylinder; all the other gluings are direct. Thus in this example we have $p=9$, and $J=\{5,7\}$. The chain $S_{1} \rightarrow S_{2} \rightarrow S_{3} \rightarrow S_{4}$ gives a newborn zero of type III. It has order $(1+1)+((0+2)+(6+2))+(9+1)=22$; there are 3 separatrix loops $\gamma_{1}, \gamma_{2}, \gamma_{3}$ passing through this newborn zero. The loops $\gamma_{1}$ and $\gamma_{2}$ bound the surface $S_{2}$ and bound angles $2 \pi$ with each other. The loops $\gamma_{2}, \gamma_{3}$ bound $S_{3}$ with angles $10 \pi$ and $6 \pi$. The next chain $S_{4} \rightarrow S_{5}$ gives a newborn zero of type III; it has order $(7+1)+(5+1)=14$; there is only one separatrix loop $\gamma_{4}$ passing through this zero. The next chain is $S_{5} \Rightarrow$. It is of type II; it gives a newborn zero of order $3+1=4$ with a separatrix loop $\gamma_{5}$ passing through it. The next chain $\Rightarrow S_{6} \rightarrow S_{7} \Rightarrow$ gives a newborn zero of order $(4+2)+(3+2)=11$ of type I, and three separatrix loops $\gamma_{6}, \gamma_{7}, \gamma_{8}$ passing through it. The surface $S_{6}$ is bounded by $\gamma_{6}$ and $\gamma_{7}$ which make angles $8 \pi$ and $4 \pi$ at the zero of order 11 , while $S_{7}$ is bounded by $\gamma_{7}$ and $\gamma_{8}$ which make angles $6 \pi$ and $4 \pi$ at the zero. Finally, the last chain $\Rightarrow S_{8} \rightarrow S_{9} \rightarrow S_{1}$ produces a newborn zero of type II. Its order is $(1+2)+(0+2)+(3+1)=9$; there are three separatrix loops $\gamma_{9}, \gamma_{10}, \gamma_{11}$ passing through it. The loops $\gamma_{9}$ and $\gamma_{10}$ bound the surface $S_{8}$ making angles $4 \pi$ and $2 \pi$ with each other while $\gamma_{10}$ and $\gamma_{11}$ bound $S_{9}$ making angles $2 \pi$ and $2 \pi$ with each other.

The resulting surface inherits the zeroes of orders $4,2,2,8,2,5,1,2$ which are coming unchanged from the components $S_{i}$ (the integers which are not barred.) It also has the newborn zeroes of orders $22,14,4,11,9$. Thus the resulting surface $S$ belongs to the stratum $\mathcal{H}(22,14,11,9,8,5,4,4,2,2,2,2,1)$.

13.2. Stratum Interchange and $\gamma \rightarrow-\gamma$ Symmetry. In this section we discuss the possible symmetries of the admissible construction

$$
\left(S, a_{i}^{\prime}, a_{i}^{\prime \prime}, b_{k}^{\prime}, b_{k}^{\prime \prime}, \rho, \gamma, h, t\right) \rightarrow S
$$

In many aspects these symmetries are analogous to the symmetries discussed in Section 9.4 We again consider two settings: the first one with more restrictions, the second one with fewer restrictions.

Problem 1. We assume that all zeroes $z_{1}, \ldots, z_{l}$ of the surface $S$ are numbered. We fix the zeroes $z_{1}, \ldots, z_{r} \in S, z_{i} \neq z_{j}$, for $i \neq j$, of orders $m_{1}, \ldots, m_{r}$ correspondingly. We assume that the flat surface $S$ possesses exactly $s$ loops of saddle connections $\gamma_{1}, \ldots, \gamma_{s}$ homologous to some fixed loop $\gamma$. We assume that every loop $\gamma_{i}$ starts and ends at one of the zeroes $z_{1}, \ldots, z_{r}$.

Cutting along the loops $\gamma_{i}, i=1, \ldots, r$ we perform the surgery as described above decomposing $S$ into the collection $S_{i}^{\prime}$. The collection is organized in a cyclic order as described above.

We fix the types $\alpha_{i}^{\prime}$ of the surfaces $S_{i}^{\prime}$, the distinguished zeroes (marked points) $w_{i}, z_{i} \in S_{i}^{\prime}$, and the numbers $\left(a_{i}^{\prime}, a_{i}^{\prime \prime}\right),\left(b_{k}^{\prime}, b_{k}^{\prime \prime}\right)$ where both types of pairs are ordered. We fix, whether $S_{j}^{\prime}$ is joined to $S_{j}^{\prime}$ by a cylinder, or directly.

We start with the case when we fix also the following data. We assume that $z_{1}, \ldots, z_{r}$ are the newborn zeroes numbered with respect to this cyclic order. We 
assume that the zeroes $z_{r+1}, \ldots, z_{l_{1}}$ lie in $S_{1}$, the zeroes $z_{l_{1}+1}, \ldots, z_{l_{1}+l_{2}}$ lie in $S_{2}$, $\ldots$, the zeroes $z_{l_{1}+\cdots+l_{p-1}+1}, \ldots, z_{l_{1}+\cdots+l_{p}}$ lie in $S_{p}$. Here $l=l_{1}+\cdots+l_{p}=\operatorname{card}(\alpha)$.

Problem 2. Now consider the problem with fewer constraints. We count the number of occurrences of configurations of loops of saddle connections described in the previous problem with the only difference that now we assume all the zeroes to be "anonymous". In other words, we keep all geometric information on configuration (number $r$ of zeroes involved; number $s$ of loops of homologous saddle connections; types $\alpha_{i}^{\prime}$ of the surfaces $S_{i}^{\prime}$, and the cyclic order of their appearances; numbers $\left(a_{i}^{\prime}, a_{i}^{\prime \prime}\right),\left(b_{j}^{\prime}, b_{j}^{\prime \prime}\right)$, where both types of pairs are ordered; the way (by means of a cylinder or directly) $S_{i}^{\prime}$ is joined to $S_{i+1}^{\prime}$ ).

However, if we have two zeroes $P_{i}, P_{j}$ of the same degree on the surface $S$, and, neither of them is "newborn", in the setting of Problem 2 we do not distinguish cases, when $P_{i}$ lies in $S_{q}^{\prime}$ and $P_{j}$ gets to $S_{t}^{\prime}$, and the opposite case.

Remark 13.2. Problem 2 may be considered as generalization of Problem 1 to the case when the zeroes of $S$ and of $S_{i}^{\prime}$ are not numbered.

Remark 13.3. In both settings the $\mathcal{H}\left(\alpha_{i}^{\prime}\right)$ may be disconnected. We could also specify the connected component of the $\mathcal{H}\left(\alpha_{i}^{\prime}\right)$ when this occurs. We prefer the setting where this data is not specified.

Consider the natural action of the cyclic group of order $p$ on the elements of the assignment organized in a cyclic order. If it has a nontrivial stabilizer we denote it by the same symbol $\Gamma$ as before. We get a symmetry of order $|\Gamma|$ which we again call the stratum interchange. Note that this symmetry might be different in the settings of Problem 1 and Problem 2.

Example 13.4. The assignment

$$
(\overline{1}, \overline{1}, 2) \rightarrow(\overline{1}, \overline{1}) \Rightarrow(\overline{1}, \overline{1}, 2) \rightarrow(\overline{1}, \overline{1}) \Rightarrow
$$

does not possess the stratum interchange symmetry in the setting of Problem 1 since the unchanged zeroes of order 2 are named, and so they identify the components of the type $(2,1,1)$. However, in the setting of Problem 2 this configuration has the stratum interchange symmetry of order 2 .

The assignment

$$
(\overline{1}, \overline{1}) \rightarrow(\overline{1}, \overline{1}) \Rightarrow(\overline{1}, \overline{1}) \rightarrow(\overline{1}, \overline{1}) \Rightarrow
$$

possesses the stratum interchange symmetry of order 2 in both settings.

Recall that for saddle connections joining distinct zeroes we also might also have the $\gamma \rightarrow-\gamma$ symmetry. It could appear only when $m_{1}=m_{2}$, for in this case we have no invariant way of choosing the orientation of the saddle connection. When we have only saddle connections joining a zero to itself, there is no geometric way of choosing the orientation of saddle connections. The two different choices of the orientation give two decompositions of $S$. Thus we get the action of the group $\mathbb{Z} / 2 \mathbb{Z}$ on the collection of assignments. We call this action the $\gamma \rightarrow-\gamma$ symmetry. This action is easily described in combinatorial terms: one has to change the cyclic order in the assignment to the opposite one, as well as the order in each pair $\left(a_{i}^{\prime}, a_{i}^{\prime \prime}\right)$ and $\left(b_{j}^{\prime}, b_{j}^{\prime \prime}\right)$. Some assignments may stay invariant under this operation. In this case we say that they have $\gamma \rightarrow-\gamma$ symmetry. This property also depends whether we consider the assignments in the setting of Problem 1 or of Problem 2. 
Example 13.5. The first assignment in Example 13.4 does not have the $\gamma \rightarrow-\gamma$ symmetry; the second one does.

An assignment of multiplicity 1 has $\gamma \rightarrow-\gamma$ symmetry if and only if $a^{\prime}=a^{\prime \prime}$ when we apply the figure eight construction and if and only if $b^{\prime}=b^{\prime \prime}$ when we the apply the creating a hole construction.

The assignment

$$
(\overline{2}, \overline{2}, 2) \Rightarrow(\overline{2}, \overline{2}, 2) \Rightarrow
$$

has the $\gamma \rightarrow-\gamma$ symmetry in both settings, while the assignment

$$
(\overline{2}, \overline{2}, 2) \Rightarrow(\overline{2}, \overline{2}, 2) \Rightarrow(\overline{2}, \overline{2}, 2) \Rightarrow
$$

has the $\gamma \rightarrow-\gamma$ symmetry only in the setting of Problem 2 .

Remark 13.6. In describing the construction $\left(S^{\prime}, J, a_{i}^{\prime}, a_{i}^{\prime \prime}, b_{i}^{\prime}, b_{i}^{\prime \prime}, \tau, \gamma, h, t\right) \rightarrow S$ one can consider all possible assignments, and then take into account corresponding symmetries, or one can deal with the classes, eliminating the symmetry whenever it is possible.

We have chosen the second way. For example, the assignment $(\overline{1}, \overline{0}, 1) \rightarrow$ (see Section 13.4) is symmetric to the assignment $(\overline{0}, \overline{1}, 1) \rightarrow$ by the $\gamma \rightarrow-\gamma$ symmetry.

13.3. Combinatorial Factor, Connected Strata. We now compute the combinatorial factor $M$, which computes for fixed data $\left(S^{\prime}, J, a_{i}^{\prime}, a_{i}^{\prime \prime}, b_{k}^{\prime}, b_{k}^{\prime \prime}, \gamma, h, t\right)$ the number of $S=\sigma\left(S^{\prime}\right)$ that can be built. We start with the setting of Problem 1 in section 13.2 We first suppose that the stratum $\mathcal{H}(\alpha)$ is connected.

Consider a surface $S_{i}^{\prime}$ such that $z_{i}=w_{i}$ is a zero of order $a_{i}=a_{i}^{\prime}+a_{i}^{\prime \prime}$. We apply the figure eight construction by breaking $z_{i}=w_{i}$ into zeroes of orders $a_{i}^{\prime}$ and $a_{i}^{\prime \prime}$. There are $a_{i}+1$ ways of doing this, see section 12.1 The corresponding boundary components are denoted $\gamma_{i}^{\prime}, \gamma_{i}^{\prime \prime}$. The component $\gamma_{i}^{\prime}$ is attached to $S_{i+1}$ (directly or by means of a cylinder depending on whether or not $i \in J$ ); the component $\gamma_{i}^{\prime \prime}$ is attached to $S_{i-1}$ (directly or by means of a cylinder depending on whether or not $i-1 \in J)$.

If $z_{k} \neq w_{k}$ we perform the construction of creating a pair of holes. The orders of the zeroes are $b_{k}^{\prime \prime}$ and $b_{k}^{\prime}$ correspondingly, so there are $b_{k}^{\prime \prime}+1$ ways of choosing the vector $\gamma$ at $z_{k}$ and $b_{k}^{\prime}+1$ ways of choosing the vector $\gamma$ at $w_{i}$ so this gives $\left(b_{k}^{\prime}+1\right)\left(b_{k}^{\prime \prime}+1\right)$ choices.

We may have the stratum interchange symmetry or (and) $\gamma \rightarrow-\gamma$ symmetry, see section 13.2 Thus the resulting combinatorial factor in the setting of Problem 1 in section 13.2 equals

$$
c=\frac{1}{\left|\Gamma_{-}\right|} \cdot \frac{1}{|\Gamma|} \cdot \prod_{\substack{1 \leq i \leq p \\ z i=w_{i}}}\left(a_{i}+1\right) \cdot \prod_{\substack{1 \leq k \leq p \\ z_{k} \neq w_{k}}}\left(b_{k}^{\prime}+1\right)\left(b_{k}^{\prime \prime}+1\right)
$$

We now compute the combinatorial factor in the setting of Problem 2 in Section 13.2 For each integer $m$, let $o(m)$ denote its multiplicity. For each $i, i=$ $1, \ldots, p$, let $o_{i}(m)$ denote the multiplicity of $m$ in $\alpha_{i}^{\prime}$.

Consider an integer $m$ different from the order of any newborn zero on $S$ (see formulae 29. 30] 31), and different from any $a_{i}$ or $b_{k}^{\prime}, b_{k}^{\prime \prime}$. Since all the zeroes $z_{1}, z_{2}, \ldots, z_{l}$ of $S$ are numbered, the number of ways to arrange $o(m)$ zeroes of order $m$ into groups of $o_{1}(m), \ldots, o_{p}(m)$ zeroes equals

$$
\frac{o(m) !}{\prod_{i=1}^{p} o_{i}(m) !}
$$


where we let $0 !=1$ by convention.

Let now $s \geq 1$ be the number of the newborn zeroes in $S$ of order $m$, and suppose that $m$ is different from any $a_{i}, b_{k}^{\prime}, b_{k}^{\prime \prime}$. Now we have to choose $s$ of $o(m)$ numbered zeroes to be the newborn ones, and then we have to arrange the remaining $o(m)-s$ ordered zeroes into groups of $o_{1}(m), \ldots, o_{p}(m)$ zeroes. For such $m$ we get the factor

$$
o(m)(o(m)-1) \cdots(o(m)-s+1) \cdot \frac{(o(m)-s) !}{\prod_{i=1}^{p} o_{i}(m) !}=\frac{o(m) !}{\prod_{i=1}^{p} o_{i}(m) !}
$$

which coincides with the previous one. Hence, we do not need to distinguish this case from the previous one.

If $m$ is different from any $b_{k}^{\prime}, b_{k}^{\prime \prime}$, but is equal to some $a_{i}$, then there are only $o_{i}(m)-1$ zeroes of order $m$ on $S_{i}^{\prime}$ inherited from $S$. Thus the corresponding factor in the denominator in the formula above equals $\left(o_{i}(m)-1\right)$ !. Multiplying numerator and denominator by $o_{i}(m)$ we get the following factor for such $m$ :

$$
\frac{o(m) !}{\prod_{i=1}^{p} o_{i}(m) !} \cdot \prod_{\substack{1 \leq i \leq p \\ a_{i}=m \\ z_{i}=w_{i}}} o_{i}(m)
$$

We have to make a similar correction for those $m$ which coincide with some $b_{k}^{\prime}$ or $b_{k}^{\prime \prime}$. In the case when $m$ equals one of the $b_{k}^{\prime} \neq b_{k}^{\prime \prime}$ the correction is completely analogous to the previous one. In the case when for some $k$ we have $m=b_{k}^{\prime}=b_{k}^{\prime \prime}$, we get only $o(m)-2$ zeroes of order $m$ on $S_{k}^{\prime}$, inherited from $S$. Thus the corresponding factor in the denominator in the formula above equals $\left(o_{k}(m)-2\right)$ !. Multiplying numerator and denominator by $o_{k}(m)\left(o_{k}(m)-1\right)$ and collecting all the correction terms we finally get the following combinatorial factor for the setting of Problem 2 in section 13.2

$$
\begin{aligned}
M= & \frac{1}{\left|\Gamma_{-}\right|} \cdot \frac{1}{|\Gamma|} \cdot \prod_{m \in \alpha}\left(\frac{o(m) !}{\prod_{i=1}^{p} o_{i}(m) !}\right) \cdot \\
& \cdot \prod_{\substack{1 \leq i \leq p \\
z_{i}=w_{i} \\
a_{i} \neq 0}} o_{i}\left(a_{i}\right) \cdot \prod_{\substack{1 \leq k \leq p \\
z_{k} \neq w_{k} \\
b_{k}^{\prime} \neq b_{k}^{\prime \prime} \\
b_{k}^{\prime} \neq 0}} o_{k}\left(b_{k}^{\prime}\right) \cdot \prod_{\substack{1 \leq k \leq p \\
z_{k} \neq w_{k} \\
b_{k}^{\prime} \neq b_{k}^{\prime \prime} \\
b_{k}^{\prime \prime} \neq 0}} o_{k}\left(b_{k}^{\prime \prime}\right) \cdot \prod_{\substack{1 \leq k \leq p \\
z_{k} \neq w_{k} \\
b_{k}^{\prime}=b_{k}^{\prime \prime} \neq 0}} o_{k}\left(b_{k}^{\prime}\right)\left(o_{k}\left(b_{k}^{\prime}\right)-1\right) \cdot \\
& \cdot \prod_{\substack{1 \leq i \leq p \\
z_{i}=w_{i}}}\left(a_{i}+1\right) \cdot \prod_{\substack{1 \leq k \leq p \\
z_{k} \neq w_{k}}}\left(b_{k}^{\prime}+1\right)\left(b_{k}^{\prime \prime}+1\right)
\end{aligned}
$$

Note that in general the symmetry groups $\Gamma$ and $\Gamma_{-}$corresponding to possible stratum interchange symmetry and to $\gamma \rightarrow-\gamma$ symmetry are different in Problems 1 and 2 , see Section 13.2

There will also be additional factors coming from the spin structures and the hyperelliptic strata. We shall discuss these separately.

We finally get the following expression for the constant $c$ in the setting where the zeroes of $S$ are not numbered (see Problem 2 in Section 13.2):

Formula 13.1. The list of possible configurations of homologous saddle connections joining zeroes to themselves is the same for almost all surfaces in any connected stratum $\mathcal{H}(\alpha)$. The possible configurations are described in section 12.3. The constant in the quadratic asymptotics for the number of saddle connections with associated data $\left(J, a_{i}^{\prime}, a_{i}^{\prime \prime}, b_{k}^{\prime}, b_{k}^{\prime \prime}\right)$ is presented by the following formula: 


$$
\begin{gathered}
c=\frac{1}{\left|\Gamma_{-}\right|} \cdot \frac{1}{|\Gamma|} \cdot \prod_{\substack{m \in \alpha \\
\prod_{i \leq i \leq p}=w_{i} \\
a_{i} \in \alpha}}\left(\frac{o(m) !}{\prod_{i=1}^{p} o_{i}(m) !}\right) \cdot \\
\qquad \prod_{\substack{1 \leq k \leq p \\
z_{k} \neq w_{k} \\
b_{k}^{\prime} \neq b_{k}^{\prime \prime} \\
b_{k}^{\prime} \in \alpha}} o_{k}\left(b_{k}^{\prime}\right) \cdot \prod_{\substack{1 \leq k \leq p \\
z_{k} \neq w_{k} \\
b_{k}^{\prime} \neq b_{k}^{\prime \prime} \\
b_{k}^{\prime \prime} \in \alpha}} o_{k}\left(b_{k}^{\prime \prime}\right) \cdot \prod_{\substack{1 \leq k \leq p \\
z_{k} \neq w_{k} \\
b_{k}^{\prime}=b_{k}^{\prime \prime} \in \alpha}} o_{k}\left(b_{k}^{\prime}\right)\left(o_{k}\left(b_{k}^{\prime}\right)-1\right) \cdot \\
\cdot \prod_{\substack{1 \leq i \leq p \\
z_{i}=w_{i}}}\left(a_{i}+1\right) \cdot \prod_{\substack{1 \leq k \leq p \\
z_{k} \neq w_{k}}}\left(b_{k}^{\prime}+1\right)\left(b_{k}^{\prime \prime}+1\right) \cdot \\
\cdot \frac{1}{2^{p-1}} \cdot \frac{\prod_{i=1}^{p}\left(\frac{d_{i}}{2}-1\right) !}{\left(\frac{d}{2}-2\right) !} \cdot \frac{\prod_{i=1}^{p} \operatorname{Vol}\left(\mathcal{H}_{1}\left(\alpha_{i}^{\prime}\right)\right)}{\operatorname{Vol}\left(\mathcal{H}_{1}(\alpha)\right)}
\end{gathered}
$$

\subsection{Examples: Constants for Strata in Genus 3. Stratum $\mathcal{H}(\mathbf{3}, \mathbf{1})$}

\section{$\rightarrow(\overline{\mathbf{1}}, \overline{\mathbf{0}}, \mathbf{1}) \rightarrow$}

The first possibility is multiplicity 1 with no cylinder. After degeneration we get a genus 2 surface with a distinguished simple zero, a marked point and another simple zero. We have $d_{1}=12$. We employ the parallelogram construction to the distinguished simple zero and marked point producing two circles which we glue directly. Since $1=b_{1}^{\prime} \neq b_{1}^{\prime \prime}=0$ we do not have the $\gamma \rightarrow-\gamma$ symmetry. Thus $M=\left(b_{1}^{\prime}+1\right)\left(b_{1}^{\prime \prime}+1\right)=2$. We have

$$
c=2 \cdot \frac{(5+1-1) !}{(7-2) !} \cdot \frac{\operatorname{Vol}\left(\mathcal{H}_{1}(1,1)\right)}{\operatorname{Vol}\left(\mathcal{H}_{1}(3,1)\right)}=\frac{105}{16} \cdot \frac{1}{\zeta(2)} \approx 3.990
$$

$\Rightarrow(\overline{1+0}, 1) \Rightarrow$

The next possibility is multiplicity 1 with a cylinder where the other side also returns to the zero of order 3 . After degeneration we get a surface of genus 2 with a distinguished simple zero on which we perform the figure eight construction, gluing in a cylinder. Since $1=a_{1}^{\prime} \neq a_{1}^{\prime \prime}=0$ we do not have the $\gamma \rightarrow-\gamma$ symmetry. Thus $M=\left(a_{1}+1\right)=2$. Now $d_{1}=10$ so

$$
c=2 \cdot \frac{(5-1) !}{(7-2) !} \cdot \frac{\operatorname{Vol}\left(\mathcal{H}_{1}(1,1)\right)}{\operatorname{Vol}\left(\mathcal{H}_{1}(3,1)\right)}=\frac{21}{16} \cdot \frac{1}{\zeta(2)} \approx 0.7979
$$

$\Rightarrow(\overline{2}, \overline{0}) \Rightarrow$

If the other side returns to the simple zero, then after degeneration, we get a genus 2 surface with a double zero and a marked point on which we perform the creating a hole construction. Again $d_{1}=10$. Since $2=b_{1}^{\prime} \neq b_{1}^{\prime \prime}=0$ we do not have the $\gamma \rightarrow-\gamma$ symmetry. Thus $M=\left(b_{1}^{\prime}+1\right)\left(b_{1}^{\prime \prime}+1\right)=3$. We have

$$
c=3 \cdot \frac{(4+1-1) !}{(7-2) !} \cdot \frac{\operatorname{Vol}\left(\mathcal{H}_{1}(2)\right)}{\operatorname{Vol}\left(\mathcal{H}_{1}(3,1)\right)}=\frac{567}{256} \cdot \frac{1}{\zeta(2)} \approx 1.346
$$

$\Rightarrow(\overline{\mathbf{0}+\mathbf{0}}) \rightarrow(\overline{\mathbf{0}}, \overline{\mathbf{0}}) \Rightarrow$

Now we consider multiplicity 2 in this stratum. Then we have a cylinder whose boundary components are curves returning to the zero of order 3 and zero of order 
one. The homologous curve returns to the zero of order 3. The degenerating surfaces are tori, one with a single marked point, the other with 2 marked points. On the torus with one point, we perform the figure eight construction and on the other the creating a hole construction. We glue in one cylinder and one pair of circles is glued directly.

There is no $\gamma \rightarrow-\gamma$ symmetry in this case.

In this case we get

$$
c=1 \cdot \frac{1}{2^{2-1}} \cdot \frac{(3-1) ! \cdot(2-1) !}{(7-2) !} \cdot \frac{\left.\operatorname{Vol}\left(\mathcal{H}_{1}(0)\right)\right)^{2}}{\operatorname{Vol}\left(\mathcal{H}_{1}(3,1)\right)}=\frac{105}{256} \cdot \frac{1}{\zeta(2)} \approx 0.2493
$$

\section{Stratum $\mathcal{H}(2,1,1)$}

$\rightarrow(\mathbf{1}, \mathbf{1}, \overline{\mathbf{0}}, \overline{\mathbf{0}}) \rightarrow$

The multiplicity 1 case with no cylinder degenerates to a genus 2 surface with 2 marked points. We apply the parallelogram construction to the marked points, gluing the circles directly to each other. There is the $\gamma \rightarrow-\gamma$ symmetry.

We get

$$
c=\frac{1}{2} \cdot \frac{(5+2-1) !}{(8-2) !} \cdot \frac{\operatorname{Vol}\left(\mathcal{H}_{1}(1,1)\right)}{\operatorname{Vol}\left(\mathcal{H}_{1}(2,1,1)\right)}=\frac{7}{3} \cdot \frac{1}{\zeta(2)} \approx 1.418
$$

$\Rightarrow(1,1, \overline{\mathbf{0}+\mathbf{0}}) \Rightarrow$

For the case of a cylinder with multiplicity 1 where the other side returns to the double zero the degenerating surface has genus 2 with a single marked point. We apply the figure eight construction at the marked point. We have the $\gamma \rightarrow-\gamma$ symmetry.

$$
c=\frac{1}{2} \cdot \frac{(5+1-1) !}{(8-2) !} \cdot \frac{\operatorname{Vol}\left(\mathcal{H}_{1}(1,1)\right)}{\operatorname{Vol}\left(\mathcal{H}_{1}(2,1,1)\right)}=\frac{7}{18} \cdot \frac{1}{\zeta(2)} \approx 0.2364
$$

$\Rightarrow(\mathbf{1}, \overline{\mathbf{1}}, \overline{\mathbf{0}}) \Rightarrow$

If the other side of the cylinder returns to a simple zero, then after degeneration we get a surface of genus 2 with a marked point and a simple zero (as well as another simple zero on the surface). We apply the creating a pair of holes construction at the marked point and a zero gluing in a cylinder. Now we have $b_{1}^{\prime}=0, b_{1}^{\prime \prime}=1$. Since $b_{1}^{\prime} \neq b_{2}^{\prime \prime}$ we do not have a $\gamma \rightarrow-\gamma$ symmetry.

$$
c=4 \cdot \frac{(5+1-1) !}{(8-2) !} \cdot \frac{\operatorname{Vol}\left(\mathcal{H}_{1}(1,1)\right)}{\operatorname{Vol}\left(\mathcal{H}_{1}(2,1,1)\right)}=\frac{28}{9} \cdot \frac{1}{\zeta(2)} \approx 1.891
$$

$\Rightarrow(2, \overline{0}, \overline{0}) \Rightarrow$

If the cylinder joins two simple zeroes, then after degeneration we get a surface of genus 2 with a zero of order 2 and two marked points. We apply the parallelogram construction at the marked points. We have $b_{1}^{\prime}=b_{1}^{\prime \prime}=0$. Here we have $\gamma \rightarrow-\gamma$ symmetry.

$$
c=1 \cdot \frac{(4+2-1) !}{(8-2) !} \cdot \frac{\operatorname{Vol}\left(\mathcal{H}_{1}(2)\right)}{\operatorname{Vol}\left(\mathcal{H}_{1}(2,1,1)\right)}=\frac{7}{8} \cdot \frac{1}{\zeta(2)} \approx 0.5319
$$

$\Rightarrow(\overline{\mathbf{0}}, \overline{\mathbf{0}}) \rightarrow(\overline{\mathbf{0}}, \overline{\mathbf{0}}) \Rightarrow$

There are two multiplicity $p=2$ cases. The first is if there is a cylinder with boundary curves returning to the simple zeroes and a homologous curve returning 
to the double zero. We get 2 tori each with 2 marked points so $p=2, d_{1}=d_{2}=6$. We perform the parallelogram construction on each and then glue in one cylinder and glue two circles directly. We have the $\gamma \rightarrow-\gamma$ symmetry, and we do not have a stratum interchange.

$$
c=1 \cdot \frac{1}{2^{2-1}} \cdot \frac{(3-1) ! \cdot(3-1) !}{(8-2) !} \cdot \frac{\operatorname{Vol}\left(\mathcal{H}_{1}(0)\right)^{2}}{\operatorname{Vol}\left(\mathcal{H}_{1}(2,1,1)\right)}=\frac{7}{36} \cdot \frac{1}{\zeta(2)} \approx 0.1182
$$

$\Rightarrow(\overline{0}, \overline{0}) \Rightarrow(\overline{0+0}) \Rightarrow$

The second case is if there are 2 cylinders. In that case, after degeneration, we get a torus with 2 marked points and a torus with one marked point. We apply the figure eight construction to the torus with one marked point and the parallelogram construction to the other. We then glue in cylinders to each pair of circles. Again we have the $\gamma \rightarrow-\gamma$ symmetry, and we do not have a stratum interchange.

$$
c=1 \cdot \frac{1}{2^{2-1}} \cdot \frac{(3-1) ! \cdot(2-1) !}{(8-2) !} \cdot \frac{\operatorname{Vol}\left(\mathcal{H}_{1}(0)\right)^{2}}{\operatorname{Vol}\left(\mathcal{H}_{1}(2,1,1)\right)}=\frac{7}{72} \cdot \frac{1}{\zeta(2)} \approx 0.05910
$$

13.5. Principal Stratum. Since any figure eight construction produces a "newborn" zero of order at least two, here we cannot have any figure eight constructions. All the surfaces $S_{i}^{\prime}$, belong to the principal strata in lower genera $g_{i}$; there are a pair of marked points $z_{i} \neq w_{i}$ on each surface, and we always apply the parallelogram construction with $b_{i}^{\prime}=b_{i}^{\prime \prime}=0$. All the curves $\gamma_{i}$ bound cylinders.

13.5.1. Saddle Connections of Multiplicity 1. In multiplicity one there is a single surface $S^{\prime} \in \mathcal{H}\left(\alpha^{\prime}\right)$ of genus $g-1$ and $S^{\prime}$ has two marked points $z \neq w$. We recover $S$ from $S^{\prime}$ by applying the parallelogram construction followed by gluing in a cylinder; $\alpha^{\prime}$ is the partition of $2(g-1)-2$ into ones.

We always have the $\gamma \rightarrow-\gamma$ symmetry, so $\left|\Gamma_{-}\right|=2$ in all cases. We use the setting of Problem 2 of section 13.2 The combinatorial constant is

$$
M=\frac{1}{2} \cdot \frac{(2 g-2) !}{(2 g-4) !} \cdot(0+1)(0+1)
$$

Applying (32) we get the following value for the constant $c$ :

$$
c=\frac{(2 g-2)(2 g-3)}{2} \cdot \frac{((4 g-3-2)-1) !}{((4 g-3)-2) !} \cdot \frac{\operatorname{Vol}\left(\mathcal{H}_{1}\left(\alpha^{\prime}\right)\right)}{\operatorname{Vol}\left(\mathcal{H}_{1}(\alpha)\right)}
$$

Thus, finally,

$$
\left.c=\frac{(g-1)(2 g-3)}{4 g-5} \cdot \frac{\operatorname{Vol}\left(\mathcal{H}_{1}(\overbrace{1, \ldots, 1}^{2 g-4})\right.}{\operatorname{Vol}\left(\mathcal{H}_{1}(\underbrace{1, \ldots, 1}_{2 g-2})\right.}\right)
$$

In the table below we present the values of the constant for saddle connections of multiplicity one joining a zero to itself, for flat surfaces living in the principal strata in small genera. 
TABLe 7. Principal stratum $\mathcal{H}(1, \ldots, 1)$; values of the constants in the quadratic asymptotics for the number of closed geodesics of multiplicity one.

\begin{tabular}{|l|c|c|c|c|c|c|c|}
\hline & $g=2$ & $g=3$ & $g=4$ & $g=5$ & $g=6$ & $g=7$ & $g=8$ \\
\hline$c \cdot \zeta(2)=$ & $\frac{5}{2}$ & $\frac{36}{7}$ & $\frac{3150}{377}$ & $\frac{274456}{23357}$ & $\frac{250153470}{16493303}$ & $\frac{6531347988}{351964697}$ & $\frac{8007196856750}{365342975469}$ \\
\hline$c \approx$ & 1.51982 & 3.12648 & 5.07950 & 7.14344 & 9.22041 & 11.2812 & 13.3239 \\
\hline
\end{tabular}

13.5.2. Higher Multiplicity in the Principle Stratum. The surface $S$ breaks up into a union of $p$ surfaces $S_{i}$ of genera $g_{i}, i=1, \ldots, p$. Each $S_{i}$ has only simple zeroes and a pair of marked points $z_{i}$ and $w_{i}$. We reconstruct $S$ by the creating a pair of holes construction at the marked points $z_{i}$ and $w_{i}$, and then by gluing in cylinders $C_{i}$ to connect the boundary circles.

For multiplicity two assignments there is always a factor of $1 / 2$ due to the symmetry $\gamma \rightarrow-\gamma$. For higher multiplicities an assignment has the $\gamma \rightarrow-\gamma$ symmetry if and only if the corresponding cyclic ordering $g_{1} \rightarrow \cdots \rightarrow g_{p} \rightarrow$ is invariant under reversing arrows (up to a cyclic shift of the entries). The assignment has the stratum interchange symmetry if and only if the corresponding cyclic ordering $g_{1} \rightarrow \cdots \rightarrow g_{p} \rightarrow$ has translational symmetry. The resulting combinatorial factor is

$$
M=\frac{1}{\left|\Gamma_{-}\right| \cdot|\Gamma|} \frac{(2 g-2) !}{\prod_{i=1}^{p}\left(2 g_{i}-2\right) !}
$$

Let $\alpha_{i}$ denote the partition of $2 g_{i}-2$ into ones. Applying our formula with $M$ as above, we get

$$
c=\frac{M}{2^{p-1}(4 g-5) ! \operatorname{Vol}\left(\mathcal{H}_{1}(\alpha)\right)} \prod_{i=1}^{p}\left(4 g_{i}-2\right) ! \operatorname{Vol}\left(\mathcal{H}_{1}\left(\alpha_{i}\right)\right)
$$

To complete this section we present some examples of these computations.

Example 13.7. Stratum $\mathcal{H}(\mathbf{1}, \mathbf{1}, \mathbf{1}, \mathbf{1})$. In the stratum $\mathcal{H}(1,1,1,1)$ in genus 3 , the only higher multiplicity is $p=2$ so $S_{1}^{\prime}, S_{2}^{\prime}$ have genus 1 . We have the stratum interchange symmetry factor of $|\Gamma|=2$; as in all these cases we also have the $\gamma \rightarrow-\gamma$ symmetry, $\left|\Gamma_{-}\right|=2$. Hence we have the combinatorial factor

Thus

$$
M=\frac{1}{2} \cdot \frac{1}{2} \cdot \frac{4 !}{0 ! \cdot 0 !}=6
$$

$$
c=6 \cdot \frac{1}{2^{2-1}} \cdot \frac{2 ! \cdot 2 !}{7 !} \cdot \frac{\left(\operatorname{Vol}\left(\mathcal{H}_{1}(0)\right)\right)^{2}}{\operatorname{Vol}\left(\mathcal{H}_{1}(1,1,1,1)\right)}=\frac{3}{14} \cdot \frac{1}{\zeta(2)} \approx 0.1303
$$

Example 13.8. Stratum $\mathcal{H}(\mathbf{1}, \mathbf{1}, \mathbf{1}, \mathbf{1}, \mathbf{1}, \mathbf{1})$. Now $g=4$, and we have two different nontrivial partitions of $g-1=3$ arranged in a cyclic order: in multiplicity 2, a surface of genus 2 and a surface of genus 1 and in multiplicity 3 yielding three tori. In the first case we do not have the stratum interchange symmetry, $|\Gamma|=1$. Hence we get the following combinatorial factor

$$
M=\frac{1}{2} \cdot \frac{6 !}{0 ! \cdot 2 !}
$$


Thus

$$
c=\frac{6 !}{4} \cdot \frac{1}{2^{2-1}} \cdot \frac{2 ! \cdot 6 !}{11 !} \cdot \frac{\operatorname{Vol}\left(\mathcal{H}_{1}(0)\right) \operatorname{Vol}\left(\mathcal{H}_{1}(1,1)\right)}{\operatorname{Vol}\left(\mathcal{H}_{1}(1,1,1,1,1,1)\right)}=\frac{90}{377} \cdot \frac{1}{\zeta(2)} \approx 0.1451
$$

In the second case we have the stratum interchange symmetry, $|\Gamma|=3$. Hence we get the following combinatorial factor

$$
M=\frac{1}{2} \cdot \frac{1}{3} \cdot \frac{6 !}{0 ! \cdot 0 ! \cdot 0 !}=5 !
$$

Thus

$$
c=5 ! \cdot \frac{1}{2^{3-1}} \cdot \frac{2 ! \cdot 2 ! \cdot 2 !}{11 !} \cdot \frac{\left(\operatorname{Vol}\left(\mathcal{H}_{1}(0)\right)\right)^{3}}{\operatorname{Vol}\left(\mathcal{H}_{1}(1,1,1,1,1,1)\right)}=\frac{5}{754} \cdot \frac{1}{\zeta(2)} \approx 0.004031
$$

Example 13.9. Stratum $\mathcal{H}(\mathbf{1}, \mathbf{1}, \mathbf{1}, \mathbf{1}, \mathbf{1}, \mathbf{1}, \mathbf{1}, \mathbf{1})$. Proceeding to genus 5 we get the following nontrivial partitions of $g-1=4$ arranged in a cyclic order: in multiplicity 2 giving a surface of genus 1 and one of genus 3; in multiplicity 2 yielding two surfaces of genus 2 , in multiplicity 3 yielding two tori and a genus 2 surface and in multiplicity 4 yielding 4 tori.

In the first case we do not have any stratum interchange symmetry, $|\Gamma|=1$. Hence we get the following combinatorial factor

Thus

$$
M=\frac{1}{2} \cdot \frac{8 !}{0 ! \cdot 4 !}
$$

$$
c=\frac{8 !}{2 \cdot 4 !} \cdot \frac{1}{2^{2-1}} \cdot \frac{2 ! \cdot 10 !}{15 !} \cdot \frac{\operatorname{Vol}\left(\mathcal{H}_{1}(0)\right) \operatorname{Vol}\left(\mathcal{H}_{1}(1,1,1,1)\right)}{\operatorname{Vol}\left(\mathcal{H}_{1}(1,1,1,1,1,1,1,1)\right)}=\frac{4200}{23357} \cdot \frac{1}{\zeta(2)} \approx 0.1093
$$

For the other multiplicity 2 case $\alpha_{1}^{\prime}=\alpha_{2}^{\prime}=(1,1)$. We have the stratum interchange symmetry, $|\Gamma|=2$. Hence we get the following combinatorial factor

$$
M=\frac{1}{2} \cdot \frac{1}{2} \cdot \frac{8 !}{2 ! \cdot 2 !}
$$

Thus

$$
c=\frac{8 !}{16} \cdot \frac{1}{2^{2-1}} \cdot \frac{6 ! \cdot 6 !}{15 !} \cdot \frac{\left(\operatorname{Vol}\left(\mathcal{H}_{1}(1,1)\right)\right)^{2}}{\operatorname{Vol}\left(\mathcal{H}_{1}(1,1,1,1,1,1,1,1)\right)}=\frac{720}{23357} \cdot \frac{1}{\zeta(2)} \approx 0.01874
$$

For the third case we do not have any stratum interchange symmetry, $|\Gamma|=1$. Hence we get the following combinatorial factor

$$
M=\frac{1}{2} \cdot \frac{8 !}{0 ! \cdot 0 ! \cdot 2 !}
$$

Thus

$$
c=\frac{8 !}{4} \cdot \frac{1}{2^{3-1}} \cdot \frac{2 ! \cdot 2 ! \cdot 6 !}{15 !} \cdot \frac{\left(\operatorname{Vol}\left(\mathcal{H}_{1}(0)\right)\right)^{2} \operatorname{Vol}\left(\mathcal{H}_{1}(1,1)\right)}{\operatorname{Vol}\left(\mathcal{H}_{1}(1,1,1,1,1,1,1,1)\right)}=\frac{120}{23357} \cdot \frac{1}{\zeta(2)} \approx 0.003123
$$

In the last case we have stratum a interchange symmetry, $|\Gamma|=4$. Hence we get the following combinatorial factor

$$
M=\frac{1}{2} \cdot \frac{1}{4} \cdot \frac{8 !}{0 ! \cdot 0 ! \cdot 0 ! \cdot 0 !}=7 !
$$

Thus

$$
c=7 ! \cdot \frac{1}{2^{4-1}} \cdot \frac{2 ! \cdot 2 ! \cdot 2 ! \cdot 2 !}{15 !} \cdot \frac{\left(\operatorname{Vol}\left(\mathcal{H}_{1}(0)\right)\right)^{4}}{\operatorname{Vol}\left(\mathcal{H}_{1}(1,1,1,1,1,1,1,1)\right)}=\frac{5}{46714} \cdot \frac{1}{\zeta(2)} \approx 0.00006507
$$




\section{Strata that are not Connected}

14.1. Parity of the Spin Structure of the Compound Surface. Consider a flat surface $S$ constructed in the previous section. Throughout this section we suppose that all zeroes of $\omega$ are of even orders, so the resulting flat surface $S$ has a spin structure. In this section we discuss the conditions on components $S_{i}^{\prime}$, and on the gluing rules between them which allow us to obtain a surface $S$ with even $\alpha$. We also compute the parity of the spin structure of $S$. This will allow us to find constants in the case of components with spin structures.

Lemma 14.1. Let a nondegenerate flat surface $S$ be obtained from a collection $S_{i}^{\prime}$ by applying the figure eight and parallelogram constructions. The resulting flat surface $S$ has zeroes of even orders if and only if the following conditions are valid:

- All zeroes of those surfaces $S_{i}^{\prime}$ to which we apply the figure eight construction have even orders;

- If there is at least one surface to which we apply the parallelogram construction, then there is the following additional alternative. Either

- all zeroes of all surfaces are of even order and every newborn zero of $S$ is of type III;

or

- the orders $b_{k}^{\prime}, b_{k}^{\prime \prime}$ of all zeroes $z_{k} \neq w_{k}$ to which we apply the creating a pair of holes construction are odd while all the other zeroes of any $S_{i}$ are even.

Proof. First note that all zeroes inherited by $S$ from $S_{i}^{\prime}$ without changes are of even orders. Thus, if we apply to $S_{i}^{\prime}$ the figure eight construction, than all the zeroes of $S_{i}^{\prime}$ different from $z_{i}$ must have even orders. Since the total sum of orders equals $2 g_{i}+2$, it means that $a_{i}$ - the order of $z_{i}$ - is even as well.

Suppose now that there is a surface $S_{k}^{\prime}$ to which we apply the creating a pair of holes construction with at least one of $b_{k}^{\prime}, b_{k}^{\prime \prime}$ even. Without loss of generality we may assume that $b_{k}^{\prime}$ is even. By the same arguments as above all zeroes of $S_{k}^{\prime}$ different from $z_{k}$ and $w_{k}$ have even orders. Since the total sum $2 g_{k}+2$ of all orders is even, the remaining zero has even order $b_{k}^{\prime \prime}$ as well.

Consider the newborn zero of $S$ induced from $w_{k}^{\prime}$. It is of one of the types II or III. If it were of type II, then by (30) it would have odd order. Thus this newborn zero is of the type III. Let $S_{k+s+1}^{\prime}$ be the surface, to which we apply creating a pair of holes construction, at the opposite end of the chain from $S_{k}^{\prime}$. By (31) $b_{k+s+1}^{\prime}$ is even. Repeating our arguments we show that all zeroes of all $S_{i}^{\prime}$ are even, and that all newborn zeroes are of the type III.

The remaining part of the alternative now becomes obvious.

Let us calculate the parity of the spin structure of the resulting surface $S$ in all possible cases described above.

Lemma 14.2. Suppose that we use only figure eight constructions to glue a nondegenerate surface $S$. Suppose that all $\alpha_{i}$ are even. The parity of the spin structure of the resulting surface $S$ is defined by the parities of the spin structures of the components, and by the parities of the numbers $a_{i}^{\prime}$ in the following way:

$$
\phi(S)=1+\sum_{i=1}^{p} \phi\left(S_{i}^{\prime}\right)+\sum\left(a_{i}^{\prime}+1\right) \quad(\bmod 2)
$$


Proof. For each surface $S_{i}$ consider a collection of $2 g_{i}$ smooth simple closed curves on $S_{i}^{\prime}$ representing a canonical basis of cycles. As usual we may assume that the curves stay outside a neighborhood of the zeroes and the marked points of $S_{i}^{\prime}$.

We may construct now the following canonical basis of cycles on the resulting surface $S$. Let $g$ be the genus of $S$. First take all the cycles represented by the basic curves on components. They give us $g-1$ pairs of cycles. By construction they form incomplete canonical basis of cycles on $S$.

Since we are using only figure eight constructions we have to use at least one cylinder to obtain a nondegenerate surface $S$. As a cycle $A_{g}$ we may use the cycle $\gamma$ represented by the waist curve of this cylinder. By construction it is independent from the previous ones, and it does not intersect them. To complete the construction of the basis we have to choose a cycle $B_{g}$ dual to $A_{g}$. Consider the following curve representing $B_{g}$ :

- On any cylinder it follows the direction transversal to $\gamma$.

- On the surface $S_{i}^{\prime}$ the curve starts at the boundary component $\gamma_{i}^{\prime \prime}$, then follows in the clockwise direction an arc in a small neighborhood of $z_{i}$, and finally arrives at the boundary component $\gamma_{i}^{\prime}$ in direction transversal to $\gamma$.

The canonical basis of cycles of $S$ is constructed. By construction of this basis the index of any basic curve $A_{i}, B_{i}, i=1, \ldots, g-1$ in the flat structure $S$ does not depend on the way in which we applied the prescribed combination of figure eight constructions. Thus we have

$\sum_{k=1}^{g-1}\left(\operatorname{ind}\left(A_{k}\right)+1\right)\left(\operatorname{ind}\left(B_{k}\right)+1\right)=\sum_{i=1}^{p} \sum_{j_{i}}\left(\operatorname{ind}\left(A_{j_{i}}\right)+1\right)\left(\operatorname{ind}\left(B_{j_{i}}\right)+1\right)=\sum_{i=1}^{p} \phi\left(S_{i}\right)$

Recall that the cycle $A_{g}$ is represented by the waist curve of a cylinder. The tangent vector to the waist curve of the cylinder is constant in the flat structure $\omega$. Thus $\operatorname{ind}\left(A_{g}\right)=0$, which implies that

$$
\begin{aligned}
& \phi(S)=\sum_{k=1}^{g}\left(\operatorname{ind}\left(A_{k}\right)+1\right)\left(\operatorname{ind}\left(B_{k}\right)+1\right)= \\
&=1 \cdot\left(\operatorname{ind}\left(B_{g}\right)+1\right)+\sum_{k=1}^{g-1}\left(\operatorname{ind}\left(A_{k}\right)+1\right)\left(\operatorname{ind}\left(B_{k}\right)+1\right)= \\
&=\operatorname{ind}\left(B_{g}\right)+1+\sum_{i=1}^{p} \phi\left(S_{i}\right)(\bmod 2)
\end{aligned}
$$

It remains to compute the index $\operatorname{ind}\left(B_{g}\right)$. By the construction of $B_{g}$, we may assume that the tangent vector to the corresponding curve does not turn in the flat structure $\omega$ while it crosses the cylinders. It makes a turn by the angle $2 \pi\left(a_{i}^{\prime}+1\right)$ while it follows a small arc joining two holes of a "figure eight" at the point $z_{i}=w_{i}$. This shows that $\operatorname{ind}\left(B_{g}\right)=\sum\left(a_{i}^{\prime}+1\right)(\bmod 2)$.

Lemma 14.3. Suppose $\alpha$ is even so $S$ has a spin structure. If there is some $S_{k}^{\prime}$ to which we apply the parallelogram construction and at least one of the points $z_{k}, w_{k}$ is a zero (marked point) of even order, then all $\alpha_{i}^{\prime}$ are even, and the parity of the spin structure of the flat surface $S$ is equal to the sum of the parities of the spin 
structures of the components

$$
\phi(S)=\sum_{i=1}^{p} \phi\left(S_{i}\right)
$$

Proof. We use the canonical basis on $S$ similar to the one used in the Lemma above. To construct the first $g-1$ pairs of curves we again use the curves living on the component surfaces $S_{i}^{\prime}$. In addition to previous assumptions we require that when we apply creating a pair of holes construction to some $S_{i}$, the basic curves do not approach the transversal $\rho_{i}$ chosen to join $z_{i}$ to $w_{i}$. We extend the description of the curve representing the cycle $B_{g}$ to the surfaces to which we apply the creating a pair of holes construction as follows: on such surfaces $S_{i}^{\prime}$ the curve starts at the boundary component $\gamma_{i}^{\prime \prime}$ in a direction transversal to $\gamma$ and arrives at the boundary component $\gamma_{i}^{\prime}$ in direction transversal to $\gamma$. We choose this curve in such way that it does not intersect any basic curves on $S_{i}^{\prime}$.

It follows from Lemma 14.1 that all the zeroes are of type III; we do not have any glued in cylinders: all the gluings are direct. As a curve representing the cycle $A_{g}$ we now use a small smooth deformation $\tilde{\gamma} \subset S_{k}^{\prime}$ of the boundary curve $\gamma_{i}^{\prime}$, such that $\tilde{\gamma}$ does not pass through any zeroes of $\omega_{i}^{\prime}$. We get $\operatorname{ind}\left(A_{g}\right)=\operatorname{ind}(\tilde{\gamma})=\left(b_{i}^{\prime}+1\right)$. Since the number $b_{i}^{\prime}$ is even, we see that $i n d\left(A_{g}\right)+1=0(\bmod 2)$. Thus the impact of the last pair of cycles to the sum

$$
\phi(S)=\sum_{i=1}^{g}\left(\operatorname{ind}\left(A_{i}\right)+1\right)\left(\operatorname{ind}\left(B_{i}\right)+1\right)
$$

equals to zero, and

$$
\begin{aligned}
\phi(S)=\sum_{i=1}^{g-1}\left(\operatorname{ind}\left(A_{i}\right)+1\right)\left(\operatorname{ind}\left(B_{i}\right)+1\right)= \\
=\sum_{i=1}^{p} \sum_{j_{i}}\left(\operatorname{ind}\left(A_{j_{i}}\right)+1\right)\left(\operatorname{ind}\left(B_{j_{i}}\right)+1\right)=\sum_{i=1}^{p} \phi\left(S_{i}\right)
\end{aligned}
$$

Lemma 14.3 is proved.

Note that there is a discrete freedom left in the figure eight or in the creating a pair of holes construction. When we perform a figure eight construction at a zero of order $a=a^{\prime}+a^{\prime \prime}$ with fixed $a^{\prime}, a^{\prime \prime}$ there are actually $a+1$ ways to perform a figure eight construction, (see Section 12.1). Similarly, when we perform a parallelogram construction at a pair of zeroes of orders $b_{i}^{\prime}, b_{i}^{\prime \prime}$ we, actually obtain $\left(b_{i}^{\prime}+1\right) \cdot\left(b_{i}^{\prime \prime}+1\right)$ different surfaces. Thus, even when we fix all the elements of the collection

$$
\left(S^{\prime}, J, a_{j}^{\prime}, a_{j}^{\prime \prime}, b_{k}^{\prime}, b_{k}^{\prime \prime}\right)
$$

we usually get numerous surfaces $S$ defined by such assignment though some freedom may be cancelled by the symmetry. The collection $\alpha$ of degrees of zeros of the resulting flat structure $S$ is, of course, invariant, but a spin structure may vary.

Lemma 14.4. Let $N$ be the total number of surfaces $S \in \mathcal{H}(\alpha)$ obtained by the assignment

$$
\left(S^{\prime}, J, a_{j}^{\prime}, a_{j}^{\prime \prime}, b_{k}^{\prime}, b_{k}^{\prime \prime}, \gamma, \rho, h, t\right) \rightarrow S
$$

with fixed $\left(S^{\prime}, J, a_{j}^{\prime}, a_{j}^{\prime \prime}, b_{k}^{\prime}, b_{k}^{\prime \prime}, \gamma, \rho, h, t\right)$. Suppose $\alpha$ is even so every such $S$ has a spin structure. If there is some $S_{k}^{\prime}$ to which we apply the creating a pair of holes 
construction, and one of the points $z_{k}, w_{k}$ is an odd order zero, then exactly $N / 2$ of the resulting surfaces have even spin structure and $N / 2$ have odd spin structure.

Proof. Note that if one of $b_{k}^{\prime}, b_{k}^{\prime \prime}$ is odd, the other one is odd as well.

Consider a canonical basis of cycles similar to one constructed in Lemma 14.3. The only difference is that now we may have some cylinders glued in. As in Lemma 14.2 we assume that the curve representing the cycle $B_{g}$ stays transversal to the direction of $\gamma$ while passing through the cylinders.

By the choice of this canonical basis of cycles the index of any basic curve representing $A_{i}, B_{i}, i=1, \ldots, g-1$ in the flat structure $S$ does not depend on the way in which we applied the prescribed combination of figure eight and creating a pair of holes constructions. Thus the number

$$
\phi_{0}:=\sum_{i=1}^{g-1}\left(\operatorname{ind}\left(A_{i}\right)+1\right)\left(\operatorname{ind}\left(B_{i}\right)+1\right)
$$

is invariant of the discrete freedom in the assignment

$$
\left(S^{\prime}, J, a_{j}^{\prime}, a_{j}^{\prime \prime}, b_{k}^{\prime}, b_{k}^{\prime \prime}, \gamma, \rho, h, t\right) \rightarrow S
$$

We construct a representative of the cycle $A_{g}$ as in Lemma 14.3 using a small smooth deformation $\tilde{\gamma} \subset S_{k}^{\prime}$ of the curve $\gamma_{k}^{\prime}$. We get $\operatorname{ind}\left(A_{g}\right)=\operatorname{ind}(\tilde{\gamma})=\left(b_{k}^{\prime}+1\right)$. Since the number $b_{k}^{\prime}$ is odd, we see that $i n d(\tilde{\gamma})+1=1(\bmod 2)$. Thus the parity of the spin structure of $S$ computed in the constructed canonical basis is represented as follows:

$$
\phi(S)=\phi_{0}+\left(\operatorname{ind}\left(A_{g}\right)+1\right)\left(\operatorname{ind}\left(B_{g}\right)+1\right)=\phi_{0}+i n d\left(B_{g}\right)+1(\bmod 2)
$$

Let $z_{i} \neq w_{i}$ be the odd zero of $\omega_{i}^{\prime}$ to which we apply creating a pair of holes construction. Since the conical angle at $z_{i}$ is $2\left(b_{i}^{\prime \prime}+1\right) \pi$, there are an even number $r=b_{i}^{\prime \prime}+1$ of distinct points $P_{1}, \ldots, P_{r}$ of the form $z_{i}+\gamma$. We number the points in the natural cyclic order. Fix all the other elements of the construction leaving the only freedom in the choice of the point $P_{j}$ to perform the slit $z_{i} P_{j}$. We get $r=b_{i}^{\prime \prime}+1$ surfaces $S$. We claim that $r / 2$ of them have even parity of the spin structure, while another $r / 2$ have odd parity of the spin structure.

To see this compare the surfaces obtained from the slits along $z_{i} P_{j}$ and along $z_{i} P_{j+1}$. The two surfaces $S$ share the same collection of curves representing the cycles $A_{i}, B_{i}, i=1, \ldots, g-1$, and $A_{g}$. The curves representing the cycle $B_{g}$ differ only near the point $z_{i}$ : the tangent vector to one of the curves makes an extra turn by the angle $2 \pi$. Thus by equation (33) these two flat surfaces have opposite parities of the spin structures.

14.2. Decomposition of Surfaces from Hyperelliptic Components. In this section we consider possible decompositions of flat surfaces $S$ from hyperelliptic components corresponding to possible configurations of homologous saddle connections joining a zero to itself. In the next section we compute the constants in the quadratic asymptotics for the number of configurations of each type.

We start with the stratum $\mathcal{H}_{1}^{\text {hyp }}(2 g-2)$.

Lemma 14.5. Surfaces $S$ in the hyperelliptic component $\mathcal{H}_{1}^{2 g-2}(\alpha), g \geq 3$ are obtained by an assignment

$$
\left(S^{\prime}, J, a_{j}^{\prime}, a_{j}^{\prime \prime}, b_{k}^{\prime}, b_{k}^{\prime \prime}, \gamma, \rho, h, t\right) \rightarrow S
$$

of one of the following three types: 
i) $(\overline{g-2}, \overline{g-2}) \rightarrow$

The multiplicity is 1 ; the flat surface $S^{\prime}$ belongs to the hyperelliptic component $\mathcal{H}_{1}^{\text {hyp }}(g-2, g-2)$; we apply the creating a pair of holes construction gluing the boundaries directly; the points $z+\gamma, w+\gamma$ in the creating a pair of holes construction are chosen to be symmetric with respect to the hyperelliptic involution on $S^{\prime}$. For fixed data there precisely $g-1$ surfaces $S$ that can be built.

ii) $\overline{((g-2)+(g-2)}) \Rightarrow$

The multiplicity is 1 ; the flat surface $S^{\prime}$ belongs to the hyperelliptic component $\mathcal{H}_{1}^{\text {hyp }}(2 g-4)$; we apply the figure eight construction gluing in a cylinder; $a^{\prime}=a^{\prime \prime}=g-2$. There are $2 g-3$ surfaces $S$ that can be built.

iii) $\left(\overline{\left(g_{1}-1\right)+\left(g_{1}-1\right)}\right) \rightarrow\left(\overline{g_{2}-1}, \overline{g_{2}-1}\right) \rightarrow$

The multiplicity is 2 ; the flat surfaces belong to the hyperelliptic components $S_{1}^{\prime} \in \mathcal{H}^{\text {hyp }}\left(2 g_{1}-2\right), S_{2}^{\prime} \in \mathcal{H}^{\text {hyp }}\left(g_{2}-1, g_{2}-1\right)$, where $g_{1}+g_{2}=g-1$. We apply the figure eight construction with $a_{1}^{\prime}=a_{1}^{\prime \prime}=g_{1}-1$ to the first surface and the creating a pair of holes construction with $b_{2}^{\prime}=b_{2}^{\prime \prime}=g_{2}-1$ to the second one gluing both pairs of components directly. The points $z_{2}+\gamma$, $w_{2}+\gamma \in S_{2}^{\prime}$ are chosen to be symmetric with respect to the hyperelliptic involution on $S_{2}^{\prime}$. There are $g_{2}\left(2 g_{1}-1\right)$ surfaces that can be built.

The surfaces can be related by a stratum interchange or by a $\gamma \rightarrow-\gamma$ symmetry.

The proof is analogous to the proof of Lemma 10.3

Decompositions of the flat surfaces from the hyperelliptic connected component $\mathcal{H}_{1}^{\text {hyp }}(g-1, g-1)$ are described by the following Lemma.

Lemma 14.6. Surfaces $S$ in the hyperelliptic component $\mathcal{H}_{1}^{\text {hyp }}(g-1, g-1)$ are obtained by an assignment

$$
\left(S^{\prime}, J, a_{j}^{\prime}, a_{j}^{\prime \prime}, b_{k}^{\prime}, b_{k}^{\prime \prime}, \gamma, \rho, h, t\right) \rightarrow S
$$

of one of the following two types.

i) $(\overline{g-2}, \overline{g-2}) \Rightarrow$

The multiplicity is $1 ; S^{\prime} \in \mathcal{H}_{1}^{\text {hyp }}(g-2, g-2)$. We apply the parallelogram construction gluing in a cylinder; $b_{1}^{\prime}=b_{1}^{\prime \prime}=g-2$. The points $z+\gamma, w+\gamma$ in the parallelogram construction are chosen to be symmetric with respect to the hyperelliptic involution on $S^{\prime}$. For fixed data there are precisely $g-1$ surfaces $S$ that can be built.

ii) $\left.\overline{\left(g_{1}-1\right)}, \overline{\left(g_{1}-1\right)}\right) \rightarrow\left(\overline{g_{2}-1}, \overline{g_{2}-1}\right) \rightarrow$

The multiplicity is $2 ; S_{i}^{\prime} \in \mathcal{H}^{\text {hyp }}\left(g_{i}-1, g_{i}-1\right)$. where $g_{1}+g_{2}=g-1$. We apply two creating a pair of holes constructions gluing the components directly. The points $z_{i}+\gamma, w_{i}+\gamma$ in the creating a pair of holes constructions are chosen to be symmetric with respect to the hyperelliptic involution on each $S_{i}^{\prime}, i=1,2$. For the fixed data the number of surfaces $S$ that can be built is $g_{1} \cdot g_{2}$ for $g_{1} \neq g_{2}$ and $g_{1} \cdot g_{2} / 2$ for $g_{1}=g_{2}$.

Surfaces can be related by a stratum interchange or by a $\gamma \rightarrow-\gamma$ symmetry.

The proof is analogous to the proof of Lemma 10.3

14.3. Constants for the Hyperelliptic Connected Components. The computation of the constants is based essentially on (32). However, since the flat 
surfaces in the hyperelliptic components have extra symmetry we have to make necessary adjustments.

14.3.1. Hyperelliptic Component $\mathcal{H}_{1}^{\text {hyp }}(2 g-2)$. The admissible assignments for this component are described in Lemma 14.5. We always have the $\gamma \rightarrow-\gamma$ symmetry for all three assignments. We never have any stratum interchange symmetry.

The only modification which we need to make in equation (32) is as follows. Applying the parallelogram constructions in this case we need to choose the points $z_{i}+\gamma, w_{i}+\gamma$ to be symmetric with respect to hyperelliptic involution on each $S_{i}$ (see Lemma 14.5). Note that for this component we always have $b_{k}^{\prime}=b_{k}^{\prime \prime}$ in any assignment containing a creating a pair of holes construction. Thus we have to replace each factor $\left(b_{k}^{\prime}+1\right)\left(b_{k}^{\prime \prime}+1\right)$ by the corresponding factor $\left(b_{k}^{\prime}+1\right)$. We get the following

Formula 14.1. For almost all flat surfaces in a hyperelliptic connected component $\mathcal{H}_{1}^{\text {hyp }}(2 g-2), g \geq 3$, there are only three possible types of configurations of saddle connections joining zero to itself. The constants in the quadratic asymptotics for the number of saddle connections of each type are presented by the following formulae:

i) Assignment $(\overline{g-2}, \overline{g-2}) \rightarrow$

$$
c=\frac{g-1}{2} \cdot \frac{\operatorname{Vol}\left(\mathcal{H}_{1}^{h y p}(g-2, g-2)\right)}{\operatorname{Vol}\left(\mathcal{H}_{1}^{\text {hyp }}(2 g-2)\right)}
$$

ii) Assignment $(\overline{(g-2)+(g-2)}) \Rightarrow$

$$
c=\frac{1}{2} \cdot \frac{2 g-3}{2 g-2} \cdot \frac{\operatorname{Vol}\left(\mathcal{H}_{1}^{h y p}(2 g-4)\right)}{\operatorname{Vol}\left(\mathcal{H}_{1}^{h y p}(2 g-2)\right)}
$$

iii) Assignment $\left(\overline{\left(g_{1}-1\right)+\left(g_{1}-1\right)}\right) \rightarrow\left(\overline{g_{2}-1}, \overline{g_{2}-1}\right) \rightarrow$

$c=\frac{\left(2 g_{1}-1\right) g_{2}}{4} \cdot \frac{\left(2 g_{1}-1\right) !\left(2 g_{2}\right) !}{(2 g-2) !} \cdot \frac{\operatorname{Vol}\left(\mathcal{H}_{1}^{h y p}\left(2 g_{1}-2\right)\right) \operatorname{Vol}\left(\mathcal{H}_{1}^{h y p}\left(g_{2}-1, g_{2}-1\right)\right)}{\operatorname{Vol}\left(\mathcal{H}_{1}^{h y p}(2 g-2)\right)}$

where $g_{1}+g_{2}=g-1$.

Example 14.7. Stratum $\mathcal{H ( 2 ) . ~ T h i s ~ s t r a t u m ~ i s ~ c o n n e c t e d ; ~ i t ~ c o i n c i d e s ~ w i t h ~ i t s ~}$ hyperelliptic component. Flat surfaces from this stratum have a single zero of order 2 . It is easy to see that in genus $g=2$ only multiplicity one is realizable.

$\rightarrow(\overline{\mathbf{0}}, \overline{\mathbf{0}}) \rightarrow$

In this case is a single saddle connection returning with angle $3 \pi$ so that there is no cylinder. After collapsing this saddle connection we get a torus with two marked points to which we apply the parallelogram construction, gluing the circles directly. Here we have

$$
c=\frac{2-1}{2} \cdot \frac{\operatorname{Vol}\left(\mathcal{H}_{1}(0)\right)}{\operatorname{Vol}\left(\mathcal{H}_{1}(2)\right)}=\frac{10}{3} \cdot \frac{1}{\zeta(2)} \approx 2.026
$$

$\Rightarrow(\overline{\mathbf{0}}) \Rightarrow$

The other possibility is that the saddle connection returns at angle $\pi$. Then there is a whole cylinder filled with homologous closed geodesics. In particular, there is another saddle connection returning at the angle $\pi$ homologous to the initial one. 
After collapsing the cylinder we get a torus with a single marked point. We apply the figure eight construction.

$$
c=\frac{1}{4} \cdot \frac{\operatorname{Vol}\left(\mathcal{H}_{1}(0)\right)}{\operatorname{Vol}\left(\mathcal{H}_{1}(2)\right)}=\frac{5}{3} \cdot \frac{1}{\zeta(2)} \approx 1.013
$$

Example 14.8. Component $\mathcal{H}^{\text {hyp }}(4)$. In genus $g=3$ all three possible assignments (see Lemma 14.5) are already admissible.

$\rightarrow(\overline{1}, \overline{1}) \rightarrow$

The first represents the multiplicity 1 case of a saddle connection returning at angle $5 \pi$.

$$
c=\frac{3-1}{2} \cdot \frac{\operatorname{Vol}(\mathcal{H}(1,1))}{\operatorname{Vol}\left(\mathcal{H}^{\text {hyp }}(4)\right)}=\frac{224}{27} \cdot \frac{1}{\zeta(2)} \approx 5.044
$$

$\Rightarrow(\overline{1+1}) \Rightarrow$

If there is a cylinder, by symmetry, the spacing of the angles between the boundaries of the cylinders is $4 \pi$. Then after degeneration, we get a single zero of order 2 on a surface of genus 2 . We apply the figure eight construction with $a_{1}^{\prime}=a_{1}^{\prime \prime}=1$, and glue in a cylinder. We get

$$
c=\frac{1}{2} \cdot \frac{3}{4} \cdot \frac{\operatorname{Vol}(\mathcal{H}(2))}{\operatorname{Vol}\left(\mathcal{H}^{\text {hyp }}(4)\right)}=\frac{7}{2} \cdot \frac{1}{\zeta(2)} \approx 2.128
$$

$\rightarrow(\overline{\mathbf{0}+\mathbf{0}}) \rightarrow(\overline{\mathbf{0}}, \overline{\mathbf{0}}) \rightarrow$

In the multiplicity $p=2$ case we have two homologous curves, both returning to $z_{0}$ at angles $3 \pi$. After degeneration we get two tori, one with two marked points, the other with one. We perform the figure eight construction on one torus and the creating a pair of holes construction on the other, gluing the boundaries directly to each other.

$$
c=\frac{(2 \cdot 1-1) \cdot 1}{4} \cdot \frac{(2 \cdot 1-1) !(2 \cdot 1) !}{(6-1) !} \cdot \frac{\operatorname{Vol}(\mathcal{H}(0))^{2}}{\operatorname{Vol}\left(\mathcal{H}^{\text {hyp }}(4)\right)}=\frac{70}{27} \cdot \frac{1}{\zeta(2)} \approx 1.576
$$

14.3.2. Hyperelliptic Component $\mathcal{H}^{\text {hyp }}(g-1, g-1)$. The admissible assignments for this component are described by Lemma 14.6] We always have the $\gamma \rightarrow-\gamma$ symmetry for both assignments. We have the stratum interchange symmetry in the second assignment if and only if $g_{1}=g_{2}$.

The only modification which we need to make in the equation (32) is as follows. Applying the parallelogram constructions in this case we need to choose the points $z_{i}+\gamma, w_{i}+\gamma$ to be symmetric with respect to hyperelliptic involution on each $\left(S_{i}, \omega_{i}\right)$ (see Lemma 14.6). This means that we have to replace each factor $\left(b_{k}^{\prime}+1\right)\left(b_{k}^{\prime \prime}+1\right)$ by the corresponding factor $\left(b_{k}^{\prime}+1\right)$. Thus we get the following

Formula 14.2. For almost all flat surfaces in the hyperelliptic connected component $\mathcal{H}^{\text {hyp }}(g-1, g-1), g \geq 3$, there are only two possible types of configurations of saddle connections joining any of two zeroes to itself. The constants in the quadratic asymptotics for the number of saddle connections of each type are presented by the following formulae:

i) Assignment $(\overline{g-2}, \overline{g-2}) \Rightarrow$

$$
c=\frac{(g-1)}{(2 g-1)} \cdot \frac{\operatorname{Vol}\left(\mathcal{H}_{1}^{\text {hyp }}(g-2, g-2)\right)}{\operatorname{Vol}\left(\mathcal{H}_{1}^{\text {hyp }}(g-1, g-1)\right)}
$$




$$
\begin{aligned}
& \text { ii) Assignment }\left(\overline{\left(g_{1}-1\right)}, \overline{\left(g_{1}-1\right)}\right) \rightarrow\left(\overline{g_{2}-1}, \overline{g_{2}-1}\right) \rightarrow \\
& c=\frac{g_{1} g_{2}}{2|\Gamma|} \cdot \frac{\left(2 g_{1}\right) !\left(2 g_{2}\right) !}{(2 g-1) !} \cdot \frac{\operatorname{Vol}\left(\mathcal{H}_{1}^{\text {hyp }}\left(g_{1}-1, g_{1}-1\right)\right) \operatorname{Vol}\left(\mathcal{H}_{1}^{\text {hyp }}\left(g_{2}-1, g_{2}-1\right)\right)}{\operatorname{Vol}\left(\mathcal{H}_{1}^{\text {hyp }}(g-1, g-1)\right)} .
\end{aligned}
$$

where $g_{1}+g_{2}=g-1$, and

$$
|\Gamma|= \begin{cases}1 & \text { when } g_{1} \neq g_{2} \\ 2 & \text { when } g_{1}=g_{2}\end{cases}
$$

Example 14.9. Stratum $\mathcal{H}(\mathbf{1}, \mathbf{1})$. In genus $g=2$ the stratum $\mathcal{H}(1,1)$ is the principal stratum. It is connected, and it coincides with the hyperelliptic component $\mathcal{H}(1,1)=\mathcal{H}^{\text {hyp }}(1,1)$.

Since $g=g_{1}+g_{2}+1$, and $g_{i} \geq 1$, we see that multiplicity two does not occur in genus $g=2$. The value

$$
c=\frac{1}{3} \cdot \frac{\operatorname{Vol}\left(\mathcal{H}_{1}^{\text {hyp }}(0)\right)}{\operatorname{Vol}\left(\mathcal{H}_{1}^{\text {hyp }}(1,1)\right)}=\frac{5}{2}
$$

of the constant in multiplicity one was computed in section 13.5

Example 14.10. Component $\mathcal{H}^{\text {hyp }}(\mathbf{2 , 2})$.

$\Rightarrow(\overline{\mathbf{1}}, \overline{\mathbf{1}}) \Rightarrow$

In the multiplicity 1 case there is a cylinder returning to the other zero. Here we get

$$
c=\frac{2}{5} \cdot \frac{\operatorname{Vol}\left(\mathcal{H}_{1}(1,1)\right)}{\operatorname{Vol}\left(\mathcal{H}_{1}^{\text {hyp }}(2,2)\right)}=\frac{14}{3} \cdot \frac{1}{\zeta(2)} \approx 2.837
$$

$\rightarrow(\overline{\mathbf{0}}, \overline{\mathbf{0}}) \rightarrow(\overline{\mathbf{0}}, \overline{\mathbf{0}}) \rightarrow$

In the multiplicity 2 case we have homologous curves, neither of which bounds a cylinder, one returning to each zero. The degenerating surfaces are tori each with 2 marked points on each. We perform the parallelogram construction, gluing the circles pairwise directly. Since $g_{1}=g_{2}$ we get not only the $\gamma \rightarrow-\gamma$ but also the stratum interchange, $|\Gamma|=2$.

$$
c=\frac{1 \cdot 1}{2 \cdot 2} \cdot \frac{(2 \cdot 1) !(2 \cdot 1) !}{(2 \cdot 3-1) !} \cdot \frac{\operatorname{Vol}\left(\mathcal{H}_{1}(0)\right)^{2}}{\operatorname{Vol}\left(\mathcal{H}_{1}^{\text {hyp }}(2,2)\right)}=\frac{35}{24} \cdot \frac{1}{\zeta(2)} \approx 0.8866
$$

14.4. Connected Component $\mathcal{H}^{\text {nonhyp }}(g-1, g-1)$; Even Genus $g$. When the genus $g \geq 4$ is even, the stratum $\mathcal{H}(g-1, g-1)$ has two connected components: the hyperelliptic one, $\mathcal{H}^{h y p}(g-1, g-1)$, which we considered in the previous section, and the nonhyperelliptic connected component $\mathcal{H}^{\text {nonhyp }}(g-1, g-1)$. The description of admissible constructions for the connected component $\mathcal{H}^{\text {nonhyp }}(g-1, g-1)$ for even $g \geq 4$ is completely analogous to the case of connected strata. However, in the following two assignments

$$
(\overline{g-2}, \overline{g-2}) \Rightarrow
$$

and

$$
\left(\overline{\left(g_{1}-1\right)}, \overline{\left(g_{1}-1\right)}\right) \rightarrow\left(\overline{g_{2}-1}, \overline{g_{2}-1}\right) \rightarrow
$$

we have interference with the hyperelliptic components. Thus in these two cases we have to modify the general formula 13.1 by subtracting the correction term corresponding to the constructions leading to hyperelliptic flat surfaces, see formula 14.2 . 
Formula 14.3. For almost all flat surfaces in a nonhyperelliptic connected component $\mathcal{H}^{\text {nonhyp }}(g-1, g-1)$ for even $g \geq 4$, the constants in the quadratic asymptotics for the number of saddle connections of each of the following two types are presented by the following formulae:

i) Assignment $(\overline{g-2}, \overline{g-2}) \Rightarrow$

$$
\begin{aligned}
& c=\frac{(g-1)^{2}}{(2 g-1)} \cdot \frac{\operatorname{Vol}\left(\mathcal{H}_{1}(g-2, g-2)\right)}{\operatorname{Vol}\left(\mathcal{H}_{1}^{\text {nonhyp }}(g-1, g-1)\right)}-\frac{(g-1)}{(2 g-1)} \cdot \frac{\operatorname{Vol}\left(\mathcal{H}_{1}^{\text {hyp }}(g-2, g-2)\right)}{\operatorname{Vol}\left(\mathcal{H}_{1}^{\text {nonhyp }}(g-1, g-1)\right)} \\
& \text { ii) Assignment }\left(\overline{\left(g_{1}-1\right)}, \overline{\left(g_{1}-1\right)}\right) \rightarrow\left(\overline{g_{2}-1}, \overline{g_{2}-1}\right) \rightarrow \\
& c=\frac{\left(g_{1}\right)^{2}\left(g_{2}\right)^{2}}{2|\Gamma|} \cdot \frac{\left(2 g_{1}\right) !\left(2 g_{2}\right) !}{(2 g-1) !} \cdot \frac{\operatorname{Vol}\left(\mathcal{H}_{1}\left(g_{1}-1, g_{1}-1\right)\right) \operatorname{Vol}\left(\mathcal{H}_{1}\left(g_{2}-1, g_{2}-1\right)\right)}{\operatorname{Vol}\left(\mathcal{H}_{1}^{\text {nonhyp }}(g-1, g-1)\right)}- \\
& -\frac{g_{1} g_{2}}{2|\Gamma|} \cdot \frac{\left(2 g_{1}\right) !\left(2 g_{2}\right) !}{(2 g-1) !} \cdot \frac{\operatorname{Vol}\left(\mathcal{H}_{1}^{\text {hyp }}\left(g_{1}-1, g_{1}-1\right)\right) \operatorname{Vol}\left(\mathcal{H}_{1}^{\text {hyp }}\left(g_{2}-1, g_{2}-1\right)\right)}{\operatorname{Vol}\left(\mathcal{H}_{1}^{\text {nonhyp }}(g-1, g-1)\right)}
\end{aligned}
$$

where $g_{1}+g_{2}=g-1$, and

$$
|\Gamma|= \begin{cases}1 & \text { when } g_{1} \neq g_{2} \\ 2 & \text { when } g_{1}=g_{2}\end{cases}
$$

iii) The constants for all other assignments are given by equation (32), with $\alpha=(g-1, g-1)$ where $\operatorname{Vol}(\mathcal{H}(g-1, g-1))$ in the denominator of the rightmost fraction should be replaced by $\operatorname{Vol}\left(\mathcal{H}_{1}^{\text {nonhyp }}(g-1, g-1)\right)$.

As an example of this formula we present the list of possible generic degenerations of a flat surface $S \in \mathcal{H}^{\text {nonhyp }}(3,3)$ in Appendix B

Remark 14.11. Note, that one can represent the constant corresponding to the two exceptional assignments in the formula above as

$$
c=c_{\text {regular }}-c_{\text {hyp }} \cdot \frac{\operatorname{Vol}\left(\mathcal{H}^{\text {hyp }}(g-1, g-1)\right)}{\operatorname{Vol}\left(\mathcal{H}^{\text {nonhyp }}(g-1, g-1)\right)} .
$$

Here $c_{\text {hyp }}$ in the correctional term is the constant for the corresponding assignment for the hyperelliptic component $\mathcal{H}^{\text {hyp }}(g-1, g-1)$, see Formula 14.2

14.5. Nonhyperelliptic Components with a Spin Structure. Let now $\alpha$ be even and let $S$ belong to one of the nonhyperelliptic components $\mathcal{H}_{1}^{\text {even }}(\alpha)$ or $\mathcal{H}^{\text {odd }}(\alpha)$. The admissible assignments for such flat surfaces are described by the general Lemmas 14.2, 14.3, 14.4

When $\alpha$ is one of the two partitions $(2 g-2),(g-1, g-1)$ there are special assignments where there might be interference with hyperelliptic components, see Lemmas 14.5 and 14.6.

In the formula below we use a function $\delta\left(\alpha^{\prime}, \phi^{\prime}\right)$ which is equal to 1 when the stratum $\mathcal{H}\left(\alpha^{\prime}\right)$ contains a hyperelliptic component, and when, moreover, this hyperelliptic component has parity $\phi^{\prime}$ of the spin structure. This function is equal to zero otherwise (see (25) for the explicit definition). By convention we let the volume of several "missing" components in small genera and the volume of nonexisting hyperelliptic components be equal to zero (see (26) for the complete list). 
Formula 14.4. Let all the integers in $\alpha$ be even, and let $\alpha$ be different from any of the partitions $(2 g-2),(g-1, g-1)$.

For almost all flat surfaces in the connected component $\mathcal{H}^{\phi}(\alpha)$ the constants in the quadratic asymptotics for the number of saddle connections joining some zero to itself are presented by the following formulae:

- If the assignment contains only figure eight constructions

$$
\begin{gathered}
c=\frac{1}{\left|\Gamma_{-}\right| \cdot|\Gamma|} \cdot \prod_{m \in \alpha}\left(\frac{o(m) !}{\prod_{i=1}^{p} o_{i}(m) !}\right) \cdot \prod_{\substack{1 \leq i \leq p \\
a_{i} \in \alpha}} o_{i}\left(a_{i}\right) \cdot \prod_{1 \leq i \leq p}\left(a_{i}+1\right) \\
\cdot \frac{1}{2^{p-1}} \cdot \frac{\prod_{i=1}^{p}\left(\frac{d_{i}}{2}-1\right) !}{\left(\frac{d}{2}-2\right) !} \cdot \frac{1}{\operatorname{Vol}\left(\mathcal{H}_{1}^{\phi}(\alpha)\right)} \cdot \\
\sum_{\substack{\phi_{1}^{\prime}, \ldots, \phi_{p}^{\prime} \in\{\text { even }, \text { odd }\} \\
\left(\phi_{1}^{\prime}+\cdots+\phi_{p}^{\prime}\right)+\\
+\left(a_{1}^{\prime}+\dot{+} a_{p}^{\prime}\right)+\\
+p+1 \equiv \phi(\bmod 2)}} \prod_{\substack{p \\
+}}\left(\operatorname{Vol}\left(\mathcal{H}_{1}^{\phi_{i}}\left(\alpha_{i}^{\prime}\right)\right)+\delta\left(\alpha_{i}^{\prime}, \phi_{i}^{\prime}\right) \operatorname{Vol}\left(\mathcal{H}_{1}^{h y p}\left(\alpha_{i}^{\prime}\right)\right)\right) \\
\end{gathered}
$$

- If the assignment contains at least one parallelogram construction with even $b_{i}^{\prime}$

$$
\begin{gathered}
c=\frac{1}{\left|\Gamma_{-}\right|} \cdot \frac{1}{|\Gamma|} \cdot \prod_{m \in \alpha}\left(\frac{o(m) !}{\prod_{i=1}^{p} o_{i}(m) !}\right) \cdot \\
\cdot \prod_{\substack{1 \leq i \leq p \\
z_{i}=w_{i} \\
a_{i} \in \alpha}} o_{i}\left(a_{i}\right) \cdot \prod_{\substack{1 \leq k \leq p \\
z_{k} \neq w_{k} \\
b_{k}^{\prime} \neq b_{k}^{\prime \prime} \\
b_{k}^{\prime} \in \alpha}} o_{k}\left(b_{k}^{\prime}\right) \cdot \prod_{\substack{1 \leq k \leq p \\
z_{k} \neq w_{k}^{\prime} \\
b_{k}^{\prime} \neq b_{k}^{\prime \prime} \\
b_{k}^{\prime \prime} \in \alpha}} o_{k}\left(b_{k}^{\prime \prime}\right) \cdot \prod_{\substack{1 \leq k \leq p \\
z_{k} \neq w_{k} \\
b_{k}^{\prime}=b_{k}^{\prime \prime} \in \alpha}} o_{k}\left(b_{k}^{\prime}\right)\left(o_{k}\left(b_{k}^{\prime}\right)-1\right) \cdot \\
\cdot \prod_{\substack{1 \leq i \leq p \\
z_{i}=w_{i}}}\left(a_{i}+1\right) \cdot \prod_{\substack{1 \leq k \leq p \\
z_{k} \neq w_{k}}}\left(b_{k}^{\prime}+1\right)\left(b_{k}^{\prime \prime}+1\right) \cdot \frac{1}{2^{p-1}} \cdot \frac{\prod_{i=1}^{p}\left(\frac{d_{i}}{2}-1\right) !}{\left(\frac{d}{2}-2\right) !} \cdot \\
\frac{1}{\operatorname{Vol}\left(\mathcal{H}_{1}^{\phi}(\alpha)\right)} \cdot \sum_{\substack{\phi_{1}^{\prime}, \ldots, \phi_{p}^{\prime} \in\{\text { even, odd }\} \\
\left(\phi_{1}^{\prime}+\cdots+\phi_{p}^{\prime}\right) \equiv \phi(\bmod 2)}}^{p}\left(\operatorname{Vol}\left(\mathcal{H}_{1}^{\phi_{i}}\left(\alpha_{i}^{\prime}\right)\right)+\delta\left(\alpha_{i}^{\prime}, \phi_{i}^{\prime}\right) \operatorname{Vol}\left(\mathcal{H}_{1}^{h y p}\left(\alpha_{i}^{\prime}\right)\right)\right)
\end{gathered}
$$


- If the assignment contains at least one parallelogram construction with odd $b_{i}^{\prime}$

$$
\begin{gathered}
c=\frac{1}{|\Gamma-|} \cdot \frac{1}{|\Gamma|} \cdot \prod_{m \in \alpha}\left(\frac{o(m) !}{\prod_{i=1}^{p} o_{i}(m) !}\right) \cdot \\
\cdot \prod_{\substack{1 \leq i \leq p \\
z_{i}=w_{i} \\
a_{i} \in \alpha}} o_{i}\left(a_{i}\right) \cdot \prod_{\substack{1 \leq k \leq p \\
z_{k} \neq w_{k} \\
b_{k}^{\prime} \neq b_{k}^{\prime \prime} \\
b_{k}^{\prime} \in \alpha}} o_{k}\left(b_{k}^{\prime}\right) \cdot \prod_{\substack{1 \leq k \leq p \\
z_{k} \neq w_{k} \\
b_{k}^{\prime} \neq b_{k}^{\prime \prime} \\
b_{k}^{\prime \prime} \in \alpha}} o_{k}\left(b_{k}^{\prime \prime}\right) \cdot \prod_{\substack{1 \leq k \leq p \\
z_{k} \neq w_{k} \\
b_{k}^{\prime}=b_{k}^{\prime \prime} \in \alpha}} o_{k}\left(b_{k}^{\prime}\right)\left(o_{k}\left(b_{k}^{\prime}\right)-1\right) \cdot \\
\cdot \prod_{\substack{1 \leq i \leq p \\
z_{i}=w_{i}}}\left(a_{i}+1\right) \cdot \prod_{\substack{1 \leq k \leq p \\
z_{k} \neq w_{k}}}\left(b_{k}^{\prime}+1\right)\left(b_{k}^{\prime \prime}+1\right) \cdot \\
\qquad \frac{1}{2} \cdot \frac{1}{2^{p-1}} \cdot \frac{\prod_{i=1}^{p}\left(\frac{d_{i}}{2}-1\right) !}{\left(\frac{d}{2}-2\right) !} \cdot \frac{\prod_{i=1}^{p} \operatorname{Vol}\left(\mathcal{H}_{1}\left(\alpha_{i}^{\prime}\right)\right)}{\operatorname{Vol}\left(\mathcal{H}_{1}^{\phi}(\alpha)\right)}
\end{gathered}
$$

Proof. The formula above is obtained by elementary adjustment of formula (32).

First suppose that the assignment under consideration does not involve any parallelogram constructions with odd zeroes. Then by Lemma 14.2 and Lemma 14.3 the spin structure of $S$ is determined by the spin structures of $S_{i}^{\prime}$ and by the parities of $a_{i}^{\prime}$. Thus the combinatorial constant is exactly the same as in equation (32). The numerator of the volume term is the sum over those products of $\operatorname{Vol}\left(\mathcal{H}^{\text {even }}\left(\alpha_{i}\right)\right)$, $\operatorname{Vol}\left(\mathcal{H}^{o d d}\left(\alpha_{j}\right)\right)$, and $\operatorname{Vol}\left(\mathcal{H}^{h y p}\left(\alpha_{i}\right)\right)$ (when appropriate) that satisfy the relevant relation between spin structures of component surfaces and the spin structure of the resulting surface, see Lemma 14.2 and Lemma 14.3 correspondingly. In the denominator of the volume term we have the volume of the component under consideration.

If there is a parallelogram construction with odd $b_{k}^{\prime}$ involved in the assignment, then by Lemma 14.4 we have to multiply $c$ by $1 / 2$ to account for the fact that half of the constructions produce flat surfaces with even spin structure and another half of the constructions produce a flat surface with odd spin structure. Thus we need to divide by 2 the combinatorial factor in equation (32). In this case the numerator of the volume term is same as in (32): it is a product of $\operatorname{Vol}\left(\mathcal{H}_{1}\left(\alpha_{i}\right)\right)$, where we take the total volume of all connected components in the stratum. In the denominator of the volume term we again have the volume of the component under consideration.

We complete this section considering the exceptional strata.

Formula 14.5. Let $\alpha=(2 g-2)$, or let $\alpha=(g-1, g-1)$ where $g$ is odd. Let $g \geq 4$, or let $g=3$ and $\phi=$ odd.

For almost all flat surfaces in the connected component $\mathcal{H}^{\phi}(\alpha)$ the constants in the quadratic asymptotics for the number of saddle connections joining some zero to itself are presented by the following formulae:

- If the assignment is different from any of the assignments listed in Lemmas 14.5, and 14.6. then the constant is the same as in Formula 14.4.

- For any of the assignments listed in one of the Lemmas 14.5. and 14.6 the constant is equal to

$$
c=c_{\text {regular }}-c_{\text {hyp }} \cdot \frac{\operatorname{Vol}\left(\mathcal{H}^{\text {hyp }}(\alpha)\right)}{\operatorname{Vol}\left(\mathcal{H}^{\phi}(\alpha)\right)} .
$$


Here $c_{\text {regular }}$ is given by Formula 14.4 and $c_{\text {hyp }}$ is the constant for the corresponding assignment for the hyperelliptic component $\mathcal{H}^{\text {hyp }}(\alpha)$, see Formulae 14.1 and 14.2 .

Proof. For nonexceptional assignments the proof is exactly the same as for Formula 14.4 For the exceptional assignments, which may lead to the flat surfaces from hyperelliptic components one makes appropriate adjustment, see the proof of Formula 14.3 and Remark 14.11 after this proof.

Example 14.12. Component $\mathcal{H}^{\text {odd }}(\mathbf{4})$. Recall that the stratum $\mathcal{H}(4)$ has only one nonhyperelliptic connected component $\mathcal{H}^{\text {odd }}(4)$. Every flat surface in $\mathcal{H}(4)$ having even parity of the spin structure belongs to $\mathcal{H}^{h y p}(4)$. We have $d=\operatorname{dim}_{\mathbb{R}} \mathcal{H}(4)=12$. Consider possible assignments for the flat surfaces from the component $\mathcal{H}^{\text {odd }}(4)$. We start with assignments of multiplicity one.

$\rightarrow(\overline{2}, \overline{0}) \rightarrow$

We have a single saddle connection that does not bound a cylinder. It returns at angle $3 \pi$. After degeneration we get a surface of genus 2 with a zero of order 2 and with a marked point. We apply the parallelogram construction gluing the circles directly. Since $b_{1}^{\prime}=2$, and $b_{1}^{\prime \prime}=0$ are even, by lemma 14.3 the parity of the spin structure of the resulting surface coincides with the parity of the spin structure of a surface from $\mathcal{H}(2)$, so it is really odd, see (8). Since $b_{1}^{\prime} \neq b_{2}^{\prime \prime}$ we do not have a $\gamma \rightarrow-\gamma$ symmetry. Thus,

$$
c=3 \cdot \frac{(5-1) !}{(6-2) !} \cdot \frac{\operatorname{Vol}(\mathcal{H}(2))}{\operatorname{Vol}\left(\mathcal{H}^{\text {odd }}(4)\right)}=\frac{81}{8} \cdot \frac{1}{\zeta(2)} \approx 6.155
$$

$\rightarrow(\overline{\mathbf{1}}, \overline{\mathbf{1}}) \rightarrow$

Here we again have a single saddle connection that does not bound a cylinder, but now it returns at angle $5 \pi$. After degeneration we get a surface of genus 2 with a pair of simple zeroes. We apply the parallelogram construction gluing the circles directly. Since $b_{1}^{\prime}=1$, and $b_{1}^{\prime \prime}=1$ we get $N=\left(b_{1}^{\prime}+1\right)\left(b_{1}^{\prime \prime}+1\right)=4$ surfaces, but only half of them have odd parity of the spin structure, see lemma 14.4. Now we have a $\gamma \rightarrow-\gamma$ symmetry, $\left|\Gamma_{-}\right|=2$.

$$
c=1 \cdot \frac{(5-1) !}{(6-2) !} \cdot \frac{\operatorname{Vol}(\mathcal{H}(1,1))}{\operatorname{Vol}\left(\mathcal{H}^{\text {odd }}(4)\right)}=3 \cdot \frac{1}{\zeta(2)} \approx 1.824
$$

$\Rightarrow(\overline{0+2}) \Rightarrow$

Suppose now that the saddle connection returns at angle $\pi$. Then it bounds a cylinder filled with homologous closed geodesics. In particular, there is another saddle connection returning at the angle $\pi$ homologous to the initial one. Since the surface is not hyperelliptic, the angle between the saddle connections on the opposite sides of the cylinder is $6 \pi$. After degeneration we get a single zero of order 2 on a surface of genus 2 . We apply the figure eight construction, breaking the zero of order 2 into a zero of order 2 and one of order 0 . This corresponds to spacings of $2 \pi$ and $6 \pi$, or, in our notation to the choice $a_{1}^{\prime}=0, a_{1}^{\prime \prime}=2$. We glue in an intervening cylinder. By lemma 14.2 the parity of the spin structure of the resulting flat surface equals $1+\phi\left(S_{1}^{\prime}\right)+(0+1)=1$ since $\phi\left(S_{1}^{\prime}\right)=1$, see (8). Since $a_{1}^{\prime} \neq a_{1}^{\prime \prime}$ we do not have the $\gamma \rightarrow-\gamma$ symmetry.

$$
c=3 \cdot \frac{(4-1) !}{(6-2) !} \cdot \frac{\operatorname{Vol}(\mathcal{H}(2))}{\operatorname{Vol}\left(\mathcal{H}^{\text {odd }}(4)\right)}=\frac{81}{32} \cdot \frac{1}{\zeta(2)} \approx 1.539
$$


$\Rightarrow(\overline{0+0}) \rightarrow(\overline{0+0}) \Rightarrow$

We now consider multiplicity 2 . We have a cylinder and a homologous curve that does not bound a cylinder. The degenerated surfaces $S_{1}^{\prime}, S_{2}^{\prime}$ are a pair of tori, each with a marked point. We apply the figure eight construction giving a pair of circles on each torus. We glue one pair of circles directly and put an intervening cylinder between the other pair. Though $\alpha_{1}^{\prime}=\alpha_{2}^{\prime}$ we do not have a stratum interchange, $|\Gamma|=1$, but we have the $\gamma \rightarrow-\gamma$ symmetry.

$$
c=\frac{1}{2} \cdot \frac{1}{2^{2-1}} \cdot \frac{(2-1) ! \cdot(2-1) !}{(6-2) !} \cdot \frac{\operatorname{Vol}(\mathcal{H}(0))^{2}}{\operatorname{Vol}\left(\mathcal{H}^{\text {odd }}(4)\right)}=\frac{15}{32} \cdot \frac{1}{\zeta(2)} \approx 0.2850
$$

Example 14.13. Component $\mathcal{H}^{\text {odd }}(\mathbf{2}, \mathbf{2})$.

$\rightarrow(2, \overline{0}, \overline{0}) \rightarrow$

In the multiplicity 1 case with no cylinder, the degenerating surface is genus 2 with a pair of marked points and a zero of order 2 . We perform the creating a pair of holes construction on the marked points, gluing the circles directly. Since $b_{1}^{\prime}=b_{1}^{\prime \prime}=0$ is even, by lemma 14.3 the parity of the spin structure equals $\phi\left(S^{\prime}\right)$, which is odd for $S^{\prime} \in \mathcal{H}(2)$.

$$
c=1 \cdot \frac{(4+2-1) !}{(7-2) !} \cdot \frac{\operatorname{Vol}\left(\mathcal{H}_{1}(2)\right)}{\operatorname{Vol}\left(\mathcal{H}_{1}^{\text {odd }}(2,2)\right)}=6 \cdot \frac{1}{\zeta(2)} \approx 3.648
$$

$\Rightarrow(2, \overline{0+0}) \Rightarrow$

In the case of a cylinder with multiplicity 1 , if the other side returns to the same zero, we get a genus 2 surface with a single marked point and a double zero. We perform the figure eight construction at the marked point, gluing in a cylinder to the two circles. We have $a_{1}^{\prime}=a_{1}^{\prime \prime}=0$. Thus, by lemma 14.2 $\phi(S)=1+\phi\left(S^{\prime}\right)+(0+1)=$ $1+1+1=1 \bmod 2$. Now $\left|\Gamma_{-}\right|=2$.

$$
c=1 \cdot \frac{(4+1-1) !}{(7-2) !} \cdot \frac{\operatorname{Vol}\left(\mathcal{H}_{1}(2)\right)}{\operatorname{Vol}\left(\mathcal{H}_{1}^{\text {odd }}(2,2)\right)}=\frac{6}{5} \cdot \frac{1}{\zeta(2)} \approx 0.7295
$$

$\Rightarrow(\overline{1}, \overline{1}) \Rightarrow$

If the other side of the cylinder returns to the other zero, after degeneration we get 2 simple zeroes. We perform the parallelogram construction gluing in a cylinder. Now $b_{1}^{\prime}=b_{1}^{\prime \prime}=1$ is odd. Thus we get $N=\left(b_{1}^{\prime}+1\right)\left(b_{1}^{\prime \prime}+1\right)=4$ surfaces, but by lemma 14.4 only $N / 2=2$ of them have odd parity of the spin structure. There is also the $\gamma \rightarrow-\gamma$ symmetry.

$$
c=2 \cdot \frac{(5-1) !}{(7-2) !} \cdot \frac{\operatorname{Vol}\left(\mathcal{H}_{1}(1,1)\right)}{\operatorname{Vol}\left(\mathcal{H}_{1}^{\text {odd }}(2,2)\right)}=\frac{32}{15} \cdot \frac{1}{\zeta(2)} \approx 1.297
$$

$\Rightarrow(\overline{0+0}) \Rightarrow(\overline{0+0}) \Rightarrow$

In the multiplicity 2 case we have a pair of cylinders. The two sides of each cylinder return to different zeroes. The result is a pair of tori, each with a single marked point. We perform the figure eight construction creating two pairs of circles to which we glue intervening cylinders. We have $|\Gamma|=\left|\Gamma_{-}\right|=2$.

$$
c=\frac{1}{2} \cdot \frac{1}{2^{2-1}} \cdot \frac{(2-1) !(2-1) !}{(7-2) !} \cdot \frac{\operatorname{Vol}\left(\mathcal{H}_{1}(0)\right) \operatorname{Vol}\left(\mathcal{H}_{1}(0)\right)}{\operatorname{Vol}\left(\mathcal{H}_{1}^{\text {odd }}(2,2)\right)}=\frac{1}{6} \cdot \frac{1}{\zeta(2)} \approx 0.1013
$$

\section{ACKNOWLEDGMENTS}

The third author wants to thank MPI für Mathematik at Bonn and IHES at Bures-sur-Yvette for their hospitality while preparation of this paper. 


\section{Appendix. Values of the Constants}

\section{Appendix A. Saddle Connections Joining Distinct Zeroes}

A.1. Connected Strata in Genus 4. In the tables below we consider all connected strata in genus $g=4$. For each stratum we present the complete list of all possible topological configurations of geodesic saddle connections. To make calculations traceable we present for every configuration the orders of possible symmetries $\Gamma$ and $\Gamma_{-}$, the combinatorial constant $M$, and the result: the Siegel-Veech constant in quadratic asymptotics for the number of saddle connections (closed geodesics) of that type. The notations were introduced in Section 9.2 and Section 12.3

\begin{tabular}{|c|c|c|c|c|c|}
\hline Stratum $\mathcal{H}(5,1)$. \\
\hline$(1+5) \succ$ & $|\Gamma-|$ & $|\Gamma|$ & $M$ & $c$ & $c$ approx. \\
\hline$(0+0) \succ(0+4) \succ$ & 1 & 1 & 7 & $\frac{4311167}{373248}$ & 11.5504 \\
\hline$(0+2) \succ(0+2) \succ$ & 1 & 1 & 5 & $\frac{38125}{93312}$ & 0.408576 \\
\hline & 1 & 2 & $\frac{9}{2}$ & $\frac{21}{512}$ & 0.0410156 \\
\hline
\end{tabular}

\begin{tabular}{|c|c|c|c|c|c|}
\hline \multicolumn{7}{|c|}{ Stratum $\mathcal{H}(4,1,1)}$. \\
\hline$(1+1,4) \succ$ & 2 & 1 & 3 & $\frac{2403}{616}$ & 3.90097 \\
\hline$(0+0) \succ(0+0,4) \succ$ & 2 & 1 & 1 & $\frac{61}{616}$ & 0.099026 \\
\hline$(0+0) \succ(0+3,1) \succ$ & 1 & 1 & 8 & $\frac{1024}{1925}$ & 0.531948 \\
\hline$(0+2) \succ(0+1,1) \succ$ & 1 & 1 & 12 & $\frac{108}{1375}$ & 0.0785455 \\
\hline
\end{tabular}

\begin{tabular}{|c|c|c|c|c|c|}
\hline$(1+2,3) \succ$ & 1 & 1 & 4 & $\frac{368}{63}$ & 5.84127 \\
\hline$(1+3,2) \succ$ & 1 & 1 & 5 & $\frac{55625}{7168}$ & 7.76018 \\
\hline$(2+3,1) \succ$ & 1 & 1 & 6 & $\frac{81}{7}$ & 11.5714 \\
\hline$(0+0) \succ(0+2,2) \succ$ & 1 & 1 & 3 & $\frac{765}{3584}$ & 0.213449 \\
\hline$(0+0) \succ(0+1,3) \succ$ & 1 & 1 & 2 & $\frac{10}{63}$ & 0.15873 \\
\hline$(0+0) \succ(1+2,1) \succ$ & 1 & 1 & 4 & $\frac{20}{63}$ & 0.31746 \\
\hline$(0+0,2) \succ(0+2) \succ$ & 1 & 1 & 3 & $\frac{27}{1024}$ & 0.0263672 \\
\hline$(0+2) \succ(1+0,1) \succ$ & 1 & 1 & 6 & $\frac{3}{64}$ & 0.046875 \\
\hline$(1+1) \succ(0+1,1) \succ$ & 1 & 1 & 6 & $\frac{3}{64}$ & 0.046875 \\
\hline$(0+0) \succ(0+0) \succ(0+1,1) \succ$ & 1 & 1 & 2 & $\frac{5}{288}$ & 0.0173611 \\
\hline
\end{tabular}


Configurations of geodesic saddle connections. Stratum $\mathcal{H}(3,1,1,1)$.

\begin{tabular}{|c|c|c|c|c|c|}
\hline Degeneration pattern & $|\Gamma-|$ & $|\Gamma|$ & $M$ & $c$ & $c$ approx. \\
\hline$(1+1,3,1) \succ$ & 2 & 1 & 9 & $\frac{729}{62}$ & 11.7581 \\
\hline$(1+3,1,1) \succ$ & 1 & 1 & 15 & $\frac{185625}{7936}$ & 23.3902 \\
\hline$(0+0) \succ(0+0,3,1) \succ$ & 2 & 1 & 3 & $\frac{15}{62}$ & 0.241935 \\
\hline$(0+0) \succ(0+2,1,1) \succ$ & 1 & 1 & 9 & $\frac{2025}{3968}$ & 0.510333 \\
\hline$(0+2) \succ(0+0,1,1) \succ$ & 1 & 1 & 9 & $\frac{405}{7936}$ & 0.0510333 \\
\hline$(0+1,1) \succ(0+1,1) \succ$ & 1 & 2 & 12 & $\frac{3}{62}$ & 0.0483871 \\
\hline
\end{tabular}

Stratum $\mathcal{H}(2,2,1,1)$

\begin{tabular}{|c|c|c|c|c|c|}
\hline$(1+1,2,2) \succ$ & 2 & 1 & 3 & $\frac{4101}{1048}$ & 3.91317 \\
\hline$(1+2,2,1) \succ$ & 1 & 1 & 16 & $\frac{3072}{131}$ & 23.4504 \\
\hline$(2+2,1,1) \succ$ & 2 & 1 & 5 & $\frac{6875}{786}$ & 8.74682 \\
\hline$(0+0) \succ(0+0,2,2) \succ$ & 2 & 1 & 1 & $\frac{85}{1048}$ & 0.0811069 \\
\hline$(0+0) \succ(0+1,2,1) \succ$ & 1 & 1 & 8 & $\frac{200}{393}$ & 0.508906 \\
\hline$(0+0) \succ(1+1,1,1) \succ$ & 2 & 1 & 3 & $\frac{25}{131}$ & 0.19084 \\
\hline$(0+0,2) \succ(0+0,2) \succ$ & 2 & 2 & 1 & $\frac{3}{524}$ & 0.00572519 \\
\hline$(0+0,2) \succ(0+1,1) \succ$ & 1 & 1 & 8 & $\frac{16}{393}$ & 0.0407125 \\
\hline$(1+1) \succ(0+0,1,1) \succ$ & 2 & 1 & 3 & $\frac{5}{262}$ & 0.019084 \\
\hline$(0+1,1) \succ(1+0,1) \succ$ & 2 & 1 & 8 & $\frac{128}{3537}$ & 0.0361889 \\
\hline$(0+0) \succ(0+0) \succ(0+0,1,1) \succ$ & 2 & 1 & 1 & $\frac{25}{3537}$ & 0.00706814 \\
\hline
\end{tabular}

Stratum $\mathcal{H}(2,1,1,1,1)$.

\begin{tabular}{|c|c|c|c|c|c|}
\hline$(1+1,2,1,1) \succ$ & 2 & 1 & 18 & $\frac{1179}{50}$ & 23.58 \\
\hline$(1+2,1,1,1) \succ$ & 1 & 1 & 16 & $\frac{15872}{675}$ & 23.5141 \\
\hline$(0+0) \succ(0+0,2,1,1) \succ$ & 2 & 1 & 6 & $\frac{2}{5}$ & 0.4 \\
\hline$(0+0) \succ(0+1,1,1,1) \succ$ & 1 & 1 & 8 & $\frac{56}{135}$ & 0.414815 \\
\hline$(0+0,2) \succ(0+0,1,1) \succ$ & 2 & 1 & 6 & $\frac{1}{50}$ & 0.02 \\
\hline$(0+0,1,1) \succ(0+1,1) \succ$ & 1 & 1 & 24 & $\frac{16}{225}$ & 0.0711111 \\
\hline
\end{tabular}


TABLE 8. Principal stratum $\mathcal{H}(1, \ldots, 1)$. Approximate values of the constants $c$ for saddle connections of multiplicity two joining a pair of distinct zeroes.

$$
\mathcal{H}(\overbrace{1, \ldots, 1}^{2 g_{1}-2}, 0+0) \succ \mathcal{H}(\overbrace{1, \ldots, 1}^{2 g_{2}-2}, 0+0) \succ \quad g_{1}+g_{2}=g
$$

\begin{tabular}{|c|c|c|c|c|c|c|}
\hline & $g_{2}=1$ & $g_{2}=2$ & $g_{2}=3$ & $g_{2}=4$ & $g_{2}=5$ & $g_{2}=6$ \\
\hline$g_{1}=1$ & 0.6250 & 0.8571 & 0.8355 & 0.8393 & 0.8426 & 0.8435 \\
\hline$g_{1}=2$ & 0.8571 & 0.07958 & 0.07193 & 0.04160 & 0.02708 & 0.01896 \\
\hline$g_{1}=3$ & 0.8355 & 0.07193 & 0.009358 & 0.007018 & 0.003195 & 0.001651 \\
\hline$g_{1}=4$ & 0.8393 & 0.04160 & 0.007018 & $0.9204 \cdot 10^{-3}$ & $0.6185 \cdot 10^{-3}$ & $0.2454 \cdot 10^{-3}$ \\
\hline$g_{1}=5$ & 0.8426 & 0.02708 & 0.003195 & $0.6185 \cdot 10^{-3}$ & $0.7978 \cdot 10^{-4}$ & $0.5011 \cdot 10^{-4}$ \\
\hline$g_{1}=6$ & 0.8435 & 0.01896 & 0.001651 & $0.2454 \cdot 10^{-3}$ & $0.5011 \cdot 10^{-4}$ & $0.6383 \cdot 10^{-5}$ \\
\hline$g_{1}=7$ & 0.8430 & 0.01398 & $0.9350 \cdot 10^{-3}$ & $0.1100 \cdot 10^{-3}$ & $0.1822 \cdot 10^{-4}$ & $0.3840 \cdot 10^{-5}$ \\
\hline$g_{1}=8$ & 0.8418 & 0.01072 & $0.5675 \cdot 10^{-3}$ & $0.5415 \cdot 10^{-4}$ & $0.7420 \cdot 10^{-5}$ & $0.1315 \cdot 10^{-5}$ \\
\hline$g_{1}=9$ & 0.8406 & 0.008475 & $0.3638 \cdot 10^{-3}$ & $0.2872 \cdot 10^{-4}$ & $0.3309 \cdot 10^{-5}$ & $0.5001 \cdot 10^{-6}$ \\
\hline$g_{1}=10$ & 0.8393 & 0.006864 & $0.2438 \cdot 10^{-3}$ & $0.1618 \cdot 10^{-4}$ & $0.1590 \cdot 10^{-5}$ & $0.2072 \cdot 10^{-6}$ \\
\hline$g_{1}=11$ & 0.8382 & 0.005670 & $0.1694 \cdot 10^{-3}$ & $0.9585 \cdot 10^{-5}$ & $0.8122 \cdot 10^{-6}$ & $0.9226 \cdot 10^{-7}$ \\
\hline$g_{1}=12$ & 0.8372 & 0.004762 & $0.1213 \cdot 10^{-3}$ & $0.5921 \cdot 10^{-5}$ & $0.4372 \cdot 10^{-6}$ & $0.4366 \cdot 10^{-7}$ \\
\hline$g_{1}=13$ & 0.8363 & 0.004056 & $0.8909 \cdot 10^{-4}$ & $0.3790 \cdot 10^{-5}$ & $0.2461 \cdot 10^{-6}$ & $0.2177 \cdot 10^{-7}$ \\
\hline$g_{1}=14$ & 0.8354 & 0.003495 & $0.6691 \cdot 10^{-4}$ & $0.2503 \cdot 10^{-5}$ & $0.1440 \cdot 10^{-6}$ & $0.1136 \cdot 10^{-7}$ \\
\hline$g_{1}=15$ & 0.8347 & 0.003043 & $0.5122 \cdot 10^{-4}$ & $0.1697 \cdot 10^{-5}$ & $0.8712 \cdot 10^{-7}$ & $0.6173 \cdot 10^{-8}$ \\
\hline$g_{1}=16$ & 0.8340 & 0.002673 & $0.3986 \cdot 10^{-4}$ & $0.1179 \cdot 10^{-5}$ & $0.5431 \cdot 10^{-7}$ & $0.3474 \cdot 10^{-8}$ \\
\hline$g_{1}=$ & 0.8334 & 0.002367 & $0.3149 \cdot 10^{-4}$ & $0.8358 \cdot 10^{-6}$ & $0.3476 \cdot 10^{-7}$ & $0.2017 \cdot 10^{-8}$ \\
\hline$g_{1}=18$ & 0.8329 & 0.002110 & $0.2520 \cdot 10^{-4}$ & $0.6038 \cdot 10^{-6}$ & $0.2278 \cdot 10^{-7}$ & $0.1204 \cdot 10^{-8}$ \\
\hline$g_{1}=19$ & 0.8324 & 0.001893 & $0.2041 \cdot 10^{-4}$ & $0.4436 \cdot 10^{-6}$ & $0.1525 \cdot 10^{-7}$ & $0.7378 \cdot 10^{-9}$ \\
\hline$g_{1}=20$ & 0.8319 & 0.001708 & $0.1670 \cdot 10^{-4}$ & $0.3308 \cdot 10^{-6}$ & $0.1041 \cdot 10^{-7}$ & $0.4624 \cdot 10^{-9}$ \\
\hline$g_{1}=21$ & 0.8315 & 0.001549 & $0.1380 \cdot 10^{-4}$ & $0.2501 \cdot 10^{-6}$ & $0.7226 \cdot 10^{-8}$ & $0.2960 \cdot 10^{-9}$ \\
\hline$g_{1}=22$ & 0.8312 & 0.001411 & $0.1150 \cdot 10^{-4}$ & $0.1915 \cdot 10^{-6}$ & $0.5098 \cdot 10^{-8}$ & $0.1931 \cdot 10^{-9}$ \\
\hline$g_{1}=23$ & 0.8308 & 0.001290 & $0.9663 \cdot 10^{-5}$ & $0.1482 \cdot 10^{-6}$ & $0.3650 \cdot 10^{-8}$ & $0.1282 \cdot 10^{-9}$ \\
\hline$g_{1}=24$ & 0.8305 & 0.001185 & $0.8177 \cdot 10^{-5}$ & $0.1160 \cdot 10^{-6}$ & $0.2648 \cdot 10^{-8}$ & $0.8649 \cdot 10^{-10}$ \\
\hline$g_{1}=25$ & 0.8302 & 0.00 & $0.6966 \cdot 10^{-5}$ & $0.9163 \cdot 10^{-7}$ & $0.1946 \cdot 10^{-8}$ & $0.5923 \cdot 10^{-10}$ \\
\hline$g_{1}=26$ & 0.8299 & 0.001009 & $0.5971 \cdot 10^{-5}$ & $0.7304 \cdot 10^{-7}$ & $0.1446 \cdot 10^{-8}$ & $0.4113 \cdot 10^{-10}$ \\
\hline$g_{1}=27$ & 0.8297 & $0.9353 \cdot 10^{-3}$ & $0.5147 \cdot 10^{-5}$ & $0.5870 \cdot 10^{-7}$ & $0.1086 \cdot 10^{-8}$ & $0.2893 \cdot 10^{-10}$ \\
\hline$g_{1}=28$ & 0.8294 & $0.8695 \cdot 10^{-3}$ & $0.4461 \cdot 10^{-5}$ & $0.4754 \cdot 10^{-7}$ & $0.8236 \cdot 10^{-9}$ & $0.2060 \cdot 10^{-10}$ \\
\hline$g_{1}=29$ & 0.8292 & $0.8104 \cdot 10^{-3}$ & $0.3885 \cdot 10^{-5}$ & $0.3878 \cdot 10^{-7}$ & $0.6305 \cdot 10^{-9}$ & $0.1483 \cdot 10^{-10}$ \\
\hline$g_{1}=30$ & 0.8290 & $0.7571 \cdot 10^{-3}$ & $0.3400 \cdot 10^{-5}$ & $0.3184 \cdot 10^{-7}$ & $0.4869 \cdot 10^{-9}$ & $0.1079 \cdot 10^{-10}$ \\
\hline
\end{tabular}


TABle 9. Connected component $\mathcal{H}^{\text {hyp }}(g-1, g-1)$; approximate values of the constants for saddle connections of multiplicity two joining distinct zeroes; $g_{1}+g_{2}=g$

$$
\left.\mathcal{H}_{1}^{\text {hyp }}\left(\overline{\left(g_{1}-1\right.}\right)+\left(\overline{g_{1}-1}\right)\right) \succ \mathcal{H}_{1}^{\text {hyp }}\left(\overline{\left(g_{2}-1\right)}+\overline{\left(g_{2}-1\right)}\right) \succ
$$

\begin{tabular}{|c|c|c|c|c|c|c|c|c|c|c|}
\hline & $g_{2}=1$ & $g_{2}=2$ & $g_{2}=3$ & $g_{2}=4$ & $g_{2}=5$ & $g_{2}=6$ & $g_{2}=7$ & $g_{2}=8$ & $g_{2}=9$ & $g_{2}=10$ \\
\hline$g_{1}=1$ & 0.6250 & 1.9687 & 2.9297 & 4.1122 & 5.5112 & 7.1245 & 8.9513 & 10.991 & 13.244 & 15.709 \\
\hline$g_{1}=2$ & 1.9687 & 1.1074 & 2.7191 & 3.3679 & 4.1334 & 5.0053 & 5.9792 & 7.0526 & 8.2244 & 9.4935 \\
\hline$g_{1}=3$ & .9297 & 2.7191 & 1.4729 & 3.3412 & 3.8411 & 4.4225 & 5.0749 & 5.7932 & 6.5745 & 7.4168 \\
\hline$g_{1}=4$ & 4.1122 & 3.3679 & 3.3412 & 1.7750 & 3.8803 & 4.2917 & 4.7662 & 5.2948 & 5.8725 & 6.4961 \\
\hline$g_{1}=5$ & 5.5112 & 4.1334 & 3.8411 & 3.8803 & 2.0372 & 4.3611 & 4.7134 & 5.1173 & 5.5653 & 6.0526 \\
\hline$g_{1}=6$ & 7.1245 & 5.0053 & 4.4225 & 4.2917 & 4.3611 & 2.2714 & 4.7983 & 5.1083 & 5.4619 & 5.8527 \\
\hline$g_{1}=7$ & 8.9513 & 5.9792 & 5.0749 & 4.7662 & 4.7134 & 4.7983 & 2.4849 & 5.2016 & 5.4798 & 5.7955 \\
\hline$g_{1}=8$ & 10.991 & 7.0526 & 5.7932 & 5.2948 & 5.1173 & 5.1083 & 5.2016 & 2.6821 & 5.5776 & 5.8309 \\
\hline$g_{1}=9$ & 13.244 & 8.2244 & 6.5745 & 5.8725 & 5.5653 & 5.4619 & 5.4798 & 5.5776 & 2.8663 & 5.9309 \\
\hline$g_{1}=10$ & 15.709 & 9.4935 & 7.4168 & 6.4961 & 6.0526 & 5.8527 & 5.7955 & 5.8309 & 5.9309 & 3.0396 \\
\hline$g_{1}=11$ & 18.387 & 10.860 & 8.3192 & 7.1637 & 6.5763 & 6.2764 & 6.1434 & 6.1170 & 6.1642 & 6.2652 \\
\hline$g_{1}=12$ & 21.277 & 12.322 & 9.2807 & 7.8740 & 7.1344 & 6.7305 & 6.5198 & 6.4315 & 6.4266 & 6.4821 \\
\hline$g_{1}=13$ & 79 & 31 & 10 & 59 & 55 & 28 & 21 & 710 & 143 & 6.7250 \\
\hline$g_{1}=14$ & 27.694 & 15.536 & 11.379 & 9.4190 & 8.3487 & 7.7223 & 7.3487 & 7.1334 & 7.0243 & 6.9906 \\
\hline$g_{1}=15$ & 31.221 & 17.288 & 12.516 & 10.253 & 9.0032 & 8.2577 & 7.7983 & 7.5170 & 7.3547 & 7.2764 \\
\hline$g_{1}=16$ & 34.961 & 19.135 & 13.710 & 11.127 & 9.6885 & 8.8184 & 8.2697 & 7.9205 & 7.7040 & 7.5806 \\
\hline$g_{1}=17$ & 38.913 & 21.078 & 14.962 & 12.041 & 10.404 & 9.4038 & 8.7624 & 8.3431 & 8.0710 & 7.9019 \\
\hline$g_{1}=18$ & 43.077 & 23.116 & 16.271 & 12.994 & 11.150 & 10.014 & 9.2757 & 8.7839 & 8.4548 & 8.2390 \\
\hline$g_{1}=19$ & 47.453 & 25.251 & 17.638 & 13.987 & 11.926 & 10.647 & 9.8091 & 9.2425 & 8.8547 & 8.5913 \\
\hline$g_{1}=20$ & 52.042 & 27.481 & 19.062 & 15.020 & 12.731 & 11.304 & 10.362 & 9.7183 & 9.2702 & 8.9580 \\
\hline$g_{1}=21$ & 56.842 & 29.807 & 20.543 & 16.092 & 13.566 & 11.985 & 10.935 & 10.211 & 9.7007 & 9.3385 \\
\hline$g_{1}=22$ & 61.856 & 32.229 & 22.081 & 17.203 & 14.430 & 12.689 & 11.527 & 10.720 & 10.146 & 9.7325 \\
\hline$g_{1}=23$ & 67.081 & 34.746 & 23.677 & 18.354 & 15.323 & 13.416 & 12.138 & 11.246 & 10.606 & 10.140 \\
\hline$g_{1}=24$ & 72.518 & 37.359 & 25.329 & 19.544 & 16.245 & 14.166 & 12.768 & 11.788 & 11.079 & 10.559 \\
\hline$g_{1}=25$ & 78.168 & 40.067 & 27.039 & 20.772 & 17.197 & 14.939 & 13.417 & 12.345 & 11.567 & 10.992 \\
\hline$g_{1}=26$ & 84.030 & 42.871 & 28.806 & 22.040 & 18.177 & 15.735 & 14.085 & 12.919 & 12.069 & 11.436 \\
\hline$g_{1}=27$ & 90.104 & 45.770 & 30.630 & 23.347 & 19.186 & 16.553 & 14.771 & 13.508 & 12.584 & 11.893 \\
\hline$g_{1}=28$ & 96.391 & 48.766 & 32.510 & 24.692 & 20.224 & 17.394 & 15.475 & 14.113 & 13.113 & 12.361 \\
\hline$g_{1}=29$ & 102.89 & 51.856 & 34.448 & 26.077 & 21.291 & 18.257 & 16.198 & 14.733 & 13.655 & 12.842 \\
\hline$g_{1}=30$ & 109.60 & 55.042 & 36.443 & 27.500 & 22.387 & 19.143 & 16.939 & 15.369 & 14.210 & 13.334 \\
\hline
\end{tabular}




\section{Appendix B. Saddle Connections Joining a Zero to Itself}

B.1. Connected Strata in Genus 4. In the tables below we consider all connected strata in genus $g=4$. For each stratum we present the complete list of admissible constructions ( = all possible topological configurations of homologous closed geodesics). To make calculations traceable we present for every configuration the orders of possible symmetries $\Gamma$ and $\Gamma_{-}$, the combinatorial constant $M$, and the result: the constant in quadratic asymptotics for the number of saddle connections (closed geodesics) of this type.

Stratum $\mathcal{H}(5,1)$

Configurations of closed geodesics.

\begin{tabular}{|c|c|c|c|c|c|}
\hline Degeneration pattern & $\left|\Gamma_{-}\right|$ & $|\Gamma|$ & $M$ & $c \cdot \zeta(2)$ & $c \cdot \zeta(2)$ approx. \\
\hline$\Rightarrow(\overline{\mathbf{0}}, \overline{\mathbf{4}}) \Rightarrow$ & 1 & 1 & 5 & $\frac{38125}{15552}$ & 2.45145 \\
\hline$\rightarrow(\overline{\mathbf{0}}, \overline{\mathbf{3}} ; 1) \rightarrow$ & 1 & 1 & 4 & $\frac{2240}{243}$ & 9.21811 \\
\hline$\Rightarrow(\overline{\mathbf{0}+\mathbf{3}} ; 1) \Rightarrow$ & 1 & 1 & 4 & $\frac{320}{243}$ & 1.31687 \\
\hline$\Rightarrow(\overline{\mathbf{1 + 2}} ; 1) \Rightarrow$ & 1 & 1 & 4 & $\frac{320}{243}$ & 1.31687 \\
\hline$\rightarrow(\overline{\mathbf{1}}, \overline{\mathbf{2}} ; 1) \rightarrow$ & 1 & 1 & 6 & $\frac{175}{18}$ & 9.72222 \\
\hline$\rightarrow(\overline{\mathbf{0}+\mathbf{0}}) \Rightarrow(\overline{\mathbf{0}}, \overline{\mathbf{2}}) \rightarrow$ & 1 & 1 & 3 & $\frac{35}{288}$ & 0.121528 \\
\hline$\rightarrow(\overline{\mathbf{0}}, \overline{\mathbf{0}}) \Rightarrow(\overline{\mathbf{0}+\mathbf{2}}) \rightarrow$ & 1 & 1 & 3 & $\frac{35}{576}$ & 0.0607639 \\
\hline$\rightarrow(\overline{\mathbf{0}}, \overline{\mathbf{0}}) \Rightarrow(\overline{\mathbf{1}+\mathbf{1}}) \rightarrow$ & 1 & 1 & 3 & $\frac{35}{576}$ & 0.0607639 \\
\hline$\rightarrow(\overline{\mathbf{0}}, \overline{\mathbf{0}}) \Rightarrow(\overline{\mathbf{2}+\mathbf{0}}) \rightarrow$ & 1 & 1 & 3 & $\frac{35}{576}$ & 0.0607639 \\
\hline$\rightarrow(\overline{\mathbf{0}+\mathbf{0}}) \rightarrow(\overline{\mathbf{0}}, \overline{\mathbf{1}} ; 1) \rightarrow$ & 1 & 1 & 2 & $\frac{175}{486}$ & 0.360082 \\
\hline$\rightarrow(\overline{\mathbf{0}}, \overline{\mathbf{0}}) \rightarrow(\overline{\mathbf{0}+\mathbf{1}} ; 1) \rightarrow$ & 1 & 1 & 2 & $\frac{35}{243}$ & 0.144033 \\
\hline$\rightarrow(\overline{\mathbf{0 + 0}}) \Rightarrow(\overline{\mathbf{0 + 1}} ; 1) \rightarrow$ & 1 & 1 & 2 & $\frac{35}{486}$ & 0.0720165 \\
\hline$\rightarrow(\overline{0+0}) \Rightarrow(\overline{1+0} ; 1) \rightarrow$ & 1 & 1 & 2 & $\frac{35}{486}$ & 0.0720165 \\
\hline$\rightarrow(\overline{\mathbf{0}+\mathbf{0}}) \rightarrow(\overline{\mathbf{0}+\mathbf{0}}) \Rightarrow(\overline{\mathbf{0}}, \overline{\mathbf{0}}) \rightarrow$ & 1 & 1 & 1 & $\frac{175}{7776}$ & 0.0225051 \\
\hline
\end{tabular}


Stratum $\mathcal{H}(4,1,1)$.

Configurations of closed geodesics.

\begin{tabular}{|c|c|c|c|c|c|}
\hline Degeneration pattern & $\left|\Gamma_{-}\right|$ & $|\Gamma|$ & $M$ & $c \cdot \zeta(2)$ & $c \cdot \zeta(2)$ approx. \\
\hline$\Rightarrow(\overline{\mathbf{0}}, \overline{\mathbf{0}} ; 4) \Rightarrow$ & 2 & 1 & 1 & $\frac{61}{88}$ & 0.693182 \\
\hline$\Rightarrow(\overline{\mathbf{0}}, \overline{\mathbf{3}} ; 1) \Rightarrow$ & 1 & 1 & 8 & $\frac{1024}{275}$ & 3.72364 \\
\hline$\rightarrow(\overline{\mathbf{0}}, \overline{\mathbf{2}} ; 1,1) \rightarrow$ & 1 & 1 & 3 & $\frac{432}{55}$ & 7.85455 \\
\hline$\Rightarrow(\overline{\mathbf{0}+\mathbf{2}} ; 1,1) \Rightarrow$ & 1 & 1 & 3 & $\frac{54}{55}$ & 0.981818 \\
\hline$\Rightarrow(\overline{\mathbf{1}+\mathbf{1}} ; 1,1) \Rightarrow$ & 2 & 1 & $\frac{3}{2}$ & $\frac{27}{55}$ & 0.490909 \\
\hline$\rightarrow(\overline{\mathbf{1}}, \overline{\mathbf{1}} ; 1,1) \rightarrow$ & 2 & 1 & 2 & $\frac{224}{55}$ & 4.07273 \\
\hline$\rightarrow(\overline{\mathbf{0}}, \overline{\mathbf{0}}) \Rightarrow(\overline{\mathbf{0}}, \overline{\mathbf{2}}) \rightarrow$ & 1 & 1 & 6 & $\frac{27}{275}$ & 0.0981818 \\
\hline$\Rightarrow(\overline{\mathbf{0}}, \overline{\mathbf{0}}) \Rightarrow(\overline{\mathbf{0}+\mathbf{2}}) \Rightarrow$ & 1 & 1 & 6 & $\frac{27}{1100}$ & 0.0245455 \\
\hline$\Rightarrow(\overline{\mathbf{0}}, \overline{\mathbf{0}}) \Rightarrow(\overline{\mathbf{1}+\mathbf{1}}) \Rightarrow$ & 2 & 1 & 3 & $\frac{27}{2200}$ & 0.0122727 \\
\hline$\rightarrow(\overline{\mathbf{0}+\mathbf{0}}) \rightarrow(\overline{\mathbf{0}}, \overline{\mathbf{0}} ; 1,1) \rightarrow$ & 2 & 1 & $\frac{1}{2}$ & $\frac{6}{55}$ & 0.109091 \\
\hline$\rightarrow(\overline{\mathbf{0}}, \overline{\mathbf{0}}) \rightarrow(\overline{\mathbf{0}+\mathbf{0}} ; 1,1) \rightarrow$ & 2 & 1 & $\frac{1}{2}$ & $\frac{2}{55}$ & 0.0363636 \\
\hline$\rightarrow(\overline{\mathbf{0}+\mathbf{0}}) \Rightarrow(\overline{\mathbf{0}+\mathbf{0}} ; 1,1) \rightarrow$ & 1 & 1 & 1 & $\frac{2}{55}$ & 0.0363636 \\
\hline$\rightarrow(\overline{\mathbf{0}+\mathbf{0}}) \Rightarrow(\overline{\mathbf{0}}, \overline{\mathbf{1}} ; 1) \rightarrow$ & 1 & 1 & 4 & $\frac{8}{55}$ & 0.145455 \\
\hline$\rightarrow(\overline{\mathbf{0}}, \overline{\mathbf{0}}) \Rightarrow(\overline{\mathbf{0}+\mathbf{1}} ; 1) \rightarrow$ & 1 & 1 & 4 & $\frac{16}{275}$ & 0.0581818 \\
\hline$\rightarrow(\overline{\mathbf{0}}, \overline{\mathbf{0}}) \Rightarrow(\overline{\mathbf{1}+\mathbf{0}} ; 1) \rightarrow$ & 1 & 1 & 4 & $\frac{16}{275}$ & 0.0581818 \\
\hline$\overline{\mathbf{0}+\mathbf{0}}) \rightarrow(\overline{\mathbf{0}}, \overline{\mathbf{0}}) \Rightarrow(\overline{\mathbf{0}}, \overline{\mathbf{0}}) \rightarrow$ & 2 & 1 & 1 & $\frac{1}{110}$ & 0.00909091 \\
\hline$\overline{\mathbf{0}+\mathbf{0}}) \Rightarrow(\overline{\mathbf{0}}, \overline{\mathbf{0}}) \Rightarrow(\overline{\mathbf{0}+\mathbf{0}}) \rightarrow$ & 2 & 1 & 1 & $\frac{1}{220}$ & 0.00454545 \\
\hline
\end{tabular}


Stratum $\mathcal{H}(3,2,1)$

Configurations of closed geodesics.

\begin{tabular}{|c|c|c|c|c|c|}
\hline Degeneration pattern & $\left|\Gamma_{-}\right|$ & $|\Gamma|$ & $M$ & $c \cdot \zeta(2)$ & $c \cdot \zeta(2)$ approx. \\
\hline$\Rightarrow(\overline{\mathbf{0}}, \overline{\mathbf{2}} ; 2) \Rightarrow$ & 1 & 1 & 3 & $\frac{765}{512}$ & 1.49414 \\
\hline$\rightarrow(\overline{\mathbf{0}}, \overline{\mathbf{0}} ; 3,1) \rightarrow$ & 2 & 1 & $\frac{1}{2}$ & $\frac{20}{9}$ & 2.22222 \\
\hline$\Rightarrow(\overline{\mathbf{0}+\mathbf{0}} ; 3,1) \Rightarrow$ & 2 & 1 & $\frac{1}{2}$ & $\frac{5}{18}$ & 0.277778 \\
\hline$\Rightarrow(\overline{\mathbf{0}}, \overline{\mathbf{1}} ; 3) \Rightarrow$ & 1 & 1 & 2 & $\frac{10}{9}$ & 1.11111 \\
\hline$\rightarrow(\overline{\mathbf{0}}, \overline{\mathbf{1}} ; 2,1) \rightarrow$ & 1 & 1 & 2 & $\frac{25}{4}$ & 6.25 \\
\hline$\Rightarrow(\overline{\mathbf{0}+\mathbf{1}} ; 2,1) \Rightarrow$ & 1 & 1 & 2 & $\frac{25}{32}$ & 0.78125 \\
\hline$\Rightarrow(\overline{\mathbf{1}}, \overline{\mathbf{2}} ; 1) \Rightarrow$ & 1 & 1 & 6 & $\frac{75}{32}$ & 2.34375 \\
\hline$\rightarrow(\overline{\mathbf{0}+\mathbf{0}}) \Rightarrow(\overline{\mathbf{0}}, \overline{\mathbf{0}} ; 2) \rightarrow$ & 1 & 1 & 1 & $\frac{25}{512}$ & 0.0488281 \\
\hline$\rightarrow(\overline{\mathbf{0}}, \overline{\mathbf{0}}) \Rightarrow(\overline{\mathbf{0}}+\mathbf{0} ; 2) \rightarrow$ & 1 & 1 & 1 & $\frac{5}{256}$ & 0.0195313 \\
\hline$\rightarrow(\overline{\mathbf{0}}, \overline{\mathbf{0}}) \Rightarrow(\overline{\mathbf{2}}, \overline{\mathbf{0}}) \rightarrow$ & 1 & 1 & 3 & $\frac{15}{256}$ & 0.0585938 \\
\hline$\Rightarrow(\overline{\mathbf{0}+\mathbf{0}}) \Rightarrow(\overline{\mathbf{0}}, \overline{\mathbf{2}}) \Rightarrow$ & 1 & 1 & 3 & $\frac{15}{512}$ & 0.0292969 \\
\hline$\rightarrow(\overline{\mathbf{0}}, \overline{\mathbf{0}}) \rightarrow(\overline{\mathbf{0}}, \overline{\mathbf{1}} ; 1) \rightarrow$ & 1 & 1 & 2 & $\frac{25}{144}$ & 0.173611 \\
\hline$\rightarrow(\overline{\mathbf{0}+\mathbf{0}}) \Rightarrow(\overline{\mathbf{1}}, \overline{\mathbf{0}} ; 1) \rightarrow$ & 1 & 1 & 2 & $\frac{25}{288}$ & 0.0868056 \\
\hline$\rightarrow(\overline{\mathbf{0}}, \overline{\mathbf{0}}) \Rightarrow(\overline{\mathbf{1}}, \overline{\mathbf{1}}) \rightarrow$ & 1 & 1 & 4 & $\frac{5}{72}$ & 0.0694444 \\
\hline$\overline{\mathbf{0}+\mathbf{0}}) \Rightarrow(\overline{\mathbf{0}+\mathbf{1}} ; 1) \Rightarrow$ & 1 & 1 & 2 & $\frac{5}{288}$ & 0.0173611 \\
\hline $\boldsymbol{\mathbf { 0 }}, \overline{\mathbf{0}}) \rightarrow(\overline{\mathbf{0}+\mathbf{0}}) \Rightarrow(\overline{\mathbf{0}}, \overline{\mathbf{0}}) \rightarrow$ & 1 & 1 & 1 & $\frac{25}{2304}$ & 0.0108507 \\
\hline$\Rightarrow(\overline{\mathbf{0}+\mathbf{0}}) \Rightarrow(\overline{\mathbf{0}}, \overline{\mathbf{0}}) \rightarrow$ & 1 & 1 & 1 & $\frac{25}{4608}$ & 0.00542535 \\
\hline
\end{tabular}


Stratum $\mathcal{H}(3,1,1,1)$.

Configurations of closed geodesics.

\begin{tabular}{|c|c|c|c|c|c|}
\hline Degeneration pattern & $\left|\Gamma_{-}\right|$ & $|\Gamma|$ & $M$ & $c \cdot \zeta(2)$ & $c \cdot \zeta(2)$ approx. \\
\hline$\Rightarrow(\overline{\mathbf{0}}, \overline{\mathbf{0}} ; 3,1) \Rightarrow$ & 2 & 1 & 3 & $\frac{60}{31}$ & 1.93548 \\
\hline$\Rightarrow(\overline{\mathbf{0}}, \overline{\mathbf{2}} ; 1,1) \Rightarrow$ & 1 & 1 & 9 & $\frac{2025}{496}$ & 4.08266 \\
\hline$\rightarrow(\overline{\mathbf{0}}, \overline{\mathbf{1}} ; 1,1,1) \rightarrow$ & 1 & 1 & 2 & $\frac{1575}{248}$ & 6.35081 \\
\hline$\Rightarrow(\overline{\mathbf{0}+\mathbf{1}} ; 1,1,1) \Rightarrow$ & 1 & 1 & 2 & $\frac{175}{248}$ & 0.705645 \\
\hline$\Rightarrow(\overline{\mathbf{0}}, \overline{\mathbf{0}}) \Rightarrow(\overline{\mathbf{0}}, \overline{\mathbf{2}}) \Rightarrow$ & 1 & 1 & 18 & $\frac{405}{7936}$ & 0.0510333 \\
\hline$\rightarrow(\overline{\mathbf{0}+\mathbf{0}}) \Rightarrow(\overline{\mathbf{0}}, \overline{\mathbf{0}} ; 1,1) \rightarrow$ & 1 & 1 & 3 & $\frac{225}{1984}$ & 0.113407 \\
\hline$\rightarrow(\overline{\mathbf{0}}, \overline{\mathbf{0}}) \Rightarrow(\overline{\mathbf{0}+\mathbf{0}} ; 1,1) \rightarrow$ & 1 & 1 & 3 & $\frac{75}{1984}$ & 0.0378024 \\
\hline$\rightarrow(\overline{\mathbf{0}}, \overline{\mathbf{0}}) \Rightarrow(\overline{\mathbf{0}}, \mathbf{1} ; 1) \rightarrow$ & 1 & 1 & 12 & $\frac{75}{496}$ & 0.15121 \\
\hline$\Rightarrow(\overline{\mathbf{0}}, \overline{\mathbf{0}}) \Rightarrow(\overline{\mathbf{0}+\mathbf{1}} ; 1) \Rightarrow$ & 1 & 1 & 12 & $\frac{15}{496}$ & 0.0302419 \\
\hline $\mathbf{( \overline { \mathbf { 0 } } \mathbf { + }}) \Rightarrow(\overline{\mathbf{0}}, \overline{\mathbf{0}}) \Rightarrow(\overline{\mathbf{0}}, \overline{\mathbf{0}}) \rightarrow$ & 1 & 1 & 6 & $\frac{75}{7936}$ & 0.0094506 \\
\hline
\end{tabular}


Stratum $\mathcal{H}(2,2,1,1)$

Configurations of closed geodesics.

\begin{tabular}{|c|c|c|c|c|c|}
\hline Degeneration pattern & $\left|\Gamma_{-}\right|$ & $|\Gamma|$ & $M$ & $c \cdot \zeta(2)$ & $c \cdot \zeta(2)$ approx. \\
\hline$\Rightarrow(\overline{\mathbf{0}}, \overline{\mathbf{0}} ; 2,2) \Rightarrow$ & 2 & 1 & 1 & $\frac{85}{131}$ & 0.648855 \\
\hline$\rightarrow(\overline{\mathbf{0}}, \overline{\mathbf{0}} ; 2,1,1) \rightarrow$ & 2 & 1 & 1 & $\frac{600}{131}$ & 4.58015 \\
\hline$\Rightarrow(\overline{\mathbf{0}+\mathbf{0}} ; 2,1,1) \Rightarrow$ & 2 & 1 & 1 & $\frac{200}{393}$ & 0.508906 \\
\hline$\Rightarrow(\overline{\mathbf{0}}, \overline{\mathbf{1}} ; 2,1) \Rightarrow$ & 1 & 1 & 8 & $\frac{1600}{393}$ & 4.07125 \\
\hline$\Rightarrow(\overline{\mathbf{1}}, \overline{\mathbf{1}} ; 1,1) \Rightarrow$ & 2 & 1 & 4 & $\frac{5600}{3537}$ & 1.58326 \\
\hline$\rightarrow(\overline{\mathbf{0}}, \overline{\mathbf{0}}) \Rightarrow(\overline{\mathbf{0}}, \overline{\mathbf{0}} ; 2) \rightarrow$ & 1 & 1 & 4 & $\frac{25}{393}$ & 0.0636132 \\
\hline$\Rightarrow(\overline{\mathbf{0}+\mathbf{0}}) \Rightarrow(\overline{\mathbf{0}}, \overline{\mathbf{0}} ; 2) \Rightarrow$ & 2 & 1 & 2 & $\frac{25}{1572}$ & 0.0159033 \\
\hline$\Rightarrow(\overline{\mathbf{0}}, \overline{\mathbf{0}}) \Rightarrow(\overline{\mathbf{0}+\mathbf{0}} ; 2) \Rightarrow$ & 2 & 1 & 2 & $\frac{5}{786}$ & 0.00636132 \\
\hline$\rightarrow(\overline{\mathbf{0}}, \overline{\mathbf{0}}) \rightarrow(\overline{\mathbf{0}}, \overline{\mathbf{0}} ; 1,1) \rightarrow$ & 2 & 1 & 1 & $\frac{100}{1179}$ & 0.0848176 \\
\hline$\rightarrow(\overline{\mathbf{0}}, \overline{\mathbf{0}}) \Rightarrow(\overline{\mathbf{1}}, \overline{\mathbf{0}} ; 1) \rightarrow$ & 1 & 1 & 8 & $\frac{400}{3537}$ & 0.11309 \\
\hline$\Rightarrow(\overline{\mathbf{0}+\mathbf{0}}) \Rightarrow(\overline{\mathbf{0}+\mathbf{0}} ; 1,1) \Rightarrow$ & 2 & 1 & 1 & $\frac{25}{3537}$ & 0.00706814 \\
\hline$\Rightarrow(\overline{\mathbf{0}+\mathbf{0}}) \Rightarrow(\overline{\mathbf{0}}, \overline{\mathbf{1}} ; 1) \Rightarrow$ & 1 & 1 & 8 & $\frac{200}{3537}$ & 0.0565451 \\
\hline$\Rightarrow(\overline{\mathbf{0}}, \overline{\mathbf{0}}) \Rightarrow(\overline{\mathbf{1}}, \overline{\mathbf{1}}) \Rightarrow$ & 2 & 1 & 8 & $\frac{80}{3537}$ & 0.022618 \\
\hline$\rightarrow(\overline{\mathbf{0}}, \overline{\mathbf{0}}) \rightarrow(\overline{\mathbf{0}}, \overline{\mathbf{0}}) \Rightarrow(\overline{\mathbf{0}}, \overline{\mathbf{0}}) \rightarrow$ & 2 & 1 & 2 & $\frac{25}{3537}$ & 0.00706814 \\
\hline $\boldsymbol{\mathbf { 0 }}, \overline{\mathbf{0}}) \Rightarrow(\overline{\mathbf{0}+\mathbf{0}}) \Rightarrow(\overline{\mathbf{0}}, \overline{\mathbf{0}}) \rightarrow$ & 2 & 1 & 2 & $\frac{25}{7074}$ & 0.00353407 \\
\hline$\Rightarrow(\overline{\mathbf{0}+\mathbf{0}}) \Rightarrow(\overline{\mathbf{0}}, \overline{\mathbf{0}}) \Rightarrow$ & 2 & 1 & 2 & $\frac{25}{14148}$ & 0.00176703 \\
\hline
\end{tabular}


Stratum $\mathcal{H}(2,1,1,1,1)$.

Configurations of closed geodesics.

\begin{tabular}{|c|c|c|c|c|c|}
\hline Degeneration pattern & $\left|\Gamma_{-}\right|$ & $|\Gamma|$ & $M$ & $c \cdot \zeta(2)$ & $c \cdot \zeta(2)$ approx. \\
\hline$\Rightarrow(\overline{\mathbf{0}}, \overline{\mathbf{0}} ; 2,1,1) \Rightarrow$ & 2 & 1 & 6 & $\frac{18}{5}$ & 3.6 \\
\hline$\Rightarrow(\overline{\mathbf{0}}, \overline{\mathbf{0}} ; 1,1,1,1) \rightarrow$ & 2 & 1 & $\frac{1}{2}$ & $\frac{7}{3}$ & 2.33333 \\
\hline$\Rightarrow(\overline{\mathbf{0}+\mathbf{0}} ; 1,1,1,1) \Rightarrow$ & 2 & 1 & $\frac{1}{2}$ & $\frac{7}{30}$ & 0.233333 \\
\hline$\Rightarrow(\overline{\mathbf{0}}, \overline{\mathbf{1}} ; 1,1,1) \Rightarrow(\overline{\mathbf{0}}) \Rightarrow \overline{\mathbf{0}} ; 2) \Rightarrow$ & 1 & 1 & 8 & $\frac{56}{15}$ & 3.73333 \\
\hline$\rightarrow(\overline{\mathbf{0}}, \overline{\mathbf{0}}) \Rightarrow(\overline{\mathbf{0}}, \overline{\mathbf{0}} ; 1,1) \rightarrow$ & 1 & 1 & 12 & $\frac{2}{15}$ & 0.133333 \\
\hline$\Rightarrow(\overline{\mathbf{0}}+\mathbf{0}) \Rightarrow(\overline{\mathbf{0}}, \overline{\mathbf{0}} ; 1,1) \Rightarrow$ & 2 & 1 & 6 & $\frac{1}{30}$ & 0.0333333 \\
\hline$\Rightarrow(\overline{\mathbf{0}}, \overline{\mathbf{0}}) \Rightarrow(\overline{\mathbf{0}}+\mathbf{0} ; 1,1) \Rightarrow$ & 2 & 1 & 6 & $\frac{1}{90}$ & 0.0111111 \\
\hline$\Rightarrow(\overline{\mathbf{0}}, \overline{\mathbf{0}}) \Rightarrow(\overline{\mathbf{0}}, \overline{\mathbf{1}} ; 1) \Rightarrow$ & 1 & 1 & 48 & $\frac{4}{45}$ & 0.0888889 \\
\hline$\rightarrow(\overline{\mathbf{0}}, \overline{\mathbf{0}}) \Rightarrow(\overline{\mathbf{0}}, \overline{\mathbf{0}}) \Rightarrow(\overline{\mathbf{0}}, \overline{\mathbf{0}}) \rightarrow$ & 2 & 1 & 12 & $\frac{1}{180}$ & 0.00555556 \\
\hline$\Rightarrow(\overline{\mathbf{0}+\mathbf{0}}) \Rightarrow(\overline{\mathbf{0}}, \overline{\mathbf{0}}) \Rightarrow(\overline{\mathbf{0}}, \overline{\mathbf{0}}) \Rightarrow$ & 2 & 1 & 12 & $\frac{1}{360}$ & 0.00277778 \\
\hline
\end{tabular}

Stratum $\mathcal{H}(1,1,1,1,1,1)$. Configurations of closed geodesics.

\begin{tabular}{|c|c|c|c|c|c|}
\hline Degeneration pattern & $\left|\Gamma_{-}\right|$ & $|\Gamma|$ & $M$ & $c \cdot \zeta(2)$ & $c \cdot \zeta(2)$ approx. \\
\hline$\Rightarrow(\overline{\mathbf{0}}, \overline{\mathbf{0}} ; 1,1,1,1) \Rightarrow$ & 2 & 1 & 15 & $\frac{3150}{377}$ & 8.35544 \\
\hline$\Rightarrow(\overline{\mathbf{0}}, \overline{\mathbf{0}}) \Rightarrow(\overline{\mathbf{0}}, \overline{\mathbf{0}} ; 1,1) \Rightarrow$ & 2 & 1 & 180 & $\frac{90}{377}$ & 0.238727 \\
\hline$\Rightarrow(\overline{\mathbf{0}}, \overline{\mathbf{0}}) \Rightarrow(\overline{\mathbf{0}}, \overline{\mathbf{0}}) \Rightarrow(\overline{\mathbf{0}}, \overline{\mathbf{0}}) \Rightarrow$ & 2 & 3 & 120 & $\frac{5}{754}$ & 0.0066313 \\
\hline
\end{tabular}




\section{B.2. Closed Geodesics for Nonconnected Strata in Genus 4}

Component $\mathcal{H}^{h y p}(6)$.

\begin{tabular}{|c|c|c|c|c|}
\hline Degeneration pattern & $\left|\Gamma_{-}\right|$ & $|\Gamma|$ & $c \cdot \zeta(2)$ & $c \cdot \zeta(2)$ approx. \\
\hline$\rightarrow(\overline{\mathbf{2}}, \overline{\mathbf{2}}) \rightarrow$ & 2 & 1 & $\frac{384}{25}$ & 15.36 \\
\hline$\Rightarrow(\overline{\mathbf{2}+\mathbf{2}}) \Rightarrow$ & 2 & 1 & $\frac{6}{1}$ & 6. \\
\hline$\rightarrow(\overline{\mathbf{0}}, \overline{\mathbf{0}}) \rightarrow(\overline{\mathbf{1}+\mathbf{1}}) \rightarrow$ & 2 & 1 & $\frac{84}{25}$ & 3.36 \\
\hline$\rightarrow(\overline{\mathbf{0}+\mathbf{0}}) \rightarrow(\overline{\mathbf{1}}, \overline{\mathbf{1}}) \rightarrow$ & 2 & 1 & $\frac{896}{225}$ & 3.98222 \\
\hline
\end{tabular}

Component $\mathcal{H}^{\text {even }}(6)$.

\begin{tabular}{|c|c|c|c|c|}
\hline$\rightarrow(\overline{\mathbf{0}}, \overline{\mathbf{4}}) \rightarrow$ & 1 & 1 & $\frac{30375}{4096}$ & 7.41577 \\
\hline$\Rightarrow(\overline{\mathbf{0}+\mathbf{4}}) \Rightarrow$ & 1 & 1 & $\frac{10125}{8192}$ & 1.23596 \\
\hline$\Rightarrow(\overline{\mathbf{1}+\mathbf{3}}) \Rightarrow$ & 1 & 1 & $\frac{875}{256}$ & 3.41797 \\
\hline$\rightarrow(\overline{\mathbf{2}}, \overline{\mathbf{2}}) \rightarrow$ & 2 & 1 & $\frac{405}{128}$ & 3.16406 \\
\hline$\rightarrow(\overline{\mathbf{1}}, \overline{\mathbf{3}}) \rightarrow$ & 1 & 1 & $\frac{15}{1}$ & 15. \\
\hline$\rightarrow(\overline{\mathbf{0}+\mathbf{0}}) \rightarrow(\overline{\mathbf{0}}, \overline{\mathbf{2}}) \rightarrow$ & 1 & 1 & $\frac{2835}{2048}$ & 1.38428 \\
\hline$\rightarrow(\overline{\mathbf{0}}, \overline{\mathbf{0}}) \rightarrow(\overline{\mathbf{0}+\mathbf{2}}) \rightarrow$ & 1 & 1 & $\frac{2835}{4096}$ & 0.692139 \\
\hline$\rightarrow(\overline{\mathbf{0}+\mathbf{0}}) \Rightarrow(\overline{\mathbf{1}+\mathbf{1}}) \rightarrow$ & 1 & 1 & $\frac{2835}{8192}$ & 0.346069 \\
\hline
\end{tabular}

Component $\mathcal{H}^{\text {odd }}(6)$.

\begin{tabular}{|c|c|c|c|c|}
\hline$\rightarrow(\overline{\mathbf{0}}, \overline{\mathbf{4}}) \rightarrow$ & 1 & 1 & $\frac{350}{27}$ & 12.963 \\
\hline$\Rightarrow(\overline{\mathbf{0}+\mathbf{4}}) \Rightarrow$ & 1 & 1 & $\frac{175}{81}$ & 2.16049 \\
\hline$\Rightarrow(\overline{\mathbf{1}+\mathbf{3}}) \Rightarrow$ & 1 & 1 & $\frac{25}{32}$ & 0.78125 \\
\hline$\Rightarrow(\overline{\mathbf{2}+\mathbf{2}}) \Rightarrow$ & 2 & 1 & $\frac{175}{162}$ & 1.08025 \\
\hline$\rightarrow(\overline{\mathbf{2}}, \overline{\mathbf{2}}) \rightarrow$ & 2 & 1 & $\frac{105}{16}$ & 6.5625 \\
\hline$\rightarrow(\overline{\mathbf{1}}, \overline{\mathbf{3}}) \rightarrow$ & 1 & 1 & $\frac{256}{27}$ & 9.48148 \\
\hline$\rightarrow(\overline{\mathbf{0}+\mathbf{0}}) \Rightarrow(\overline{\mathbf{0}+\mathbf{2}}) \rightarrow$ & 1 & 1 & $\frac{7}{32}$ & 0.21875 \\
\hline$\rightarrow(\overline{\mathbf{0}+\mathbf{0}}) \Rightarrow(\overline{\mathbf{2}+\mathbf{0}}) \rightarrow$ & 1 & 1 & $\frac{7}{32}$ & 0.21875 \\
\hline$\rightarrow(\overline{\mathbf{0}+\mathbf{0}}) \rightarrow(\overline{\mathbf{1}}, \overline{\mathbf{1}}) \rightarrow$ & 2 & 1 & $\frac{7}{27}$ & 0.259259 \\
\hline$\rightarrow(\overline{\mathbf{0}+\mathbf{0}}) \rightarrow(\overline{\mathbf{0}+\mathbf{0}}) \rightarrow(\overline{\mathbf{0}}, \overline{\mathbf{0}}) \rightarrow$ & 2 & 1 & $\frac{35}{432}$ & 0.0810185 \\
\hline$\rightarrow(\overline{\mathbf{0 + \mathbf { 0 }}} \rightarrow(\overline{\mathbf{0}+\mathbf{0}}) \Rightarrow(\overline{\mathbf{0}+\mathbf{0}}) \rightarrow$ & 2 & 1 & $\frac{35}{864}$ & 0.0405093 \\
\hline
\end{tabular}


Configurations of closed geodesics.

Component $\mathcal{H}^{h y p}(3,3)$.

\begin{tabular}{|c|c|c|c|c|}
\hline Degeneration pattern & $\left|\Gamma_{-}\right|$ & $|\Gamma|$ & $c \cdot \zeta(2)$ & $c \cdot \zeta(2)$ approx. \\
\hline$\Rightarrow(\overline{\mathbf{2}}, \overline{\mathbf{2}}) \Rightarrow$ & 2 & 1 & $\frac{15}{2}$ & 7.5 \\
\hline$\rightarrow(\overline{\mathbf{0}}, \overline{\mathbf{0}}) \rightarrow(\overline{\mathbf{1}}, \overline{\mathbf{1}}) \rightarrow$ & 2 & 1 & $\frac{35}{9}$ & 3.88889 \\
\hline
\end{tabular}

Component $\mathcal{H}^{\text {nonhyp }}(3,3)$.

\begin{tabular}{|c|c|c|c|c|}
\hline Degeneration pattern & $\left|\Gamma_{-}\right|$ & $|\Gamma|$ & $c \cdot \zeta(2)$ & $c \cdot \zeta(2)$ approx. \\
\hline$\Rightarrow(\overline{\mathbf{2}}, \overline{\mathbf{2}}) \Rightarrow$ & 2 & 1 & $\frac{3699}{1120}$ & 3.30268 \\
\hline$\rightarrow(\overline{\mathbf{0}}, \overline{\mathbf{1}} ; 3) \rightarrow$ & 1 & 1 & $\frac{64}{5}$ & 12.8 \\
\hline$\Rightarrow(\overline{\mathbf{0}+\mathbf{1}} ; 3) \Rightarrow$ & 1 & 1 & $\frac{64}{35}$ & 1.82857 \\
\hline$\rightarrow(\overline{\mathbf{0}+\mathbf{0}}) \Rightarrow(\overline{\mathbf{2}}, \overline{\mathbf{0}}) \rightarrow$ & 1 & 1 & $\frac{27}{80}$ & 0.3375 \\
\hline$\rightarrow(\overline{\mathbf{0}}, \overline{\mathbf{0}}) \rightarrow(\overline{\mathbf{1}}, \overline{\mathbf{1}}) \rightarrow$ & 2 & 1 & $\frac{1}{5}$ & 0.2 \\
\hline$\rightarrow(\overline{\mathbf{0}}, \overline{\mathbf{0}}) \rightarrow(\overline{\mathbf{0}+\mathbf{0}}) \Rightarrow(\overline{\mathbf{0}+\mathbf{0}}) \rightarrow$ & 2 & 1 & $\frac{1}{32}$ & 0.03125 \\
\hline
\end{tabular}

Component $\mathcal{H}^{\text {even }}(4,2)$.

\begin{tabular}{|c|c|c|c|c|}
\hline Degeneration pattern & $\left|\Gamma_{-}\right|$ & $|\Gamma|$ & $c \cdot \zeta(2)$ & $c \cdot \zeta(2)$ approx. \\
\hline$\rightarrow(\overline{\mathbf{0}}, \overline{\mathbf{0}} ; 4) \rightarrow$ & 2 & 1 & $\frac{4}{3}$ & 1.33333 \\
\hline$\Rightarrow(\overline{\mathbf{0 + 0}} ; 4) \Rightarrow$ & 2 & 1 & $\frac{4}{21}$ & 0.190476 \\
\hline$\rightarrow(\overline{\mathbf{0}}, \overline{\mathbf{2}} ; 2) \rightarrow$ & 1 & 1 & $\frac{256}{45}$ & 5.68889 \\
\hline$\Rightarrow(\overline{\mathbf{0 + 2}} ; 2) \Rightarrow$ & 1 & 1 & $\frac{256}{315}$ & 0.812698 \\
\hline$\Rightarrow(\overline{\mathbf{1 + 1}} ; 2) \Rightarrow$ & 2 & 1 & $\frac{8}{9}$ & 0.888889 \\
\hline$\Rightarrow(\overline{\mathbf{1}}, \overline{\mathbf{3}}) \Rightarrow$ & 1 & 1 & $\frac{32768}{8505}$ & 3.85279 \\
\hline$\rightarrow(\overline{\mathbf{1}}, \overline{\mathbf{1}} ; 2) \rightarrow$ & 2 & 1 & $\frac{128}{27}$ & 4.74074 \\
\hline$\rightarrow(\overline{\mathbf{0}+\mathbf{0}}) \rightarrow(\overline{\mathbf{0}}, \overline{\mathbf{0}} ; 2) \rightarrow$ & 2 & 1 & $\frac{8}{27}$ & 0.296296 \\
\hline$\rightarrow(\overline{\mathbf{0}}, \overline{\mathbf{0}}) \rightarrow(\overline{\mathbf{0}+\mathbf{0}} ; 2) \rightarrow$ & 2 & 1 & $\frac{16}{135}$ & 0.118519 \\
\hline$\rightarrow(\overline{\mathbf{0}}, \overline{\mathbf{0}}) \rightarrow(\overline{\mathbf{0}}, \overline{\mathbf{2}}) \rightarrow$ & 1 & 1 & $\frac{32}{45}$ & 0.711111 \\
\hline$\Rightarrow(\overline{\mathbf{0 + 0}}) \Rightarrow(\overline{\mathbf{1 + 1}}) \Rightarrow$ & 2 & 1 & $\frac{2}{45}$ & 0.0444444 \\
\hline$\rightarrow(\overline{\mathbf{0}+\mathbf{0}}) \Rightarrow(\overline{\mathbf{1}}, \overline{\mathbf{1}}) \rightarrow$ & 1 & 1 & $\frac{256}{1215}$ & 0.2107 \\
\hline
\end{tabular}




\begin{tabular}{|c|c|c|c|c|}
\hline Component $\mathcal{H}^{\text {odd }}(4,2)$ & \\
\hline$\rightarrow(\overline{\mathbf{0}}, \overline{\mathbf{0}} ; 4) \rightarrow$ & $\left|\Gamma_{-}\right|$ & $|\Gamma|$ & $c \cdot \zeta(2)$ & $c \cdot \zeta(2)$ approx. \\
\hline$\Rightarrow(\overline{\mathbf{0}+\mathbf{0}} ; 4) \Rightarrow$ & 2 & 1 & $\frac{49}{18}$ & 2.72222 \\
\hline$\rightarrow(\overline{\mathbf{0}}, \overline{\mathbf{2}} ; 2) \rightarrow$ & 2 & 1 & $\frac{7}{18}$ & 0.388889 \\
\hline$\Rightarrow(\overline{\mathbf{0}+\mathbf{2}} ; 2) \Rightarrow$ & 1 & 1 & $\frac{147}{16}$ & 9.1875 \\
\hline$\Rightarrow(\overline{\mathbf{1}+\mathbf{1}} ; 2) \Rightarrow$ & 1 & 1 & $\frac{21}{16}$ & 1.3125 \\
\hline$\Rightarrow(\overline{\mathbf{1}}, \overline{\mathbf{3}}) \Rightarrow$ & 2 & 1 & $\frac{3}{10}$ & 0.3 \\
\hline$\rightarrow(\overline{\mathbf{1}}, \overline{\mathbf{1}} ; 2) \rightarrow$ & 1 & 1 & $\frac{128}{45}$ & 2.84444 \\
\hline$\rightarrow(\overline{\mathbf{0}+\mathbf{0}}) \Rightarrow(\overline{\mathbf{0}+\mathbf{0}} ; 2) \rightarrow$ & 1 & 1 & $\frac{7}{80}$ & 0.0875 \\
\hline$\Rightarrow(\overline{\mathbf{0}+\mathbf{0}}) \Rightarrow(\overline{\mathbf{0}+\mathbf{2}}) \Rightarrow$ & 1 & 1 & $\frac{21}{320}$ & 0.065625 \\
\hline$\rightarrow(\overline{\mathbf{0}+\mathbf{0}}) \Rightarrow(\overline{\mathbf{1}}, \overline{\mathbf{1}}) \rightarrow$ & 1 & 1 & $\frac{7}{45}$ & 0.155556 \\
\hline$\rightarrow(\overline{\mathbf{0 + \mathbf { 0 }}}) \rightarrow(\overline{\mathbf{0}}, \overline{\mathbf{0}}) \rightarrow(\overline{\mathbf{0}}, \overline{\mathbf{0}}) \rightarrow$ & 2 & 1 & $\frac{7}{144}$ & 0.0486111 \\
\hline$\rightarrow(\overline{\mathbf{0 + \mathbf { 0 }}}) \Rightarrow(\overline{\mathbf{0}}) \Rightarrow(\overline{\mathbf{0}+\mathbf{0}}) \rightarrow$ & 2 & 1 & $\frac{7}{576}$ & 0.0121528 \\
\hline
\end{tabular}

\begin{tabular}{|c|c|c|c|c|}
\hline Component & $\mathcal{H}^{\text {even }}(2,2,2)$ \\
\hline$\Rightarrow(\overline{\mathbf{0}}, \overline{\mathbf{0}} ; 2,2) \rightarrow$ & 2 & 1 & $\frac{180}{37}$ & 4.86486 \\
\hline$\Rightarrow(\overline{\mathbf{0}+\mathbf{0}} ; 2,2) \Rightarrow$ & 2 & 1 & $\frac{45}{74}$ & 0.608108 \\
\hline$\rightarrow(\overline{\mathbf{1}}, \overline{\mathbf{1}} ; 2) \Rightarrow$ & 2 & 1 & $\frac{225}{37}$ & 6.08108 \\
\hline$\Rightarrow(\overline{\mathbf{0}}, \overline{\mathbf{0}}) \rightarrow(\overline{\mathbf{0}}, \overline{\mathbf{0}} ; 2) \rightarrow(\overline{\mathbf{1}}, \overline{\mathbf{1}}) \Rightarrow$ & 2 & 1 & $\frac{225}{296}$ & 0.760135 \\
\hline & 2 & 1 & $\frac{5}{37}$ & 0.135135 \\
\hline
\end{tabular}

\begin{tabular}{|c|c|c|c|c|}
\hline \multicolumn{5}{|c|}{ Component $\mathcal{H}^{\text {odd }}(2,2,2)$} \\
\hline$\Rightarrow(\overline{\mathbf{0}}, \overline{\mathbf{0}} ; 2,2) \rightarrow$ & 2 & 1 & $\frac{252}{31}$ & 8.12903 \\
\hline$\Rightarrow(\overline{\mathbf{1}}, \overline{\mathbf{1}} ; 2) \Rightarrow$ & 2 & 1 & $\frac{63}{62}$ & 1.01613 \\
\hline$\Rightarrow(\overline{\mathbf{0}+\mathbf{0}}) \Rightarrow(\overline{\mathbf{0}+\mathbf{0}} ; 2) \Rightarrow$ & 2 & 1 & $\frac{144}{31}$ & 4.64516 \\
\hline$\Rightarrow(\overline{\mathbf{0}+\mathbf{0}}) \Rightarrow(\overline{\mathbf{1}}, \overline{\mathbf{1}}) \Rightarrow$ & 2 & 1 & $\frac{16}{155}$ & 0.0580645 \\
\hline$\rightarrow(\overline{\mathbf{0}}, \overline{\mathbf{0}}) \rightarrow(\overline{\mathbf{0}}, \overline{\mathbf{0}}) \rightarrow(\overline{\mathbf{0}}, \overline{\mathbf{0}}) \rightarrow$ & 2 & 3 & $\frac{4}{93}$ & 0.0430108 \\
\hline$\Rightarrow(\overline{\mathbf{0}+\mathbf{0}}) \Rightarrow(\overline{\mathbf{0}+\mathbf{0}}) \Rightarrow(\overline{\mathbf{0}+\mathbf{0}}) \Rightarrow$ & 2 & 3 & $\frac{1}{186}$ & 0.00537634 \\
\hline
\end{tabular}




\section{REFERENCES}

[At] M. Atiyah, Riemann surfaces and spin structures, Ann. scient. ÉNS $4^{e}$ série 4 (1971), 47-62.

[C] E. Calabi, An intrinsic characterization of harmonic 1-forms, Global Analysis, Papers in Honor of K.Kodaira, (D.C.Spencer and S.Iyanaga, ed.), 1969, pp. 101-117.

[EMa] A. Eskin, H. Masur, Asymptotic formulas on flat surfaces, Ergodic Theory and Dynamical Systems, 21:2 (2001), 443-478.

[EZo] A. Eskin, A. Zorich, Billiards in rectangular polygons, to appear.

[EOk] A. Eskin, A. Okounkov. Asymptotics of number of branched coverings of a torus and volumes of moduli spaces of holomorphic differentials, Inventiones Mathematicae, 145:1 (2001), 59-104.

[G] E. Gutkin. Billiards in polygons. Physica D, 19, (1986), 311-333.

[GJ] E. Gutkin, C. Judge. Affine mappings of translation surfaces: geometry and arithmetic. Duke Math. J., 103 (2000), no. 2, 191-213.

$[\mathrm{HM}]$ J. Hubbard, H. Masur, Quadratic differentials and foliations, Acta Math. 142 (1979), 221-274.

[HuS] P. Hubert, T. A. Schmidt, Invariants of translation surfaces, Ann. Inst. Fourier (Grenoble) $\mathbf{5 1}$ (2001), no. 2, 461-495.

[J] D. Johnson, Spin structures and quadratic forms on surfaces, J. London Math. Soc. (2) 22 (1980), 365-373.

[KaZe] A. Katok, A. Zemlyakov. Topological transitivity of billiards in polygons. Math. Notes, 18, (1975), 760-764.

[KMS] S. Kerckhoff, H. Masur, J. Smillie. Ergodicity of Billiard Flows and Quadratic Differentials. Ann. of Math., 124, (1986), 293-311.

[Ko] M.Kontsevich, Lyapunov exponents and Hodge theory. "The mathematical beauty of physics" (Saclay, 1996), (in Honor of C. Itzykson) 318-332, Adv. Ser. Math. Phys., 24, World Sci. Publishing, River Edge, NJ, 1997.

[KonZo] M. Kontsevich, A. Zorich, Connected components of the moduli spaces of Abelian differentials, Inventiones Mathematicae, 153 (3) (2003), 631-678.

[M] H. Masur, Interval exchange transformations and measured foliations, Ann of Math. 115 (1982) 169-200.

[MS] H. Masur, J. Smillie, Hausdorff dimension of sets of nonergodic foliations, Ann. of Math. 134 (1991) 455-543.

[MT] H. Masur, S. Tabachnikov, Flat structures and rational billiards, Handbook on Dynamical systems, Vol. 1A, 1015-1089, North-Holland, Amsterdam, 2002.

[S] K. Strebel Quadratic differentials. Springer-Verlag, 1984.

[Ve1] W. Veech, Teichmuller geodesic flow, Ann. Math. 124 (1986) 441-530

[Ve2] W. Veech. Moduli spaces of quadratic differentials. J. D'Analyse Math., 55, (1990), 117171.

[Ve3] W. Veech. Teichmuller curves in moduli space. Eisenstein series and an application to triangular billiards. Invent. Math., 97, (1990), 117-171.

[Ve4 ] W. Veech, Siegel measures, Annals of Math. 148 (1998) 895-944

[Zo] A. Zorich, Square tiled surfaces and Teichmüller volumes of the moduli spaces of Abelian differentials, in collection "Rigidity in Dynamics and Geometry", M. Burger, A. Iozzi (Editors), Springer Verlag, 2002, 459-471. 